\title{
Effects of Surface Roughness and Vortex Generators on the LS(1)-0417MOD Airfoil
}

R. L. Reuss

M. J. Hoffman

G. M. Gregorek

The Ohio State University

Columbus, Ohio

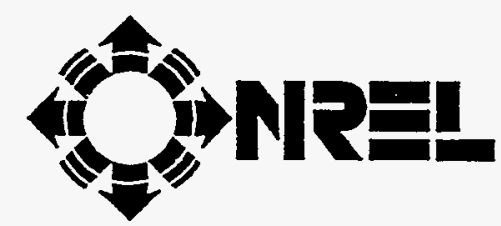

National Renewable Energy Laboratory 1617 Cole Boulevard Golden, Colorado 80401-3393

A national laboratory of the U.S. Department of Energy Managed by Midwest Research Institute for the U.S. Department of Energy under Contract No. DE-AC36-83CH10093 


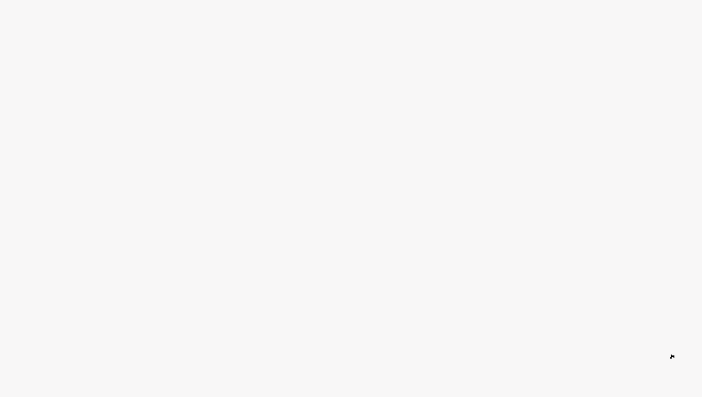

$\cdot$ 


\section{Effects of Surface Roughness and Vortex Generators on the LS(1)-0417MODAirfoil}

\section{R. L. Reuss}

M. J. Hoffman

G. M. Gregorek

The Ohio State University

Columbus, Ohio

NREL Technical Monitor:

C. P. Butterfield

\section{MASTER}

$-$

National Renewable Energy Laboratory 1617 Cole Boulevard

Golden, Colorado 80401-3393

A national laboratory of the U.S. Department of Energy Managed by Midwest Research Institute for the U.S.. Department of Energy under contract No. DE-AC36-83CH10093

Prepared under Subcontract No. XF-1-11009-3

December 1995 


\title{
NOTICE
}

This report was prepared as an account of work sponsored by an agency of the United States government. Neither the United States government nor any agency thereof, nor any of their employees, makes any warranty, express or implied, or assumes any legal liability or responsibility for the accuracy, completeness, or usefulness of any information, apparatus, product, or process disclosed, or represents that its use would not infringe privately owned rights. Reference herein to any specific commercial product, process, or service by trade name, trademark, manufacturer, or otherwise does not necessarily constitute or imply its endorsement, recommendation, or favoring by the United States govemment or any agency thereof. The views and opinions of authors expressed herein do not necessarily state or reflect those of the United States

\begin{abstract}
Wind turbines in the field can be subjected to many and varying wind conditions, including high winds with the rotor locked or with yaw excursions. In some cases, the rotor blades may be subjected to unusually large angles of attack that possibly result in unexpected loads and deflections. To better understand loadings at unusual angles of attack, a wind tunnel test was performed.
\end{abstract}

An 18-inch constant-chord model of the LS(1)-0417MOD airfoil section was tested under two dimensional steady state conditions in the Ohio State University Aeronautical and Astronautical Research Laboratory $7 \times 10$ Subsonic Wind Tunnel. The objective of these tests was to document section lift and moment characteristics under various model and air flow conditions. Surface pressure data was acquired at $-60^{\circ}$ through $+230^{\circ}$ geometric angles of attack, at a nominal 1 million Reynolds number. Cases with and without leading edge grit roughness were investigated. The leading edge roughness was used to simulate hlade conditions encountered on wind turhines in the field Additionally surface nreccure data were 
List of Symbols $\ldots \ldots \ldots \ldots \ldots \ldots \ldots \ldots \ldots \ldots \ldots \ldots \ldots \ldots \ldots \ldots$

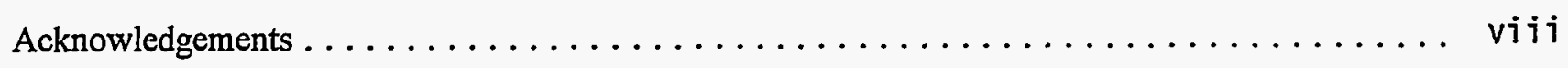

Introduction $\ldots \ldots \ldots \ldots \ldots \ldots \ldots \ldots \ldots \ldots \ldots \ldots \ldots \ldots \ldots \ldots \ldots \ldots$

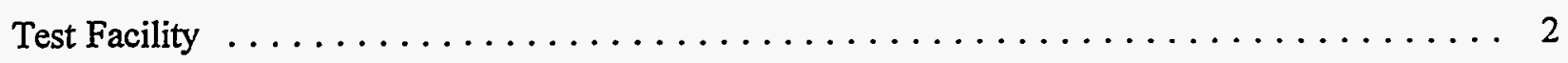

Model Details $\ldots \ldots \ldots \ldots \ldots \ldots \ldots \ldots \ldots \ldots \ldots \ldots \ldots \ldots \ldots \ldots \ldots$

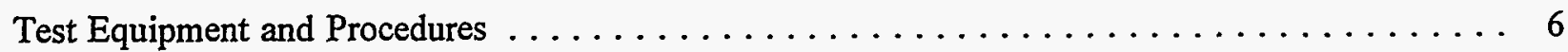

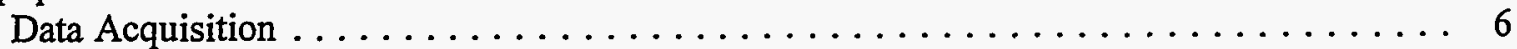

Data Reduction ............................ 7

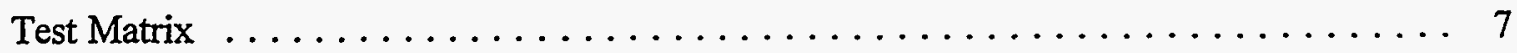

Results and Discussion $\ldots \ldots \ldots \ldots \ldots \ldots \ldots \ldots \ldots \ldots \ldots \ldots \ldots \ldots \ldots$

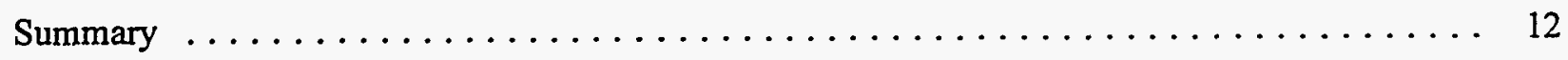

Appendix A: Model and Surface Pressure Tap Coordinates $\ldots \ldots \ldots \ldots \ldots \ldots \ldots \ldots$

Appendix B: Integrated Coefficients and Pressure Distributions $\ldots \ldots \ldots \ldots \ldots \ldots \ldots \ldots$ 


\section{List of Figures}

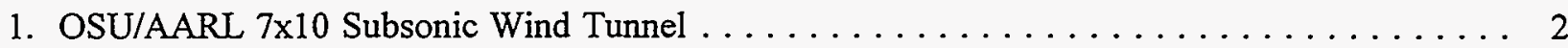

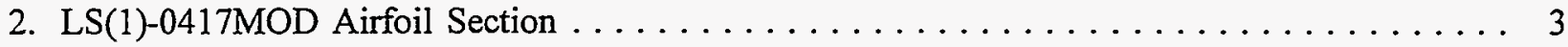

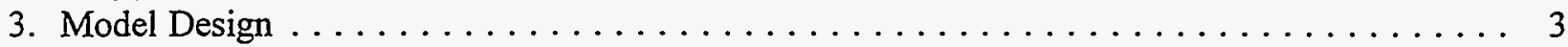

4. Roughness Pattern . . . . . . . . . . . . . . . . . . . . . . . . . 4

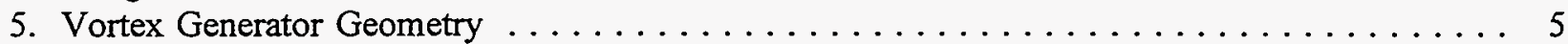

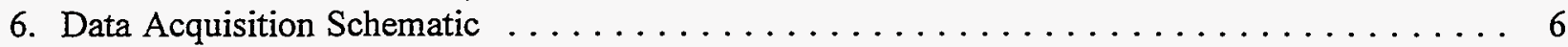

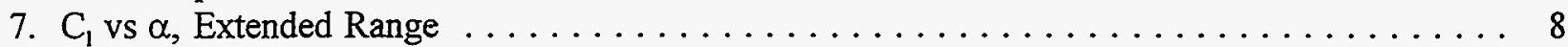

8. $C_{m^{1 / 4}}$ vs $\alpha$, Extended Range $\ldots \ldots \ldots \ldots \ldots \ldots \ldots \ldots \ldots \ldots \ldots \ldots \ldots \ldots \ldots \ldots$

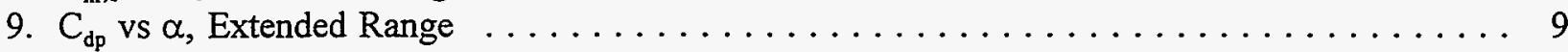

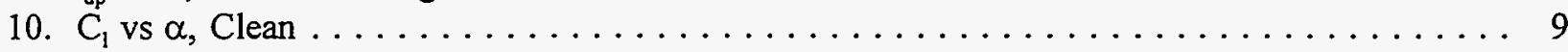

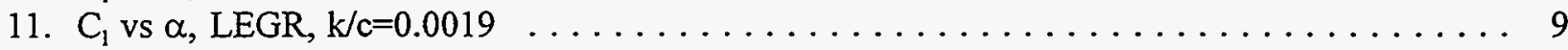

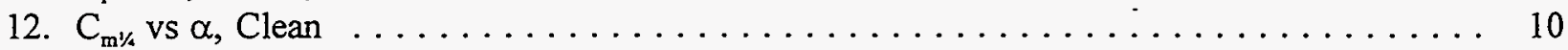

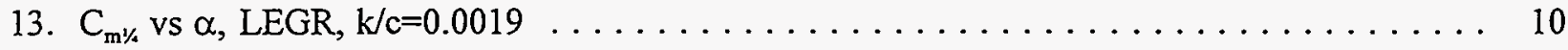

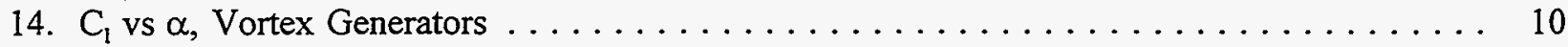

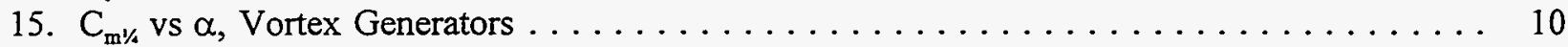

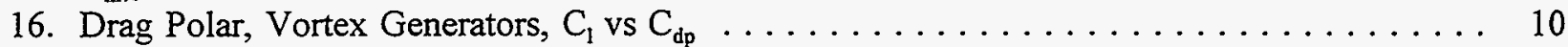

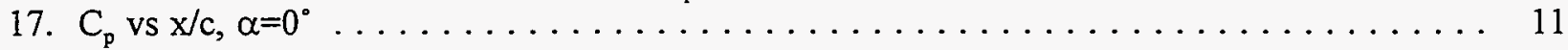

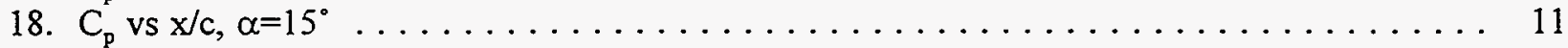

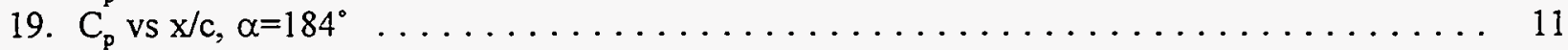

List of Tables $\quad$ Page

1. LS(1)-0417MOD Aerodynamic Parameters Summary $\ldots \ldots \ldots \ldots \ldots \ldots \ldots \ldots \ldots$ 


\section{List of Symbols}

AOA Angle of Attack, degrees

$\alpha \quad$ Angle of Attack, degrees

c Chord Length, inches

$\mathrm{C}_{\mathrm{dmin}} \quad$ Minimum Drag Coefficient

$\mathrm{C}_{\mathrm{dp}} \quad$ Section Pressure (Form) Drag Coefficient

$\mathrm{C}_{\mathrm{dw}} \quad$ Section Drag Coefficient, calculated from Wake momentum deficit

$C_{1} \quad$ Section Lift Coefficient

$\mathrm{C}_{\mathrm{Imax}}$

Section Maximum Lift Coefficient

$C_{m}$

Section Pitching Moment Coefficient

$\mathrm{C}_{\mathrm{mo}}$

Section Pitching Moment Coefficient at zero degrees angle of attack

$\mathrm{C}_{\mathrm{m} / 4} \quad$ Section Pitching Moment Coefficient about the quarter chord

$\mathrm{C}_{\mathrm{p}} \quad$ Pressure Coefficient

$\mathrm{C}_{\mathrm{pmin}} \quad$ Minimum Pressure Coefficient

k

Roughness element height, inches

psi Units of pressure, pounds per square inch

q Dynamic pressure, psi

Re Reynolds number

$\mathrm{x}$

Axis parallel to airfoil reference line, Coordinate in inches

Axis perpendicular to airfoil reference line, Coordinate in inches 


\section{Acknowledgements}

This work was made possible by the efforts and financial support of the National Renewable Energy Laboratory which provided major funding and technical monitoring; the U.S. Department of Energy, which is credited for its funding of this document through the National Renewable Energy Laboratory under contract number DE-AC36-83CH10093 and U.S. Windpower Incorporated which provided funding for models and provided technical assistance. The staff of the Ohio State University Aeronautical and Astronautical Research Laboratory appreciate the contributions made by personnel from both organizations. 


\section{Introduction}

Wind turbines in the field can be subjected to many and varying wind conditions, including high winds with the rotor locked or with yaw excursions. In some cases the rotor blades may be subjected to unusually large angles of attack that possibly result in unexpected loads and deflections. To better understand loadings at unusual angles of attack, a wind tunnel test was performed. An 18-inch constantchord model of the LS(1)-0417MOD airfoil section was tested under two-dimensional, steady state conditions in the Ohio State University Aeronautical and Astronautical Research Laboratory (OSU/AARL) $7 \times 10$ Subsonic Wind Tunnel $(7 \times 10)$. The objective of these tests was to document section lift and moment characteristics under various model and air flow conditions. These included a normal angle of attack range of $-20^{\circ}$ to $+40^{\circ}$, an extended angle of attack range of $-60^{\circ}$ to $+230^{\circ}$, applications of leading edge grit roughness (LEGR), and use of vortex generators (VGs), all at chord Reynolds numbers as high as possible for the particular model configuration. To realistically satisfy these conditions the $7 \times 10$ offered a tunnel-height-to-model-chord ratio of 6.7, suggesting low interference effects even at the relatively high lift and drag conditions expected during the test. Significantly, it also provided chord Reynolds numbers up to 2.0 million.

Knowing the LS(1)-0417MOD model would later be run in the OSU/AARL 3x5 Subsonic Wind Tunnel ( $3 \times 5)$, the present test setup and methods were kept as similar as possible to those for the $3 \times 5$. This will allow a direct comparison of data obtained in the two wind tunnels. Consequently, most of the data acquisition equipment was moved from the $3 \times 5$ to the $7 \times 10$. Minor changes were made to the system in order to adapt the equipment to the larger facility. Also, so that the LS(1)-0417MOD model could be used in both tunnels, it was specially designed to include a central 3-foot span sensing section with removable, contoured, spanwise extensions.

A "standard" grit pattern was applied in all LEGR cases. The grit pattern was developed by U.S. Windpower, OSU/AARL, and the University of Texas, Permian Basin. The VGs were provided to OSU/AARL by U.S. Windpower. Detailed discussion of the grit pattern and VGs can be found in the Section, Model Details.

Reynolds numbers of $1,1.5$, and 2 million were tested for normal angle of attack range cases $\left(-20^{\circ}\right.$ to $+40^{\circ}$ ). At 1 million Reynolds number, the model was additionally swept through the extended angle of attack range. The model buffeted at higher dynamic pressures, thus precluding higher Reynolds number data for extended angle of attack range. However, both clean and LEGR data were taken for all useable tunnel conditions. Finally, VG effects were evaluated over the normal angle of attack range, for Reynolds numbers of 1 and 1.5 million, and for clean and LEGR cases. The VGs were tested at the $30 \%$ chord upper surface station only; any attempt at higher Reynolds numbers with VGs consistently result in VGs separating from the model. Scheduling constraints precluded any significant effort to alleviate the VG attachment problem. 


\section{Test Facility}

Tests described here were performed in the OSU/AARL 7x10 subsonic wind tunnel. A schematic of the tunnel is shown in figure 1. There are two test sections in this tunnel: a 7-foot by 10-foot section in which these tests were conducted, and a 16-foot by 14-foot section in which very low speed and high angle of attack testing is performed with large models. The wind tunnel is a closed-circuit, single-return, continuous-flow system. A velocity range of 35 to 180 knots is developed in the $7 \times 10$ test section by a six-blade, fixed-pitch, 20 -foot diameter fan directly driven by a 2000 -horsepower, variable-speed motor. The tunnel's steel outer shell is water spray cooled to control internal air temperature. Its test section floor contains a rotating table which allows adjustment of the model angle of attack through a $290^{\circ}$ range about a vertical axis. A large, long traverse, wake survey probe was not available; consequently, none was installed in the test section.

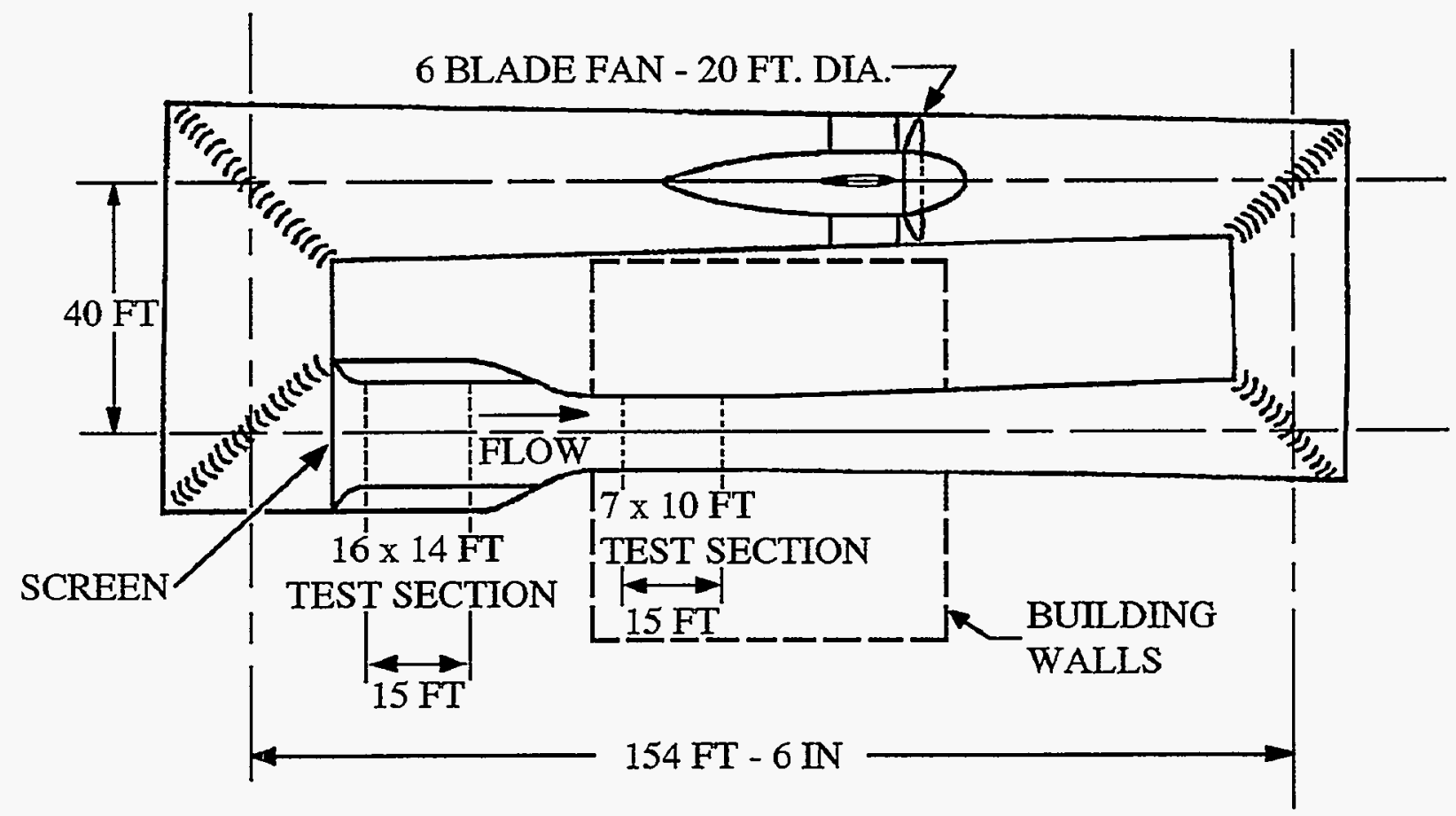

Figure 1. OSU/AARL $7 \times 10$ Subsonic Wind Tunnel 


\section{Model Details}

An 18-inch constant-chord LS(1)-0417MOD airfoil model was designed by OSU/AARL personnel and manufactured by others. Figure 2 shows the airfoil section; the section's measured coordinates are given in Appendix A. The model was made of a carbon composite skin over a foam core. The main load bearing member is a $1 \frac{1}{2}$-inch diameter steel tube that passes through the foam core at the airfoil quarter chord station. Steel and composite ribs and end plates transfer loads from the composite skin to the steel tube. The final surface was hand worked using templates to attain given coordinates within a tolerance of \pm 0.01 inches.

$$
\text { LS(1)-0417MOD }
$$

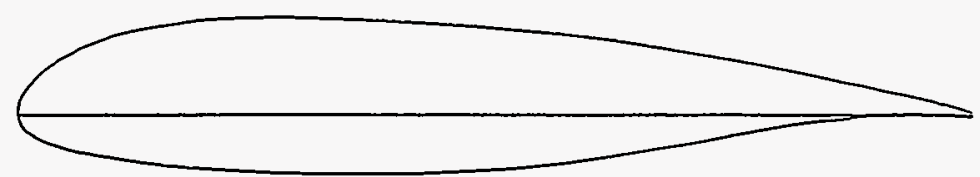

Figure 2. LS(1)-0417MOD Airfoil Section

Since the model also had to be used in the $3 \times 5$ subsonic wind tunnel for additional tests, it was designed with a 3-foot span main sensing section and 2-foot extension panels for each end, shown in figure 3. The extensions, used for $7 \times 10$ tunnel testing, were fabricated with the same contour as the main section. They slide over the steel tube and fasten to the endplates of the main section. Other minor model features were included, such as an extension to the model support tube and an adaptation of the support tube end to accommodate the different angle of attack potentiometer mountings in each facility.

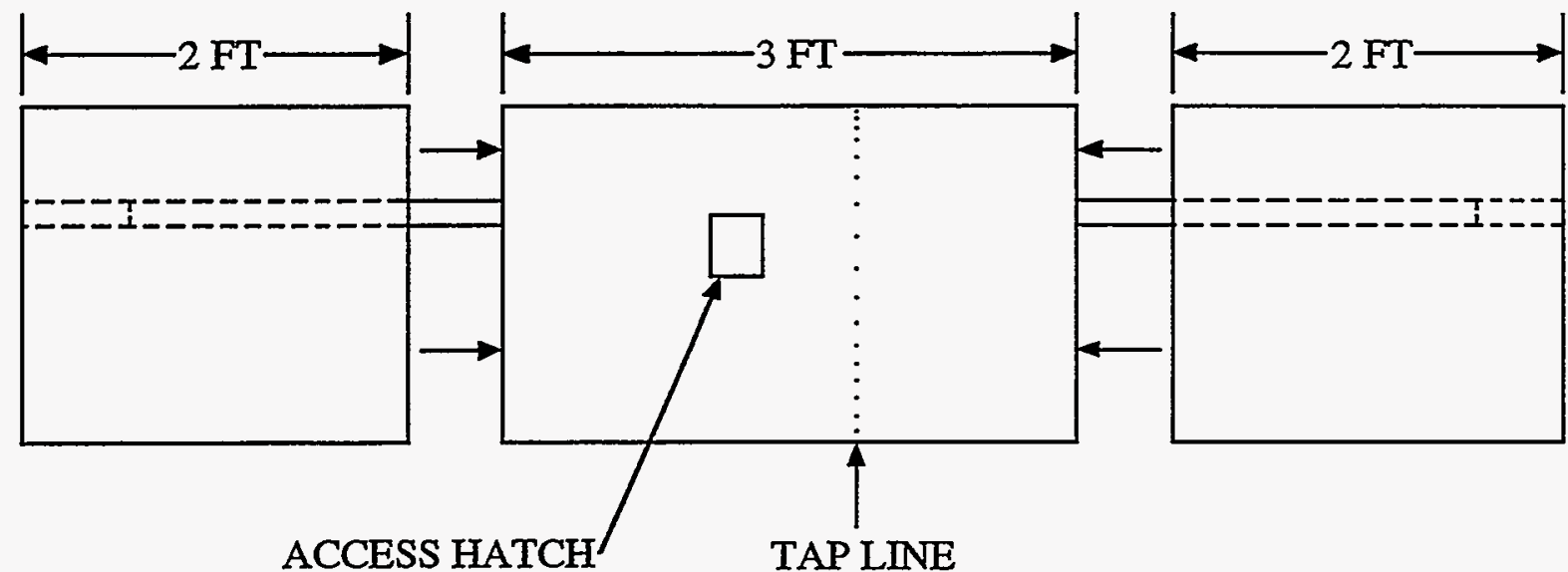

Figure 3. Model Design 
To minimize pressure response times, the lengths of surface pressure tap leadout lines were made as short as possible. Although response time was not particularly important for the present test, it was important for the unsteady testing to be done later in the $3 \times 5$ wind tunnel. Therefore, a compartment was built into the model to hold the pressure scanning modules. This compartment can be accessed through a panel door fitted flush with the model contour on the lower (pressure) surface.

For test cases involving roughness, to have a standard, repeatable pattern with grit as roughness elements was desired. Prior to these tests, grit was lightly blown into a thin layer of spray adhesive or a tape adhesive to obtain a roughened surface on models. A different method was developed and used here. OSU/AARL and U.S. Windpower personnel jointly developed a roughness pattern using a molded insect pattern taken from a wind turbine in the field by personnel at the University of Texas, Permian Basin.

The resultant particle density was 32 particles per square inch in the middle of the pattern, and thinning to 8 particles per square inch at the edge of the pattern. Figure 4 shows the pattern template produced by U.S. Windpower from these specifications. The pattern was repeatedly cut into a steel sheet 4-inches wide and 3-feet long with holes just large enough for one piece of grit. Based on average particle size from the field specimen, standard \#40 lapidary grit was chosen for the roughness elements, giving $\mathrm{k} / \mathrm{c}=0.0019$ for an 18 inch chord model.

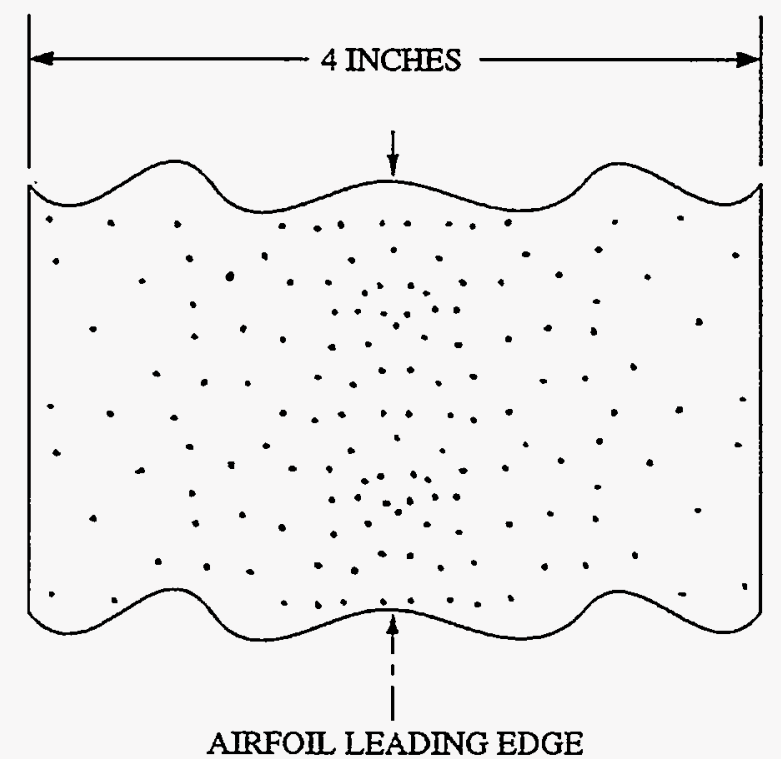

Figure 4. Roughness Pattern

To use the template, 4-inch wide double-tack tape was stuck to one side of the template and grit was poured and brushed from the opposite side. The tape was then removed from the template and transferred to the model. This scheme allowed the same roughness pattern to be replicated for any test.

VGs were applied to the model for some data points. U.S. Windpower provided the VGs with the geometry shown in figure 5. The VGs were pairs of right isosceles triangular shapes set on their longest sides at $30^{\circ}$ included angle to each other and $15^{\circ}$ to the chord line. The pairs were repeated every 1.61 inches in the spanwise direction. This VG configuration was fabricated in 1.53-inch wide injection-molded plastic strips with a 0.036 -inch base-plate thickness. For ease of installation and to minimize damage to the model surface, these strips were fastened at the $30 \%$ chord upper surface station 
using rubber cement between the VG base-plate and model, and thin tape (0.003-inch thick) over the base plate leading and trailing edges.

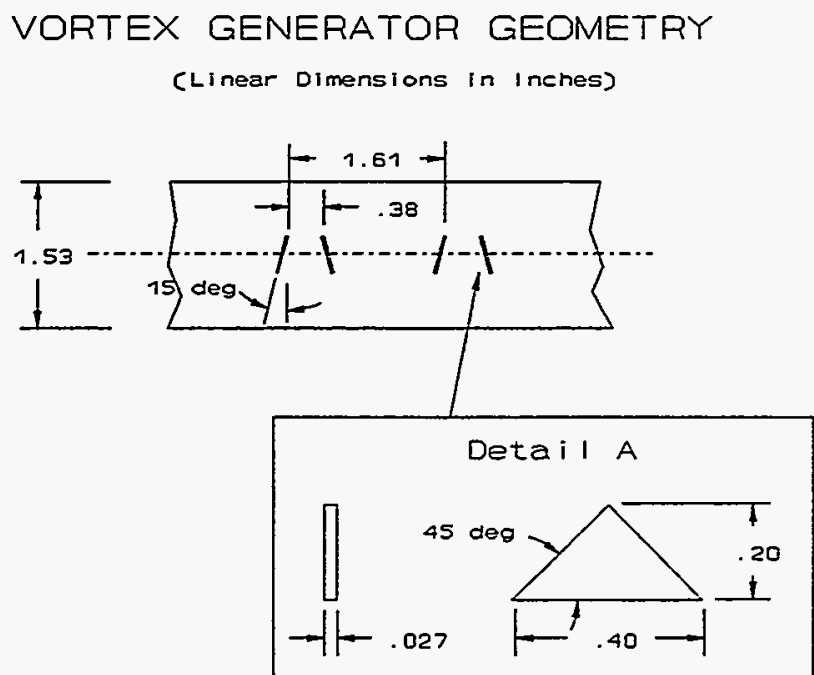

Figure 5. Vortex Generator Geometry 


\section{Test Equipment and Procedures}

Data Acquisition

Data was acquired and processed from up to 60 surface pressure taps, three individual tunnel pressure transducers, and an angle of attack potentiometer. The data acquisition system included an IBM PC compatible 80386-based computer connected to a Pressure Systems Incorporated (PSI) data scanning system. The PSI system included a 780B Data Acquisition and Control Unit (DACU), 780B Pressure Calibration Unit (PCU), 81-IFC scanning module interface, two ESP-32 5-psid range pressure scanning modules (ESPs), and a 30-channel Remotely Addressed Millivolt Module (RAMM-30). Figure 6 shows the data acquisition system schematic.

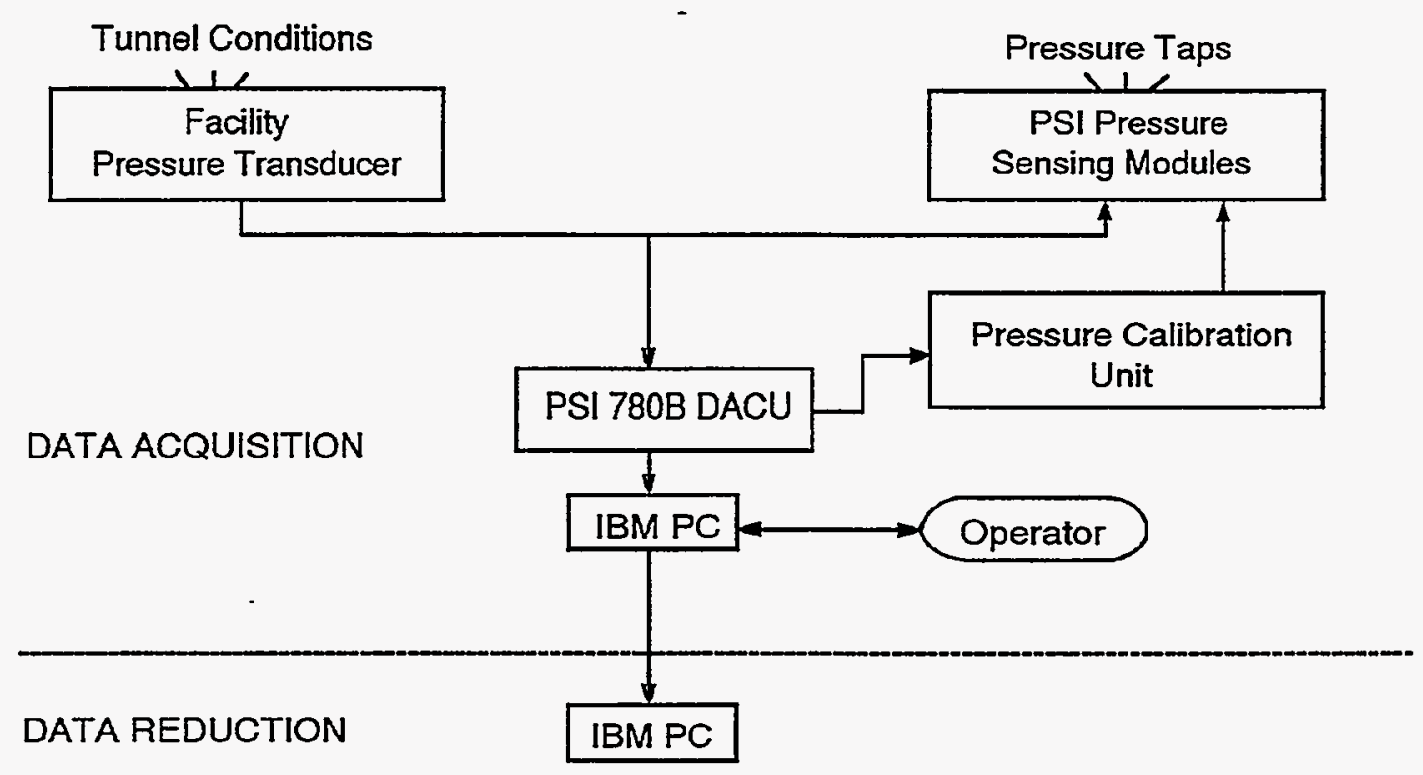

Figure 6. Data Acquisition Schematic

Three individual pressure transducers read tunnel total pressure, tunnel east static pressure, and tunnel west static pressure. Before the test began, these transducers were bench calibrated using a water manometer to determine their sensitivities and offsets. Related values were entered into the data acquisition and reduction program so the transducers could be shunt-resistor calibrated before each series of wind tunnel runs.

The angle of attack potentiometer was a linear rotary potentiometer and was regularly calibrated during the tunnel pressure transducers shunt calibration. The angle of attack calibration was accomplished by taking voltage readings at known values of set angle of attack. This calibration method gave angle of attack readings within $\pm 0.25^{\circ}$ of actual over the entire angle range.

Two ESPs were calibrated simultaneously using the DACU and PCU. At operator request, the DACU commanded the PCU to apply known regulated pressures to the ESPs and read the output voltages from each integrated pressure sensor. From these values, the DACU calculated the calibration coefficients and stored them internally until the coefficients were requested by the controlling computer. This calibration 
was done several times during a run set because the ESPs were installed inside the model and their outputs tended to drift with temperature changes during a test sequence. Frequent online calibrations minimized the effect.

Finally, at operator request, pressure measurements from the airfoil surface taps and all other channels of information were acquired and stored by the DACU and subsequently passed to the controlling computer for final processing.

\section{Data Reduction}

The data reduction routine was incorporated as a section of the data acquisition program. This combined data acquisition and reduction routines allowed data to be reduced online during a test. By quickly reducing selected runs, integrity checks could be made to insure the equipment was working properly and to enable timely decisions about the test matrix.

The ambient pressure and tunnel air temperature were manually input into the computer and were updated regularly. These values, as well as the measurements from the tunnel pressure transducers, were used to calculate tunnel airspeed. As a continuous check of readings, both the tunnel individual pressure transducers and the ESPs read the tunnel total and static pressures.

A typical data point was derived by acquiring twenty data scans of all channels over a 1-second window at each angle of attack and tunnel condition. The reduction portion of the program processed each data scan to coefficient forms $C_{p}, C_{l}, C_{m / /}$, and $C_{d p}$ using the measured surface pressure voltages, calibration coefficients, tap locations and wind tunnel conditions. All scan sets for a given condition were then ensemble averaged to provide one set. All data were saved in electronic form. The data were not corrected for any tunnel wall effects, etc.

\section{Test Matrix}

The test was designed to allow an extended angle of attack range of $-60^{\circ}$ to $230^{\circ}$ and Reynolds numbers of $1,1.5$, and 2 million with and without LEGR. The tabular data in Appendix B contains the actual Reynolds number for each angle of attack. The angle of attack increment was 4-degrees when $\alpha<-20^{\circ}$ or $\alpha>40^{\circ}$, 2-degrees when $-20^{\circ}<\alpha<10^{\circ}$ or $20^{\circ}<\alpha<40^{\circ}$, and 1-degree when $10^{\circ}<\alpha<20^{\circ}$. All test speeds and angles of attack were set for model clean and LEGR conditions.

For some cases, VGs were mounted at the $30 \%$ chord position on the model's upper surface only. The VG strips were provided by U.S. Windpower and were the exact type used on wind turbines in the field. Test conditions while the VGs were applied included clean and LEGR data at 1 and 1.5 million Reynolds numbers over an angle of attack range of $-20^{\circ}$ to $40^{\circ}$.

Unexpected complications during testing forced adjustments to this desired test matrix. Those complications and their effects are elaborated in the next section, Results and Discussion. 


\section{Results and Discussion}

The LS(1)-0417MOD airfoil model was tested at three Reynolds numbers in the 7x10. Unfortunately, due to less than expected model rigidity, the model flexed and fluttered when near perpendicular to the flow at the higher test airspeeds. The tunnel airspeed was reduced for those conditions to reduce dynamic effects and to preserve the model's structural integrity. Consequently, the Reynolds number was not constant for the entire extended angle of attack sweeps and only the nominal 1 million Reynolds number condition was obtained. The Reynolds number was as low as 0.6 million during the nominal 1 million Reynolds number extended angle of attack cases. Also, no wake survey probe was available for the test; only pressure drag from surface pressure integrations is presented.

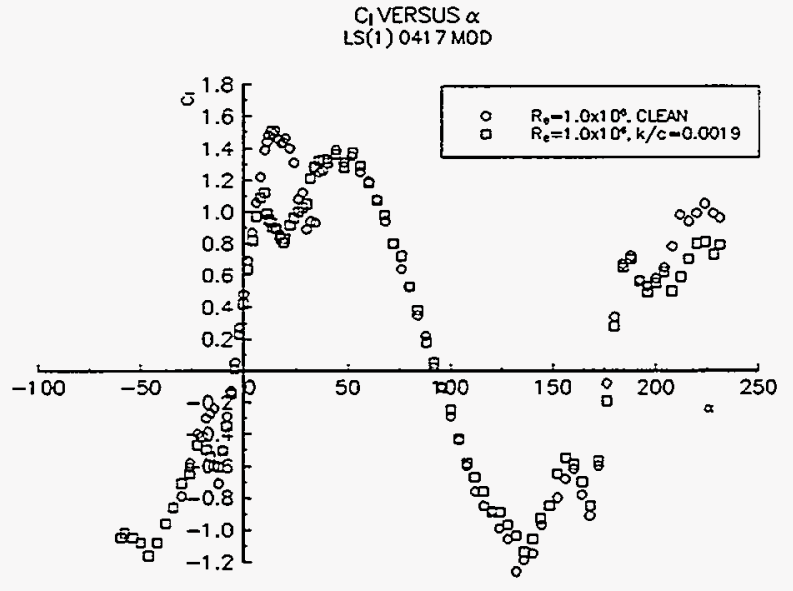

Figure 7. $C_{1}$ vs $\alpha$, Extended Range

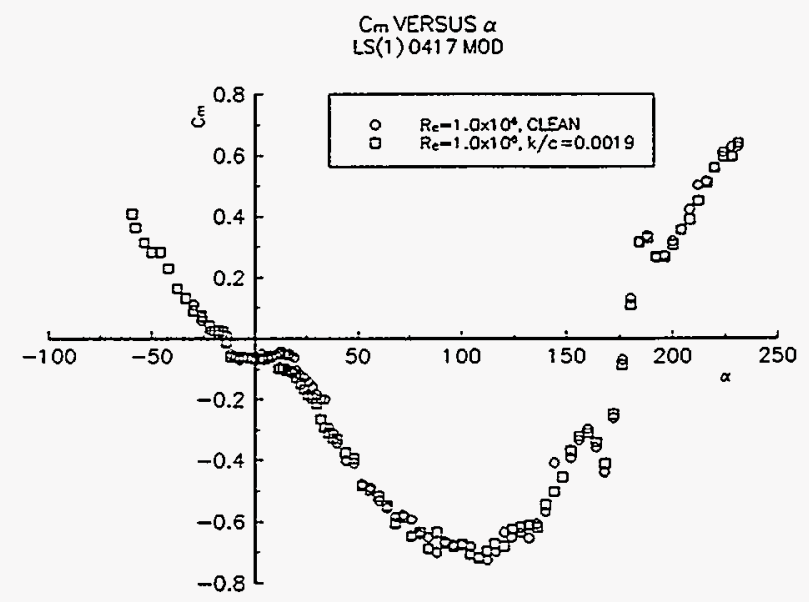

Figure 8. $\mathrm{C}_{\mathrm{m} / \mathrm{s}}$ vs $\alpha$, Extended Range

Figure 7 shows the lift coefficient versus angle of attack for the extended angle of attack sweeps, for the model clean and with LEGR at 1 million Reynolds Number. Increases in lift coefficient occurred when the model was in its post stall region for both positive and negative angles of attack; the maximum lift coefficient occurred just before positive stall and is 1.51. Correspondingly, for the LEGR data, the maximum lift coefficient prior to stall is 1.12 and occurs at a slightly lower angle of attack in comparison with the clean case. The overall maximum lift of the LEGR case does not occur before stall but beyond it near $45^{\circ}$ angle of attack. This can be observed in figure 7. Similar magnitudes of $C_{1}=1.35$ also are apparent in the large angle of attack clean cases.

The quarter chord pitching moment results are shown in figure 8 for the Reynolds number of 1 million. The pitching moment is most negative when the airfoil is at high angles of attack, near $110^{\circ}$. This observation is consistent for both clean and LEGR cases. The pressure drag is shown in figure 9. The highest pressure drag occurs when the model is near $90^{\circ}$ angle of attack. There is some scatter in the data at such conditions, caused by the severely detached, unstable flow on the leeward side of the model.

A number of test runs were made for nominal angles of attack from $-20^{\circ}$ to $+40^{\circ}$. For some of the cases, there is no data shown for the highest angles of attack; the data was discounted as unreliable because the model was buffeting. Figure 10 and 11 show lift coefficient for all the test Reynolds numbers, for the model clean and with LEGR. The maximum positive lift coefficient for the clean cases is about 1.63 and the LEGR data have a $C_{\operatorname{lmax}}$ about 1.2. For the clean cases, the airfoil stalls more abruptly with higher 
Reynolds numbers. Also note that for the 1 million Reynolds number case, the model with LEGR seems to stall more abruptly than the clean airfoil. The average lift curve slope for these data is about 0.1 .

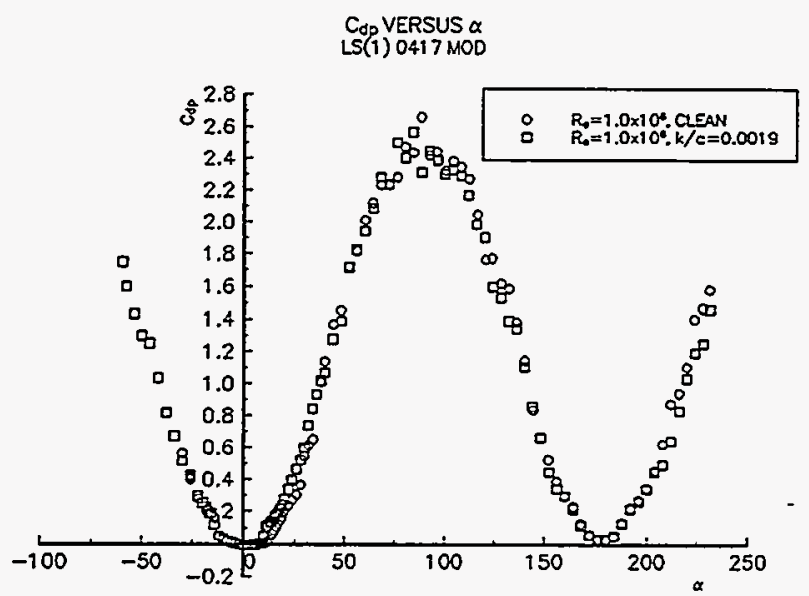

Figure 9. $\mathrm{C}_{\mathrm{dp}}$ vs $\alpha$, Extended Range

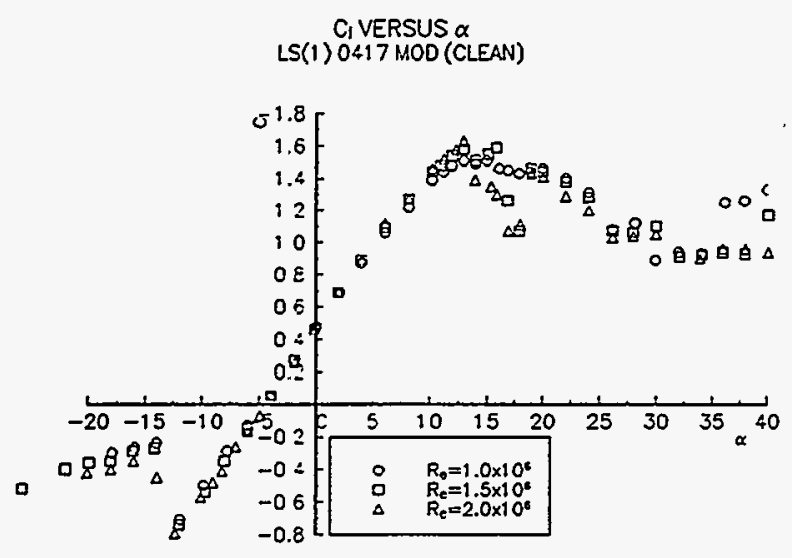

Figure 10. $C_{1}$ vs $\alpha$, Clean

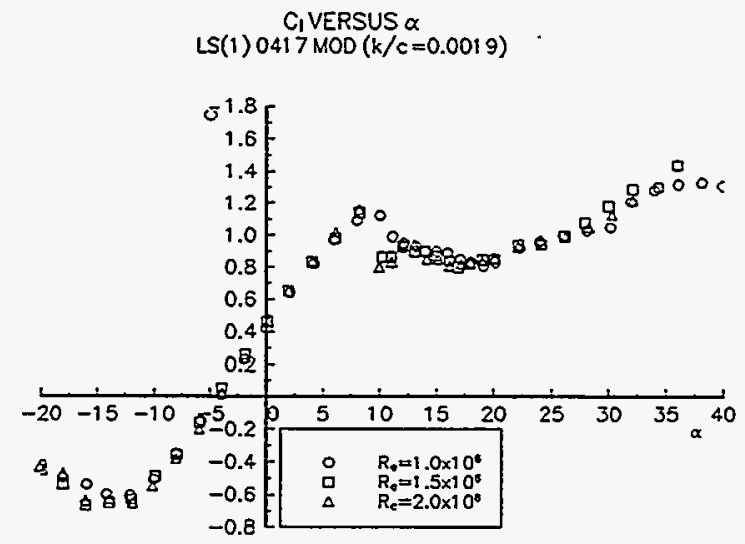

Figure 11. $C_{l}$ vs $\alpha, L E G R, k / c=0.0019$

Figure 12 shows the pitching moment about the quarter chord for the clean cases; figure 13 shows the LEGR cases. The LEGR data show a more positive pitching moment near $0^{\circ}$ angle of attack. However, beyond stall, the pitching moment magnitude increases faster for the model with LEGR than for the clean model. The $\mathrm{C}_{\mathrm{mo}}$ about the quarter chord for the clean case is -0.080 and -0.067 for the LEGR case.

VGs were fitted to the model at the $30 \%$ chord location, upper surface (suction side) only. The results from these cases are shown in figure 14 for lift coefficient, and in figure 15, for pitching moment coefficient. The maximum lift coefficient for the clean case with VGs is near 1.88 and near 1.41 for the LEGR case. This is a $25 \%$ reduction in maximum lift when the airfoil has leading edge roughness. The stall of this airfoil is more abrupt when the VGs are applied, and occurs at a slightly higher angle of attack than without VGs. The pitching moment shows slightly different characteristics with the VGs than without. Pre-stall pitching moment magnitude slightly increases with increased angle of attack with VGs applied, but slightly decreases without VGs. The pitching moment is affected more near stall without VGs than when the VGs are applied. Pressure drag for the VGs cases but is included only for completeness sake in figure 16. This form of drag coefficient is inherently inaccurate because it does not include 
friction drag and should only be used for comparisons within the present data sets.

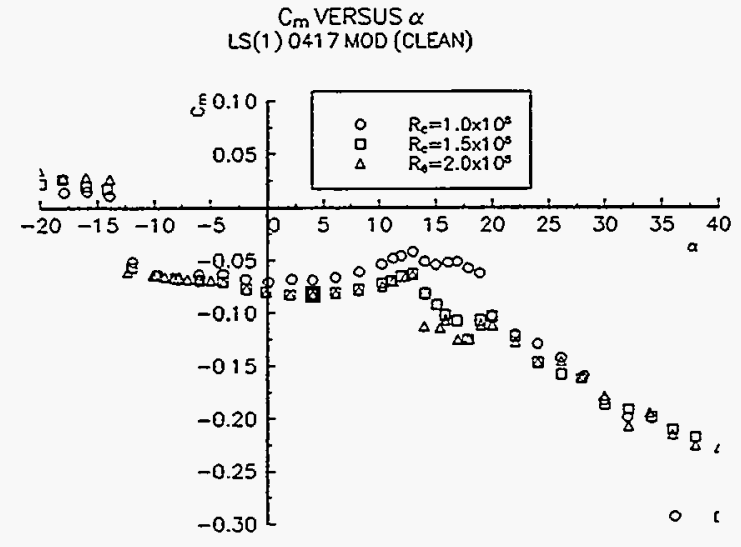

Figure 12. $\mathrm{C}_{\mathrm{m}^{1 / 4}}$ vs $\alpha$, Clean

C, VERSUS a

LS(1) 0417 MOD (VORTEX GENERATORS 307)

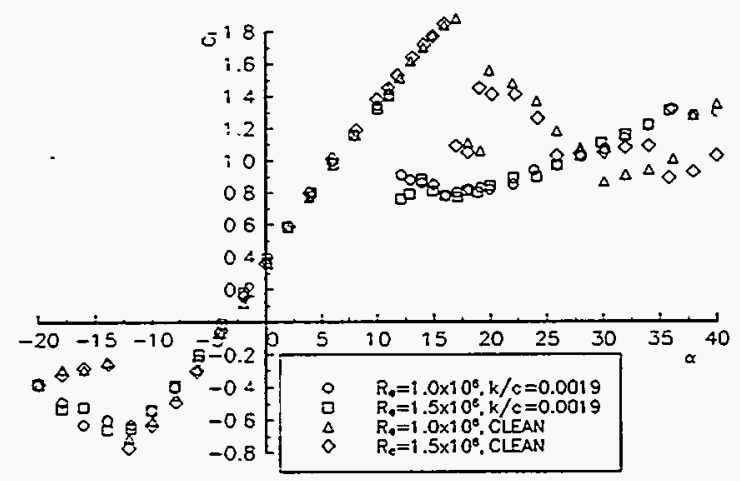

Figure 14. $C_{1}$ vs $\alpha$, Vortex Generators

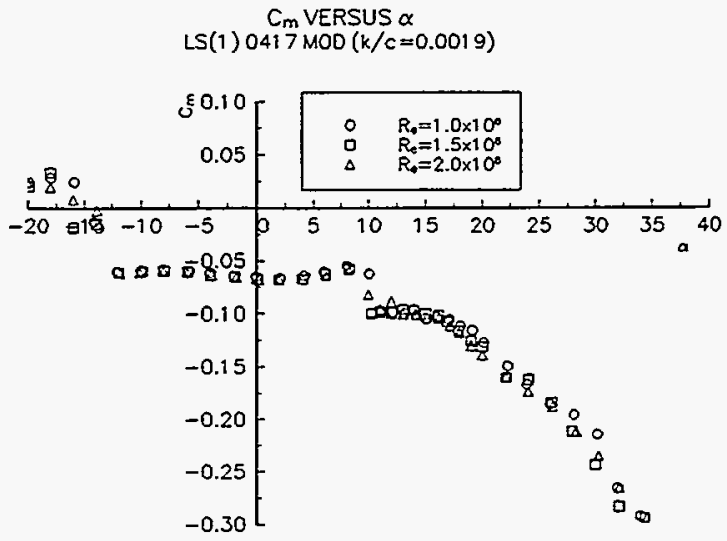

Figure 13. $\mathrm{C}_{\mathrm{m} / \mathrm{s}}$ vs $\alpha, \mathrm{LEGR}, \mathrm{k} / \mathrm{c}=0.0019$

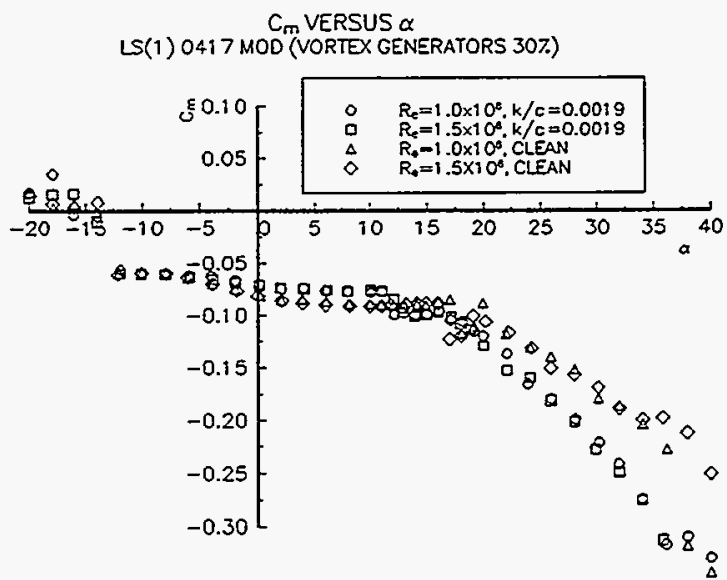

Figure 15. $\mathrm{C}_{\mathrm{m}^{1 / 4}}$ vs $\alpha$, Vortex Generators

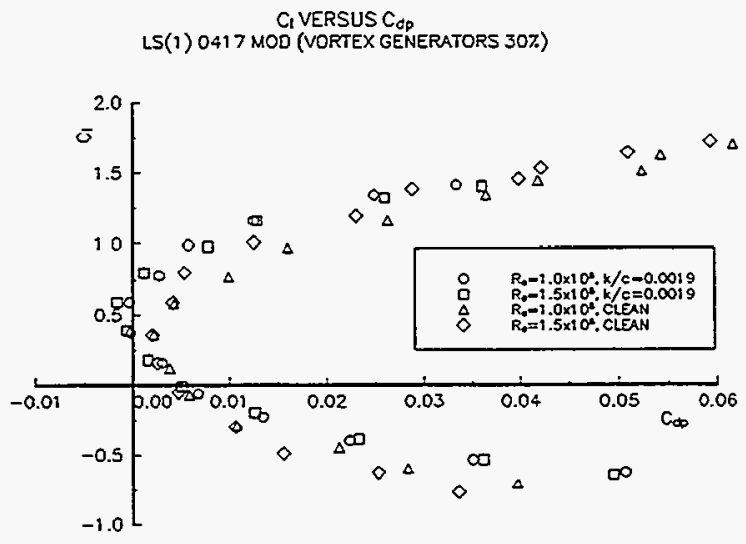

Figure 16. Drag Polar, Vortex Generators, $C_{1}$ vs $C_{d p}$

All the pressure distributions shown are for a Reynolds number of 1 million and show cases of clean, 
LEGR, and VGs with and without LEGR. Figure 17 shows the pressure distributions for $0^{\circ}$ angle of attack. A trend toward reduced pressure magnitudes with LEGR can be observed. At angles of attack near stall, the trending is more apparent. For example, figure 18 shows pressure distributions for a $15^{\circ}$ angle of attack. The data show the model has upper surface flow separation near the $20 \%$ chord station for the LEGR case. It further shows that the VGs increase the pressure magnitudes for both the clean and LEGR cases. Also, the VGs cause the separation point to move aft.

Figure 19 shows pressure data from the model at an angle of attack of $184^{\circ}$ including clean and LEGR pressure distributions. The distributions are almost identical over the airfoil. This shows the roughness does not affect the airflow since it is located, for this unusual case, downstream of the trailing edge.

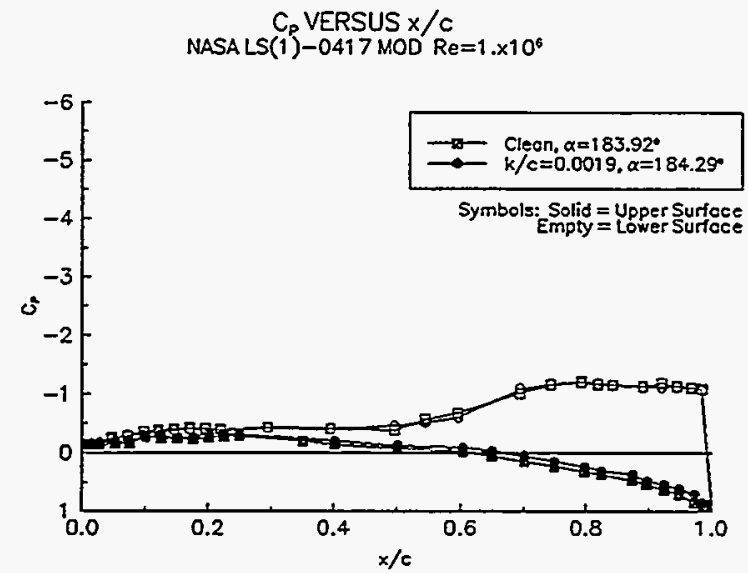

Figure 19. $C_{p} v s x / c, \alpha=184^{\circ}$

The final tests conducted on this wind tunnel model in the $7 \times 10$ were to repeat clean conditions at Reynolds numbers of 1 million and 1.5 million for a smaller angle of attack calibration range. In this case, the potentiometer measuring angle of attack was calibrated over a smaller range of $-30^{\circ}$ to $30^{\circ}$ which made the angle of attack reading more sensitive than those for the other tests. The aerodynamic coefficients for this data are tabulated in Appendix B.

The pressure distributions and coefficient data for other test conditions are in Appendix B.

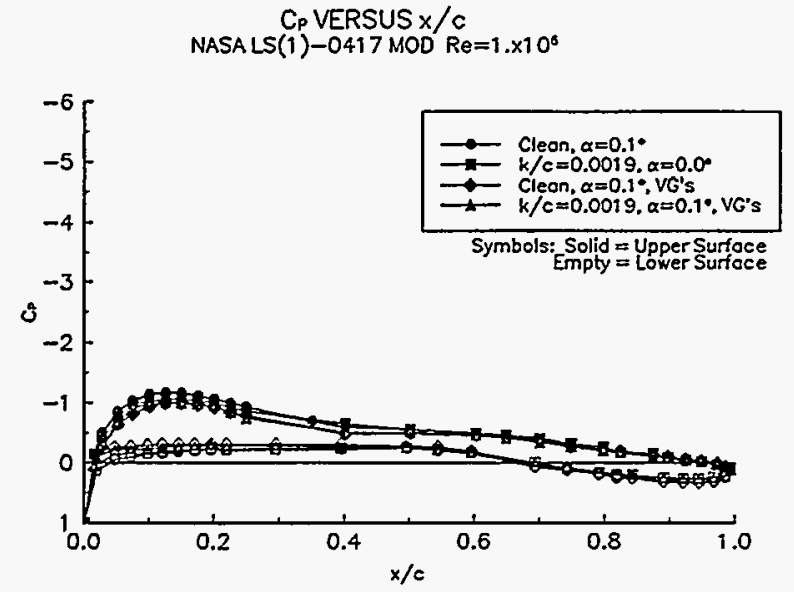

Figure 17. $C_{p}$ vs $x / c, \alpha=0^{\circ}$

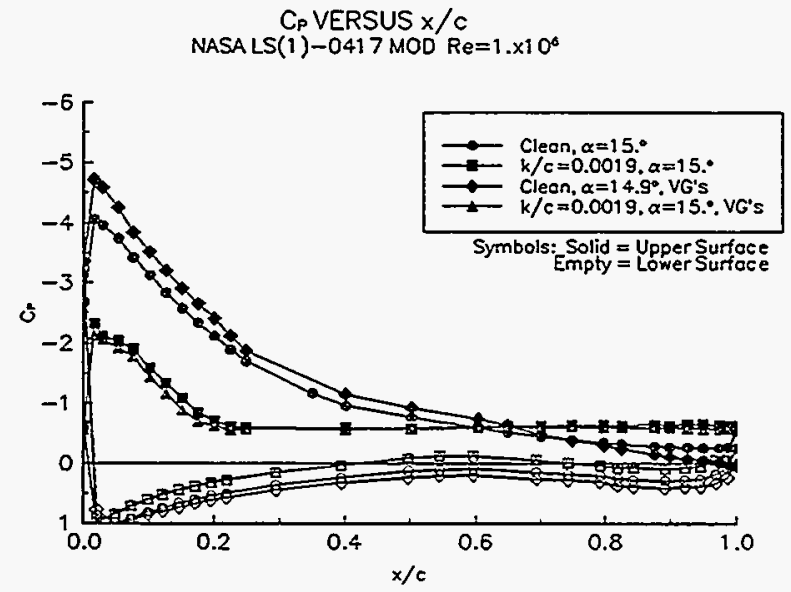

Figure 18. $C_{p}$ vs $x / c, \alpha=15^{\circ}$ 


\section{Summary}

A LS(1)-0417MOD model was installed in the OSU/AARL $7 \times 10$ subsonic wind tunnel and tested at three Reynolds numbers and with model clean, roughened, and with VGs. Table 1 is a summary of the aerodynamic coefficient data for the LS(1)-0417MOD.

Table 1. LS(1)-0417MOD Aerodynamic Parameters Summary

\begin{tabular}{||l|l|l|l|l||}
\hline \multicolumn{1}{|c|}{ CASE } & \multicolumn{1}{|c|}{ Re num } & $\mathrm{C}_{\operatorname{lmax}}$ & $\mathrm{dC} / \mathrm{d} \alpha$ & $\mathrm{C}_{\mathrm{mo}}$ \\
\hline \hline Clean & $1.0 \times 10^{6}$ & 1.51 & 0.099 & -0.071 \\
\hline Clean & $1.5 \times 10^{6}$ & 1.58 & 0.103 & -0.080 \\
\hline Clean & $2.0 \times 10^{6}$ & 1.63 & 0.107 & -0.080 \\
\hline $\mathrm{k} / \mathrm{c}=0.0019$ & $1.0 \times 10^{6}$ & 1.12 & 0.099 & -0.065 \\
\hline $\mathrm{k} / \mathrm{c}=0.0019$ & $1.5 \times 10^{6}$ & 1.14 & 0.098 & -0.067 \\
\hline $\mathrm{k} / \mathrm{c}=0.0019$ & $2.0 \times 10^{6}$ & 1.16 & 0.102 & -0.068 \\
\hline Clean VG's & $1.0 \times 10^{6}$ & 1.88 & 0.105 & -0.082 \\
\hline Clean VG's & $1.5 \times 10^{6}$ & 1.85 & 0.107 & -0.082 \\
\hline $\mathrm{k} / \mathrm{c}=0.0019$ VG's & $1.0 \times 10^{6}$ & 1.41 & 0.105 & -0.070 \\
\hline $\mathrm{k} / \mathrm{c}=0.0019$ VG's & $1.5 \times 10^{6}$ & 1.40 & 0.099 & -0.072 \\
\hline
\end{tabular}

For the clean LS(1)-0417MOD model, Reynolds number changes from 1 to 2 million did not a significant affect the maximum positive lift or the pitching moment at zero degrees angle of attack. However, the addition of leading edge grit roughness to the model reduced the maximum lift coefficient by $28 \%$ and caused a $16 \%$ change in the pitching moment at zero degrees angle of attack. Also, the lift curve slope showed slight increases with Reynolds number. Adding VGs on the model's upper surface at the 30\% chord station caused the maximum lift coefficient to increase by $25 \%$ in the clean cases and by about $26 \%$ for the roughened cases. The VGs apparently energized the boundary layer sufficiently to increase the lift curve slope and to delay stall to a higher angle of attack and, consequently, a higher maximum lift coefficient. However, the positive stall with VG's was more abrupt than the stall without VG's. 
Appendix A: Model and Surface Pressure Tap Coordinates 


\begin{tabular}{|c|c|c|c|}
\hline \multicolumn{4}{|c|}{$\begin{array}{l}\text { Table Al. LS(1)-0417MOD Measured Model Coordinates } \\
\qquad 18 \text { inch desired chord }\end{array}$} \\
\hline $\begin{array}{l}\text { Chord Station } \\
\text { (in) }\end{array}$ & $\begin{array}{l}\text { Lower Ordinate } \\
\text { (in) }\end{array}$ & $\begin{array}{l}\text { Chord Station } \\
\text { (in) }\end{array}$ & $\begin{array}{l}\text { Upper Ordinate } \\
\text { (in) }\end{array}$ \\
\hline 0.000 & -0.003 & 0.000 & -0.003 \\
\hline 0.004 & -0.027 & 0.001 & 0.146 \\
\hline 0.013 & -0.069 & 0.003 & 0.165 \\
\hline 0.034 & -0.145 & 0.005 & 0.175 \\
\hline 0.058 & -0.195 & 0.010 & 0.200 \\
\hline 0.118 & -0.277 & 0.022 & 0.245 \\
\hline 0.190 & -0.344 & 0.052 & 0.330 \\
\hline 0.288 & -0.407 & 0.081 & 0.389 \\
\hline 0.359 & -0.445 & 0.147 & 0.496 \\
\hline 0.445 & -0.485 & 0.227 & 0.602 \\
\hline 0.580 & -0.539 & 0.330 & 0.718 \\
\hline 0.710 & -0.585 & 0.407 & 0.795 \\
\hline 0.864 & -0.632 & 0.498 & 0.876 \\
\hline 1.092 & -0.700 & 0.636 & 0.986 \\
\hline 1.316 & -0.746 & 0.770 & 1.078 \\
\hline 1.527 & -0.788 & 0.925 & 1.172 \\
\hline 1.690 & -0.818 & 1.077 & 1.253 \\
\hline 1.855 & -0.846 & 1.195 & 1.311 \\
\hline 2.364 & -0.922 & 1.389 & 1.397 \\
\hline 2.917 & -0.989 & 1.601 & 1.479 \\
\hline 3.297 & -1.027 & 1.768 & 1.536 \\
\hline 3.754 & -1.064 & 1.933 & 1.587 \\
\hline 4.834 & -1.122 & 2.067 & 1.625 \\
\hline 5.782 & -1.146 & 2.447 & 1.715 \\
\hline 6.668 & -1.148 & 3.381 & 1.863 \\
\hline 7.448 & -1.132 & 3.840 & 1.901 \\
\hline 8.461 & -1.092 & 4.245 & 1.920 \\
\hline
\end{tabular}

A-2 


\begin{tabular}{|c|c|c|c|}
\hline \multicolumn{4}{|c|}{$\begin{array}{c}\text { Table A1. LS(1)-0417MOD Measured Model Coordinates } \\
18 \text { inch desired chord }\end{array}$} \\
\hline $\begin{array}{l}\text { Chord Station } \\
\text { (in) }\end{array}$ & $\begin{array}{l}\text { Lower Ordinate } \\
\quad \text { (in) }\end{array}$ & $\begin{array}{l}\text { Chord Station } \\
\text { (in) }\end{array}$ & $\begin{array}{l}\text { Upper Ordinate } \\
\text { (in) }\end{array}$ \\
\hline 9.318 & -1.037 & 4.924 & 1.927 \\
\hline 10.250 & -0.943 & 5.869 & 1.905 \\
\hline 11.131 & -0.819 & 6.756 & 1.861 \\
\hline 12.905 & -0.504 & 7.534 & 1.814 \\
\hline 13.820 & -0.330 & 8.546 & 1.743 \\
\hline 14.514 & -0.207 & 9.398 & 1.673 \\
\hline 14.980 & -0.137 & 10.327 & 1.579 \\
\hline 15.487 & -0.070 & 11.202 & 1.466 \\
\hline 15.854 & -0.027 & 12.958 & 1.174 \\
\hline 16.293 & 0.007 & 13.860 & 0.999 \\
\hline 16.829 & 0.032 & 14.543 & 0.859 \\
\hline 17.177 & 0.029 & 15.006 & 0.762 \\
\hline 17.389 & 0.018 & 15.509 & 0.655 \\
\hline 17.457 & 0.013 & 15.869 & 0.577 \\
\hline 17.551 & 0.004 & 16.338 & 0.473 \\
\hline 17.618 & -0.003 & 16.829 & 0.362 \\
\hline 17.703 & -0.013 & 17.171 & 0.280 \\
\hline 17.781 & -0.023 & 17.293 & 0.250 \\
\hline 17.802 & -0.025 & 17.381 & 0.227 \\
\hline 17.833 & -0.029 & 17.448 & 0.209 \\
\hline \multirow[t]{4}{*}{17.953} & -0.037 & 17.541 & 0.183 \\
\hline & & 17.605 & 0.164 \\
\hline & & 17.686 & 0.138 \\
\hline & & 17.953 & 0.058 \\
\hline \multicolumn{4}{|c|}{ End of Table A1 } \\
\hline
\end{tabular}




\begin{tabular}{|c|c|c|}
\hline Tap Number & Chord Station & Ordinate \\
\hline 1 & 1.0000 & -0.0035 \\
\hline 2 & 0.9847 & -0.0009 \\
\hline 3 & 0.9681 & 0.0006 \\
\hline 4 & 0.9448 & 0.0016 \\
\hline 5 & 0.9209 & 0.0013 \\
\hline 6 & 0.8915 & -0.0003 \\
\hline 7 & 0.8694 & -0.0025 \\
\hline 8 & 0.8435 & -0.0058 \\
\hline 9 & 0.8188 & -0.0093 \\
\hline 10 & 0.7919 & -0.0138 \\
\hline 11 & 0.7432 & -0.0227 \\
\hline 12 & 0.6941 & -0.0320 \\
\hline 13 & 0.6444 & -0.0408 \\
\hline 14 & 0.5955 & -0.0487 \\
\hline 15 & 0.5448 & -0.0550 \\
\hline 16 & 0.4967 & -0.0590 \\
\hline 17 & 0.3951 & -0.0632 \\
\hline 18 & 0.2951 & -0.0631 \\
\hline 19 & 0.2462 & -0.0614 \\
\hline 20 & 0.2196 & -0.0598 \\
\hline 21 & 0.1963 & -0.0581 \\
\hline 22 & 0.1714 & -0.0559 \\
\hline 23 & 0.1461 & -0.0531 \\
\hline 24 & 0.1207 & -0.0497 \\
\hline 25 & 0.0977 & -0.0461 \\
\hline 26 & 0.0721 & -0.0410 \\
\hline 27 & 0.0467 & -0.0344 \\
\hline 28 & 0.0187 & -0.0238 \\
\hline
\end{tabular}

A-4 


\begin{tabular}{|c|c|c|}
\hline \multicolumn{3}{|c|}{ Table A2. LS(1)-0417MOD Surface Pressure Tap } \\
\hline Tap Number & Chord Station & Ordinate \\
\hline 29 & 0.0000 & 0.0006 \\
\hline 30 & 0.0152 & 0.0365 \\
\hline 31 & 0.0284 & 0.0492 \\
\hline 32 & 0.0517 & 0.0651 \\
\hline 33 & 0.0751 & 0.0767 \\
\hline 34 & 0.1012 & 0.0863 \\
\hline 35 & 0.1263 & 0.0934 \\
\hline 36 & 0.1508 & 0.0985 \\
\hline 37 & 0.1761 & 0.1024 \\
\hline 38 & 0.2009 & 0.1050 \\
\hline 39 & 0.2264 & 0.1066 \\
\hline 40 & 0.2503 & 0.1073 \\
\hline 41 & 0.3009 & 0.1068 \\
\hline 42 & 0.3513 & 0.1046 \\
\hline 43 & 0.4018 & 0.1017 \\
\hline 44 & 0.4517 & 0.0984 \\
\hline 45 & 0.5016 & 0.0947 \\
\hline 46 & 0.5491 & 0.0905 \\
\hline 47 & 0.6043 & 0.0842 \\
\hline 48 & 0.6500 & 0.0776 \\
\hline 49 & 0.7009 & 0.0689 \\
\hline 50 & 0.7494 & 0.0596 \\
\hline 51 & 0.7980 & 0.0497 \\
\hline 52 & 0.8246 & 0.0442 \\
\hline 53 & 0.8533 & 0.0381 \\
\hline 54 & 0.8751 & 0.0333 \\
\hline 55 & 0.8979 & 0.0284 \\
\hline 56 & 0.9250 & 0.0223 \\
\hline
\end{tabular}

A-5 


\begin{tabular}{|c|c|c||}
\hline \multicolumn{3}{||c|}{ Table A2. LS(1)-0417MOD Surface Pressure Taps } \\
\hline \hline Tap Number & Chord Station & Ordinate \\
\hline 57 & 0.9494 & 0.0167 \\
58 & 0.9726 & 0.0112 \\
59 & 0.9857 & 0.0069 \\
\hline \multicolumn{3}{|c|}{ End of Table A2 } \\
\hline
\end{tabular}


Appendix B: Integrated Coefficients and Pressure Distributions 


\section{List of Tables}

Page

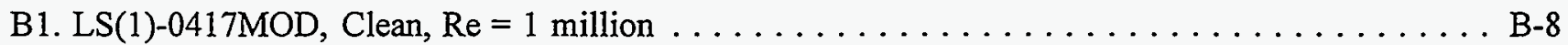

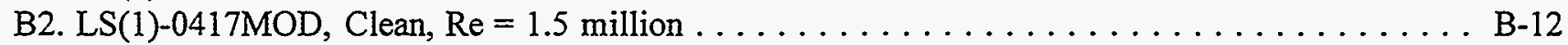

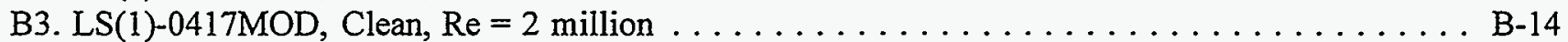

B4. LS(1)-0417MOD, LEGR $\mathrm{k} / \mathrm{c}=0.0019, \mathrm{Re}=1$ million $\ldots \ldots \ldots \ldots \ldots \ldots \ldots \ldots$. . . . . . . . . . .

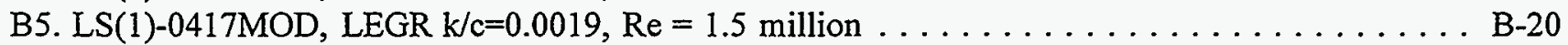

B6. LS(1)-0417MOD, LEGR $\mathrm{k} / \mathrm{c}=0.0019, \mathrm{Re}=2$ million . . . . . . . . . . . . . . . . . . . B B-22

B7. LS(1)-0417MOD, LEGR k/c=0.0019, VGs, Re $=1$ million . . . . . . . . . . . . . . . . . B-24

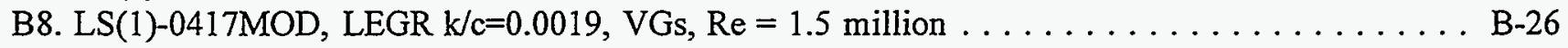

B9. LS(1)-0417MOD, Clean, VGs, $R e=1$ million $\ldots \ldots \ldots \ldots \ldots \ldots \ldots \ldots \ldots \ldots$ B-28

B10. LS(1)-0417MOD, Clean, VGs, $R e=1.5$ million $\ldots \ldots \ldots \ldots \ldots \ldots \ldots \ldots \ldots \ldots \ldots$ B $\ldots \ldots \ldots$

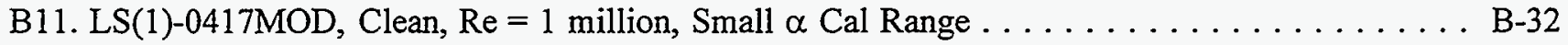

B12. LS(1)-0417MOD, Clean, $R e=1.5$ million, Small $\alpha$ Cal Range $\ldots \ldots \ldots \ldots \ldots \ldots$. . . . . . . . 
Steady State Pressure Distributions $\operatorname{Re}=1.0$ million $\ldots \ldots \ldots \ldots \ldots \ldots \ldots \ldots$

1. $\alpha=-60^{\circ}$

B-37

2. $\alpha=-58^{\circ}$

B-37

3. $\alpha=-54^{\circ}$

B-37

4. $\alpha=-50^{\circ}$

B-37

5. $\alpha=-46^{\circ}$

B-38

6. $\alpha=-42^{\circ}$

B-38

7. $\alpha=-38^{\circ}$

8. $\alpha=-34^{\circ}$

B-38

9. $\alpha=-30^{\circ}$

B-38

10. $\alpha=-26^{\circ}$

B-39

11. $\alpha=-22^{\circ}$

B-39

12. $\alpha=-20^{\circ}$

B-39

13. $\alpha=-18^{\circ}$

B-39

14. $\alpha=-16^{\circ}$

B- 40

15. $\alpha=-14^{\circ}$

B- 40

16. $\alpha=-12^{\circ}$

B-40

17. $\alpha=-10^{\circ}$

B- 40

18. $\alpha=-8^{\circ}$

B-41

19. $\alpha=-6^{\circ}$

B-41

20. $\alpha=-4^{\circ}$

B-41

21. $\alpha=-2^{\circ}$

B-41

22. $\alpha=0^{\circ}$

23. $\alpha=2^{\circ}$

24. $\alpha=4^{\circ}$

25. $\alpha=6^{\circ}$

26. $\alpha=8^{\circ}$

B- 42

B-42

B-42

B- 42

B- 43

27. $\alpha=10^{\circ}$

B-43

28. $\alpha=11^{\circ}$

B- 43

29. $\alpha=12^{\circ}$

B-43

30. $\alpha=13^{\circ}$

B- 44

31. $\alpha=14^{\circ}$

B- 44

32. $\alpha=15^{\circ}$

B- 44

33. $\alpha=16^{\circ}$

B- 44

34. $\alpha=17^{\circ}$

B- 45

35. $\alpha=18^{\circ}$

B-45

36. $\alpha=19^{\circ}$

B- 45

37. $\alpha=20^{\circ}$

B-45

38. $\alpha=22^{\circ}$

B-46

39. $\alpha=24^{\circ}$

B-46

40. $\alpha=26^{\circ}$

B-46

41. $\alpha=28^{\circ}$

B- 46

42. $\alpha=30^{\circ}$

B- 47

43. $\alpha=32^{\circ}$

B- 47

44. $\alpha=34^{\circ}$ 


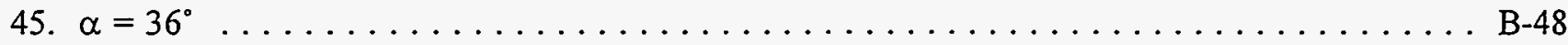

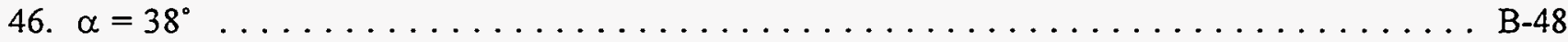

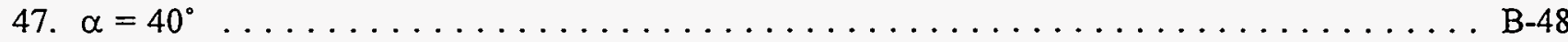

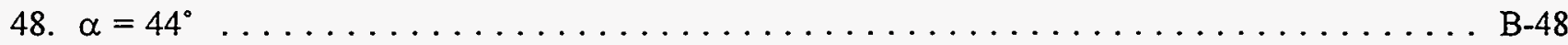

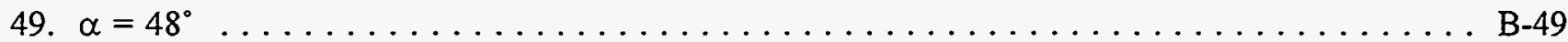

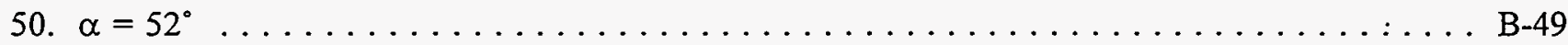

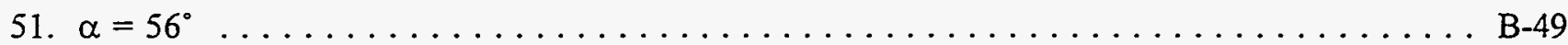

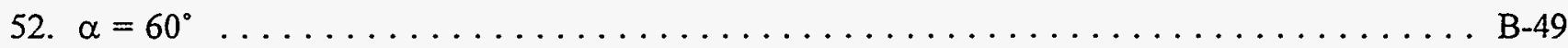

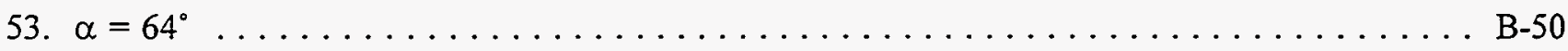

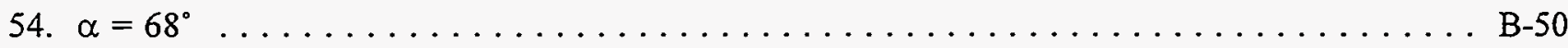

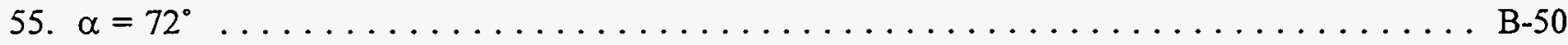

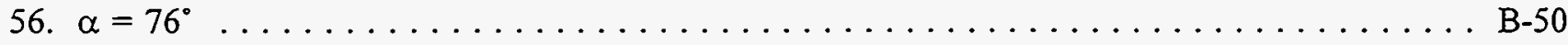

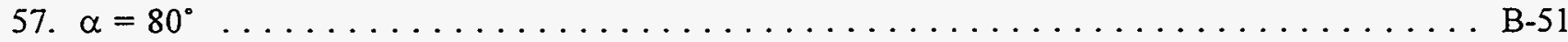

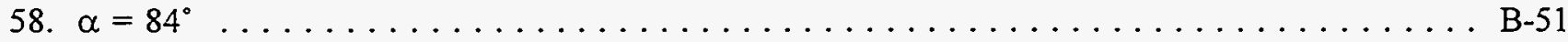

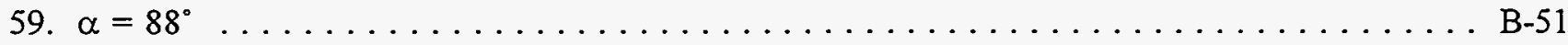

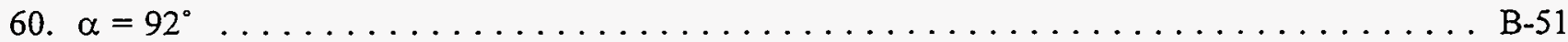

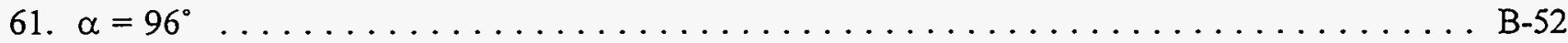

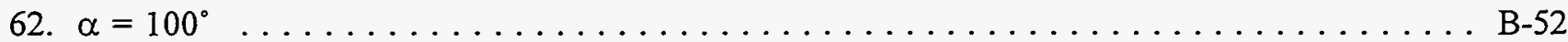

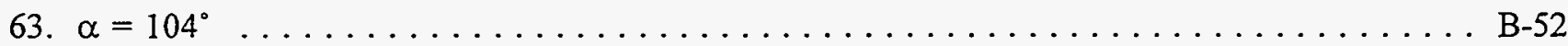

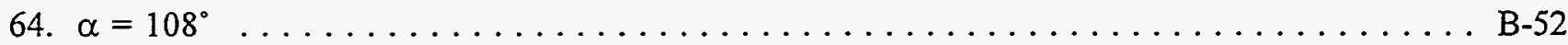

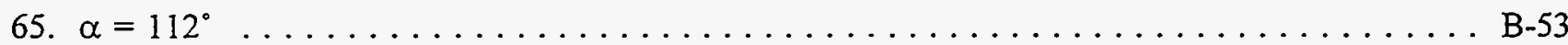

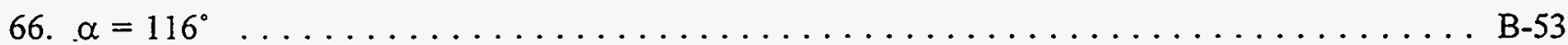

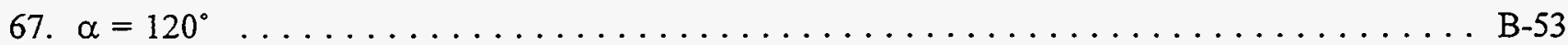

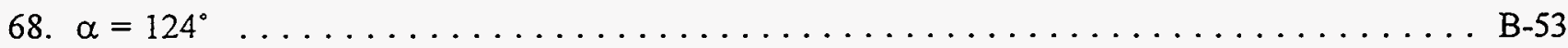

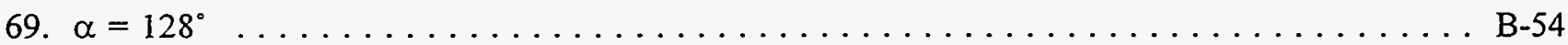

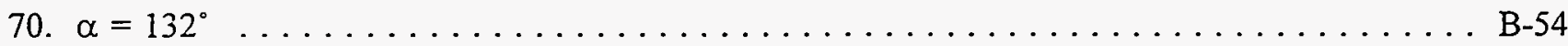

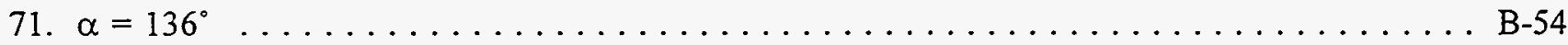

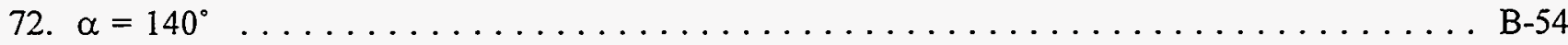

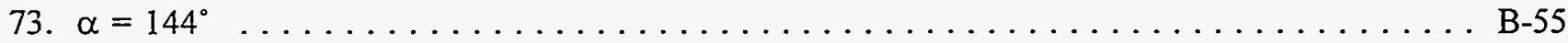

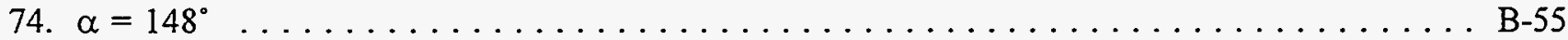

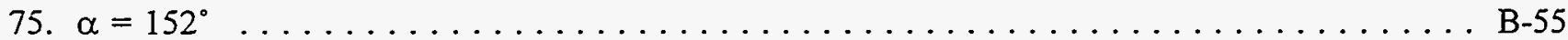

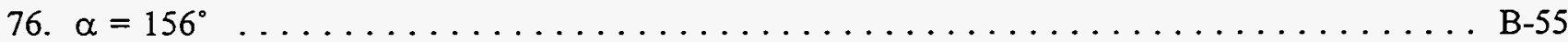

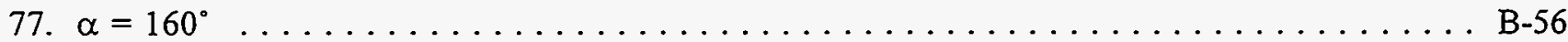

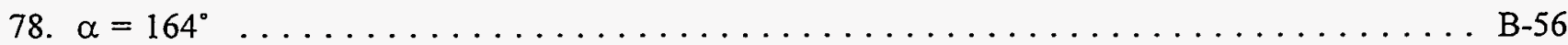

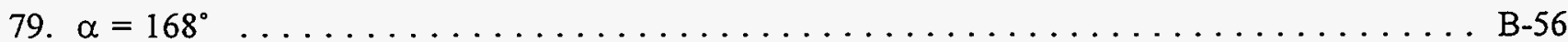

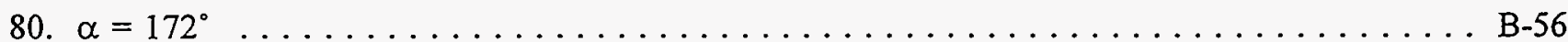

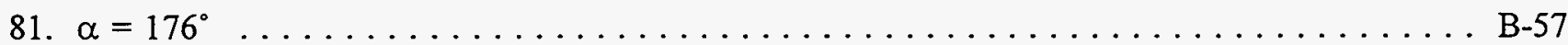

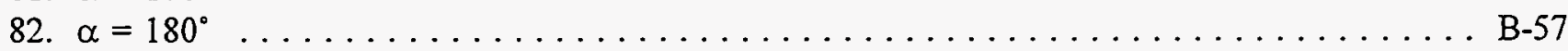

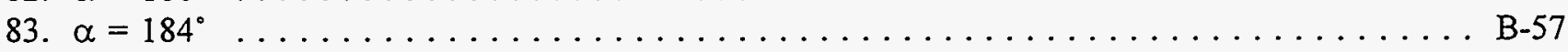

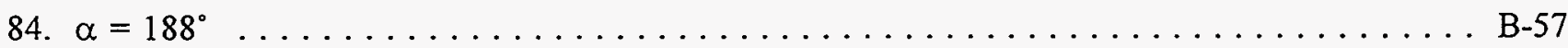

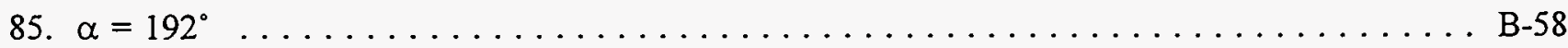

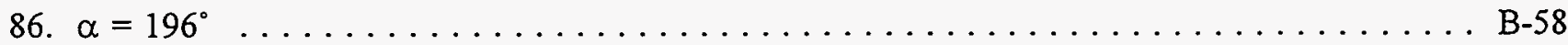

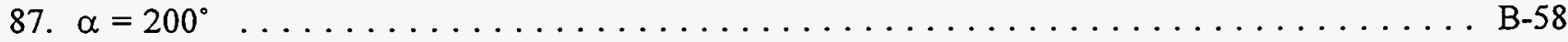

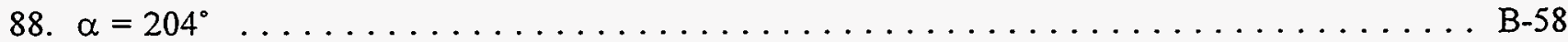

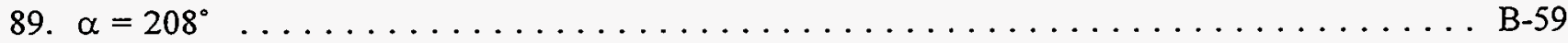

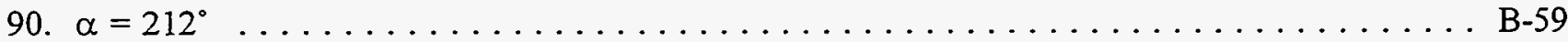

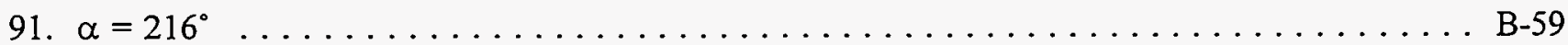

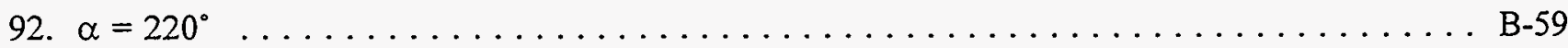




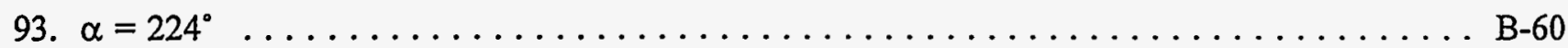

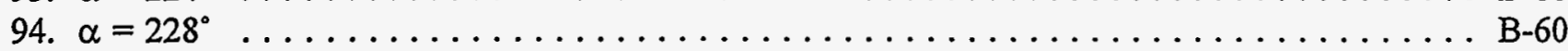

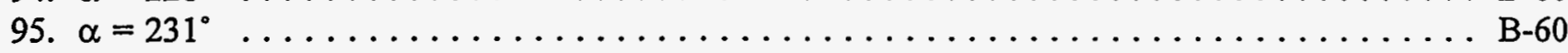

Steady State Pressure Distributions, VGs, $\operatorname{Re}=1.0$ million $\ldots \ldots \ldots \ldots \ldots \ldots \ldots \ldots$ B-61

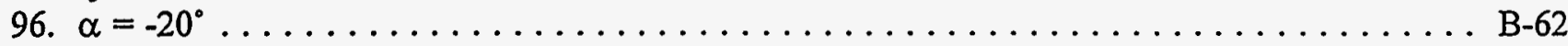

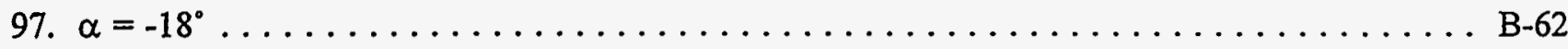

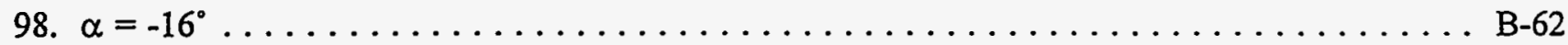

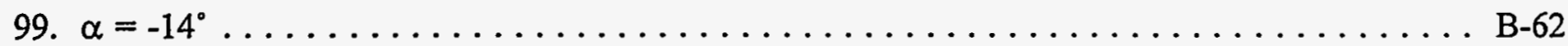

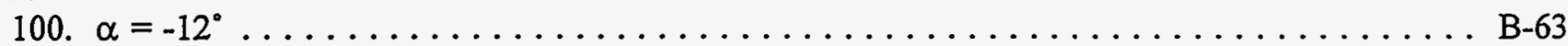

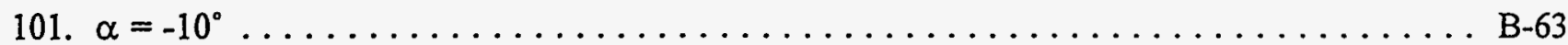

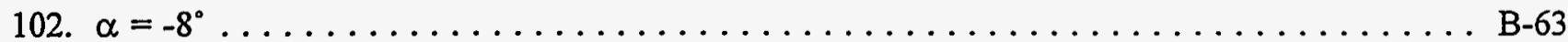

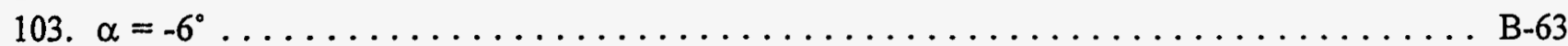

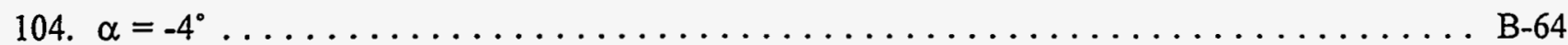

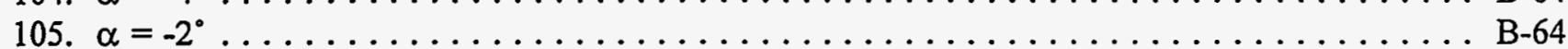

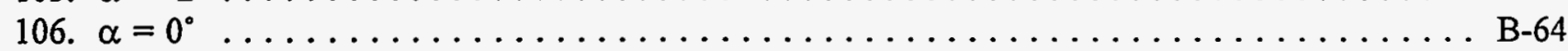

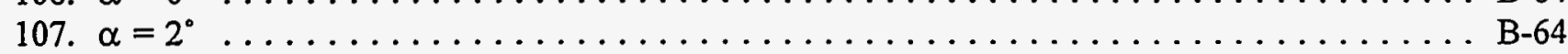

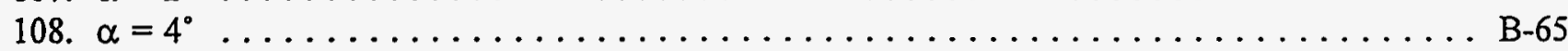

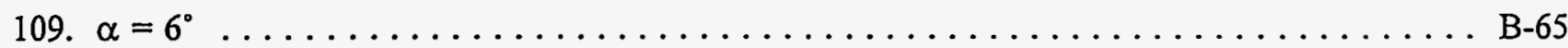

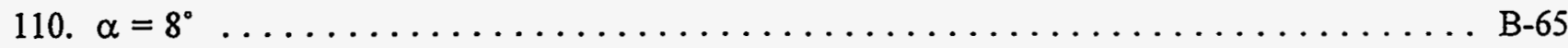

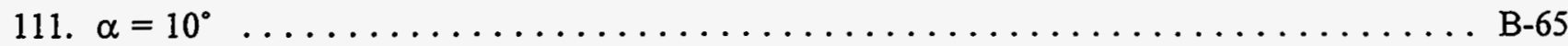

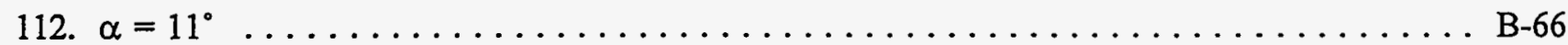

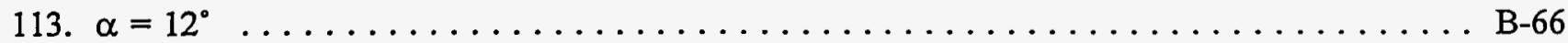

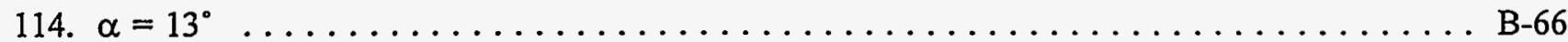

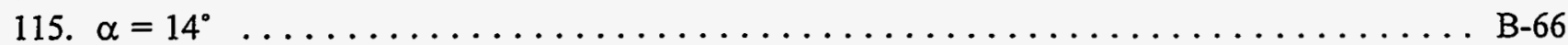

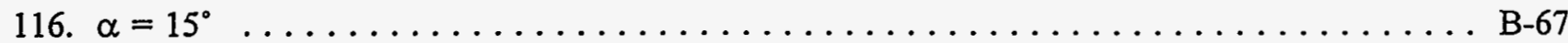

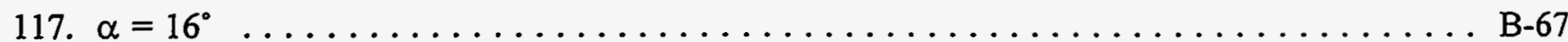

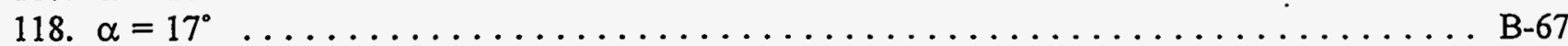

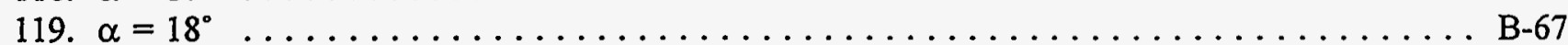

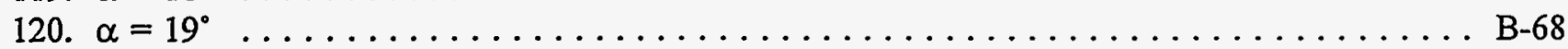

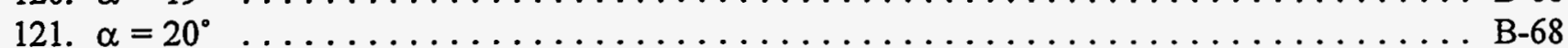

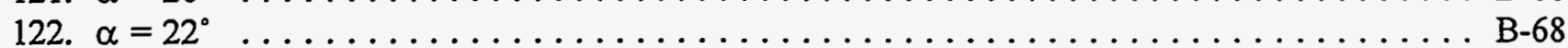

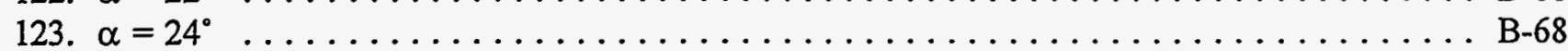

Steady State Pressure Distributions, $\operatorname{Re}=1.5$ million $\ldots \ldots \ldots \ldots \ldots \ldots \ldots \ldots \ldots$

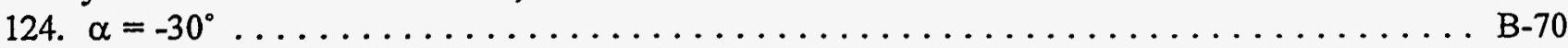

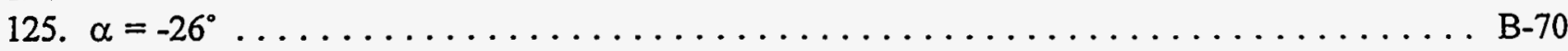

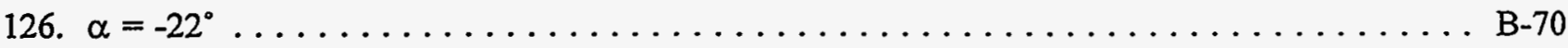

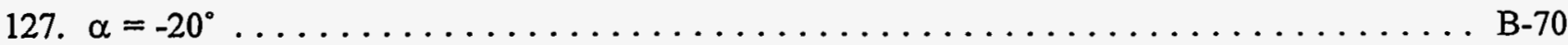

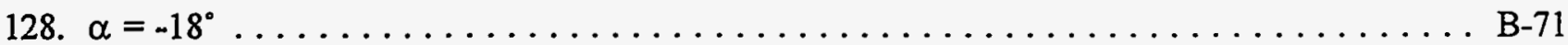

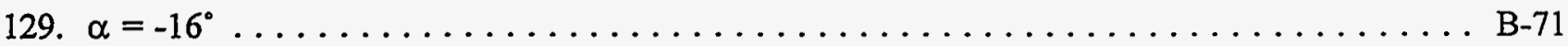

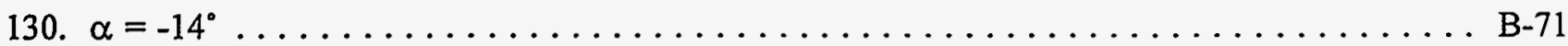

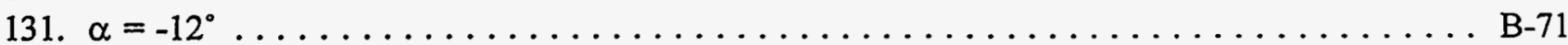

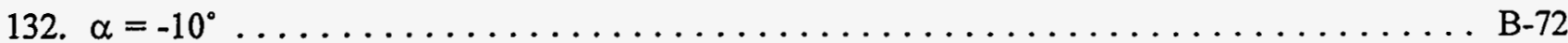

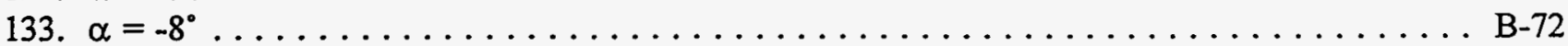

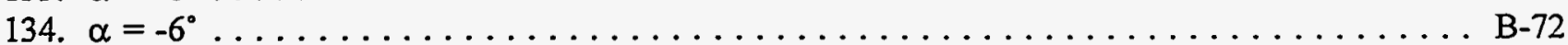

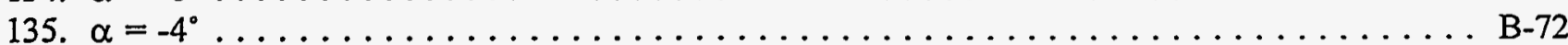

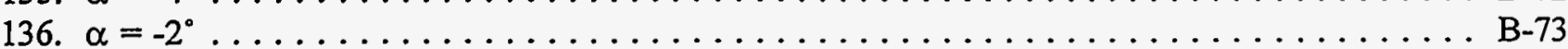

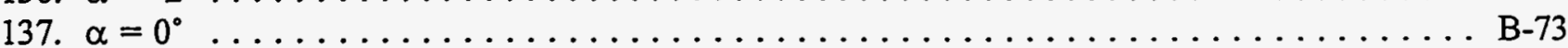

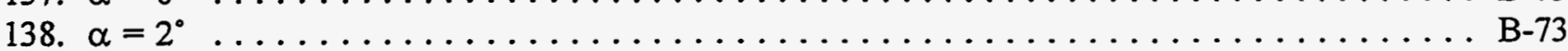




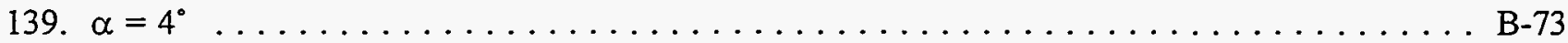

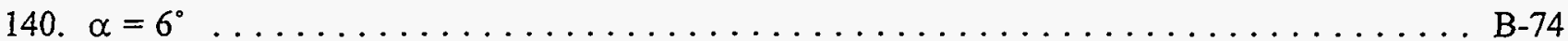

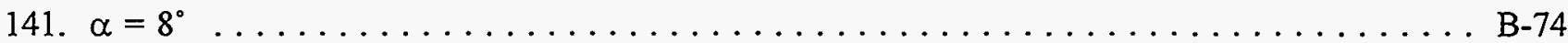

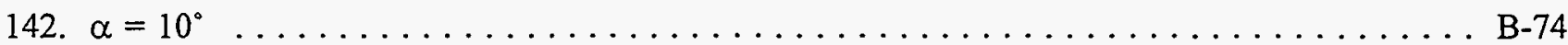

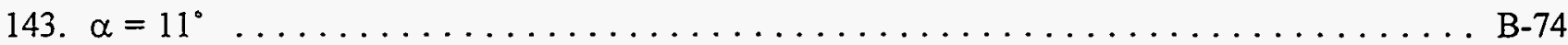

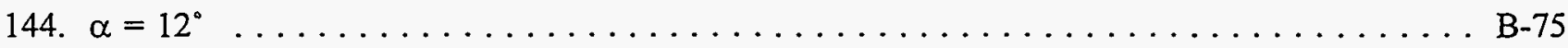

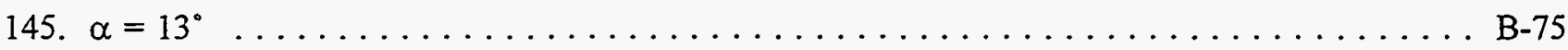

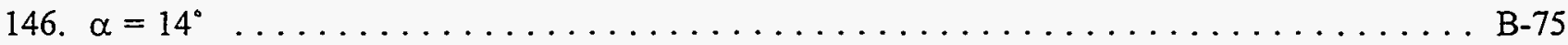

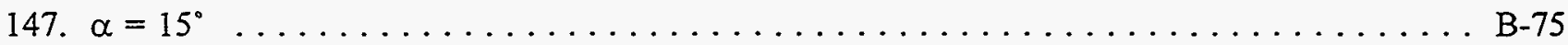

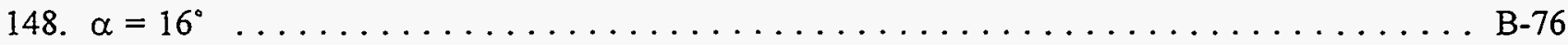

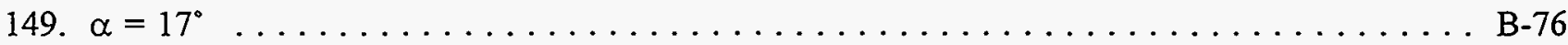

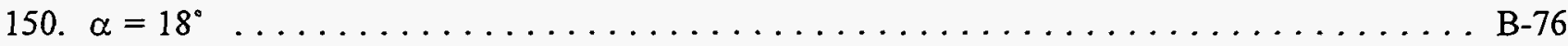

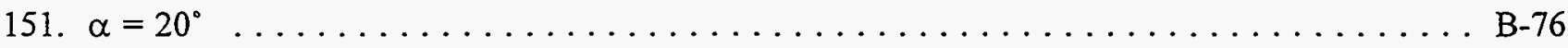

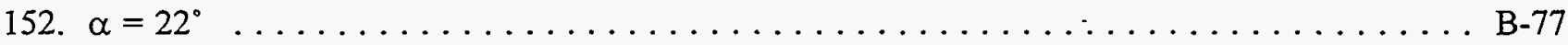

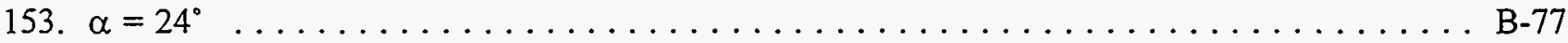

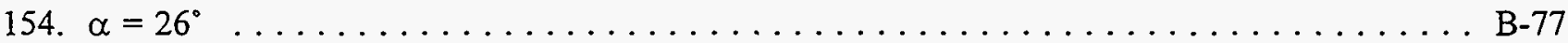

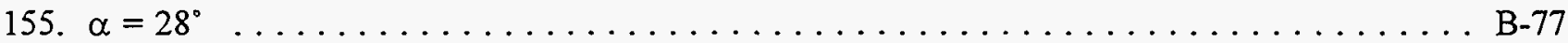

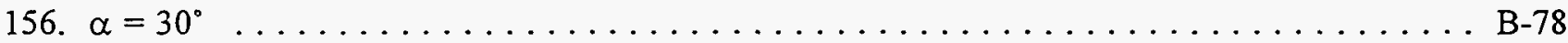

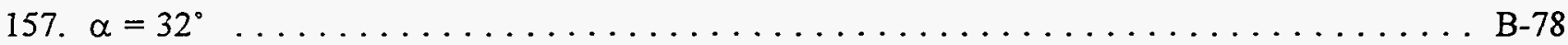

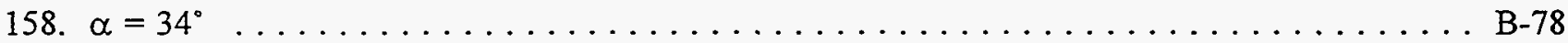

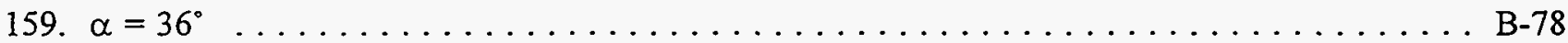

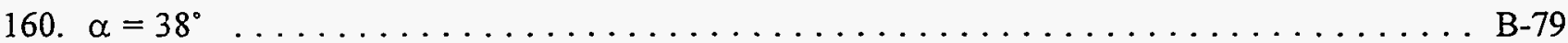

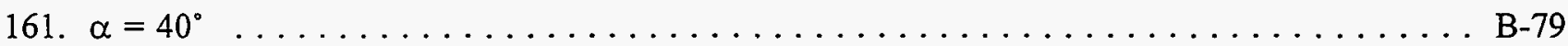

Steady State Pressure Distributions $\mathrm{Re}=2.0$ million $\ldots \ldots \ldots \ldots \ldots \ldots \ldots \ldots \ldots$

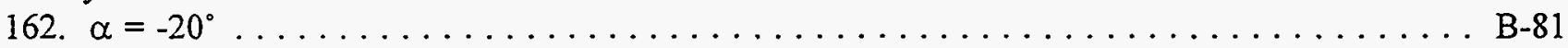

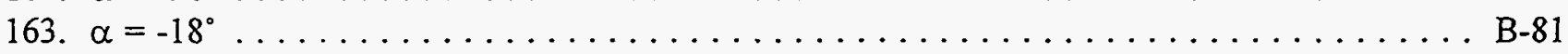

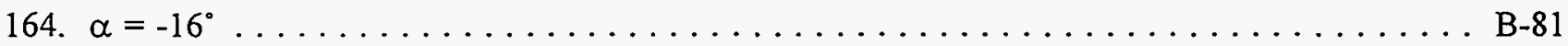

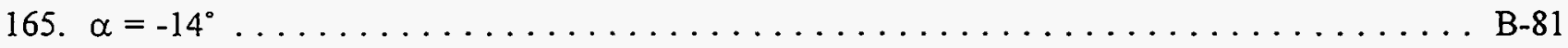

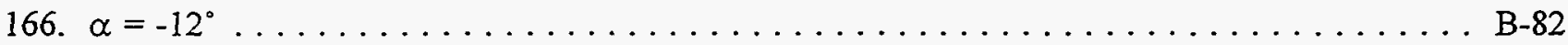

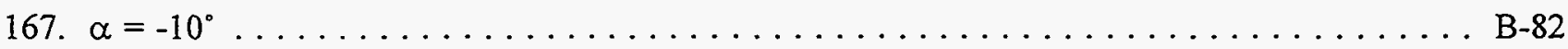

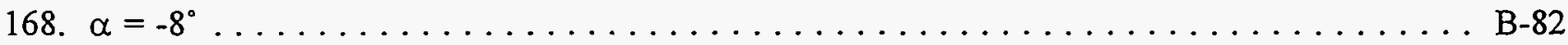

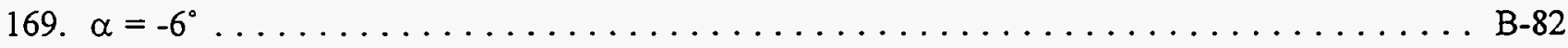

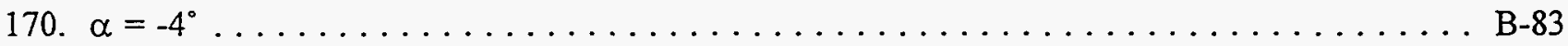

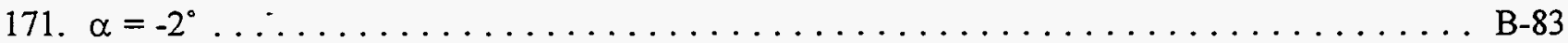

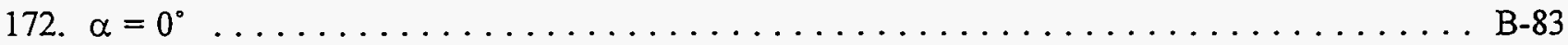

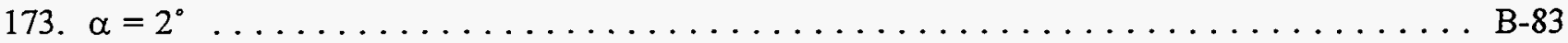

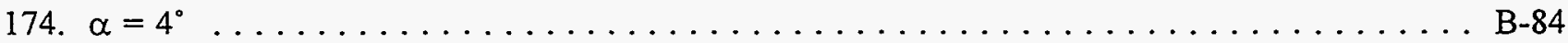

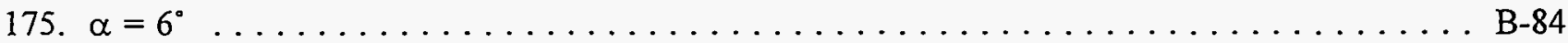

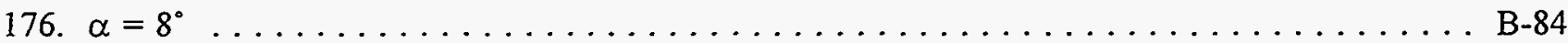

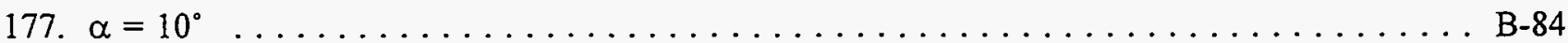

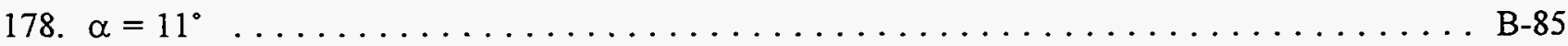

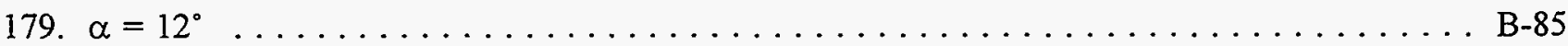

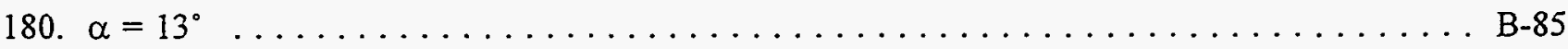

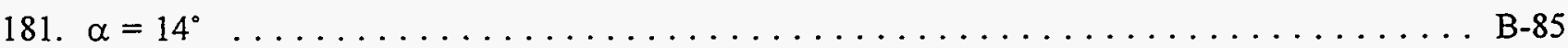

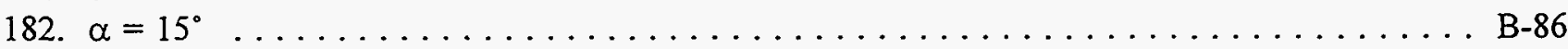

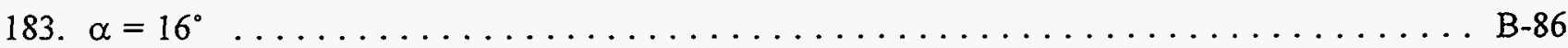

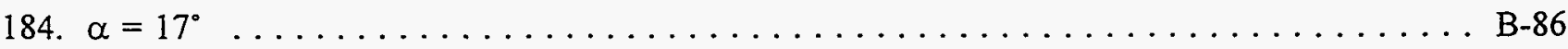

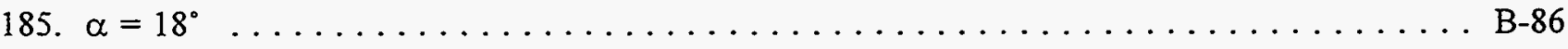


186. $\alpha=19^{\circ}$ B-87

187. $\alpha=20^{\circ}$

188. $\alpha=22^{\circ}$

189. $\alpha=24^{\circ}$

B-87

190. $\alpha=26^{\circ}$

191. $\alpha=28^{\circ}$

192. $\alpha=30^{\circ}$

193. $\alpha=32^{\circ}$

194. $\alpha=34^{\circ}$

B-87

195. $\alpha=36^{\circ}$

B-88

196. $\alpha=38^{\circ}$

B-88

197. $\alpha=40^{\circ}$

B-88

B-88

B- 89

B-89

B-89

B-89 


\begin{tabular}{|c|c|c|c|c|c|}
\hline \multicolumn{6}{|c|}{ Table B1. LS(1)-0417MOD, Clean, $\mathrm{Re}=1$ million } \\
\hline RUN & $\mathrm{AOA}$ & $\mathrm{C}_{1}$ & $\mathrm{C}_{\mathrm{dp}}$ & $\mathrm{C}_{\mathrm{m} / \mathrm{s}}$ & $\operatorname{Re} \times 10^{-6}$ \\
\hline 112 & -29.9 & -0.79 & 0.5651 & 0.1097 & 1.00 \\
\hline 113 & -25.8 & -0.61 & 0.4151 & 0.0688 & 1.00 \\
\hline 114 & -25.9 & -0.58 & 0.3992 & 0.0590 & 1.00 \\
\hline 115 & -21.9 & -0.40 & 0.2765 & 0.0273 & 1.00 \\
\hline 116 & -17.9 & -0.30 & 0.2069 & 0.0142 & 0.99 \\
\hline 117 & -15.9 & -0.27 & 0.1836 & 0.0149 & 1.01 \\
\hline 118 & -13.9 & -0.24 & 0.1581 & 0.0111 & 1.00 \\
\hline 119 & -11.9 & -0.71 & 0.0444 & -0.0514 & 1.00 \\
\hline 120 & -9.8 & -0.50 & 0.0299 & -0.0640 & 1.00 \\
\hline 121 & -7.8 & -0.29 & 0.0177 & -0.0691 & 1.00 \\
\hline 122 & -6.0 & -0.13 & 0.0099 & -0.0637 & 0.99 \\
\hline 123 & -3.9 & 0.05 & 0.0019 & -0.0627 & 1.00 \\
\hline 124 & -1.9 & 0.27 & -.0033 & -0.0678 & 1.00 \\
\hline 125 & 0.1 & 0.48 & -.0072 & -0.0706 & $\cdot 1.00$ \\
\hline 126 & 2.2 & 0.69 & -.0070 & -0.0684 & 1.00 \\
\hline 127 & 4.0 & 0.87 & -.0056 & -0.0692 & 1.00 \\
\hline 128 & 6.1 & 1.06 & -.0038 & -0.0664 & 1.00 \\
\hline 129 & 8.2 & 1.22 & 0.0028 & -0.0612 & 1.00 \\
\hline 130 & 10.2 & 1.39 & 0.0046 & -0.0536 & 1.00 \\
\hline 131 & 11.2 & 1.44 & 0.0105 & -0.0483 & 1.00 \\
\hline 132 & 11.9 & 1.48 & 0.0137 & -0.0460 & 1.00 \\
\hline 133 & 13.0 & 1.51 & 0.0227 & -0.0417 & 1.00 \\
\hline 134 & 14.0 & 1.49 & 0.0593 & -0.0511 & 0.99 \\
\hline 135 & 15.0 & 1.51 & 0.0813 & -0.0541 & 0.99 \\
\hline 136 & 16.1 & 1.46 & 0.1054 & -0.0523 & 0.98 \\
\hline 137 & 16.9 & 1.45 & 0.1241 & -0.0512 & 0.98 \\
\hline 138 & 17.9 & 1.43 & 0.1411 & -0.0577 & 1.00 \\
\hline
\end{tabular}




\begin{tabular}{|c|c|c|c|c|c|}
\hline \multicolumn{6}{|c|}{ Table B1. LS(1)-0417MOD, Clean, $\mathrm{Re}=1$ million } \\
\hline RUN & AOA & $\mathrm{C}_{1}$ & $\mathrm{C}_{\mathrm{dp}}$ & $\mathrm{C}_{\mathrm{m} / \mathrm{t}}$ & $\operatorname{Re} \times 10^{-6}$ \\
\hline 139 & 18.9 & 1.44 & 0.1638 & -0.0621 & 1.00 \\
\hline 140 & 20.0 & 1.46 & 0.1996 & -0.1036 & 0.99 \\
\hline 141 & 22.1 & 1.40 & 0.2357 & -0.1190 & 1.00 \\
\hline 142 & 24.0 & 1.31 & 0.2701 & -0.1287 & 0.99 \\
\hline 143 & 26.1 & 1.08 & 0.3038 & -0.1423 & 1.00 \\
\hline 144 & 28.2 & 1.12 & 0.3670 & -0.1594 & 0.99 \\
\hline 145 & 30.0 & 0.89 & 0.5474 & -0.1825 & 1.00 \\
\hline 146 & 32.0 & 0.94 & 0.6135 & -0.1985 & 1.00 \\
\hline 147 & 34.1 & 0.93 & 0.6520 & -0.2001 & 1.00 \\
\hline 148 & 36.2 & 1.25 & 0.9319 & -0.2931 & 1.00 \\
\hline 149 & 38.0 & 1.26 & 1.0082 & -0.3127 & 1.00 \\
\hline 150 & 40.0 & 1.33 & 1.1364 & -0.3447 & 0.89 \\
\hline 151 & 44.2 & 1.39 & 1.3667 & -0.4016 & 0.86 \\
\hline 152 & 48.0 & 1.31 & 1.4531 & -0.4090 & 0.82 \\
\hline 153 & 52.0 & 1.35 & 1.7181 & -0.4772 & 0.81 \\
\hline 154 & 56.0 & 1.25 & 1.8152 & -0.4884 & 0.81 \\
\hline 155 & 60.2 & 1.19 & 2.0100 & -0.5310 & 0.80 \\
\hline 156 & 64.0 & 1.08 & 2.1204 & -0.5562 & 0.78 \\
\hline 157 & 68.1 & 0.94 & 2.2310 & -0.5849 & 0.77 \\
\hline 158 & 72.0 & 0.80 & 2.2325 & -0.5788 & 0.74 \\
\hline 159 & 76.1 & 0.64 & 2.2805 & -0.5936 & 0.73 \\
\hline 160 & 79.9 & 0.53 & 2.4723 & -0.6421 & 0.73 \\
\hline 161 & 84.1 & 0.35 & 2.4377 & -0.6523 & 0.71 \\
\hline 162 & 87.9 & 0.22 & 2.6610 & -0.7007 & 0.69 \\
\hline 163 & 92.1 & 0.02 & 2.4182 & -0.6662 & 0.70 \\
\hline 164 & 95.7 & -0.10 & 2.4400 & -0.6757 & 0.69 \\
\hline 165 & 100.1 & -0.29 & 2.3237 & -0.6765 & 0.70 \\
\hline
\end{tabular}




\begin{tabular}{|c|c|c|c|c|c|}
\hline \multicolumn{6}{|c|}{ Table B1. LS(1)-0417MOD, Clean, $R e=1$ million } \\
\hline RUN & $\mathrm{AOA}$ & $\mathrm{C}_{1}$ & $\mathrm{C}_{\mathrm{dp}}$ & $\mathrm{C}_{\mathrm{m} / 4}$ & $\operatorname{Re} \times 10^{-6}$ \\
\hline 166 & 104.2 & -0.44 & 2.3826 & -0.6824 & 0.69 \\
\hline 167 & 108.0 & -0.60 & 2.3449 & -0.7174 & 0.69 \\
\hline 168 & 112.1 & -0.76 & 2.2704 & -0.7271 & 0.70 \\
\hline 169 & 116.3 & -0.85 & 2.0492 & -0.7001 & 0.71 \\
\hline 170 & 120.4 & -0.88 & 1.7661 & -0.6356 & 0.73 \\
\hline 171 & 123.7 & -0.99 & 1.7734 & -0.6522 & 0.73 \\
\hline 172 & 128.1 & -1.06 & 1.6170 & -0.6374 & 0.75 \\
\hline 173 & 132.2 & -1.26 & 1.5870 & -0.6547 & 0.77 \\
\hline 174 & 135.8 & -1.19 & 1.3838 & -0.6086 & 0.79 \\
\hline 175 & 140.0 & -1.15 & 1.1450 & -0.5688 & 0.78 \\
\hline 176 & 144.3 & -0.97 & 0.8381 & -0.4096 & 0.82 \\
\hline 178 & 152.1 & -0.80 & 0.5259 & -0.3918 & 0.98 \\
\hline 179 & 156.0 & -0.68 & 0.3901 & -0.3350 & 1.00 \\
\hline 180 & 160.1 & -0.62 & 0.2962 & -0.2980 & 0.99 \\
\hline 181 & 164.1 & -0.78 & 0.2292 & -0.3584 & 1.00 \\
\hline 182 & 167.9 & -0.91 & 0.1249 & -0.4385 & 1.01 \\
\hline 183 & 172.1 & -0.60 & 0.0559 & -0.2607 & 0.98 \\
\hline 184 & 176.3 & -0.08 & 0.0299 & -0.0688 & 1.00 \\
\hline 185 & 180.1 & 0.34 & 0.0304 & 0.1299 & 1.00 \\
\hline 186 & 183.9 & 0.67 & 0.0472 & 0.3152 & 0.99 \\
\hline 187 & 188.0 & 0.72 & 0.1270 & 0.3381 & 0.99 \\
\hline 188 & 192.2 & 0.57 & 0.2199 & 0.2642 & 1.00 \\
\hline 189 & 196.0 & 0.53 & 0.2718 & 0.2729 & 0.99 \\
\hline 190 & 200.1 & 0.58 & 0.3471 & 0.3189 & 0.98 \\
\hline 191 & 204.2 & 0.65 & 0.4600 & 0.3571 & 1.00 \\
\hline 192 & 208.1 & 0.78 & 0.6256 & 0.4231 & 0.96 \\
\hline 193 & 211.9 & 0.98 & 0.8752 & 0.5024 & 0.93 \\
\hline
\end{tabular}




\begin{tabular}{|c|c|c|c|c|c|}
\hline \multicolumn{6}{|c|}{ Table B1. LS(1)-0417MOD, Clean, $R e=1$ million } \\
\hline RUN & $\mathrm{AOA}$ & $\mathrm{C}_{1}$ & $\mathrm{C}_{\mathrm{dp}}$ & $\mathrm{C}_{\mathrm{m} / 4}$ & $\operatorname{Re} \times 10^{-6}$ \\
\hline 194 & 216.0 & 0.94 & 0.9417 & 0.5156 & 0.89 \\
\hline 195 & 219.8 & 0.99 & 1.1053 & 0.5599 & 0.82 \\
\hline 196 & 223.8 & 1.05 & 1.4040 & 0.6100 & 0.79 \\
\hline 197 & 227.9 & 0.99 & 1.4705 & 0.6286 & 0.77 \\
\hline 198 & 231.0 & 0.96 & 1.5822 & 0.6286 & 0.71 \\
\hline \multicolumn{6}{|c|}{ End of Table B1 } \\
\hline
\end{tabular}




\begin{tabular}{|c|c|c|c|c|c|}
\hline \multicolumn{6}{|c|}{ Table B2. LS(1)-0417MOD, Clean, $\mathrm{Re}=1.5$ million } \\
\hline RUN & $\mathrm{AOA}$ & $\mathrm{C}_{1}$ & $\mathrm{C}_{\mathrm{dp}}$ & $\mathrm{C}_{\mathrm{m} / 4}$ & $\operatorname{Re} \times 10^{-6}$ \\
\hline 201 & -25.8 & -0.52 & 0.3684 & 0.0480 & 1.45 \\
\hline 202 & -22.0 & -0.40 & 0.2735 & 0.0271 & 1.47 \\
\hline 203 & -19.9 & -0.36 & 0.2441 & 0.0220 & 1.49 \\
\hline 204 & -18.0 & -0.35 & 0.2228 & 0.0263 & 1.50 \\
\hline 205 & -16.0 & -0.29 & 0.1922 & 0.0200 & 1.51 \\
\hline 206 & -14.1 & -0.27 & 0.1672 & 0.0176 & 1.48 \\
\hline 207 & -12.0 & -0.74 & 0.0387 & -0.0569 & 1.49 \\
\hline 208 & -9.7 & -0.54 & 0.0246 & -0.0646 & 1.49 \\
\hline 209 & -8.0 & -0.35 & 0.0182 & -0.0667 & 1.49 \\
\hline 210 & -6.0 & -0.15 & 0.0081 & -0.0695 & 1.49 \\
\hline 211 & -3.9 & 0.05 & 0.0014 & -0.0705 & 1.48 \\
\hline 212 & -1.8 & 0.27 & -.0030 & -0.0772 & 1.49 \\
\hline 213 & -0.1 & 0.46 & -.0052 & -0.0799 & 1.49 \\
\hline 214 & 2.0 & 0.69 & -.0064 & -0.0833 & 1.49 \\
\hline 215 & 4.0 & 0.89 & -.0038 & -0.0819 & 1.49 \\
\hline 216 & 6.1 & 1.09 & -.0013 & -0.0808 & 1.48 \\
\hline 217 & 8.2 & 1.27 & 0.0038 & -0.0779 & 1.48 \\
\hline 218 & 10.3 & 1.44 & 0.0073 & -0.0720 & 1.48 \\
\hline 219 & 10.9 & 1.48 & 0.0111 & -0.0697 & 1.49 \\
\hline 220 & 12.0 & 1.54 & 0.0144 & -0.0653 & 1.49 \\
\hline 221 & 13.0 & 1.58 & 0.0193 & -0.0623 & 1.49 \\
\hline 222 & 14.1 & 1.51 & 0.0760 & -0.0817 & 1.49 \\
\hline 223 & 15.1 & 1.55 & 0.1003 & -0.0925 & 1.49 \\
\hline 224 & 15.9 & 1.59 & 0.1209 & -0.1026 & 1.49 \\
\hline 225 & 16.9 & 1.26 & 0.1353 & -0.1076 & 1.49 \\
\hline 226 & 17.9 & 1.07 & 0.1614 & -0.1250 & 1.49 \\
\hline 227 & 19.0 & 1.46 & 0.1870 & -0.1071 & 1.49 \\
\hline
\end{tabular}




\begin{tabular}{||c|c|c|c|c|c||}
\hline \multicolumn{7}{|c|}{ Table B2. LS(1)-0417MOD, Clean, Re $=1.5$ million } \\
\hline \hline RUN & AOA & $\mathrm{C}_{1}$ & $\mathrm{C}_{\mathrm{dp}}$ & $\mathrm{C}_{\mathrm{m} / \mathrm{s}}$ & ${\operatorname{Re~} \mathrm{x} 10^{-6}}^{-6}$ \\
\hline 228 & 20.0 & 1.44 & 0.2014 & -0.1031 & 1.50 \\
\hline 229 & 22.1 & 1.38 & 0.2380 & -0.1217 & 1.49 \\
\hline 230 & 24.1 & 1.28 & 0.2897 & -0.1465 & 1.50 \\
\hline 231 & 26.1 & 1.07 & 0.3358 & -0.1578 & 1.49 \\
\hline 232 & 27.9 & 1.06 & 0.3677 & -0.1623 & 1.49 \\
\hline 233 & 30.0 & 1.10 & 0.4473 & -0.1865 & 1.50 \\
\hline 234 & 32.1 & 0.91 & 0.5984 & -0.1914 & 1.49 \\
\hline 235 & 34.1 & 0.92 & 0.6488 & -0.1985 & 1.48 \\
\hline 236 & 36.0 & 0.94 & 0.7066 & -0.2108 & 1.49 \\
\hline 237 & 38.0 & 0.93 & 0.7503 & -0.2176 & 1.48 \\
\hline 238 & 40.1 & 1.17 & 1.0041 & -0.2939 & 1.42 \\
\hline & & End of Table B2 & & \\
\hline \hline
\end{tabular}




\begin{tabular}{|c|c|c|c|c|c|}
\hline \multicolumn{6}{|c|}{ Table B3. LS(1)-0417MOD, Clean, $\mathrm{Re}=2$ million } \\
\hline RUN & $\mathrm{AOA}$ & $\mathrm{C}_{1}$ & $\mathrm{C}_{\mathrm{dp}}$ & $\mathrm{C}_{\mathrm{m} / \mathrm{s}}$ & $\operatorname{Re} \times 10^{-6}$ \\
\hline 250 & -20.1 & -0.42 & 0.2703 & 0.0353 & 2.16 \\
\hline 251 & -18.0 & -0.40 & 0.2411 & 0.0277 & 2.16 \\
\hline 252 & -16.0 & -0.35 & 0.2087 & 0.0290 & 1.98 \\
\hline 253 & -13.9 & -0.45 & 0.1906 & 0.0271 & 1.99 \\
\hline 254 & -12.3 & -0.79 & 0.0362 & -0.0608 & 2.02 \\
\hline 255 & -10.1 & -0.57 & 0.0265 & -0.0639 & 2.02 \\
\hline 256 & -9.0 & -0.48 & 0.0207 & -0.0658 & 2.02 \\
\hline 257 & -8.2 & -0.41 & 0.0164 & -0.0667 & 2.02 \\
\hline 258 & -7.0 & -0.26 & 0.0131 & -0.0677 & 2.01 \\
\hline 259 & -6.0 & -0.17 & 0.0088 & -0.0674 & 2.01 \\
\hline 260 & -5.0 & -0.07 & 0.0047 & -0.0691 & 2.01 \\
\hline 261 & -3.9 & 0.05 & 0.0016 & -0.0709 & 2.02 \\
\hline 262 & -1.9 & 0.26 & -.0027 & -0.0770 & 2.02 \\
\hline 263 & -0.1 & 0.46 & -.0051 & -0.0805 & 2.01 \\
\hline 264 & 2.0 & 0.69 & -.0058 & -0.0830 & 2.01 \\
\hline 265 & 4.1 & 0.89 & -.0038 & -0.0832 & 2.02 \\
\hline 266 & 6.1 & 1.12 & -.0063 & -0.0818 & 2.01 \\
\hline 267 & 8.2 & 1.27 & 0.0069 & -0.0791 & 2.09 \\
\hline 268 & 10.2 & 1.46 & 0.0080 & -0.0746 & 2.09 \\
\hline 269 & 11.2 & 1.52 & 0.0116 & -0.0706 & 2.08 \\
\hline 270 & 12.3 & 1.58 & 0.0162 & -0.0658 & 2.06 \\
\hline 271 & 13.0 & 1.63 & 0.0176 & -0.0640 & 2.05 \\
\hline 272 & 14.0 & 1.39 & 0.1034 & -0.1124 & 2.04 \\
\hline 273 & 15.4 & 1.35 & 0.1261 & -0.1143 & 2.04 \\
\hline 274 & 15.9 & 1.30 & 0.1252 & -0.1063 & 2.04 \\
\hline 275 & 16.9 & 1.07 & 0.1511 & -0.1251 & 2.02 \\
\hline 276 & 18.0 & 1.11 & 0.1670 & -0.1241 & 2.01 \\
\hline
\end{tabular}




\begin{tabular}{|c|c|c|c|c|c||}
\hline \multicolumn{7}{|c|}{ Table B3. LS(1)-0417MOD, Clean, Re = 2 million } \\
\hline \hline RUN & AOA & $\mathrm{C}_{1}$ & $\mathrm{C}_{\mathrm{dp}}$ & $\mathrm{C}_{\mathrm{m} / 4}$ & $\mathrm{Re}_{10} 0^{-6}$ \\
\hline 277 & 19.0 & 1.43 & 0.1938 & -0.1110 & 1.99 \\
\hline 278 & 20.0 & 1.41 & 0.2125 & -0.1119 & 1.99 \\
\hline 279 & 22.0 & 1.29 & 0.2428 & -0.1273 & 1.96 \\
\hline 280 & 24.1 & 1.20 & 0.2964 & -0.1471 & 1.95 \\
\hline 281 & 26.1 & 1.03 & 0.3240 & -0.1455 & 1.93 \\
\hline 282 & 28.0 & 1.04 & 0.3788 & -0.1614 & 1.92 \\
\hline 283 & 30.0 & 1.05 & 0.4441 & -0.1790 & 1.90 \\
\hline 284 & 32.1 & 0.94 & 0.6192 & -0.2073 & 1.87 \\
\hline 285 & 33.9 & 0.90 & 0.6328 & -0.1947 & 1.85 \\
\hline 286 & 36.0 & 0.96 & 0.7159 & -0.2154 & 1.83 \\
\hline 287 & 38.0 & 0.96 & 0.7720 & -0.2254 & 1.80 \\
\hline 288 & 40.1 & 0.94 & 0.8094 & -0.2278 & 1.77 \\
\hline & & End of Table B3 & & \\
\hline
\end{tabular}




\begin{tabular}{|c|c|c|c|c|c|}
\hline \multicolumn{6}{|c|}{ Table B4. LS(1)-0417MOD, LEGR $k / c=0.0019, R e=1$ million } \\
\hline RUN & $\mathrm{AOA}$ & $\mathrm{C}_{1}$ & $\mathrm{C}_{\mathrm{dp}}$ & $\mathrm{C}_{\mathrm{m} / \mathrm{A}}$ & $\operatorname{Re} \times 10^{-6}$ \\
\hline 290 & -59.6 & -1.05 & 1.7466 & 0.4086 & 0.75 \\
\hline 291 & -57.9 & -1.02 & 1.5992 & 0.3634 & 0.76 \\
\hline 292 & -53.8 & -1.05 & 1.4305 & 0.3142 & 0.77 \\
\hline 293 & -49.9 & -1.08 & 1.2987 & 0.2823 & 0.79 \\
\hline 294 & -46.1 & -1.16 & 1.2496 & 0.2833 & 0.81 \\
\hline 295 & -41.9 & -1.08 & 1.0347 & 0.2302 & 0.83 \\
\hline 296 & -37.8 & -0.96 & 0.8190 & 0.1649 & 0.89 \\
\hline 297 & -33.9 & -0.86 & 0.6740 & 0.1325 & 0.92 \\
\hline 298 & -30.1 & -0.71 & 0.5178 & 0.0910 & 1.01 \\
\hline 299 & -26.0 & -0.65 & 0.4270 & 0.0752 & 0.99 \\
\hline 300 & -22.1 & -0.47 & 0.2986 & 0.0416 & 1.00 \\
\hline 301 & -20.0 & -0.42 & 0.2516 & 0.0239 & 1.00 \\
\hline 302 & -18.0 & -0.50 & 0.2290 & 0.0284 & 1.01 \\
\hline 303 & -15.9 & -0.54 & 0.1888 & 0.0242 & 1.02 \\
\hline 304 & -14.1 & -0.60 & 0.1185 & -0.0128 & 1.00 \\
\hline 305 & -12.1 & -0.61 & 0.0496 & -0.0601 & 1.00 \\
\hline 306 & -10.0 & -0.51 & 0.0330 & -0.0593 & 1.00 \\
\hline 307 & -8.0 & -0.35 & 0.0209 & -0.0583 & 1.00 \\
\hline 308 & -5.8 & -0.15 & 0.0107 & -0.0596 & 1.00 \\
\hline 309 & -4.0 & 0.01 & 0.0032 & -0.0614 & 0.99 \\
\hline 310 & -2.0 & 0.23 & -.0030 & -0.0647 & 0.99 \\
\hline 311 & 0.0 & 0.42 & -.0066 & -0.0652 & 0.99 \\
\hline 312 & 2.1 & 0.64 & -.0079 & -0.0669 & 1.00 \\
\hline 313 & 4.2 & 0.82 & -.0062 & -0.0642 & 0.99 \\
\hline 314 & 5.9 & 0.97 & -.0061 & -0.0604 & 1.00 \\
\hline 315 & 8.0 & 1.09 & 0.0027 & -0.0550 & 0.99 \\
\hline 316 & 10.0 & 1.12 & 0.0423 & -0.0627 & 0.99 \\
\hline
\end{tabular}




\begin{tabular}{|c|c|c|c|c|c|}
\hline \multicolumn{6}{|c|}{ Table B4. LS(1)-0417MOD, LEGR $\mathrm{k} / \mathrm{c}=0.0019, \mathrm{Re}=1$ million } \\
\hline RUN & AOA & $\mathrm{C}_{\mathrm{l}}$ & $\mathrm{C}_{\mathrm{dp}}$ & $\mathrm{C}_{\mathrm{m} / 4}$ & $\operatorname{Re} \times 10^{-6}$ \\
\hline 317 & 11.1 & 0.99 & 0.1001 & -0.0990 & 1.00 \\
\hline 318 & 12.1 & 0.95 & 0.1135 & -0.0992 & 0.99 \\
\hline 319 & 13.1 & 0.93 & 0.1282 & -0.1000 & 0.99 \\
\hline 320 & 14.0 & 0.90 & 0.1387 & -0.0966 & 0.99 \\
\hline 321 & 15.0 & 0.90 & 0.1595 & -0.1052 & 0.99 \\
\hline 322 & 16.0 & 0.89 & 0.1735 & -0.1038 & 0.99 \\
\hline 323 & 17.1 & 0.85 & 0.1932 & -0.1064 & 1.00 \\
\hline 324 & 18.1 & 0.83 & 0.2130 & -0.1120 & 1.00 \\
\hline 325 & 19.1 & 0.81 & 0.2372 & -0.1158 & 0.99 \\
\hline 326 & 20.1 & 0.83 & 0.2714 & -0.1276 & 0.99 \\
\hline 327 & 22.2 & 0.92 & 0.3374 & -0.1495 & 0.98 \\
\hline 328 & 24.0 & 0.96 & 0.3949 & -0.1674 & 1.00 \\
\hline 329 & 26.0 & 1.00 & 0.4647 & -0.1860 & 0.99 \\
\hline 330 & 28.1 & 1.03 & 0.5225 & -0.1960 & 1.00 \\
\hline 331 & 30.1 & 1.05 & 0.5983 & -0.2144 & 0.98 \\
\hline 332 & 32.0 & 1.21 & 0.7398 & -0.2656 & 1.00 \\
\hline 333 & 34.0 & 1.28 & 0.8424 & -0.2928 & 0.98 \\
\hline 334 & 36.1 & 1.32 & 0.9321 & -0.3105 & 0.99 \\
\hline 335 & 38.1 & 1.33 & 1.0180 & -0.3287 & 1.00 \\
\hline 336 & 39.9 & 1.31 & 1.0671 & -0.3301 & 0.99 \\
\hline 337 & 44.0 & 1.36 & 1.2757 & -0.3761 & 0.95 \\
\hline 338 & 48.2 & 1.28 & 1.3897 & -0.3945 & 0.91 \\
\hline 339 & 52.0 & 1.37 & 1.7165 & -0.4839 & 0.88 \\
\hline 340 & 55.9 & 1.29 & 1.8241 & -0.4970 & 0.85 \\
\hline 341 & 60.0 & 1.18 & 1.9439 & -0.5176 & 0.82 \\
\hline 342 & 64.2 & 1.07 & 2.0845 & -0.5487 & 0.81 \\
\hline 343 & 68.0 & 0.98 & 2.2775 & -0.6065 & 0.78 \\
\hline
\end{tabular}




\begin{tabular}{|c|c|c|c|c|c|}
\hline \multicolumn{6}{|c|}{ Table B4. LS(1)-0417MOD, LEGR $\mathrm{k} / \mathrm{c}=0.0019, \mathrm{Re}=1$ million } \\
\hline RUN & $\mathrm{AOA}$ & $\mathrm{C}_{1}$ & $C_{d p}$ & $\mathrm{C}_{\mathrm{m} / \mathrm{A}}$ & $\operatorname{Re} \times 10^{-6}$ \\
\hline 344 & 72.2 & 0.80 & 2.2386 & -0.5867 & 0.75 \\
\hline 345 & 76.0 & 0.72 & 2.4993 & -0.6482 & 0.75 \\
\hline 346 & 80.2 & 0.53 & 2.4017 & -0.6347 & 0.72 \\
\hline 347 & 84.0 & 0.38 & 2.5666 & -0.6890 & 0.71 \\
\hline 348 & 88.2 & 0.18 & 2.3121 & -0.6356 & 0.69 \\
\hline 349 & 92.0 & 0.05 & 2.4472 & -0.6727 & 0.70 \\
\hline 350 & 96.1 & -0.11 & 2.3864 & -0.6828 & 0.70 \\
\hline 351 & 100.0 & -0.25 & 2.2996 & -0.6745 & 0.70 \\
\hline 352 & 104.0 & -0.43 & 2.3265 & -0.7078 & 0.68 \\
\hline 353 & 108.2 & -0.58 & 2.2918 & -0.7203 & 0.67 \\
\hline 354 & 111.8 & -0.67 & 2.1689 & -0.6975 & 0.68 \\
\hline 355 & 115.9 & -0.76 & 1.9872 & -0.6744 & 0.69 \\
\hline 356 & 120.1 & -0.89 & 1.9050 & -0.6818 & 0.69 \\
\hline 357 & 124.2 & -0.89 & 1.5996 & -0.6267 & 0.71 \\
\hline 358 & 128.1 & -0.97 & 1.5307 & -0.6196 & 0.73 \\
\hline 359 & 132.1 & -1.04 & 1.3922 & -0.6122 & 0.75 \\
\hline 360 & 136.0 & -1.14 & 1.3455 & -0.6187 & 0.76 \\
\hline 361 & 140.1 & -1.06 & 1.1082 & -0.5455 & 0.79 \\
\hline 362 & 144.1 & -0.93 & 0.8575 & -0.5040 & 0.80 \\
\hline 363 & 148.1 & -0.85 & 0.6661 & -0.4560 & 0.91 \\
\hline 364 & 152.2 & -0.65 & 0.4492 & -0.3695 & 0.95 \\
\hline 365 & 156.2 & -0.55 & 0.3453 & -0.3227 & 1.00 \\
\hline 366 & 160.1 & -0.59 & 0.2956 & -0.3127 & 0.98 \\
\hline 367 & 164.1 & -0.70 & 0.2149 & -0.3424 & 1.00 \\
\hline 368 & 168.2 & -0.85 & 0.1130 & -0.4109 & 1.00 \\
\hline 369 & 172.0 & -0.57 & 0.0577 & -0.2502 & 1.01 \\
\hline 370 & 176.1 & -0.19 & 0.0223 & -0.0864 & 1.01 \\
\hline
\end{tabular}




\begin{tabular}{|c|c|c|c|c|c||}
\hline \multicolumn{7}{|c|}{ Table B4. LS(1)-0417MOD, LEGR k/c=0.0019, Re = 1 million } \\
\hline \hline RUN & AOA & $\mathrm{C}_{1}$ & $\mathrm{C}_{\mathrm{dp}}$ & $\mathrm{C}_{\mathrm{m} / \mathrm{s}}$ & $\operatorname{Re} \times 10^{-6}$ \\
\hline 371 & 180.0 & 0.28 & 0.0305 & 0.1104 & 1.01 \\
\hline 372 & 184.3 & 0.65 & 0.0523 & 0.3159 & 1.01 \\
\hline 373 & 187.9 & 0.70 & 0.1229 & 0.3298 & 1.00 \\
\hline 374 & 192.1 & 0.56 & 0.2121 & 0.2679 & 1.00 \\
\hline 375 & 196.1 & 0.49 & 0.2611 & 0.2668 & 0.98 \\
\hline 376 & 200.1 & 0.55 & 0.3391 & 0.3071 & 1.00 \\
\hline 377 & 203.9 & 0.62 & 0.4492 & 0.3554 & 0.99 \\
\hline 378 & 208.0 & 0.50 & 0.4972 & 0.3897 & 1.00 \\
\hline 379 & 212.1 & 0.59 & 0.6449 & 0.4511 & 0.97 \\
\hline 380 & 216.1 & 0.70 & 0.8337 & 0.5105 & 0.93 \\
\hline 381 & 219.9 & 0.80 & 1.0344 & 0.5592 & 0.90 \\
\hline 382 & 224.0 & 0.81 & 1.1933 & 0.5951 & 0.88 \\
\hline 383 & 228.1 & 0.73 & 1.2522 & 0.5950 & 0.85 \\
\hline 384 & 231.2 & 0.79 & 1.4616 & 0.6397 & 0.83 \\
\hline & & End of Table B4 & & \\
\hline \hline
\end{tabular}




\begin{tabular}{|c|c|c|c|c|c|}
\hline \multicolumn{6}{|c|}{ Table B5. LS(1)-0417MOD, LEGR $\mathrm{k} / \mathrm{c}=0.0019, \mathrm{Re}=1.5$ million } \\
\hline RUN & $\mathrm{AOA}$ & $\mathrm{C}_{1}$ & $\mathrm{C}_{\mathrm{dp}}$ & $\mathrm{C}_{\mathrm{m}^{3 / 4}}$ & $\operatorname{Re} \times 10^{-6}$ \\
\hline 400 & -29.9 & -0.75 & 0.5281 & 0.0967 & 1.37 \\
\hline 401 & -25.8 & -0.58 & 0.3890 & 0.0575 & 1.41 \\
\hline 402 & -22.0 & -0.43 & 0.2840 & 0.0252 & 1.49 \\
\hline 403 & -19.9 & -0.45 & 0.2499 & 0.0207 & 1.50 \\
\hline 404 & -18.0 & -0.54 & 0.2345 & 0.0333 & 1.50 \\
\hline 405 & -16.0 & -0.67 & 0.1417 & -0.0181 & 1.49 \\
\hline 406 & -13.9 & -0.65 & 0.1118 & -0.0150 & 1.48 \\
\hline 407 & -11.9 & -0.63 & 0.0476 & -0.0613 & 1.49 \\
\hline 408 & -9.9 & -0.49 & 0.0326 & -0.0601 & 1.50 \\
\hline 409 & -8.0 & -0.36 & 0.0208 & -0.0598 & 1.50 \\
\hline 410 & -6.0 & -0.16 & 0.0116 & -0.0602 & 1.50 \\
\hline 411 & -4.0 & 0.05 & 0.0033 & -0.0638 & 1.50 \\
\hline 412 & -1.9 & 0.26 & -.0025 & -0.0654 & 1.50 \\
\hline 413 & 0.1 & 0.46 & -.0068 & -0.0675 & $\cdot 1.50$ \\
\hline 414 & 2.0 & 0.65 & -.0086 & -0.0682 & 1.50 \\
\hline 415 & 4.0 & 0.83 & -.0048 & -0.0673 & 1.49 \\
\hline 416 & 6.1 & 0.98 & 0.0003 & -0.0627 & 1.49 \\
\hline 417 & 8.2 & 1.14 & 0.0044 & -0.0575 & 1.49 \\
\hline 418 & 10.2 & 0.86 & 0.0857 & -0.1003 & 1.50 \\
\hline 419 & 11.0 & 0.86 & 0.0974 & -0.0987 & 1.48 \\
\hline 420 & 12.0 & 0.93 & 0.1148 & -0.0992 & 1.48 \\
\hline 421 & 13.1 & 0.90 & 0.1286 & -0.0967 & 1.49 \\
\hline 422 & 14.0 & 0.90 & 0.1411 & -0.0977 & 1.50 \\
\hline 423 & 15.0 & 0.87 & 0.1579 & -0.1011 & 1.49 \\
\hline 424 & 16.1 & 0.84 & 0.1760 & -0.1022 & 1.49 \\
\hline 425 & 16.9 & 0.80 & 0.1937 & -0.1077 & 1.49 \\
\hline 426 & 17.9 & 0.82 & 0.2193 & -0.1160 & 1.48 \\
\hline
\end{tabular}




\begin{tabular}{||c|c|c|c|c|c||}
\hline \multicolumn{6}{|c||}{ Table B5. LS(1)-0417MOD, LEGR k/c=0.0019, Re = 1.5 million } \\
\hline \hline RUN & AOA & $\mathrm{C}_{\mathrm{l}}$ & $\mathrm{C}_{\mathrm{dp}}$ & $\mathrm{C}_{\mathrm{m} / /}$ & $\operatorname{Re} \times 10^{-6}$ \\
\hline 427 & 19.0 & 0.85 & 0.2502 & -0.1262 & 1.50 \\
\hline 428 & 20.0 & 0.85 & 0.2754 & -0.1316 & 1.50 \\
\hline 429 & 22.1 & 0.94 & 0.3521 & -0.1606 & 1.48 \\
\hline 430 & 24.1 & 0.94 & 0.3891 & -0.1624 & 1.52 \\
\hline 431 & 26.1 & 1.00 & 0.4640 & -0.1847 & 1.50 \\
\hline 432 & 28.0 & 1.08 & 0.5477 & -0.2118 & 1.50 \\
\hline 433 & 30.0 & 1.18 & 0.6526 & -0.2439 & 1.49 \\
\hline 434 & 32.1 & 1.29 & 0.7849 & -0.2838 & 1.48 \\
\hline 435 & 34.3 & 1.30 & 0.8587 & -0.2945 & 1.45 \\
\hline 436 & 36.0 & 1.44 & 1.0090 & -0.3466 & 1.38 \\
\hline \multicolumn{6}{|c|}{ End of Table B5 } \\
\hline
\end{tabular}




\begin{tabular}{|c|c|c|c|c|c|}
\hline \multicolumn{6}{|c|}{ Table B6. LS(1)-0417MOD, LEGR $\mathrm{k} / \mathrm{c}=0.0019, \mathrm{Re}=2$ million } \\
\hline RUN & $\mathrm{AOA}$ & $\mathrm{C}_{\mathrm{l}}$ & $\mathrm{C}_{\mathrm{dp}}$ & $\mathrm{C}_{\mathrm{m} / \mathrm{s}}$ & $\operatorname{Re} \times 10^{-6}$ \\
\hline 440 & -20.1 & -0.43 & 0.2607 & 0.0240 & 1.88 \\
\hline 441 & -18.0 & -0.47 & 0.2254 & 0.0196 & 1.87 \\
\hline 442 & -16.0 & -0.64 & 0.1760 & 0.0080 & 1.88 \\
\hline 443 & -13.9 & -0.63 & 0.1309 & -0.0023 & 1.91 \\
\hline 444 & -11.9 & -0.65 & 0.0481 & -0.0616 & 1.99 \\
\hline 445 & -10.1 & -0.55 & 0.0361 & -0.0612 & 1.99 \\
\hline 446 & -8.0 & -0.38 & 0.0239 & -0.0600 & 1.99 \\
\hline 447 & -6.0 & -0.20 & 0.0123 & -0.0611 & 1.99 \\
\hline 448 & -3.9 & 0.02 & 0.0036 & -0.0631 & 1.99 \\
\hline 449 & -1.8 & 0.24 & -.0020 & -0.0655 & 1.99 \\
\hline 450 & 0.2 & 0.46 & -.0063 & -0.0681 & 1.99 \\
\hline 451 & 2.0 & 0.65 & -.0077 & -0.0683 & 1.99 \\
\hline 452 & 4.1 & 0.84 & -.0065 & -0.0674 & 1.98 \\
\hline 453 & 6.1 & 1.02 & -.0032 & -0.0641 & 1.98 \\
\hline 454 & 8.2 & 1.16 & 0.0028 & -0.0588 & 1.97 \\
\hline 455 & 9.9 & 0.80 & 0.0737 & -0.0825 & 1.99 \\
\hline 456 & 11.0 & 0.83 & 0.1001 & -0.0969 & 1.98 \\
\hline 457 & 12.0 & 0.94 & 0.1082 & -0.0895 & 1.97 \\
\hline 458 & 13.0 & 0.94 & 0.1307 & -0.1010 & 1.99 \\
\hline 459 & 14.1 & 0.85 & 0.1464 & -0.1005 & 1.98 \\
\hline 460 & 15.1 & 0.85 & 0.1643 & -0.1035 & 2.00 \\
\hline 461 & 16.1 & 0.81 & 0.1809 & -0.1047 & 1.99 \\
\hline 462 & 17.2 & 0.82 & 0.2038 & -0.1121 & 1.98 \\
\hline 463 & 18.0 & 0.83 & 0.2252 & -0.1180 & 1.97 \\
\hline 464 & 19.0 & 0.84 & 0.2593 & -0.1303 & 1.99 \\
\hline 465 & 20.0 & 0.86 & 0.2902 & -0.1390 & 1.98 \\
\hline 466 & 22.1 & 0.93 & 0.3530 & -0.1602 & 1.99 \\
\hline
\end{tabular}




\begin{tabular}{|c|c|c|c|c|c||}
\hline \multicolumn{7}{|c|}{ Table B6. LS(1)-0417MOD, LEGR k/c=0.0019, Re =2 million } \\
\hline \hline RUN & AOA & $\mathrm{C}_{1}$ & $\mathrm{C}_{\mathrm{dp}}$ & $\mathrm{C}_{\mathrm{m} / 4}$ & ${\operatorname{Re~} \times 10^{-6}}$ \\
\hline 467 & 24.1 & 0.97 & 0.4140 & -0.1740 & 1.99 \\
\hline 468 & 26.2 & 0.99 & 0.4766 & -0.1879 & 1.97 \\
\hline 469 & 28.3 & 1.05 & 0.5642 & -0.2122 & 1.93 \\
\hline 470 & 30.3 & 1.13 & 0.6518 & -0.2352 & 1.87 \\
\hline 471 & 32.1 & 1.22 & 0.7512 & -0.2657 & 1.82 \\
\hline \multicolumn{7}{|c|}{ End of Table B6 } \\
\hline
\end{tabular}




\begin{tabular}{|c|c|c|c|c|c|}
\hline \multicolumn{6}{|c|}{ Table B7. LS(1)-0417MOD, LEGR k/c=0.0019, VGs, $R e=1$ million } \\
\hline RUN & $\mathrm{AOA}$ & $\mathrm{C}_{1}$ & $C_{d p}$ & $\mathrm{C}_{\mathrm{m} / \mathrm{t}}$ & $\operatorname{Re} \times 10^{-6}$ \\
\hline 480 & -20.0 & -0.38 & 0.2492 & 0.0170 & 0.98 \\
\hline 481 & -17.9 & -0.49 & 0.2522 & 0.0351 & 0.98 \\
\hline 482 & -16.1 & -0.63 & 0.1822 & -0.0041 & 1.00 \\
\hline 483 & -14.0 & -0.60 & 0.1392 & -0.0046 & 1.00 \\
\hline 484 & -11.9 & -0.63 & 0.0506 & -0.0567 & 1.01 \\
\hline 485 & -10.1 & -0.54 & 0.0350 & -0.0606 & 1.01 \\
\hline 486 & -8.0 & -0.40 & 0.0223 & -0.0604 & 1.01 \\
\hline 487 & -5.9 & -0.23 & 0.0134 & -0.0618 & 1.01 \\
\hline 488 & -4.1 & -0.06 & 0.0068 & -0.0630 & 0.99 \\
\hline 489 & -2.0 & 0.16 & 0.0031 & -0.0670 & 1.00 \\
\hline 490 & 0.1 & 0.37 & -.0002 & -0.0704 & 1.00 \\
\hline 491 & 2.0 & 0.59 & -.0003 & -0.0746 & 1.00 \\
\hline 492 & 4.0 & 0.78 & 0.0028 & -0.0739 & 1.00 \\
\hline 493 & 5.9 & 0.99 & 0.0058 & -0.0767 & 1.00 \\
\hline 494 & 7.9 & 1.16 & 0.0125 & -0.0776 & 1.00 \\
\hline 495 & 10.0 & 1.34 & 0.0249 & -0.0788 & 0.99 \\
\hline 496 & 11.1 & 1.41 & 0.0333 & -0.0776 & 0.99 \\
\hline 497 & 12.1 & 0.91 & 0.1124 & -0.0997 & 1.00 \\
\hline 498 & 12.9 & 0.88 & 0.1198 & -0.0939 & 1.00 \\
\hline 499 & 13.9 & 0.86 & 0.1415 & -0.0999 & 0.99 \\
\hline 500 & 15.0 & 0.85 & 0.1550 & -0.0996 & 0.99 \\
\hline 501 & 16.0 & 0.78 & 0.1661 & -0.0966 & 0.99 \\
\hline 502 & 17.1 & 0.80 & 0.1905 & -0.1050 & 0.99 \\
\hline 503 & 18.1 & 0.82 & 0.2090 & -0.1065 & 0.99 \\
\hline 504 & 19.1 & 0.83 & 0.2349 & -0.1158 & 0.99 \\
\hline 505 & 19.9 & 0.82 & 0.2548 & -0.1200 & 1.00 \\
\hline 506 & 22.0 & 0.85 & 0.3132 & -0.1373 & 0.99 \\
\hline
\end{tabular}




\begin{tabular}{|c|c|c|c|c|c||}
\hline \multicolumn{6}{|c|}{ Table B7. LS(1)-0417MOD, LEGR k/c=0.0019, VGs, Re =1 million } \\
\hline \hline RUN & AOA & $\mathrm{C}_{1}$ & $\mathrm{C}_{\mathrm{dp}}$ & $\mathrm{C}_{\mathrm{m} / \%}$ & $\operatorname{Re} \times 10^{-6}$ \\
\hline 507 & 23.9 & 0.94 & 0.3928 & -0.1660 & 1.00 \\
\hline 508 & 26.0 & 0.97 & 0.4515 & -0.1797 & 0.99 \\
\hline 509 & 28.0 & 1.02 & 0.5314 & -0.1992 & 0.99 \\
\hline 510 & 30.1 & 1.07 & 0.6155 & -0.2214 & 1.00 \\
\hline 511 & 31.9 & 1.14 & 0.7001 & -0.2419 & 1.00 \\
\hline 512 & 34.0 & 1.22 & 0.8117 & -0.2745 & 0.98 \\
\hline 513 & 36.1 & 1.32 & 0.9493 & -0.3180 & 0.99 \\
\hline 514 & 38.0 & 1.28 & 0.9780 & -0.3110 & 0.98 \\
\hline 515 & 40.0 & 1.30 & 1.0665 & -0.3307 & 1.00 \\
\hline \multicolumn{7}{|c|}{ End of Table B7 } \\
\hline
\end{tabular}




\begin{tabular}{|c|c|c|c|c|c|}
\hline \multicolumn{6}{|c|}{ Table B8. LS(1)-0417MOD, LEGR k/c=0.0019, VGs, $\mathrm{Re}=1.5$ million } \\
\hline RUN & $\mathrm{AOA}$ & $\mathrm{C}_{1}$ & $C_{d p}$ & $\mathrm{C}_{\mathrm{m} / \mathrm{h}}$ & $\operatorname{Re} \times 10^{-6}$ \\
\hline 520 & -20.0 & -0.38 & 0.2466 & 0.0129 & 1.49 \\
\hline 521 & -17.9 & -0.53 & 0.2294 & 0.0160 & 1.50 \\
\hline 522 & -16.1 & -0.52 & 0.2043 & 0.0165 & 1.51 \\
\hline 523 & -14.0 & -0.66 & 0.1285 & -0.0108 & 1.48 \\
\hline 524 & -11.9 & -0.65 & 0.0494 & -0.0606 & 1.50 \\
\hline 525 & -10.1 & -0.54 & 0.0361 & -0.0608 & 1.50 \\
\hline 526 & -8.0 & -0.39 & 0.0232 & -0.0610 & 1.50 \\
\hline 527 & -5.9 & -0.20 & 0.0126 & -0.0635 & 1.49 \\
\hline 528 & -3.8 & -0.01 & 0.0051 & -0.0658 & 1.50 \\
\hline 529 & -2.0 & 0.18 & 0.0016 & -0.0690 & 1.50 \\
\hline 530 & 0.1 & 0.39 & -.0006 & -0.0716 & 1.50 \\
\hline 531 & 2.0 & 0.59 & -.0016 & -0.0740 & 1.49 \\
\hline 532 & 4.0 & 0.80 & 0.0012 & -0.0749 & 1.49 \\
\hline 533 & 6.1 & 0.98 & 0.0078 & -0.0759 & 1.49 \\
\hline 534 & 7.9 & 1.16 & 0.0128 & -0.0772 & 1.49 \\
\hline 535 & 10.0 & 1.32 & 0.0260 & -0.0761 & 1.49 \\
\hline 536 & 11.1 & 1.40 & 0.0360 & -0.0768 & 1.49 \\
\hline 537 & 12.1 & 0.76 & 0.1051 & -0.0849 & 1.47 \\
\hline 538 & 12.9 & 0.79 & 0.1236 & -0.0970 & 1.48 \\
\hline 539 & 13.9 & 0.88 & 0.1452 & -0.1015 & 1.50 \\
\hline 540 & 14.9 & 0.81 & 0.1595 & -0.0996 & 1.49 \\
\hline 541 & 16.0 & 0.78 & 0.1718 & -0.0977 & 1.48 \\
\hline 542 & 17.1 & 0.77 & 0.1929 & -0.1028 & 1.48 \\
\hline 543 & 17.9 & 0.81 & 0.2120 & -0.1082 & 1.50 \\
\hline 544 & 18.9 & 0.80 & 0.2337 & -0.1137 & 1.50 \\
\hline 545 & 19.9 & 0.84 & 0.2713 & -0.1295 & 1.49 \\
\hline 546 & 22.0 & 0.89 & 0.3405 & -0.1527 & 1.49 \\
\hline
\end{tabular}




\begin{tabular}{|c|c|c|c|c|c||}
\hline \multicolumn{6}{|c|}{ Table B8. LS(1)-0417MOD, LEGR k/c=0.0019, VGs, Re = 1.5 million } \\
\hline \hline RUN & $\mathrm{AOA}$ & $\mathrm{C}_{1}$ & $\mathrm{C}_{\mathrm{dp}}$ & $\mathrm{C}_{\mathrm{m} /}$ & $\operatorname{Re} \times 10^{-6}$ \\
\hline 547 & 24.1 & 0.90 & 0.3897 & -0.1598 & 1.47 \\
\hline 548 & 25.9 & 0.97 & 0.4613 & -0.1817 & 1.50 \\
\hline 549 & 28.0 & 1.03 & 0.5387 & -0.2021 & 1.50 \\
\hline 550 & 29.8 & 1.11 & 0.6272 & -0.2281 & 1.50 \\
\hline 551 & 32.0 & 1.16 & 0.7145 & -0.2492 & 1.47 \\
\hline 552 & 34.0 & 1.22 & 0.8159 & -0.2764 & 1.50 \\
\hline 553 & 35.9 & 1.31 & 0.9361 & -0.3142 & 1.48 \\
\hline & \multicolumn{5}{|c}{ End of Table B8 } \\
\hline
\end{tabular}




\begin{tabular}{|c|c|c|c|c|c|}
\hline \multicolumn{6}{|c|}{ Table B9. LS(1)-0417MOD, Clean, VGs, $\mathrm{Re}=1$ million } \\
\hline RUN & $\mathrm{AOA}$ & $\mathrm{C}_{1}$ & $\mathrm{C}_{\mathrm{dp}}$ & $\mathrm{C}_{\mathrm{m}^{1 / 4}}$ & $\operatorname{Re} \times 10^{-6}$ \\
\hline 560 & -20.0 & -0.38 & 0.2613 & 0.0179 & 1.00 \\
\hline 561 & -17.9 & -0.29 & 0.2130 & 0.0032 & 1.00 \\
\hline 562 & -16.0 & -0.29 & 0.2005 & 0.0076 & 1.00 \\
\hline 563 & -14.1 & -0.24 & 0.1700 & -0.0007 & 1.01 \\
\hline 564 & -12.1 & -0.71 & 0.0396 & -0.0603 & 1.00 \\
\hline 565 & -10.0 & -0.60 & 0.0283 & -0.0585 & 1.00 \\
\hline 566 & -7.9 & -0.45 & 0.0212 & -0.0590 & 1.00 \\
\hline 567 & -6.0 & -0.29 & 0.0107 & -0.0642 & 1.00 \\
\hline 568 & -4.0 & -0.07 & 0.0059 & -0.0696 & 1.01 \\
\hline 569 & -2.0 & 0.12 & 0.0038 & -0.0755 & 1.00 \\
\hline 570 & 0.1 & 0.36 & 0.0022 & -0.0817 & 0.99 \\
\hline 571 & 2.0 & 0.58 & 0.0043 & -0.0852 & 1.00 \\
\hline 572 & 3.9 & 0.77 & 0.0099 & -0.0846 & 1.00 \\
\hline 573 & 6.0 & 0.97 & 0.0160 & -0.0869 & 0.99 \\
\hline 574 & 8.1 & 1.16 & 0.0263 & -0.0892 & 1.00 \\
\hline 575 & 9.9 & 1.34 & 0.0364 & -0.0895 & 0.99 \\
\hline 576 & 10.9 & 1.44 & 0.0418 & -0.0903 & 0.99 \\
\hline 577 & 12.0 & 1.51 & 0.0523 & -0.0903 & 0.99 \\
\hline 578 & 13.0 & 1.62 & 0.0542 & -0.0901 & 0.99 \\
\hline 579 & 14.1 & 1.70 & 0.0616 & -0.0900 & 0.99 \\
\hline 580 & 14.9 & 1.78 & 0.0708 & -0.0913 & 0.99 \\
\hline 581 & 15.9 & 1.84 & 0.0766 & -0.0876 & 0.99 \\
\hline 582 & 17.0 & 1.88 & 0.0906 & -0.0858 & 1.00 \\
\hline 583 & 18.0 & 1.11 & 0.1460 & -0.1180 & 0.99 \\
\hline 584 & 19.1 & 1.06 & 0.1548 & -0.1111 & 0.99 \\
\hline 585 & 19.9 & 1.56 & 0.2020 & -0.0887 & 0.99 \\
\hline 586 & 22.0 & 1.48 & 0.2470 & -0.1178 & 0.99 \\
\hline
\end{tabular}




\begin{tabular}{||c|c|c|c|c|c||}
\hline \multicolumn{7}{|c|}{ Table B9. LS(1)-0417MOD, Clean, VGs, Re = 1 million } \\
\hline \hline RUN & AOA & $\mathrm{C}_{1}$ & $\mathrm{C}_{\mathrm{dp}}$ & $\mathrm{C}_{\mathrm{m} / 4}$ & $\operatorname{Re} \times 10^{-5}$ \\
\hline 587 & 24.1 & 1.37 & 0.2803 & -0.1313 & 1.00 \\
\hline 588 & 25.9 & 1.18 & 0.3032 & -0.1403 & 1.00 \\
\hline 589 & 28.0 & 1.08 & 0.3472 & -0.1519 & 1.00 \\
\hline 590 & 30.1 & 0.87 & 0.5399 & -0.1798 & 1.00 \\
\hline 591 & 32.0 & 0.91 & 0.5896 & -0.1867 & 1.00 \\
\hline 592 & 34.0 & 0.94 & 0.6638 & -0.2040 & 1.01 \\
\hline 593 & 36.1 & 1.01 & 0.7595 & -0.2280 & 0.99 \\
\hline 594 & 37.9 & 1.28 & 1.0274 & -0.3186 & 1.00 \\
\hline 595 & 40.0 & 1.35 & 1.1498 & -0.3446 & 1.00 \\
\hline & & End of Table B9 & & \\
\hline \hline
\end{tabular}




\begin{tabular}{|c|c|c|c|c|c|}
\hline \multicolumn{6}{|c|}{ Table B10. LS(1)-0417MOD, Clean, VGs, $R e=1.5$ million } \\
\hline RUN & $\mathrm{AOA}$ & $\mathrm{C}_{1}$ & $\mathrm{C}_{\mathrm{dp}}$ & $\mathrm{C}_{\mathrm{m}^{1 / 4}}$ & $\operatorname{Re} \times 10^{-6}$ \\
\hline 600 & -20.0 & -0.38 & 0.2518 & 0.0144 & 1.50 \\
\hline 601 & -17.9 & -0.32 & 0.2169 & 0.0065 & 1.48 \\
\hline 602 & -16.0 & -0.28 & 0.1921 & 0.0035 & 1.49 \\
\hline 603 & -13.9 & -0.26 & 0.1740 & 0.0081 & 1.50 \\
\hline 604 & -12.1 & -0.77 & 0.0335 & -0.0612 & 1.50 \\
\hline 605 & -10.0 & -0.63 & 0.0253 & -0.0600 & 1.51 \\
\hline 606 & -7.9 & -0.49 & 0.0155 & -0.0601 & 1.49 \\
\hline 607 & -6.0 & -0.30 & 0.0106 & -0.0635 & 1.49 \\
\hline 608 & -3.9 & -0.05 & 0.0048 & -0.0705 & 1.50 \\
\hline 609 & -1.8 & 0.16 & 0.0026 & -0.0768 & 1.50 \\
\hline 610 & -0.0 & 0.36 & 0.0021 & -0.0818 & 1.50 \\
\hline 611 & 2.0 & 0.59 & 0.0042 & -0.0858 & 1.50 \\
\hline 612 & 3.9 & 0.80 & 0.0054 & -0.0884 & 1.50 \\
\hline 613 & 6.0 & 1.01 & 0.0125 & -0.0904 & 1.49 \\
\hline 614 & 8.1 & 1.19 & 0.0230 & -0.0914 & 1.50 \\
\hline 615 & 9.9 & 1.38 & 0.0288 & -0.0914 & 1.49 \\
\hline 616 & 11.0 & 1.45 & 0.0398 & -0.0916 & 1.49 \\
\hline 617 & 11.8 & 1.53 & 0.0421 & -0.0909 & 1.49 \\
\hline 618 & 13.1 & 1.64 & 0.0509 & -0.0899 & 1.49 \\
\hline 619 & 14.1 & 1.72 & 0.0593 & -0.0892 & 1.48 \\
\hline 620 & 14.9 & 1.77 & 0.0684 & -0.0890 & 1.50 \\
\hline 621 & 15.9 & 1.85 & 0.0765 & -0.0890 & 1.49 \\
\hline 622 & 17.0 & 1.09 & 0.1409 & -0.1230 & 1.48 \\
\hline 623 & 18.0 & 1.05 & 0.1550 & -0.1200 & 1.50 \\
\hline 624 & 19.1 & 1.45 & 0.1923 & -0.1009 & 1.49 \\
\hline 625 & 20.1 & 1.41 & 0.2092 & -0.1062 & 1.49 \\
\hline 626 & 22.2 & 1.41 & 0.2511 & -0.1170 & 1.50 \\
\hline
\end{tabular}




\begin{tabular}{|c|c|c|c|c|c||}
\hline \multicolumn{7}{|c|}{ Table B10. LS(1)-0417MOD, Clean, VGs, Re = 1.5 million } \\
\hline \hline RUN & AOA & $\mathrm{C}_{1}$ & $\mathrm{C}_{\mathrm{dp}}$ & $\mathrm{C}_{\mathrm{m} /}$ & $\operatorname{Re}_{10} 0^{-6}$ \\
\hline 627 & 24.2 & 1.26 & 0.2798 & -0.1315 & 1.49 \\
\hline 628 & 25.9 & 1.03 & 0.3189 & -0.1507 & 1.51 \\
\hline 629 & 28.0 & 1.04 & 0.3637 & -0.1573 & 1.50 \\
\hline 630 & 30.1 & 1.05 & 0.4174 & -0.1693 & 1.48 \\
\hline 631 & 32.0 & 1.08 & 0.4848 & -0.1893 & 1.50 \\
\hline 632 & 34.0 & 1.09 & 0.5365 & -0.1998 & 1.49 \\
\hline 633 & 35.8 & 0.89 & 0.6706 & -0.1982 & 1.50 \\
\hline 634 & 37.9 & 0.93 & 0.7423 & -0.2123 & 1.51 \\
\hline 635 & 40.0 & 1.03 & 0.8851 & -0.2511 & 1.49 \\
\hline & & \multicolumn{6}{|c|}{ End of Table B10 } & & \\
\hline
\end{tabular}




\begin{tabular}{|c|c|c|c|c|c|}
\hline \multicolumn{6}{|c|}{ Table B11. LS(1)-0417MOD, Clean, $\operatorname{Re}=1$ million, Small $\alpha$ Cal Range } \\
\hline RUN & $\mathrm{AOA}$ & $\mathrm{C}_{1}$ & $\mathrm{C}_{\mathrm{dp}}$ & $\mathrm{C}_{\mathrm{m} / \mathrm{s}}$ & $\operatorname{Re} \times 10^{-6}$ \\
\hline 640 & -20.1 & -0.37 & 0.2521 & 0.0272 & 1.00 \\
\hline 641 & -18.0 & -0.39 & 0.2385 & 0.0344 & 1.00 \\
\hline 642 & -16.0 & -0.27 & 0.1852 & 0.0136 & 1.01 \\
\hline 643 & -14.0 & -0.29 & 0.1762 & 0.0281 & 1.00 \\
\hline 644 & -11.9 & -0.74 & 0.0428 & -0.0463 & 1.01 \\
\hline 645 & -10.0 & -0.56 & 0.0299 & -0.0608 & 1.00 \\
\hline 646 & -7.9 & -0.38 & 0.0194 & -0.0647 & 1.00 \\
\hline 647 & -6.0 & -0.21 & 0.0105 & -0.0703 & 1.00 \\
\hline 648 & -4.0 & -0.03 & 0.0045 & -0.0712 & 1.00 \\
\hline 649 & -2.1 & 0.19 & 0.0013 & -0.0752 & 1.00 \\
\hline 650 & -0.0 & 0.40 & -.0013 & -0.0794 & 1.01 \\
\hline 651 & 2.1 & 0.61 & 0.0016 & -0.0801 & 1.01 \\
\hline 652 & 4.0 & 0.80 & 0.0077 & -0.0796 & 1.00 \\
\hline 653 & 6.1 & 1.00 & 0.0108 & -0.0784 & -1.00 \\
\hline 654 & 8.0 & 1.17 & 0.0185 & -0.0779 & 1.00 \\
\hline 655 & 10.0 & 1.32 & 0.0277 & -0.0724 & 0.99 \\
\hline 656 & 11.1 & 1.39 & 0.0346 & -0.0700 & 1.00 \\
\hline 657 & 11.9 & 1.45 & 0.0390 & -0.0677 & 1.00 \\
\hline 658 & 13.0 & 1.52 & 0.0447 & -0.0638 & 1.00 \\
\hline 659 & 14.0 & 1.56 & 0.0484 & -0.0586 & 0.99 \\
\hline 660 & 15.1 & 1.55 & 0.0835 & -0.0662 & 0.99 \\
\hline 661 & 16.1 & 1.57 & 0.1018 & -0.0671 & 0.99 \\
\hline 662 & 17.2 & 1.06 & 0.1252 & -0.1070 & 1.00 \\
\hline 663 & 17.9 & 1.06 & 0.1357 & -0.1080 & 1.00 \\
\hline 664 & 19.0 & 1.51 & 0.1812 & -0.0938 & 0.99 \\
\hline 665 & 20.1 & 1.48 & 0.2048 & -0.1003 & 1.00 \\
\hline 666 & 21.9 & 1.42 & 0.2352 & -0.1148 & 1.00 \\
\hline
\end{tabular}




\begin{tabular}{|c|c|c|c|c|c||}
\hline \multicolumn{6}{|c|}{ Table B11. LS(1)-0417MOD, Clean, Re $=1$ million, Small $\alpha$ Cal Range } \\
\hline \hline RUN & AOA & $\mathrm{C}_{1}$ & $\mathrm{C}_{\mathrm{dp}}$ & $\mathrm{C}_{\mathrm{m} / 4}$ & $\operatorname{Re} \times 10^{-6}$ \\
\hline 667 & 24.0 & 1.35 & 0.2731 & -0.1273 & 1.00 \\
\hline 668 & 26.1 & 1.13 & 0.3073 & -0.1429 & 0.99 \\
\hline 669 & 28.0 & 1.04 & 0.3392 & -0.1471 & 0.99 \\
\hline 670 & 30.0 & 1.15 & 0.4181 & -0.1686 & 1.00 \\
\hline 671 & 32.0 & 1.16 & 0.4784 & -0.1882 & 0.99 \\
\hline 672 & 34.0 & 1.19 & 0.8212 & -0.2671 & 1.00 \\
\hline 673 & 35.9 & 1.20 & 0.8904 & -0.2793 & 0.99 \\
\hline 674 & 38.0 & 1.31 & 1.0460 & -0.3280 & 1.00 \\
\hline 675 & 40.1 & 1.30 & 1.1211 & -0.3425 & 0.99 \\
\hline & & End of Table B11 & & \\
\hline \hline
\end{tabular}




\begin{tabular}{|c|c|c|c|c|c|}
\hline RUN & $\mathrm{AOA}$ & $\mathrm{C}_{1}$ & $\mathrm{C}_{\mathrm{dp}}$ & $\mathrm{C}_{\mathrm{m} / \mathrm{s}}$ & $\operatorname{Re} \times 10^{-6}$ \\
\hline 680 & -20.1 & -0.37 & 0.2435 & 0.0220 & 1.50 \\
\hline 681 & -18.0 & -0.37 & 0.2291 & 0.0255 & 1.50 \\
\hline 682 & -15.9 & -0.31 & 0.1953 & 0.0206 & 1.49 \\
\hline 683 & -14.0 & -0.29 & 0.1749 & 0.0203 & 1.49 \\
\hline 684 & -11.9 & -0.76 & 0.0358 & -0.0543 & 1.49 \\
\hline 685 & -10.0 & -0.60 & 0.0237 & -0.0639 & 1.49 \\
\hline 686 & -7.9 & -0.40 & 0.0163 & -0.0669 & 1.49 \\
\hline 687 & -6.1 & -0.22 & 0.0092 & -0.0690 & 1.49 \\
\hline 688 & -4.0 & -0.01 & 0.0033 & -0.0698 & 1.49 \\
\hline 689 & -1.9 & 0.20 & 0.0004 & -0.0747 & 1.50 \\
\hline 690 & -0.0 & 0.40 & -.0015 & -0.0782 & 1.49 \\
\hline 691 & 2.1 & 0.61 & 0.0019 & -0.0814 & 1.49 \\
\hline 692 & 4.0 & 0.79 & 0.0066 & -0.0819 & 1.49 \\
\hline 693 & 6.1 & 0.98 & 0.0121 & -0.0785 & 1.49 \\
\hline 694 & 7.9 & 1.17 & 0.0171 & -0.0779 & 1.49 \\
\hline 695 & 10.0 & 1.33 & 0.0259 & -0.0740 & 1.49 \\
\hline 696 & 11.2 & 1.42 & 0.0344 & -0.0708 & 1.50 \\
\hline 697 & 12.1 & 1.46 & 0.0439 & -0.0690 & 1.50 \\
\hline 698 & 13.1 & 1.52 & 0.0492 & -0.0655 & 1.49 \\
\hline 699 & 14.0 & 1.57 & 0.0530 & -0.0612 & 1.49 \\
\hline 700 & 15.0 & 1.60 & 0.0610 & -0.0576 & 1.49 \\
\hline 701 & 15.8 & 1.08 & 0.1154 & -0.1118 & 1.50 \\
\hline 702 & 17.1 & 1.05 & 0.1326 & -0.1107 & 1.49 \\
\hline 703 & 17.9 & 1.01 & 0.1468 & -0.1128 & 1.50 \\
\hline 704 & 19.0 & 1.09 & 0.1766 & -0.1234 & 1.49 \\
\hline 705 & 20.0 & 1.43 & 0.2137 & -0.1127 & 1.49 \\
\hline 706 & 22.1 & 1.39 & 0.2467 & -0.1168 & 1.50 \\
\hline
\end{tabular}




\begin{tabular}{||c|c|c|c|c|c||}
\hline \multicolumn{5}{|c|}{ Table B12. LS(1)-0417MOD, Clean, $\mathrm{Re}=1.5$ million, Small $\alpha$ Cal Range } \\
\hline \hline RUN & $\mathrm{AOA}$ & $\mathrm{C}_{\mathrm{l}}$ & $\mathrm{C}_{\mathrm{dp}}$ & $\mathrm{C}_{\mathrm{m} / 4}$ & ${\operatorname{Re~} 10^{-6}}$ \\
\hline 707 & 24.0 & 1.32 & 0.2779 & -0.1265 & 1.49 \\
\hline 708 & 26.1 & 1.17 & 0.3386 & -0.1542 & 1.49 \\
\hline 709 & 28.2 & 1.02 & 0.3585 & -0.1499 & 1.50 \\
\hline 710 & 30.0 & 1.06 & 0.4127 & -0.1656 & 1.49 \\
\hline 711 & 31.6 & 1.10 & 0.4793 & -0.1855 & 1.50 \\
\hline 712 & 34.1 & 0.88 & 0.6208 & -0.1877 & 1.48 \\
\hline 713 & 36.1 & 0.90 & 0.6814 & -0.2000 & 1.50 \\
\hline 714 & 38.0 & 0.97 & 0.7719 & -0.2229 & 1.49 \\
\hline 715 & 40.1 & 1.11 & 0.9667 & -0.2812 & 1.50 \\
\hline & & \multicolumn{6}{|c|}{ End of Table B12 } & & \\
\hline
\end{tabular}


Steady State Pressure Distributions

$$
\operatorname{Re}=1.0 \text { million }
$$


$C_{p}$ VERSUS $x / c$

NASA LS(1) -0417 MOD $R e=1 . \times 10^{6}$

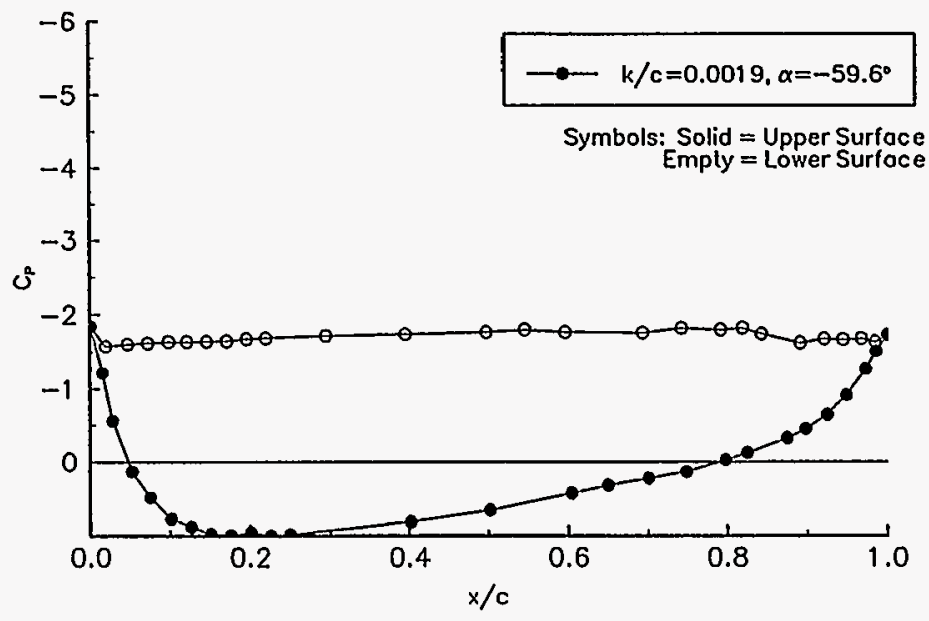

Figure 1. $\alpha=-60^{\circ}$

$C_{p}$ VERSUS $x / c$

NASA LS(1) $-0417 \mathrm{MOO} R e=1 . \times 10^{6}$

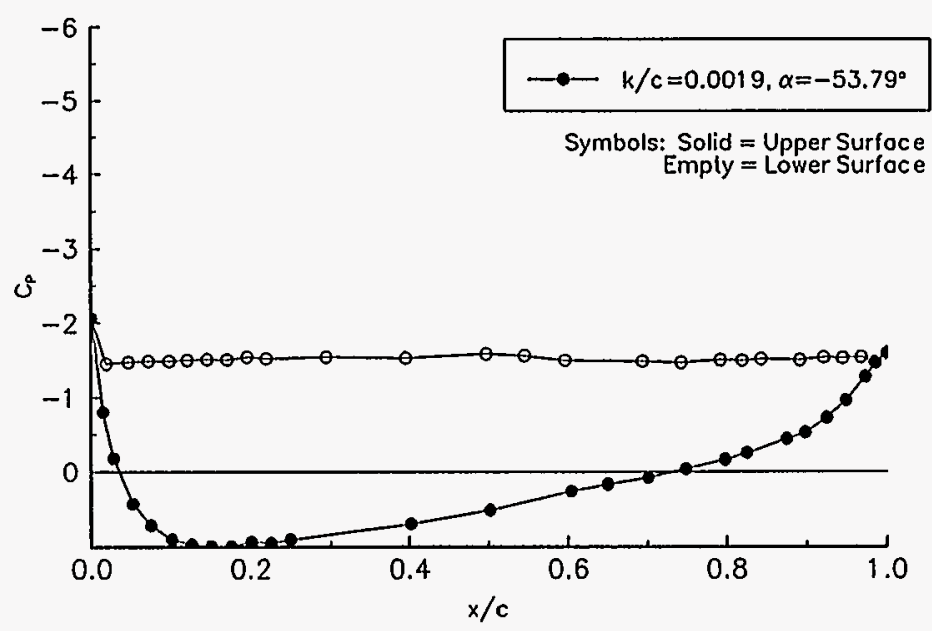

Figure 3. $\alpha=-54^{\circ}$
$C_{p}$ VERSUS $x / c$

NASA LS(1) -0417 MOD $R e=1 . \times 10^{\circ}$

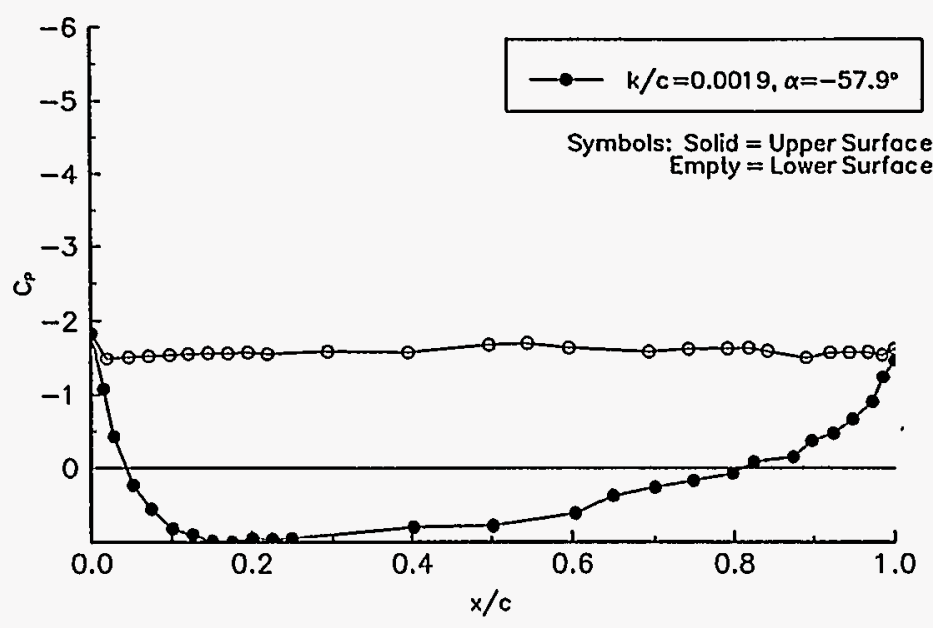

Figure 2. $\alpha=-58^{\circ}$

$C_{p}$ VERSUS $x / c$

NASA LS (1) -0417 MOD Re $=1 . \times 10^{6}$

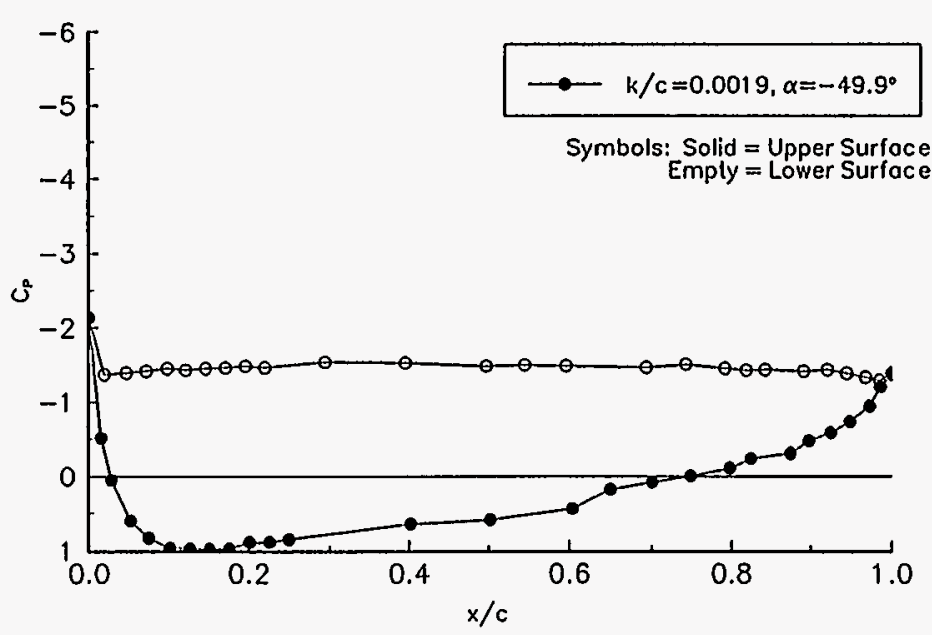

Figure 4. $\alpha=-50^{\circ}$ 
$C_{p}$ VERSUS $x / c$

NASA LS(1) -0417 MOD $R e=1 . \times 10^{\circ}$

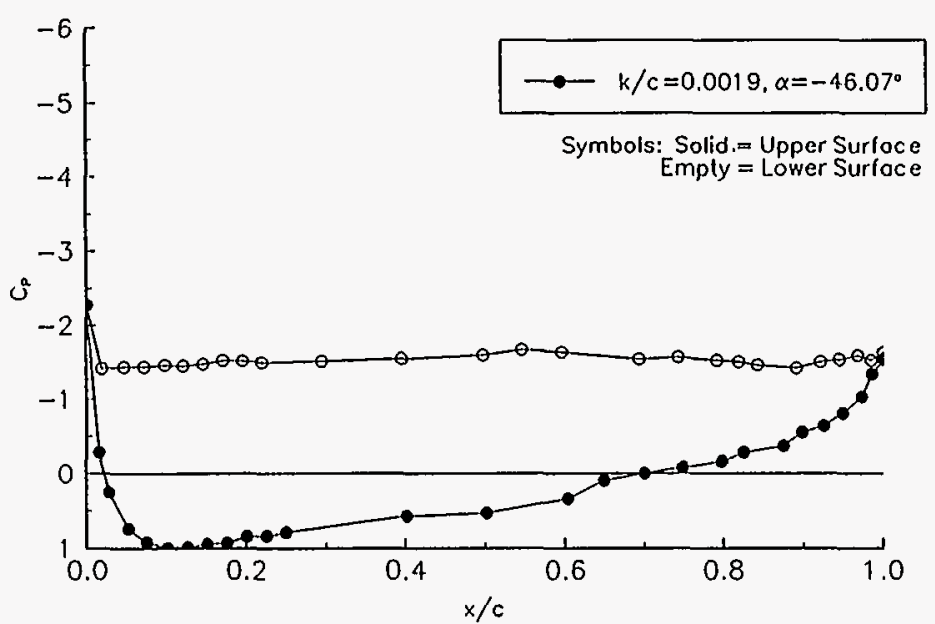

Figure 5. $\alpha=-46^{\circ}$

$C_{P}$ VERSUS $\times /$

NASA LS(1) -0417 MOD Re $=1 . \times 10^{\circ}$

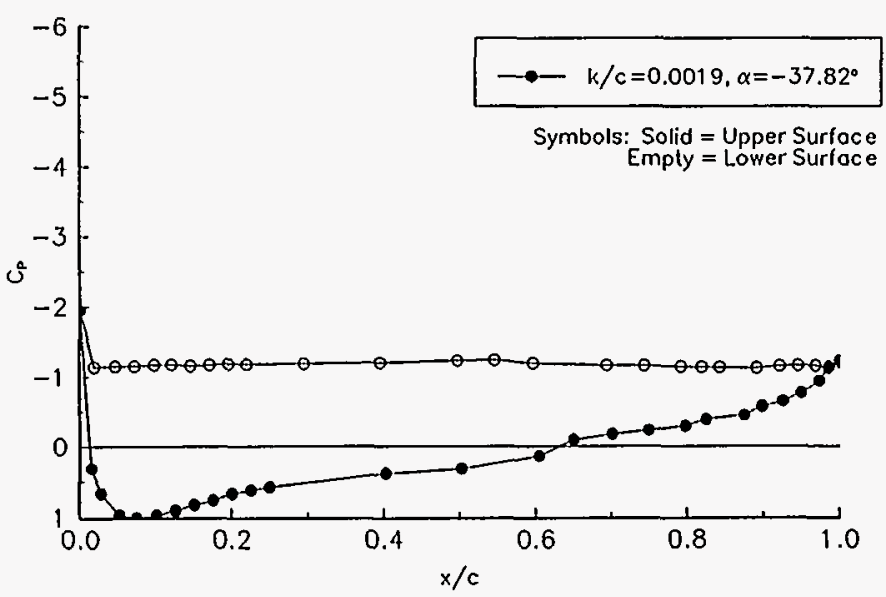

Figure 7. $\alpha=-38^{\circ}$
$C_{p}$ VERSUS $x / c$

NASA LS $(1)-0417$ MOD $R e=1 . \times 10^{\circ}$

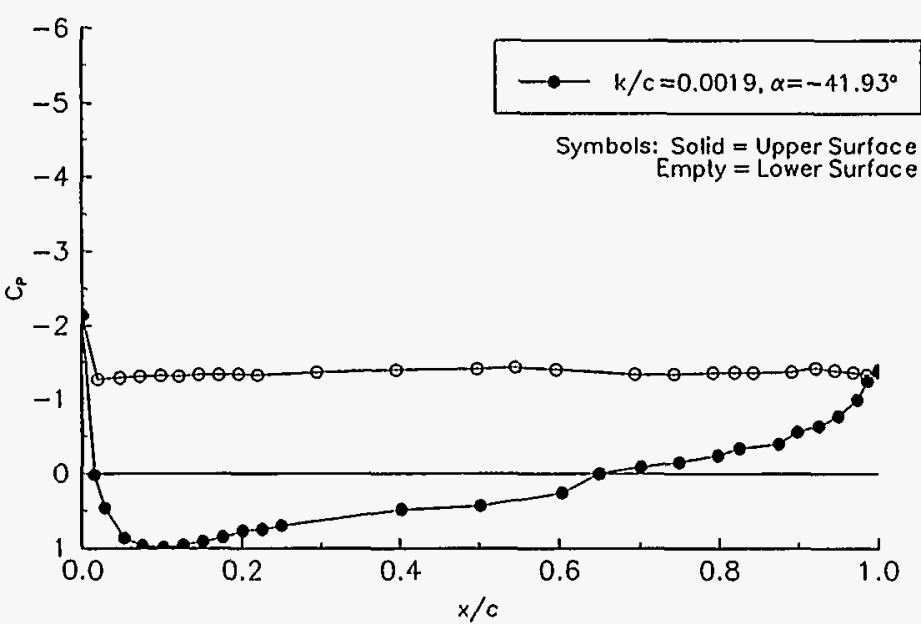

Figure 6. $\alpha=-42^{\circ}$

$C_{0}$ VERSUS $x / c$

NASA LS(1) -0417 MOD $R e=1 . \times 10^{\circ}$

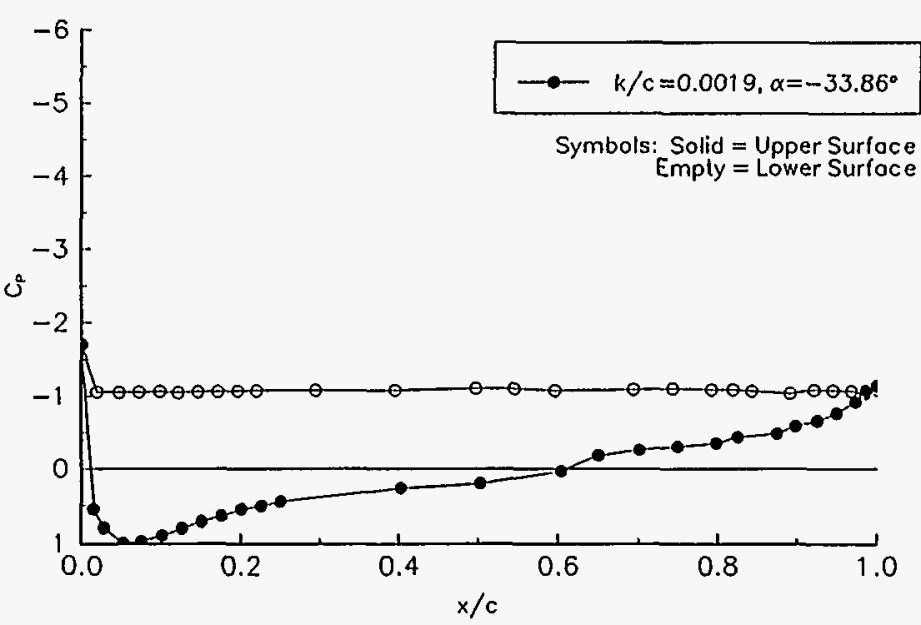

Figure 8. $\alpha=-34^{\circ}$ 


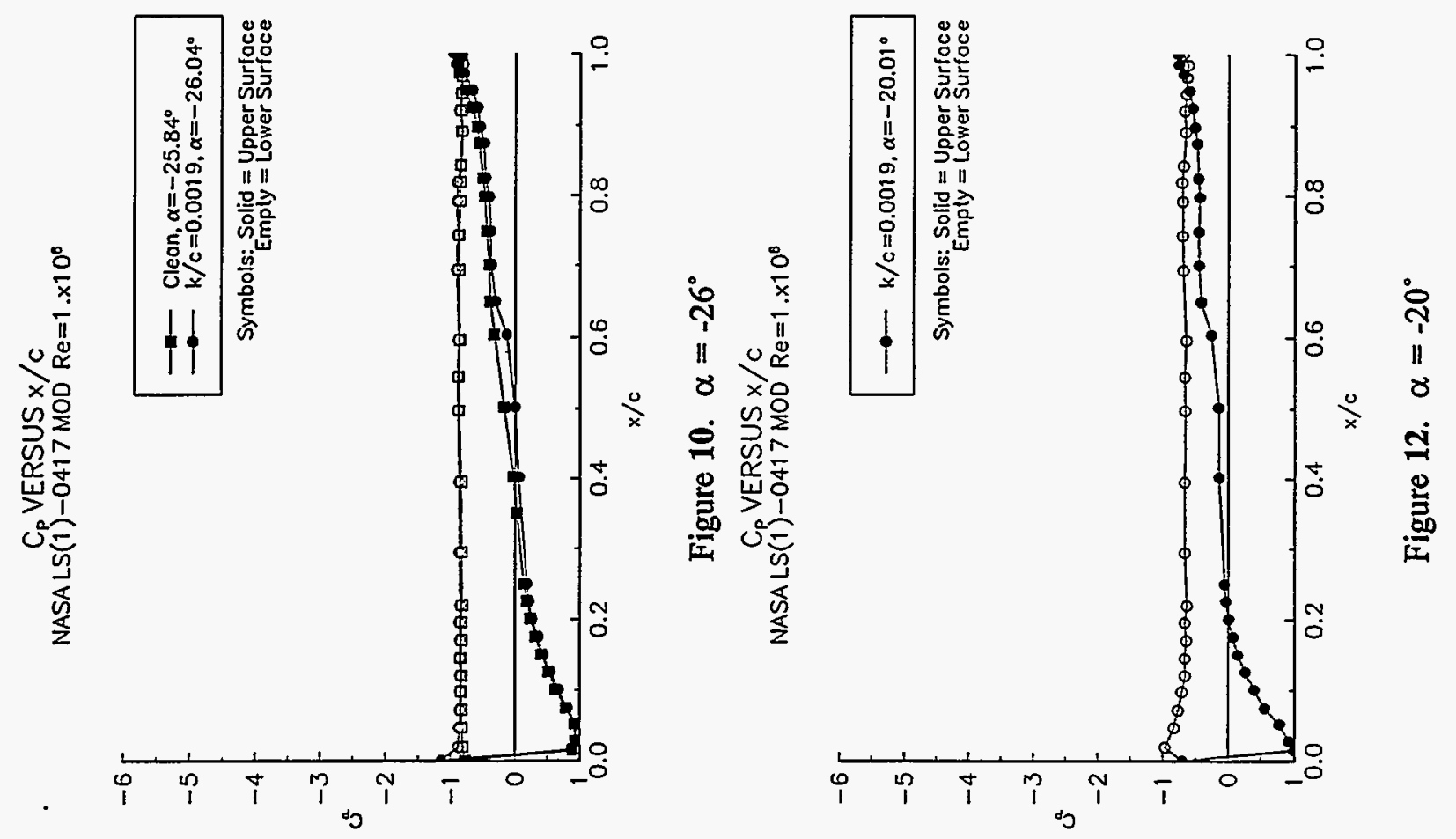

ติ

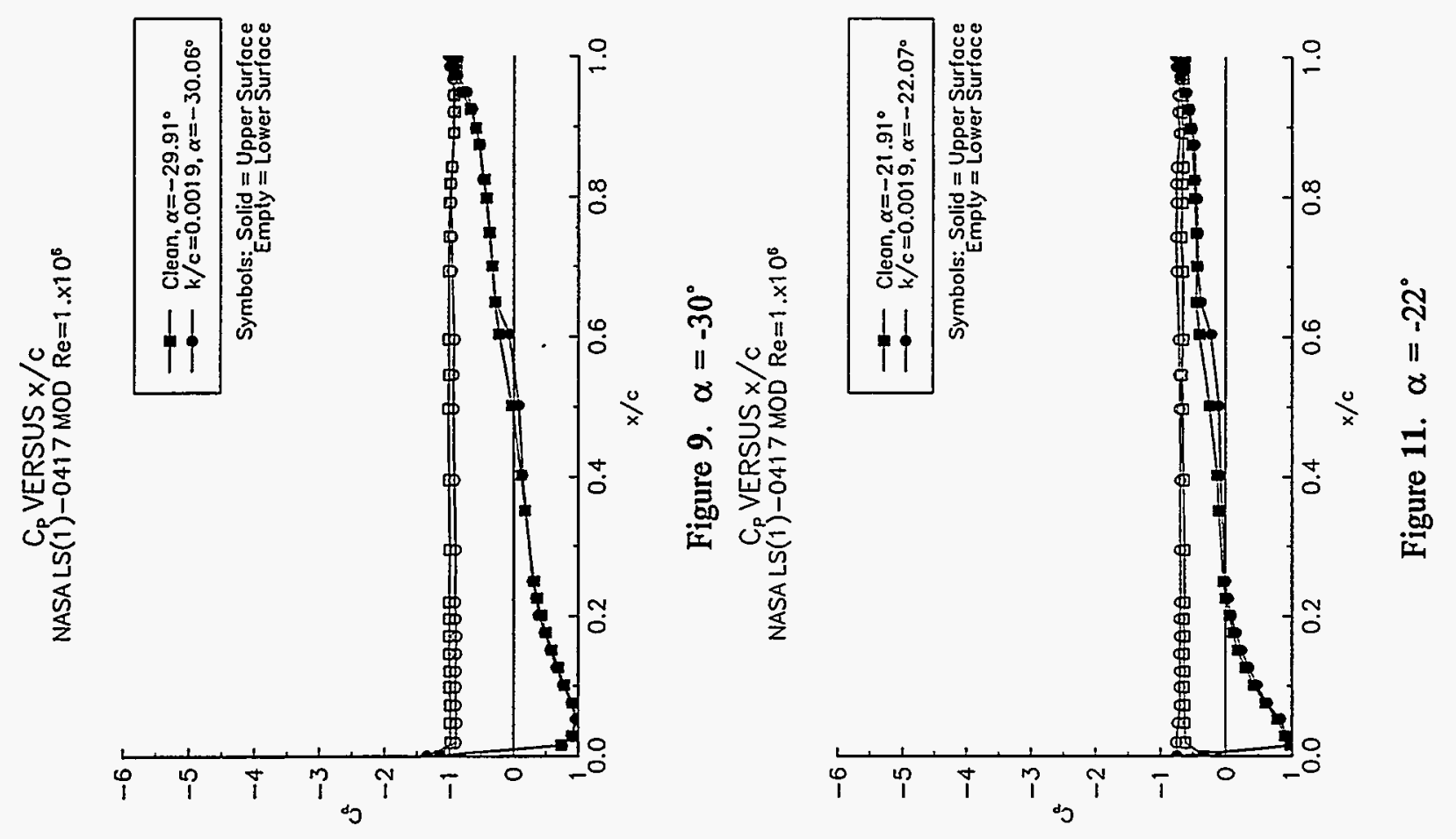


$C_{p}$ VERSUS $x / c$

NASA LS(1)-0417 MOD Re $=1 . \times 10^{\circ}$

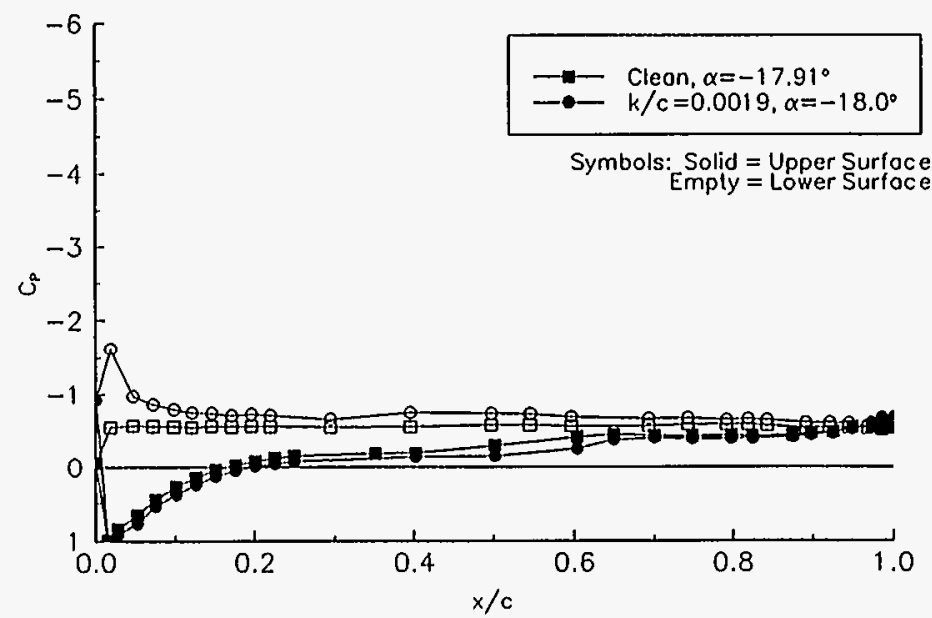

Figure 13. $\alpha=-18^{\circ}$

$C_{p}$ VERSUS $x / c$

NASA LS(1) -0417 MOD $R e=1 . \times 10^{\circ}$

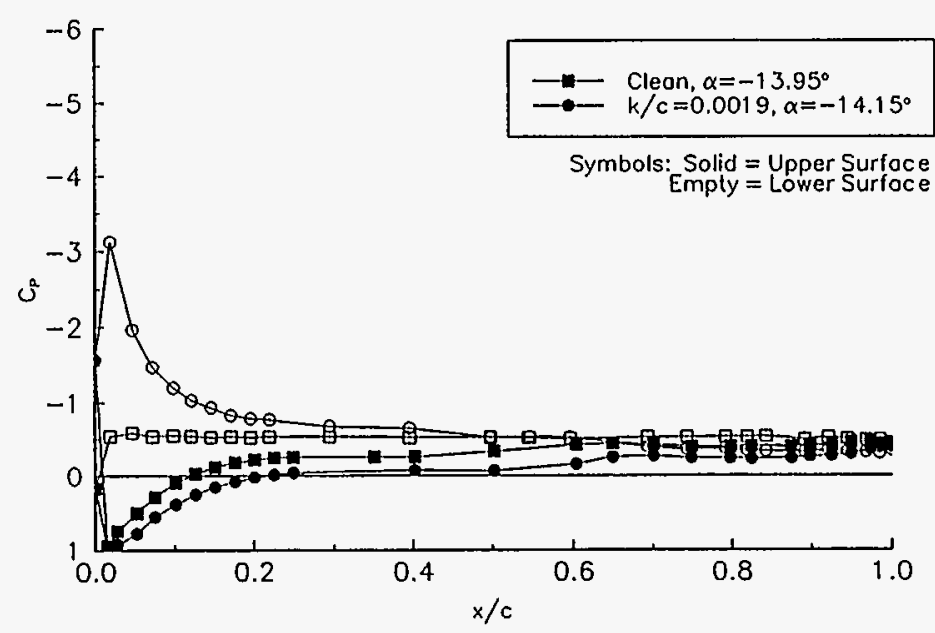

Figure 15. $\alpha=-14^{\circ}$
$C_{\mathrm{p}}$ VERSUS $x / \mathrm{c}$

NASA LS(1)-0417 MOD $R e=1 . \times 10^{\circ}$

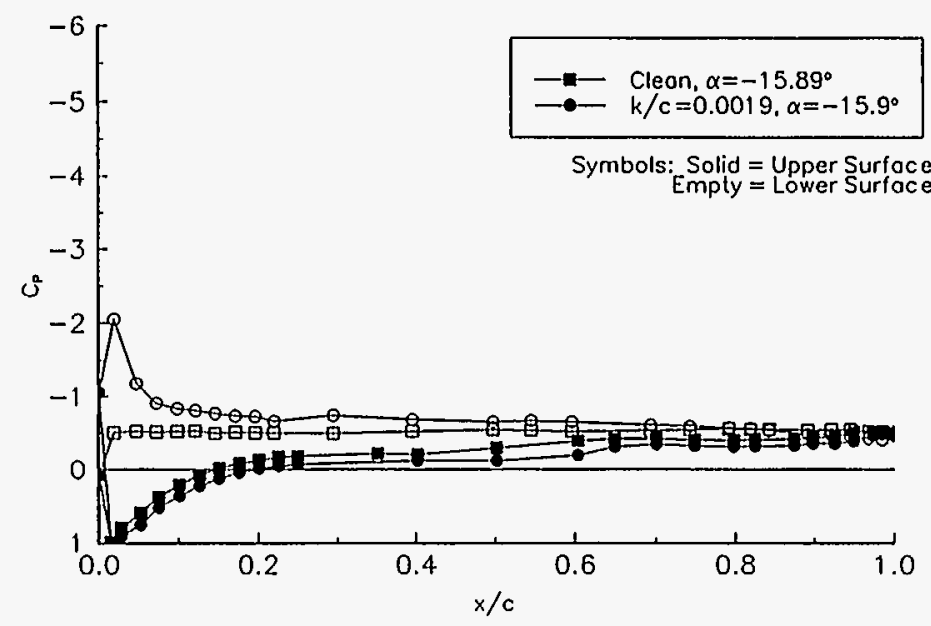

Figure 14. $\alpha=-16^{\circ}$

$C_{P}$ VERSUS $x / c$

NASA LS(1) -0417 MOD $R e=1 . \times 10^{\circ}$

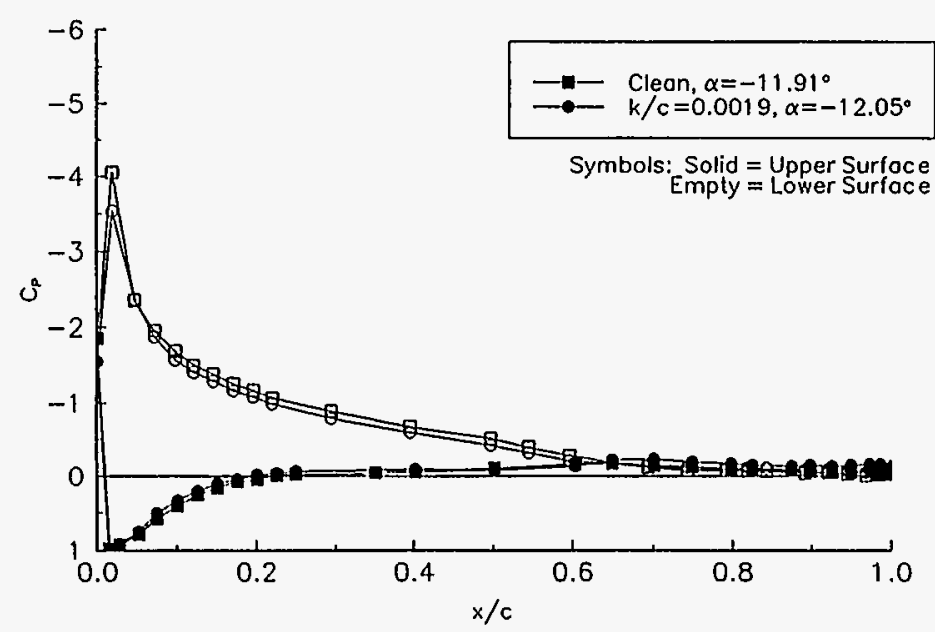

Figure 16. $\alpha=-12^{\circ}$ 
$C_{p}$ VERSUS $x / c$

NASA LS(1) -0417 MOD $\operatorname{Re}=1 . \times 10^{\circ}$

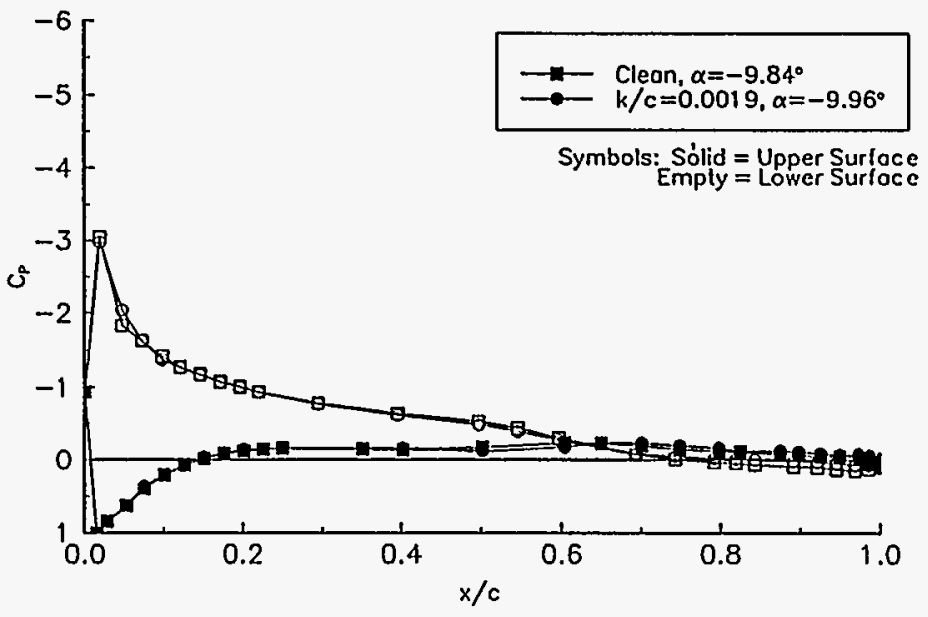

Figure 17. $\alpha=-10^{\circ}$

$G_{p}$ VERSUS $\times / c$

NASA LS(1) -0417 MOD $R e=1 . \times 10^{6}$

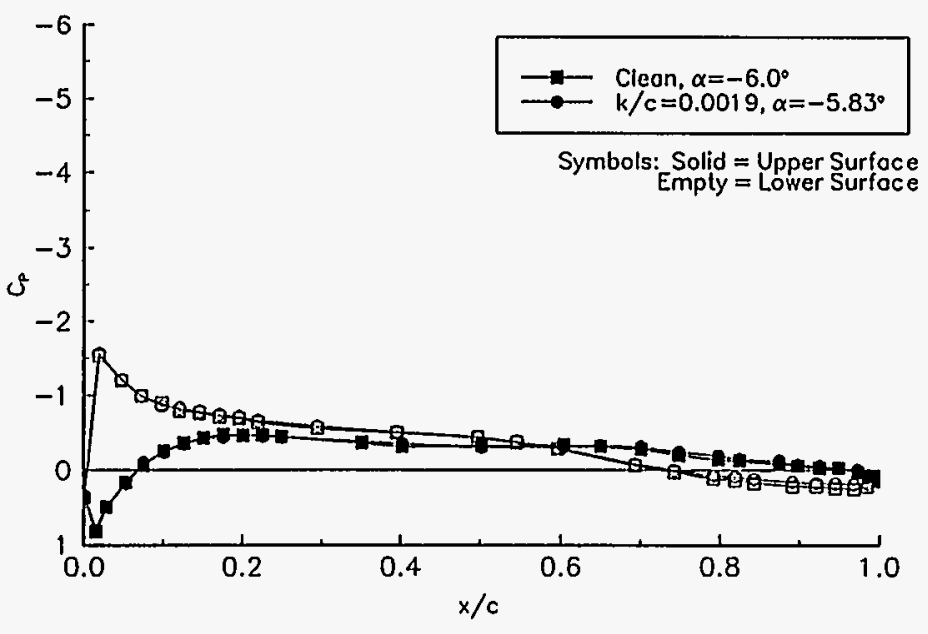

Figure 19. $\alpha=-6^{\circ}$
$C_{p}$ VERSUS $x / c$

NASA LS(1) -0417 MOD Re $=1 . \times 10^{6}$

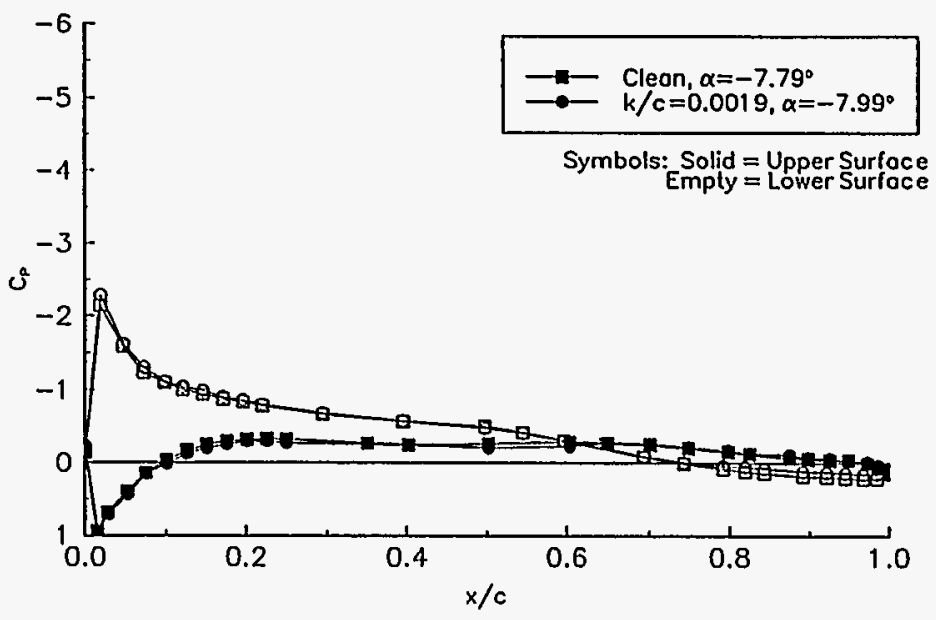

Figure 18. $\alpha=-8^{\circ}$

$C_{p}$ VERSUS $x / c$ NASA LS(1) $-0417 \mathrm{MOD}$ Re $=1 . \times 10^{8}$

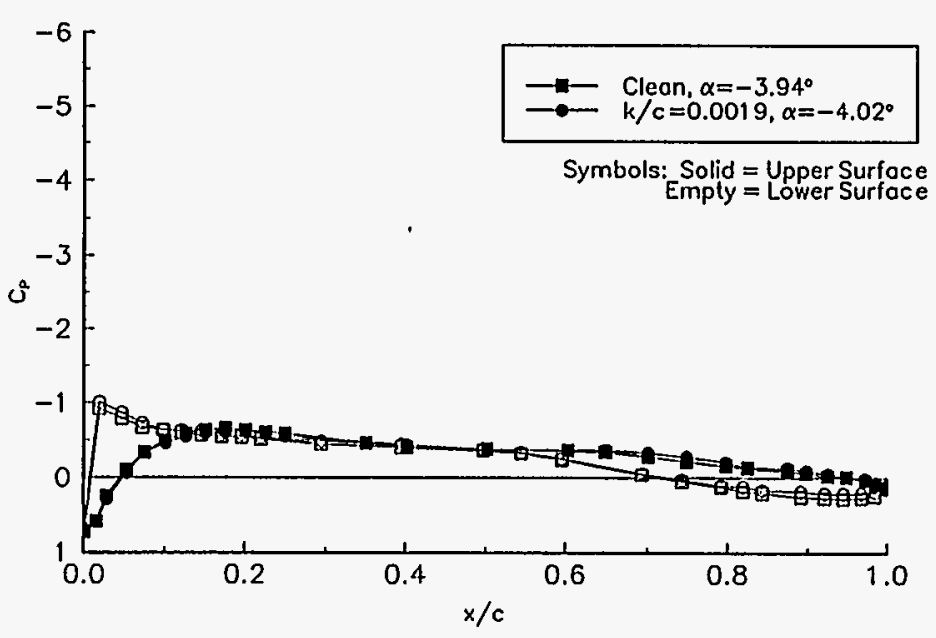

Figure 20. $\alpha=-4^{\circ}$ 
$C_{p}$ VERSUS $x / c$

NASA LS(1)-0417 MOD $\operatorname{Re}=1 . \times 10^{6}$

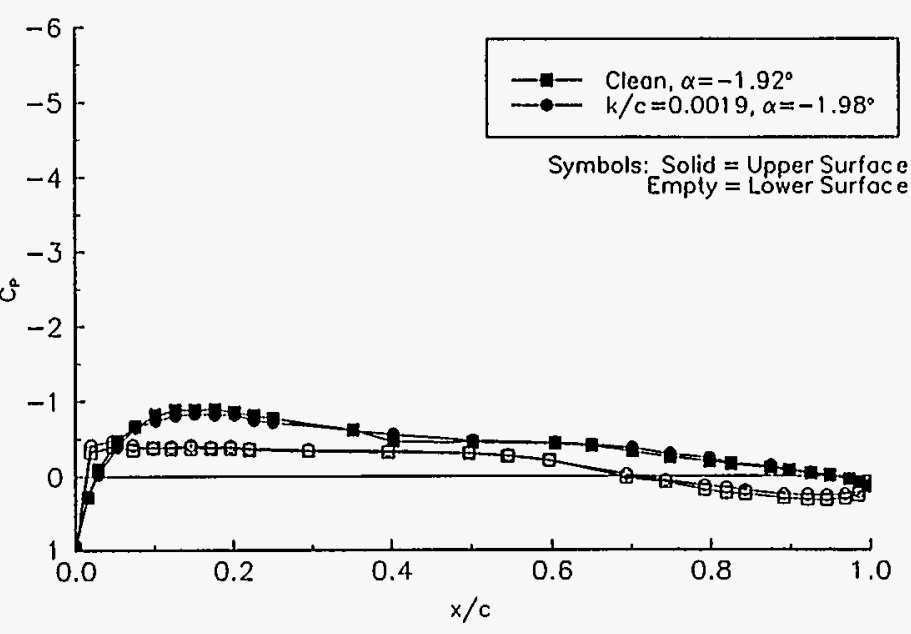

Figure 21. $\alpha=-2^{\circ}$

$C_{p}$ VERSUS $x / c$

$\operatorname{NASALS}(1)-0417$ MOO Re $=1 . \times 10^{6}$

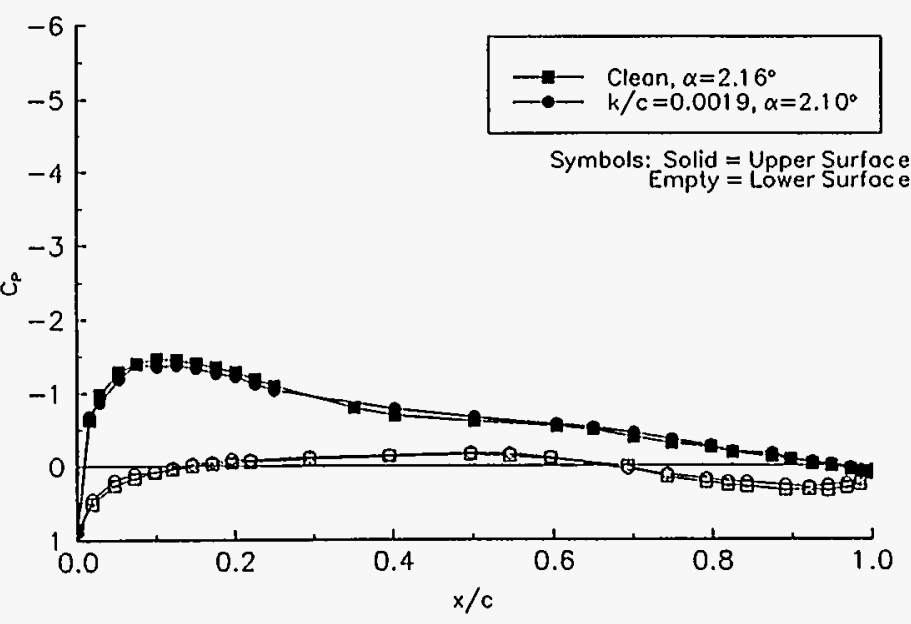

Figure 23. $\alpha=2^{\circ}$
$C_{p}$ VERSUS $x / C$

NASA LS(1)-0417 MOD Re $=1 . \times 10^{6}$

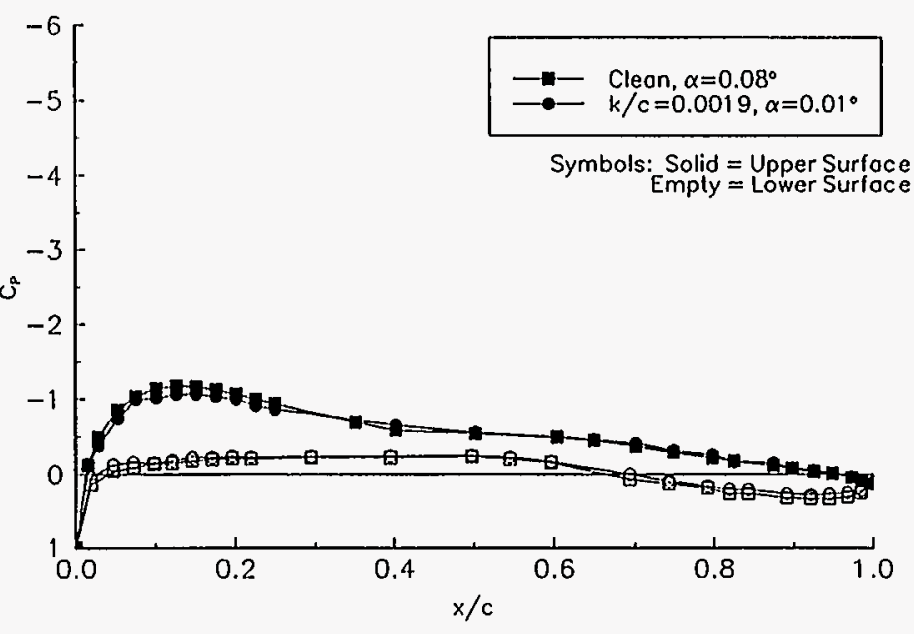

Figure 22. $\alpha=0^{\circ}$

$C_{p}$ VERSUS $x / c$

NASA LS(1) -0417 MOD $R e=1 . \times 10^{\circ}$

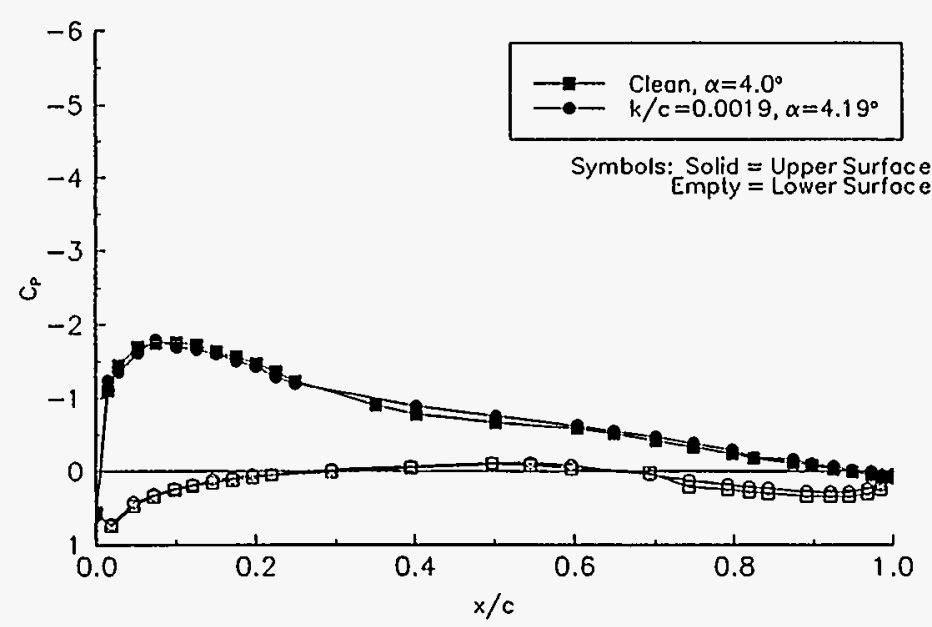

Figure 24. $\alpha=4^{\circ}$ 

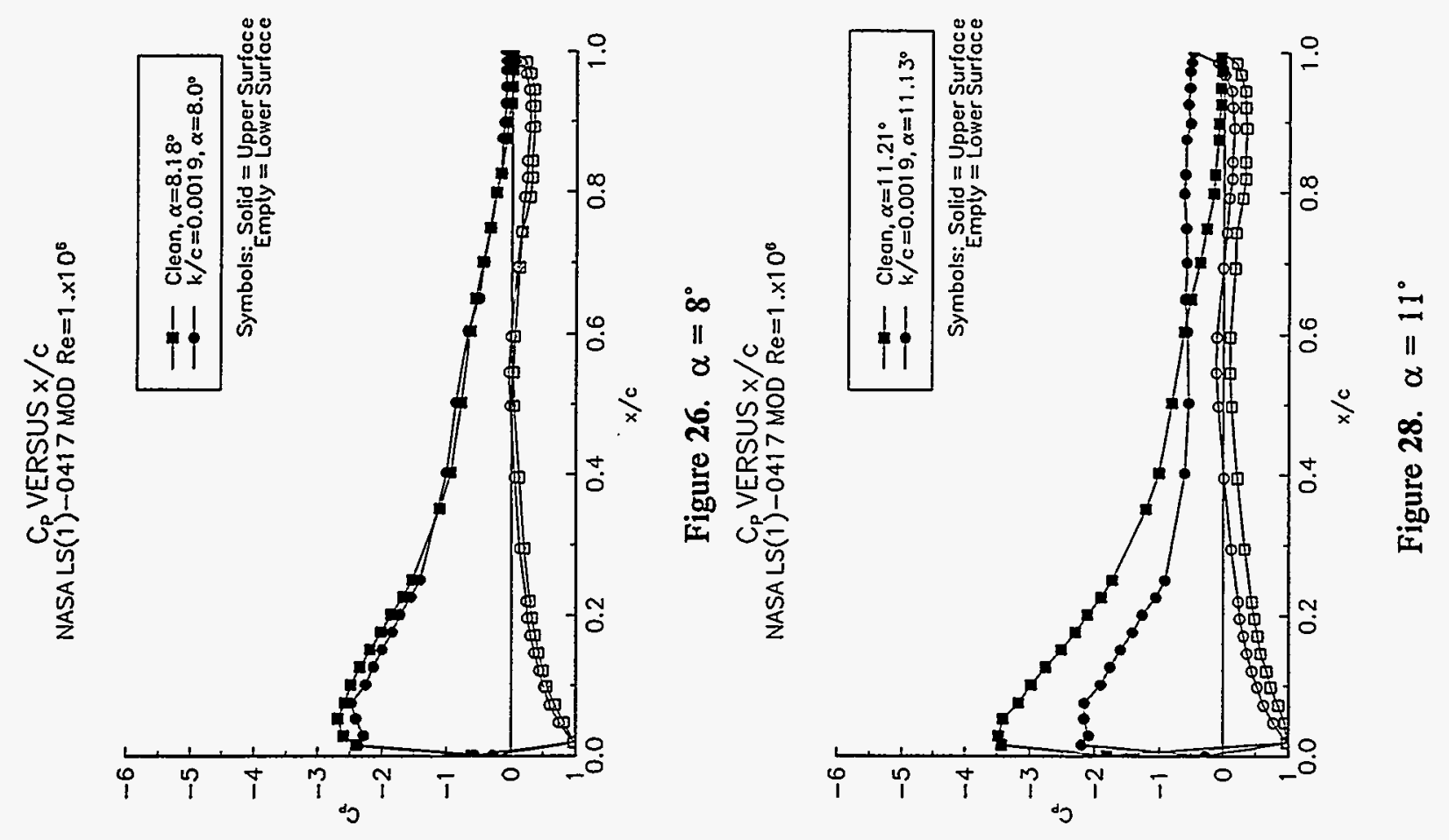

早
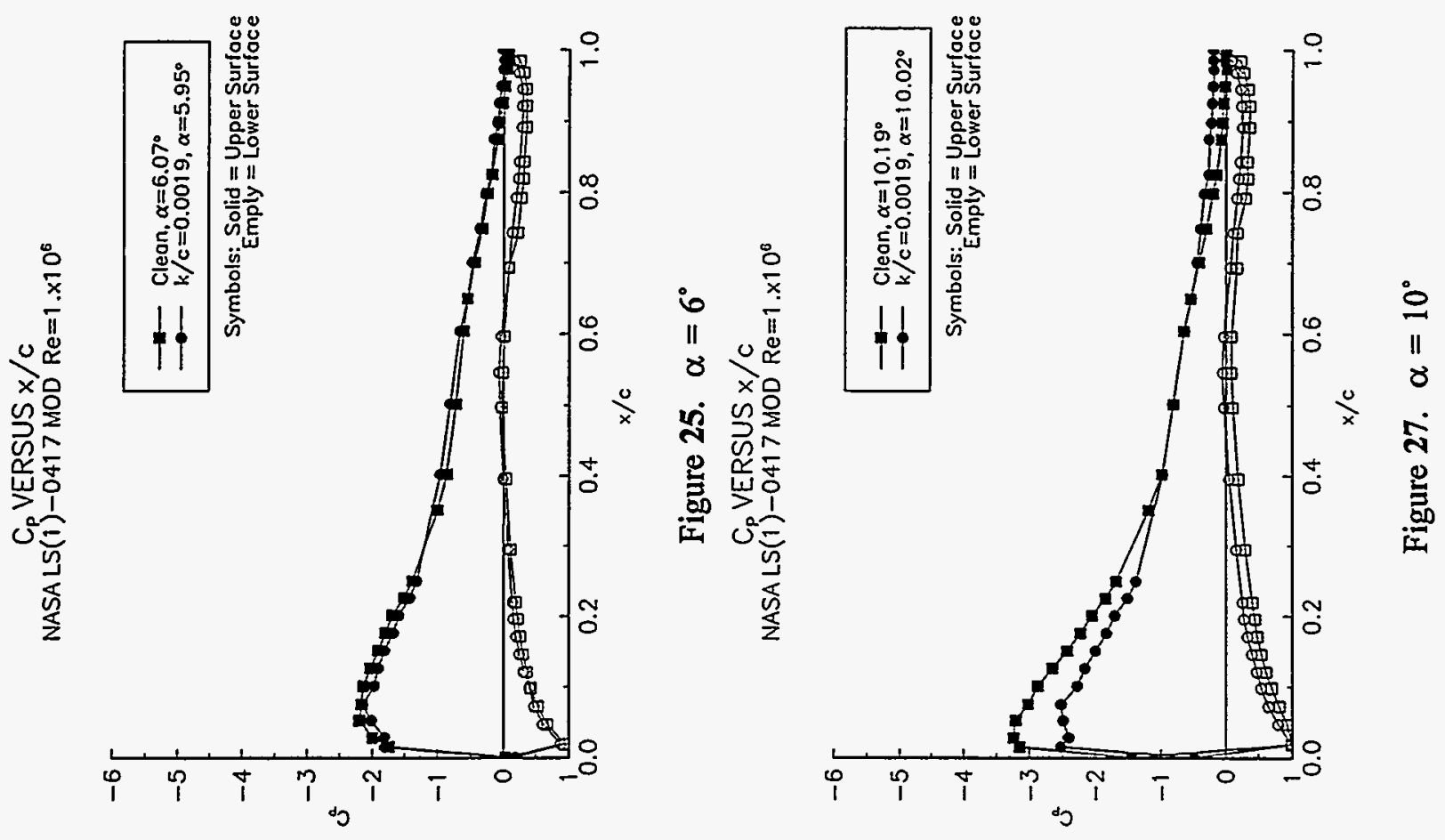
$C_{p}$ VERSUS $x / C$

NASALS(1)-0417 MOD Re $=1 . \times 10^{\circ}$

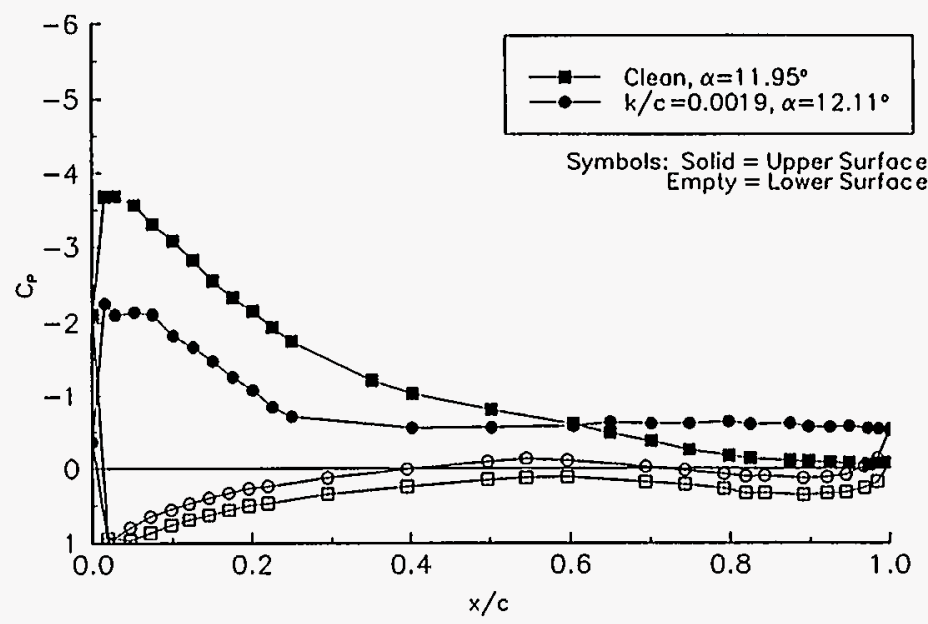

Figure 29. $\alpha=12^{\circ}$

$C_{p}$ VERSUS $x / c$

$\operatorname{NASALS}(1)-0417$ MOD $\operatorname{Re}=1 . \times 10^{\circ}$

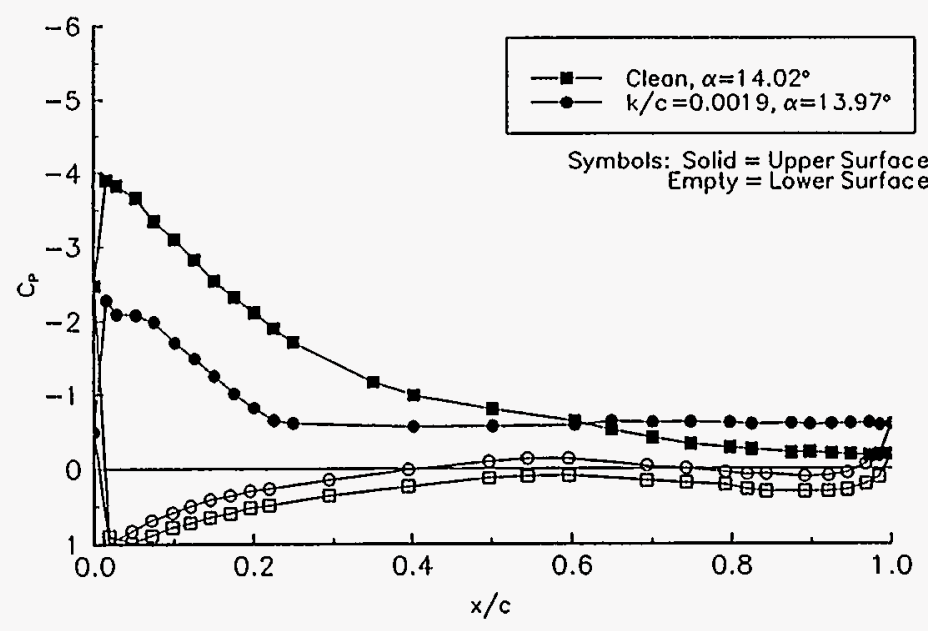

Figure 31. $\alpha=14^{\circ}$
$C_{p}$ VERSUS $x / c$

NASA LS(1)-0417 MOD Re $=1 . \times 10^{6}$

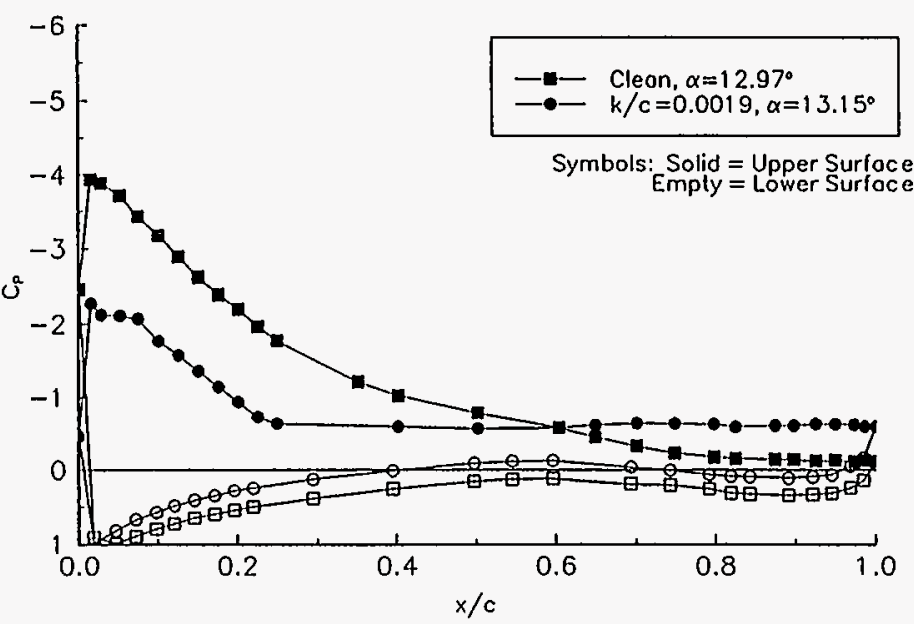

Figure 30. $\alpha=13^{\circ}$

$C_{P}$ VERSUS $x / c$

NASA LS(1)-0417 MOD Re $=1 . \times 10^{\circ}$

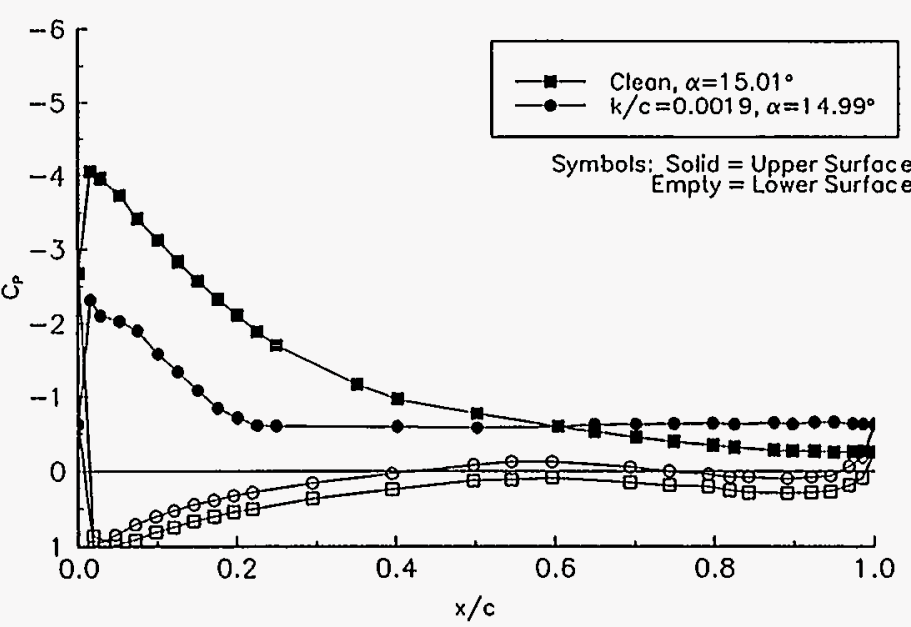

Figure 32. $\alpha=15^{\circ}$ 

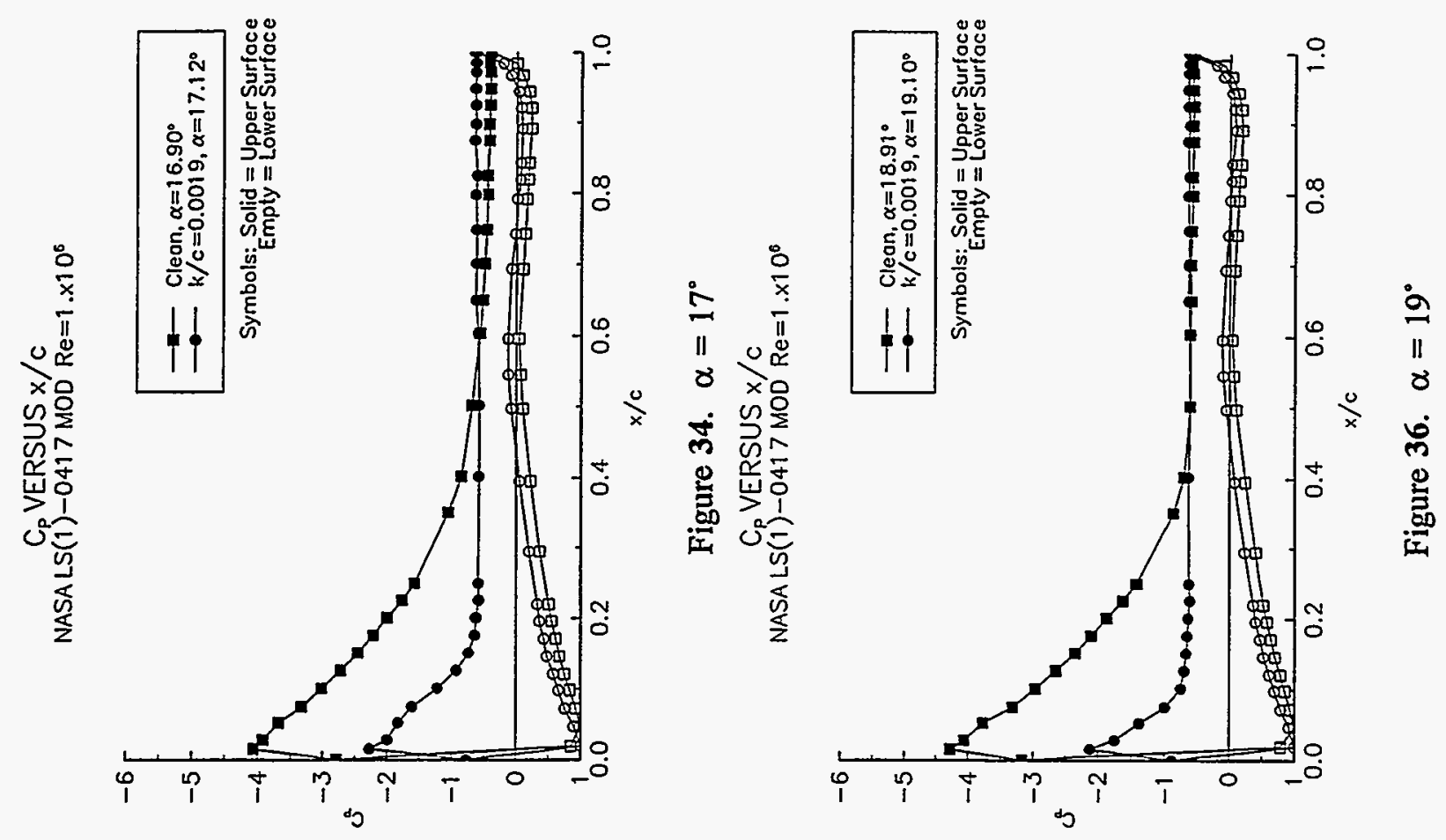

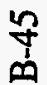
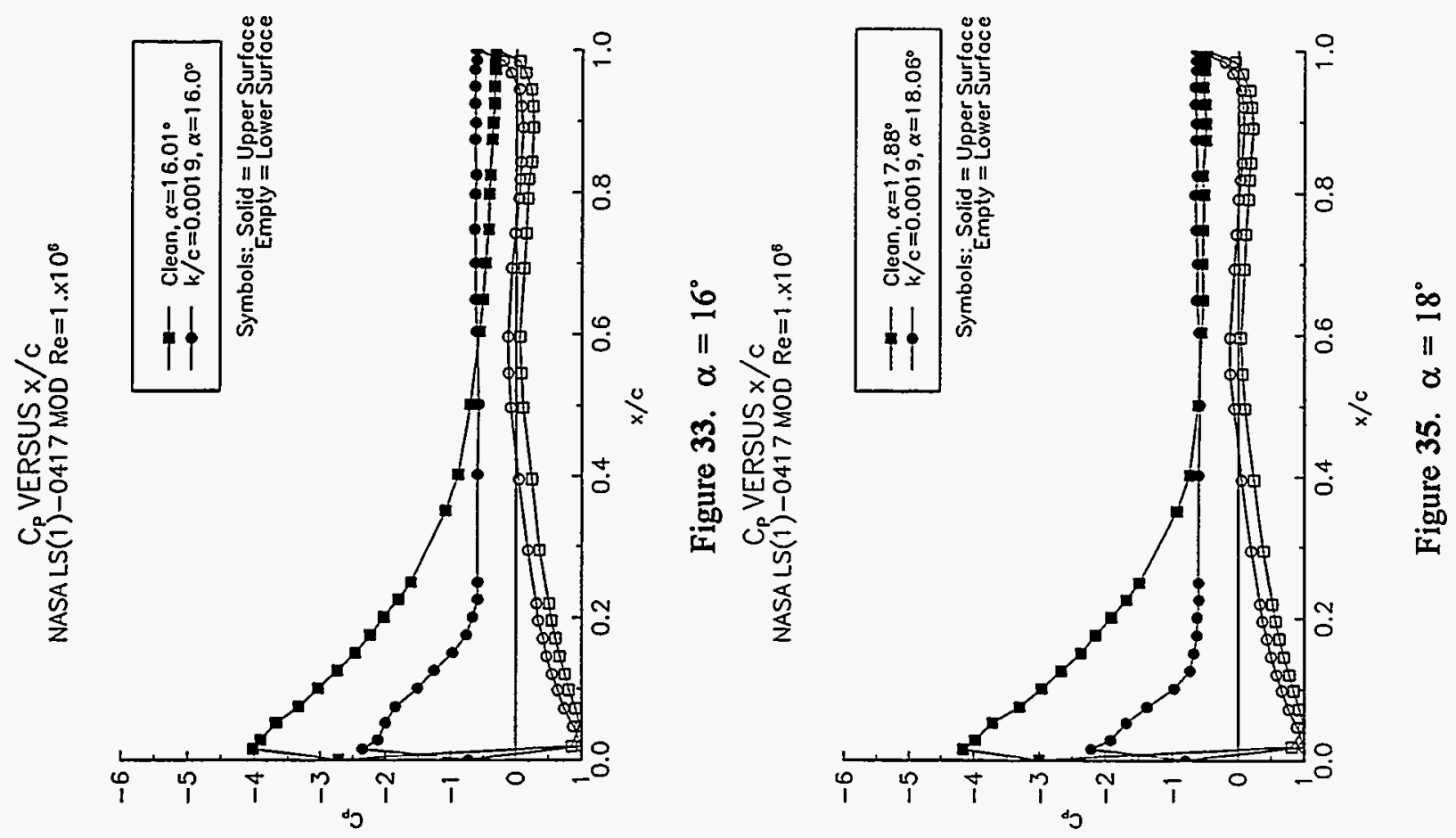
$C_{p}$ VERSUS $x / c$

NASA LS(1) -0417 MOD $R e=1 . \times 10^{\circ}$

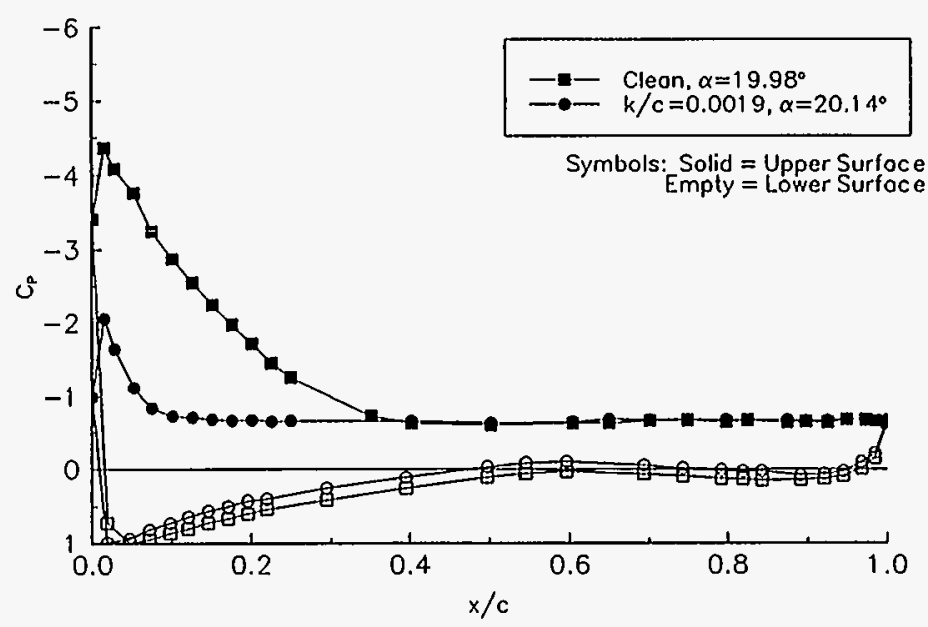

Figure 37. $\alpha=20^{\circ}$

$C_{p}$ VERSUS $x / c$

NASA LS(1)-0417 MOD $\operatorname{Re}=1 . \times 10^{6}$

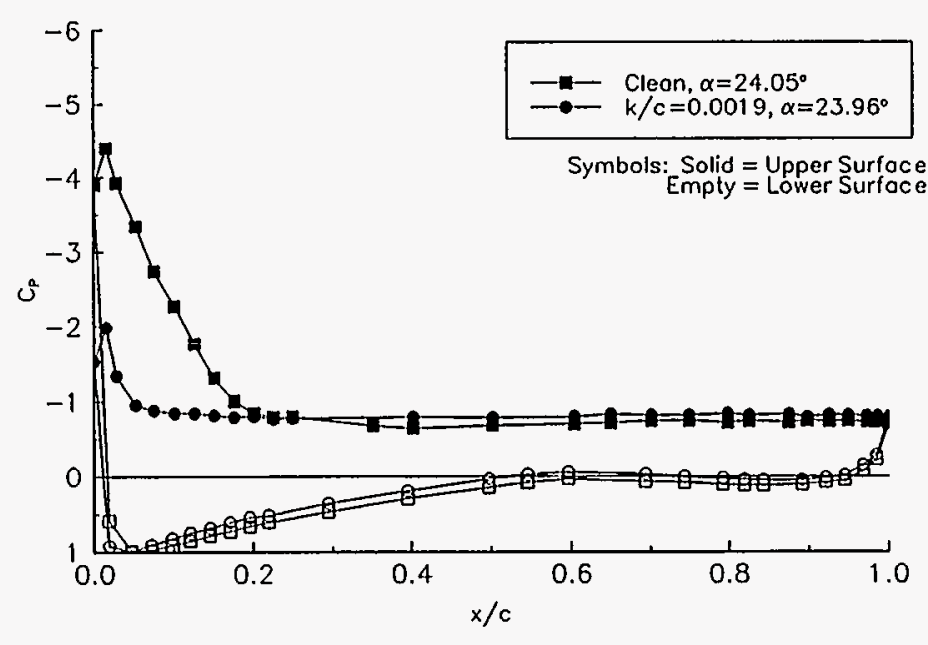

Figure 39. $\alpha=24^{\circ}$
$C_{p}$ VERSUS $x / c$

NASALS(1)-0417MOO Re $=1 . \times 10^{6}$

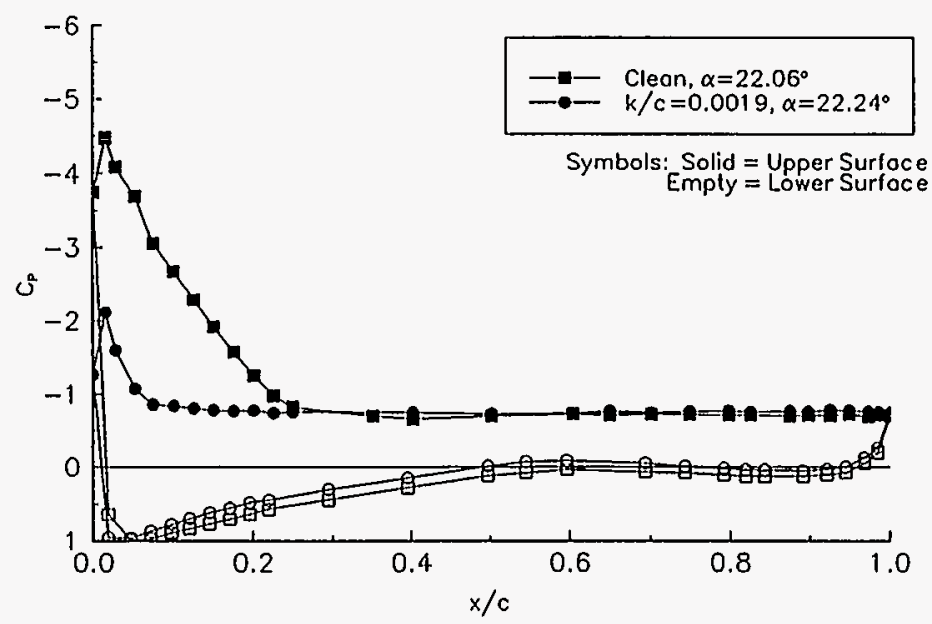

Figure 38. $\alpha=22^{\circ}$

$C_{p}$ VERSUS $x / c$

NASA LS(1)-0417 MOD Re $=1 . \times 10^{6}$

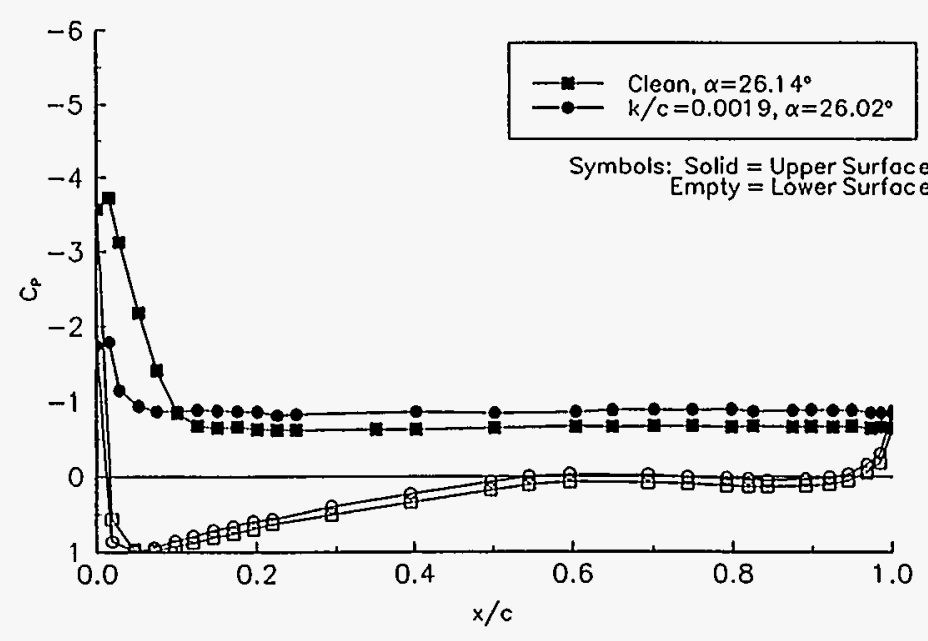

Figure 40. $\alpha=26^{\circ}$ 
$C_{p}$ VERSUS $x / C$

NASA LS(1) -0417 MOD $R e=1 . \times 10^{8}$

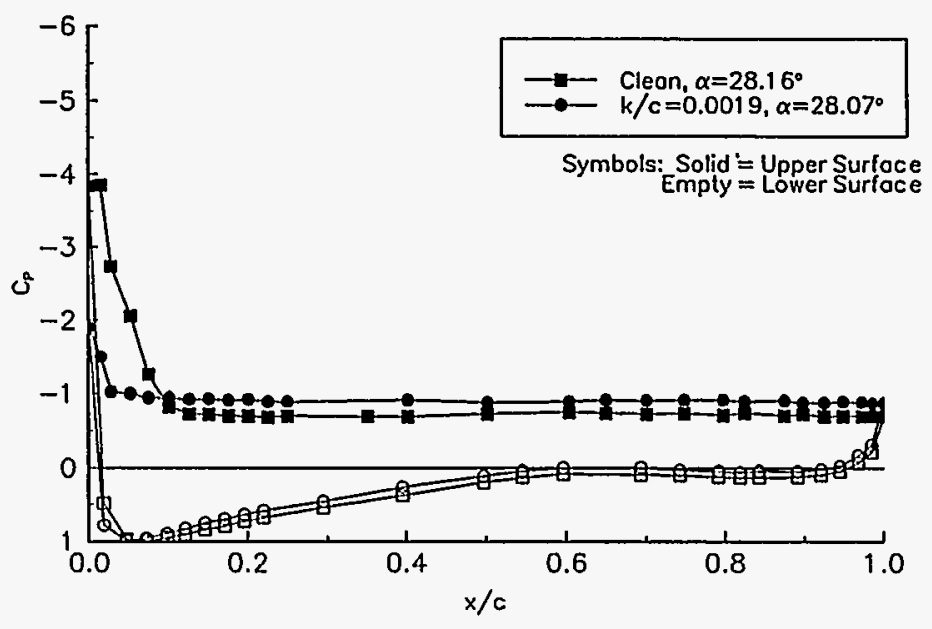

Figure 41. $\alpha=28^{\circ}$

$C_{p}$ VERSUS $x / c$

NASA LS(1) -0417 MOD $R e=1 . \times 10^{6}$

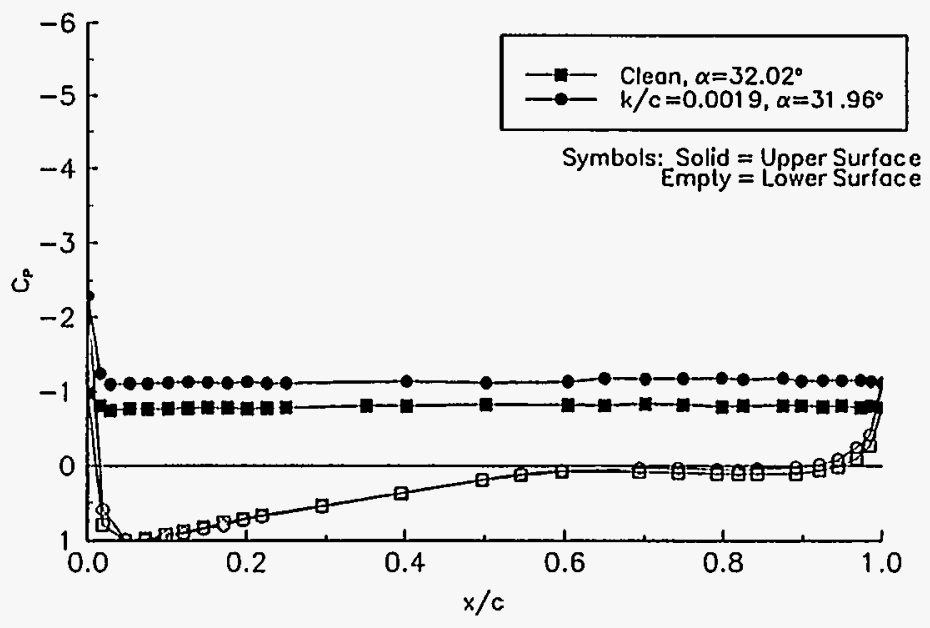

Figure 43. $\alpha=32^{\circ}$
$C_{p}$ VERSUS $x / c$

NASALS(1) -0417 MOD $R e=1 . \times 10^{6}$

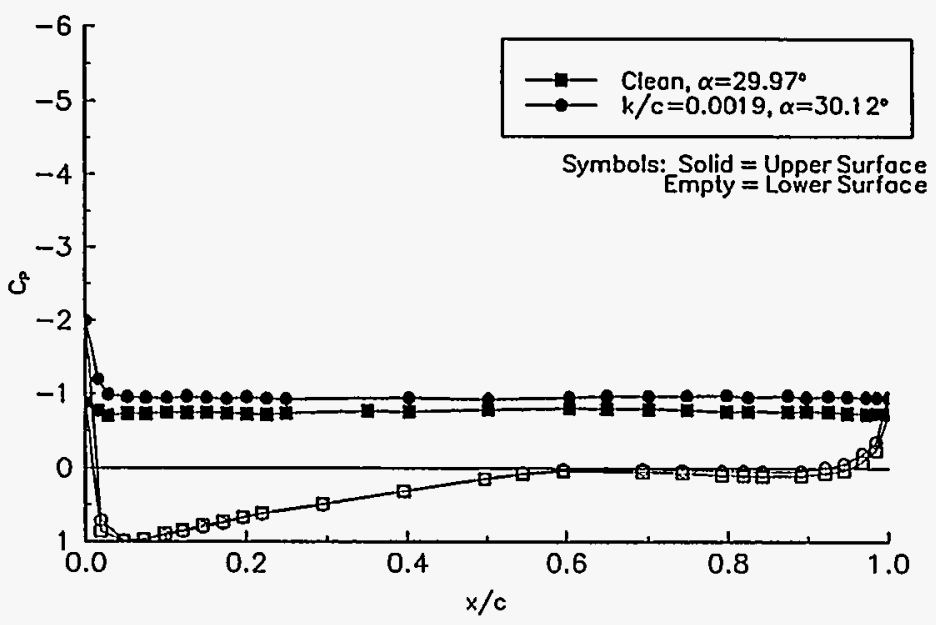

- Figure 42. $\alpha=30^{\circ}$

$C_{p}$ VERSUS $x / c$ NASA LS(1) -0417 MOD $R e=1 . \times 10^{6}$

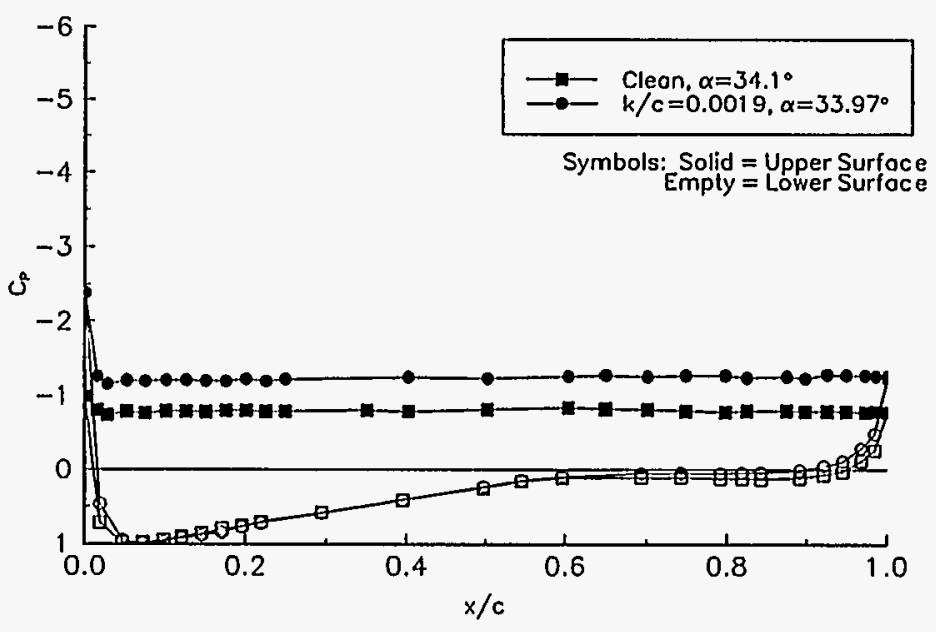

Figure 44. $\alpha=34^{\circ}$ 
$C_{p}$ VERSUS $x / c$

NASA LS (1) -0417 MOO $\operatorname{Re}=1 . \times 10^{\circ}$

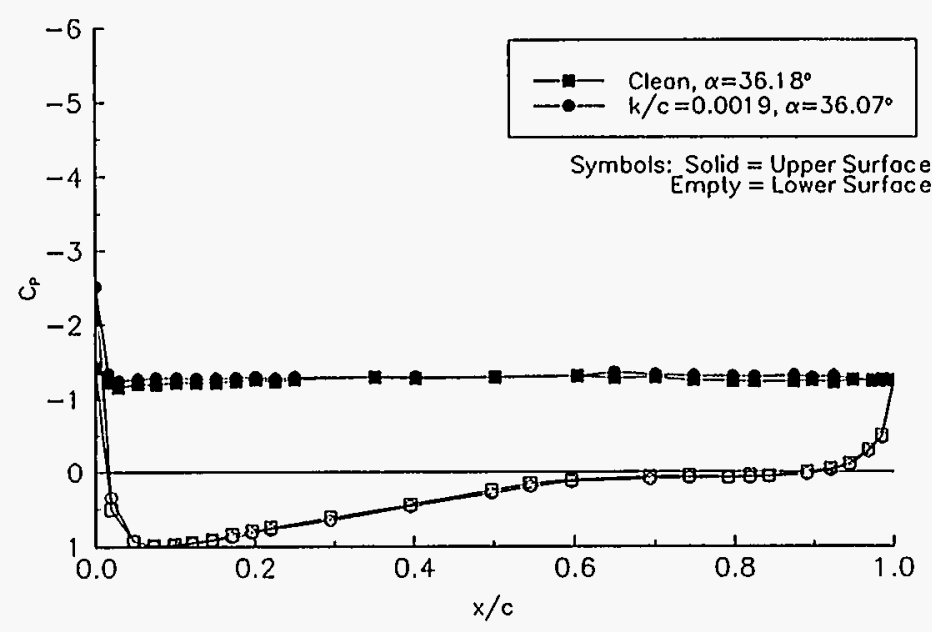

Figure 45. $\alpha=36^{\circ}$

$C_{p}$ VERSUS $x / c$

NASA LS (1)-0417 MOD Re $=1 . \times 10^{\circ}$

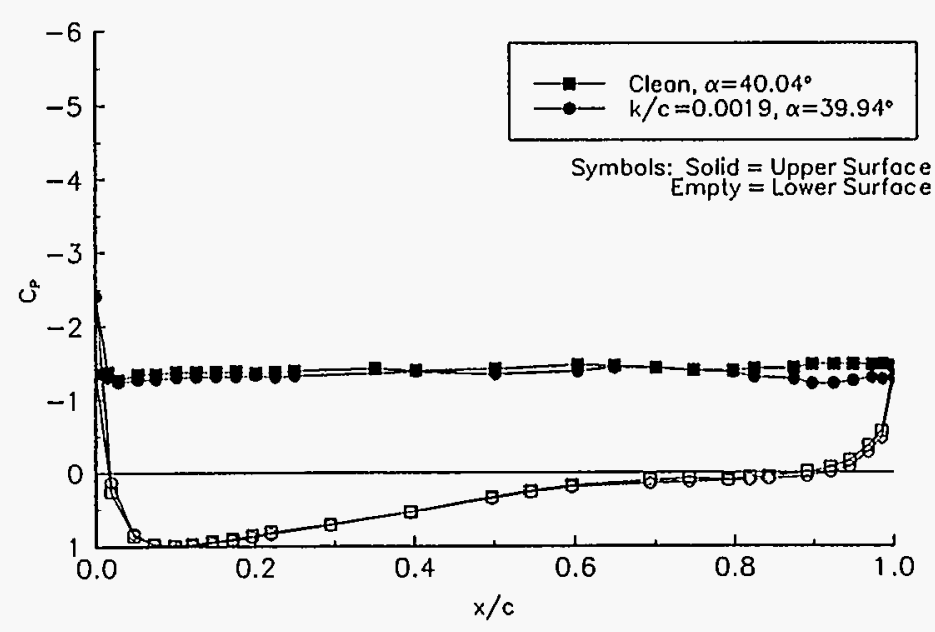

Figure 47. $\alpha=40^{\circ}$
$C_{p}$ VERSUS $x / c$

NASA LS(1)-0417 MOD $R e=1 . \times 10^{6}$

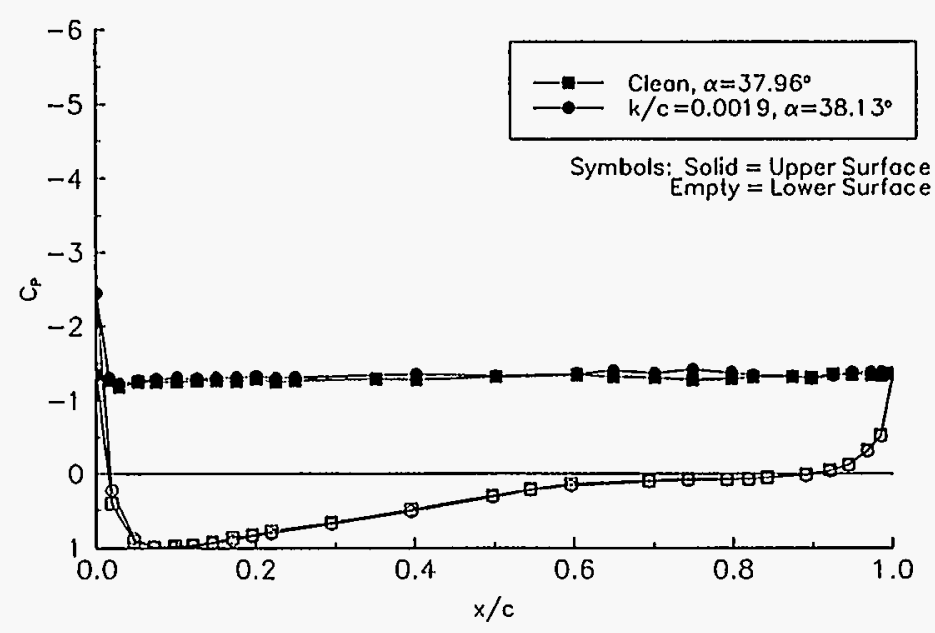

Figure 46. $\alpha=38^{\circ}$

$C_{p}$ VERSUS $x / c$

NASA LS (1) -0417 MOD $R e=1 . \times 10^{\circ}$

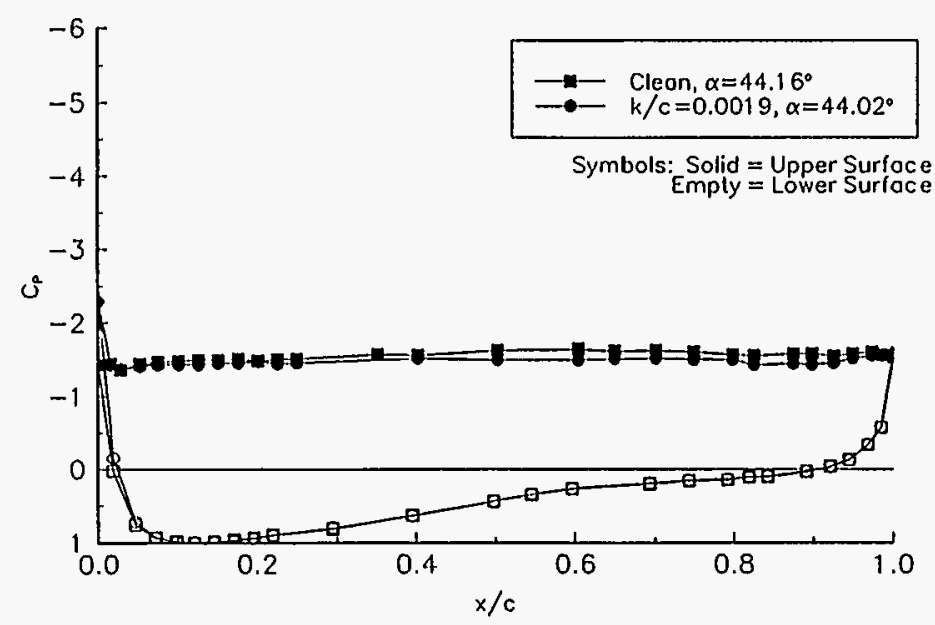

Figure 48. $\alpha=44^{\circ}$ 


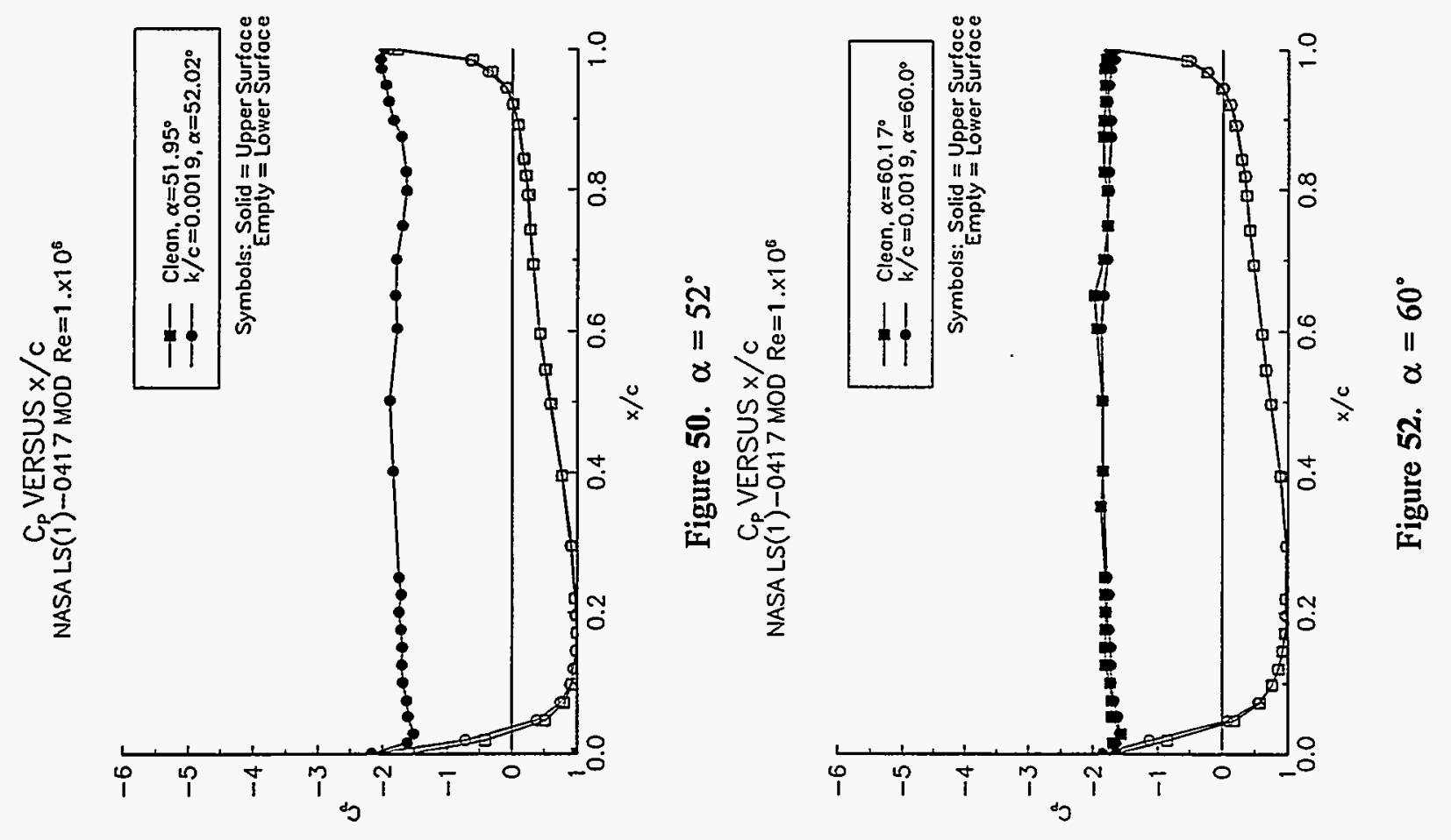

䯩

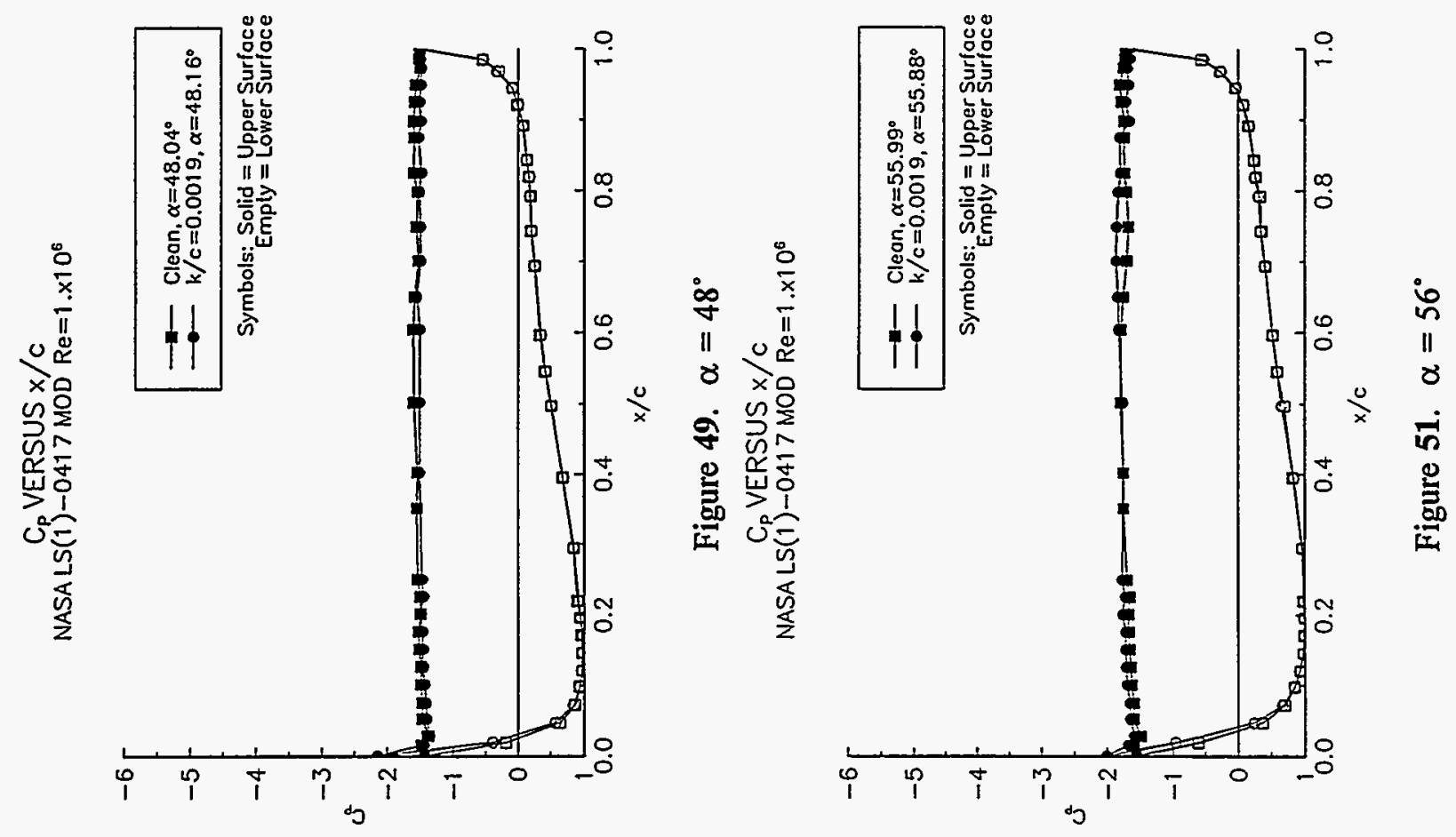


$C_{p}$ VERSUS $x / c$

NASA LS(1)-0417 MOD Re $=1 . \times 10^{6}$

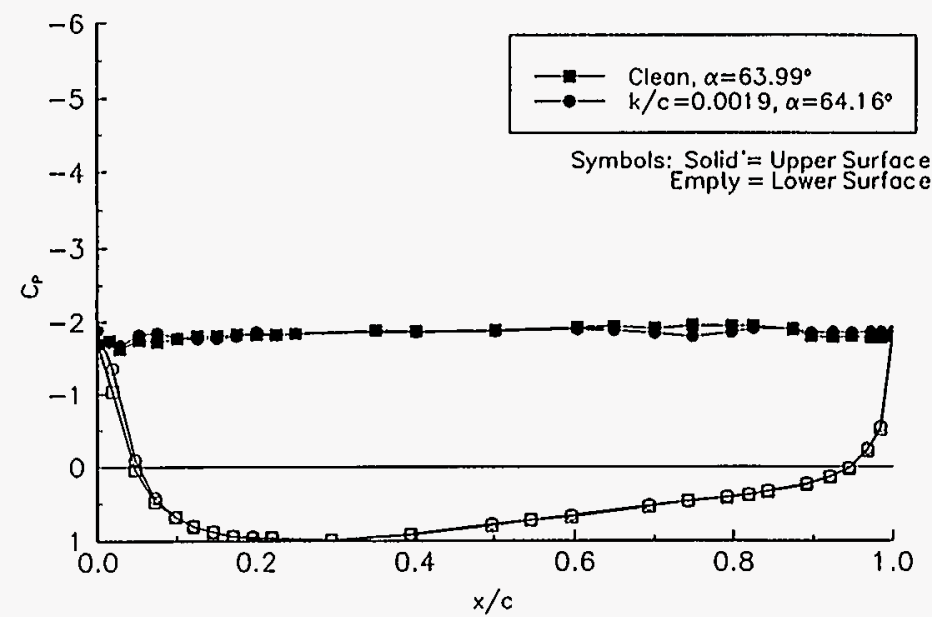

Figure 53. $\alpha=64^{\circ}$

$C_{p}$ VERSUS $x / c$

NASALS(1) -0417 MOD $R e=1 . \times 10^{\circ}$

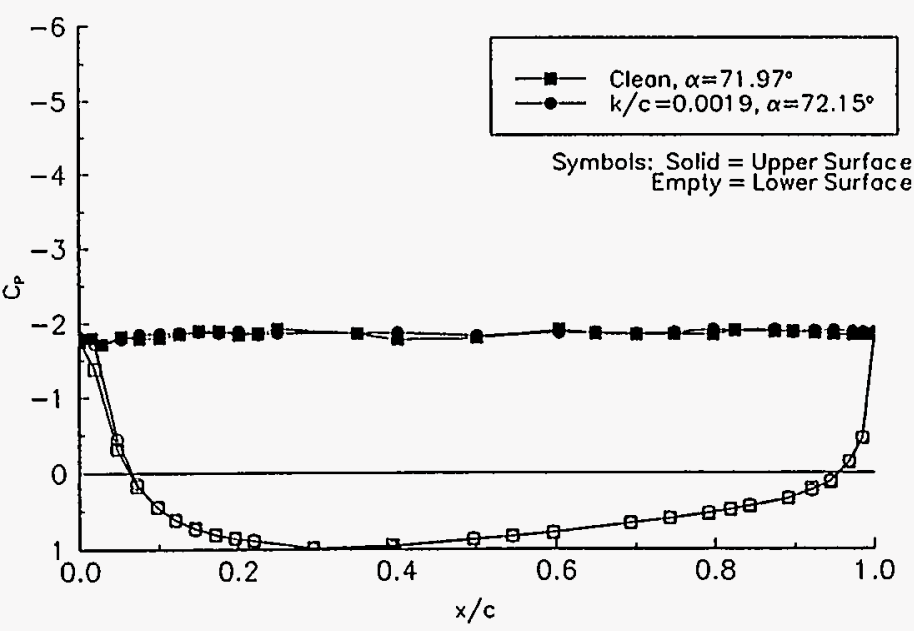

Figure 55. $\alpha=72^{\circ}$
$C_{p}$ VERSUS $x / c$

NASA LS(1) -0417 MOD $R e=1 . \times 10^{\circ}$

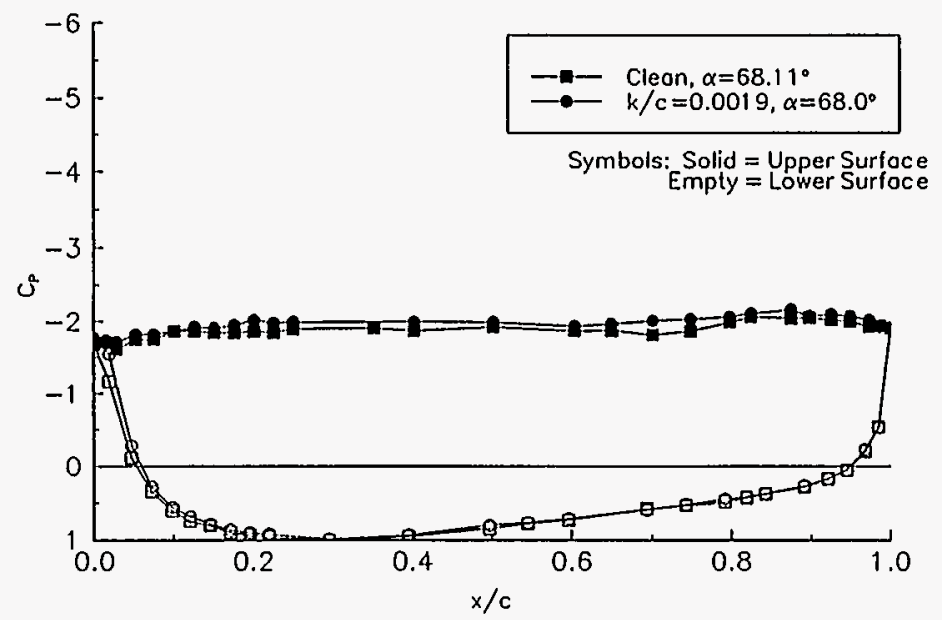

Figure 54. $\alpha=68^{\circ}$

$C_{p}$ VERSUS $x / c$

NASA LS(1) -0417 MOD $R e=1 . \times 10^{\circ}$

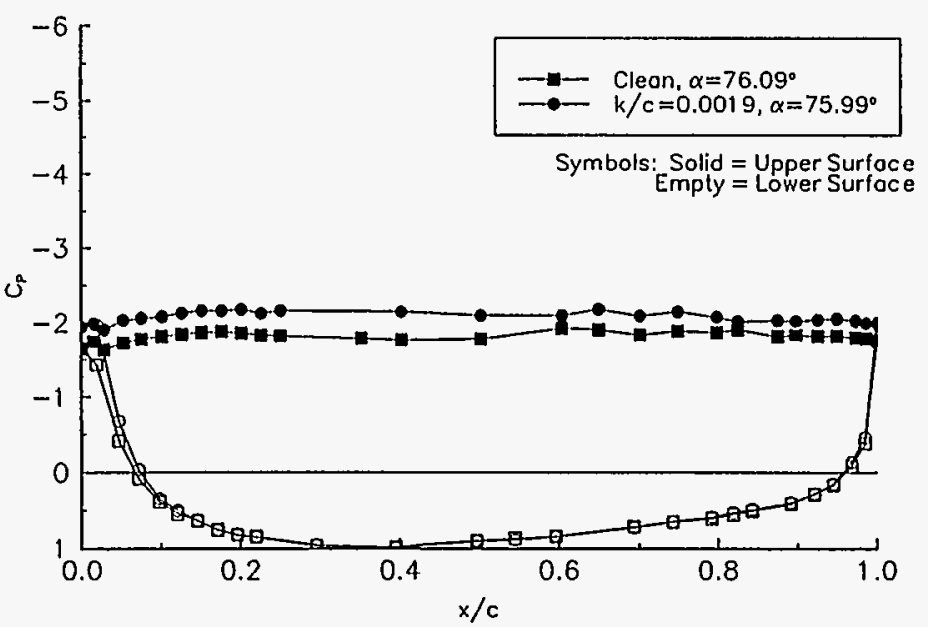

Figure 56. $\alpha=76^{\circ}$ 

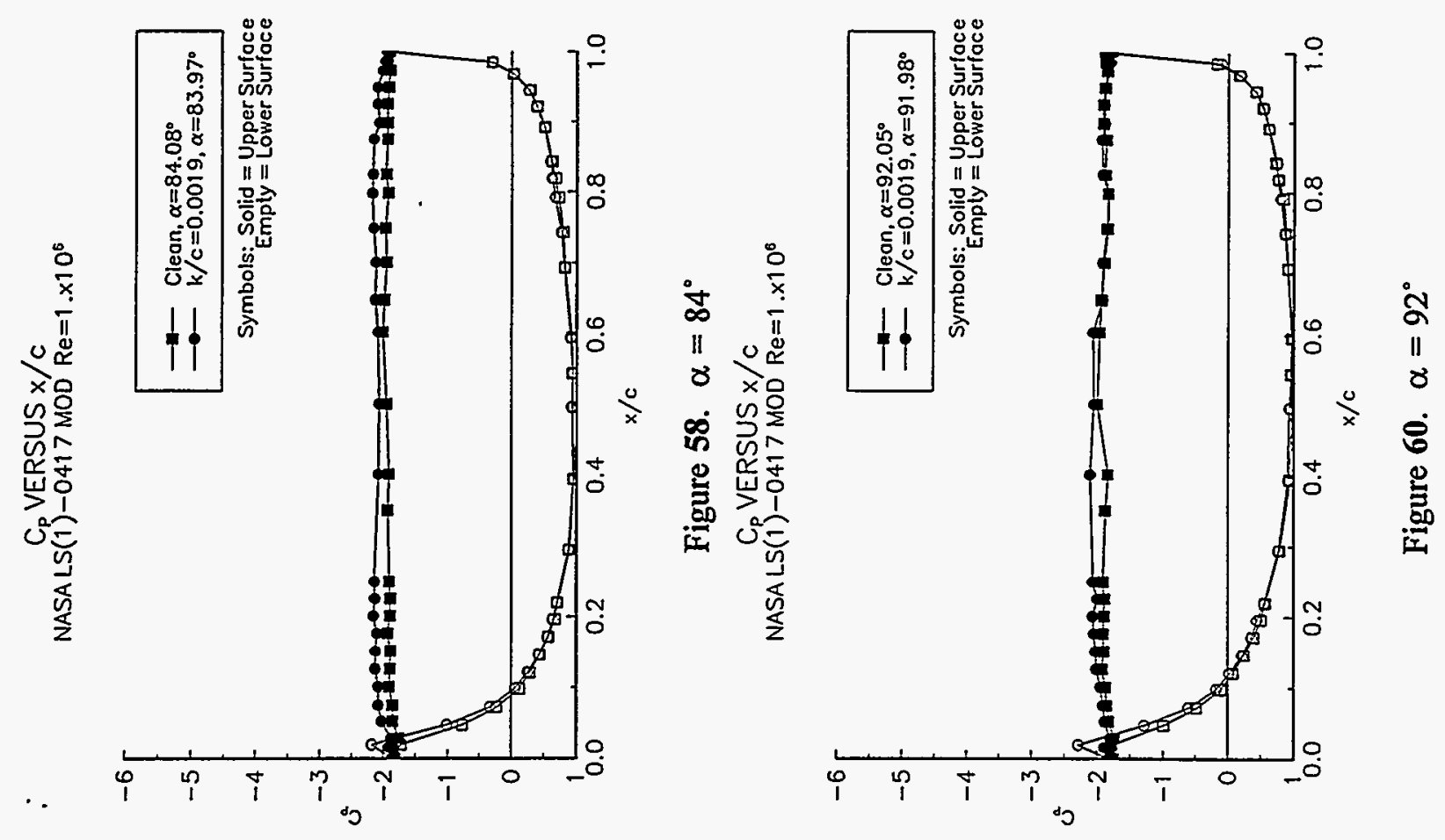

$\vec{\Phi}$
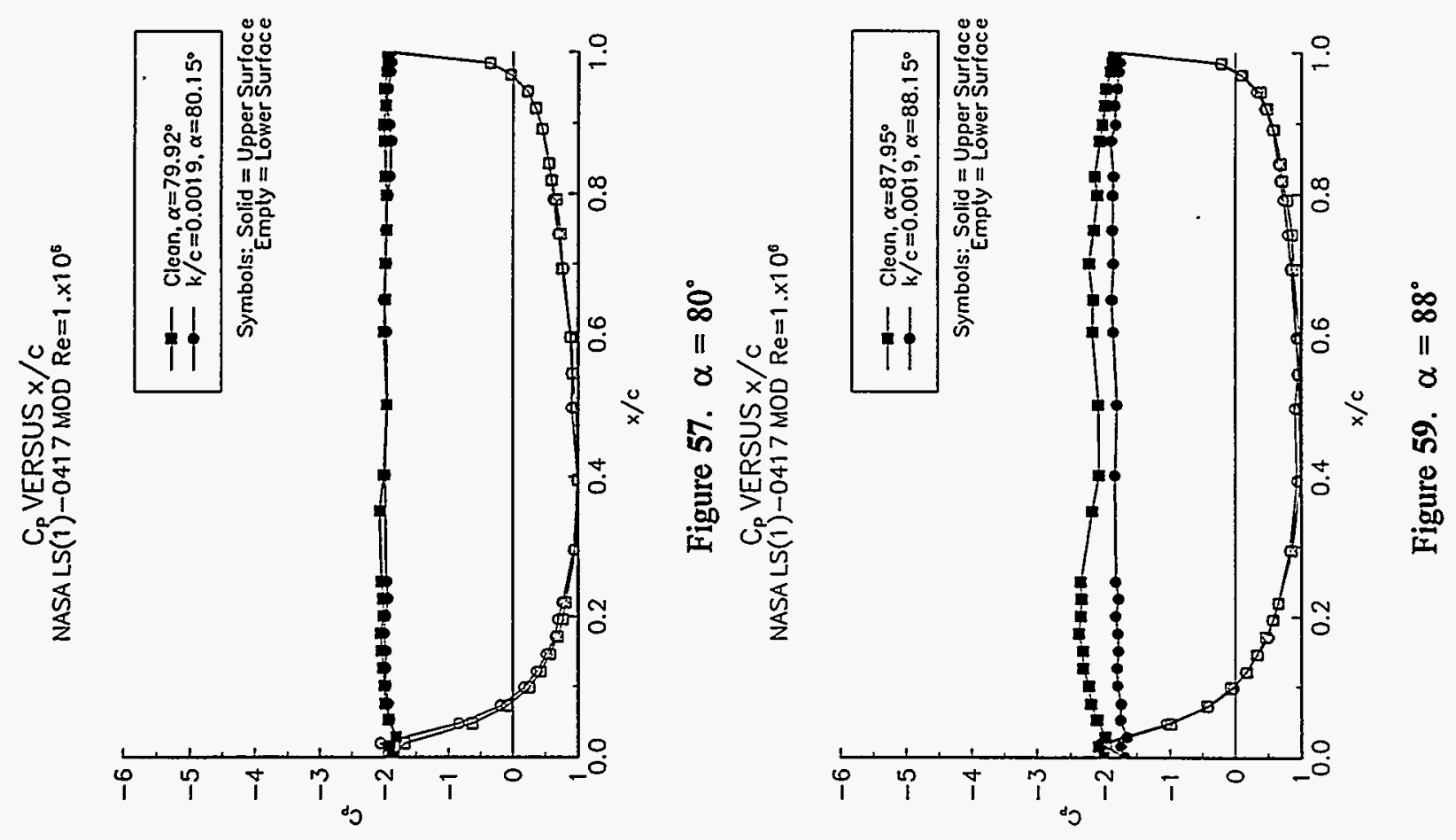
$C_{p}$ VERSUS $x / c$

NASA LS(1)-0417 MOD $R e=1 . \times 10^{6}$

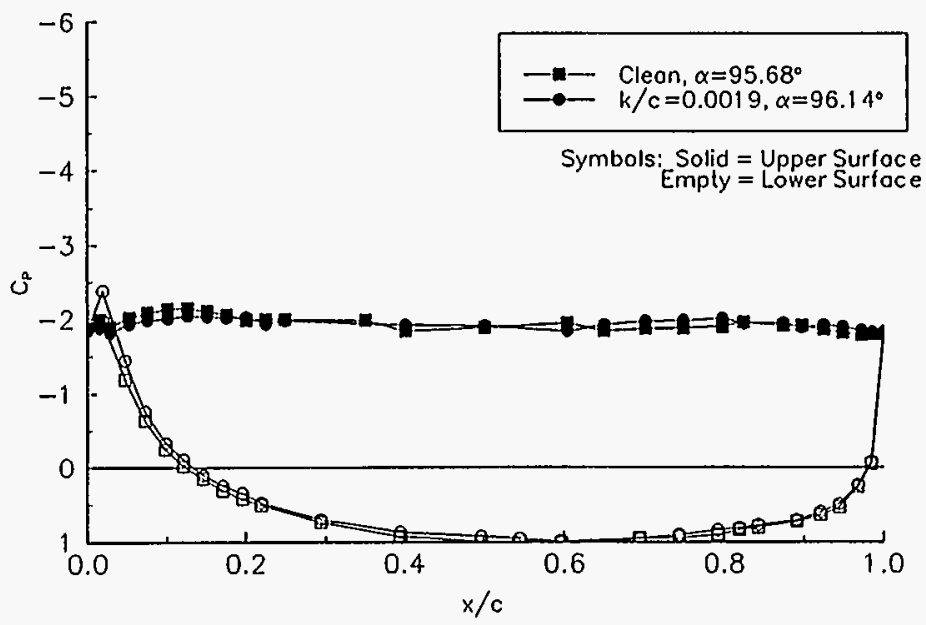

Figure 61. $\alpha=96^{\circ}$

$C_{p}$ VERSUS $x / c$

NASA LS(1)-0417 MOD $\operatorname{Re}=1 . \times 10^{6}$

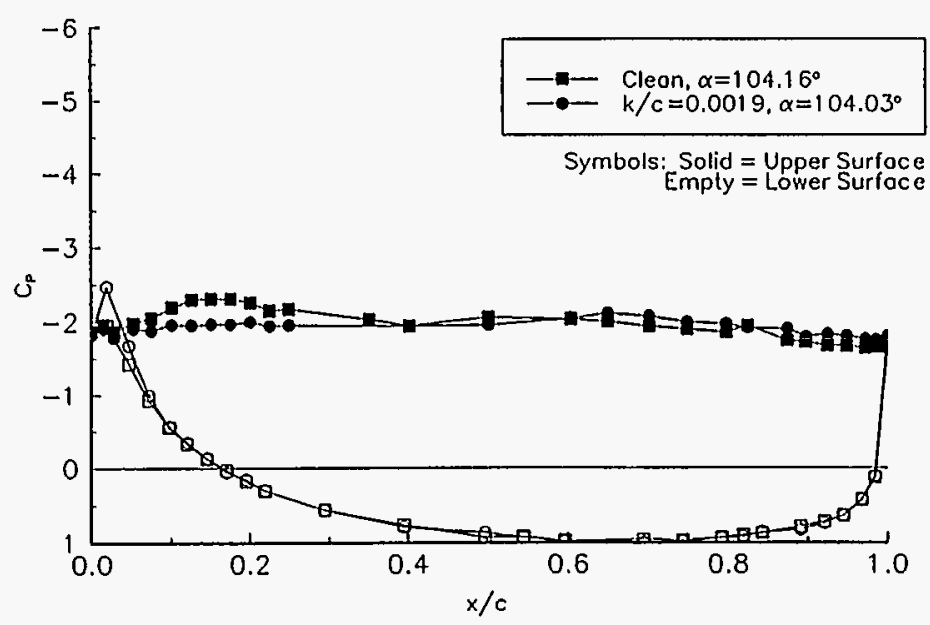

Figure 63. $\alpha=104^{\circ}$
$C_{p}$ VERSUS $x / c$

NASA LS(1)-0417 MOD $R e=1 . \times 10^{\circ}$

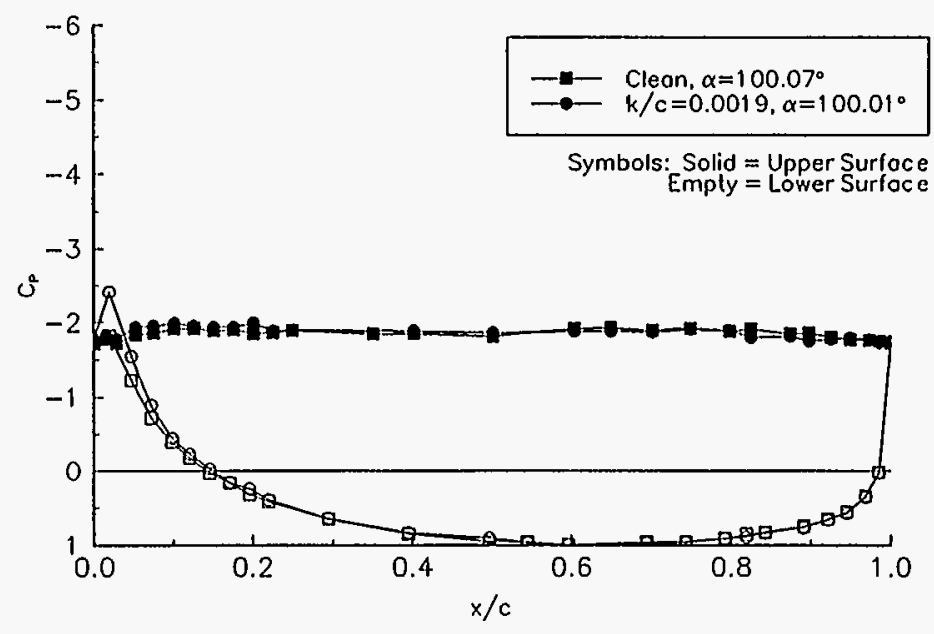

Figure 62. $\alpha=100^{\circ}$

$C_{p}$ VERSUS $\times / c$

NASA LS(1)-0417 MOD Re $=1 . \times 10^{\circ}$

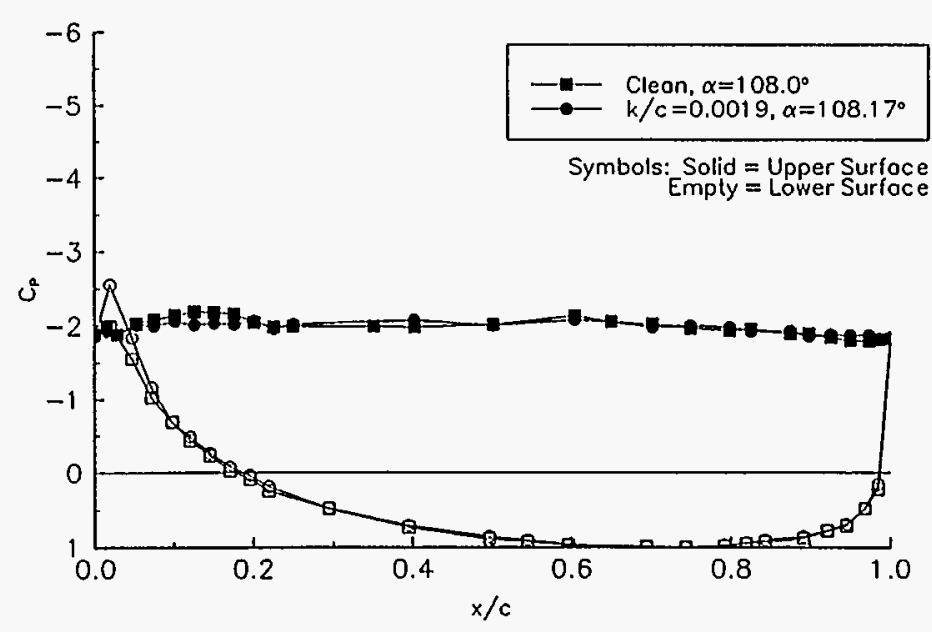

Figure 64. $\alpha=108^{\circ}$ 


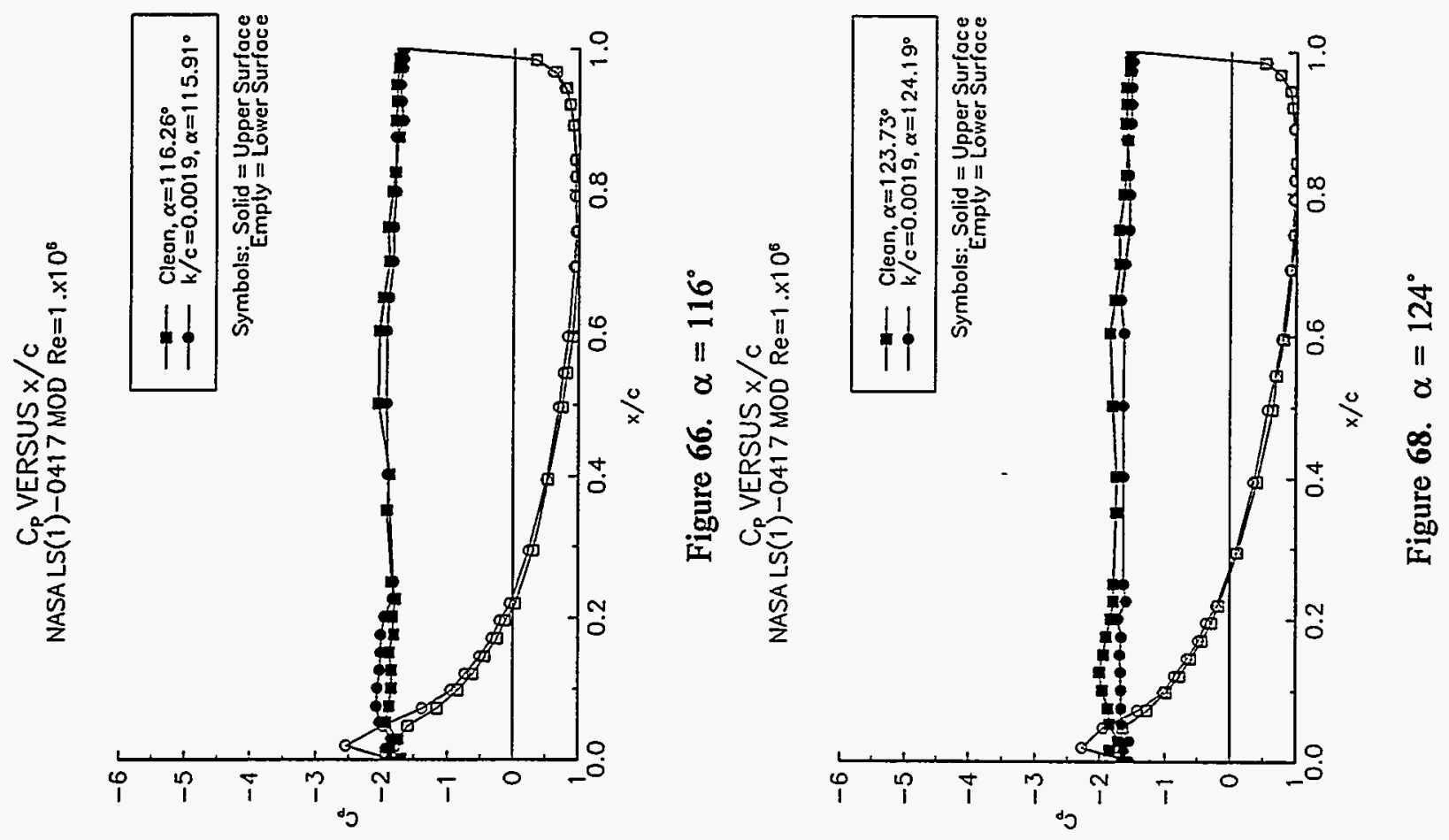

离
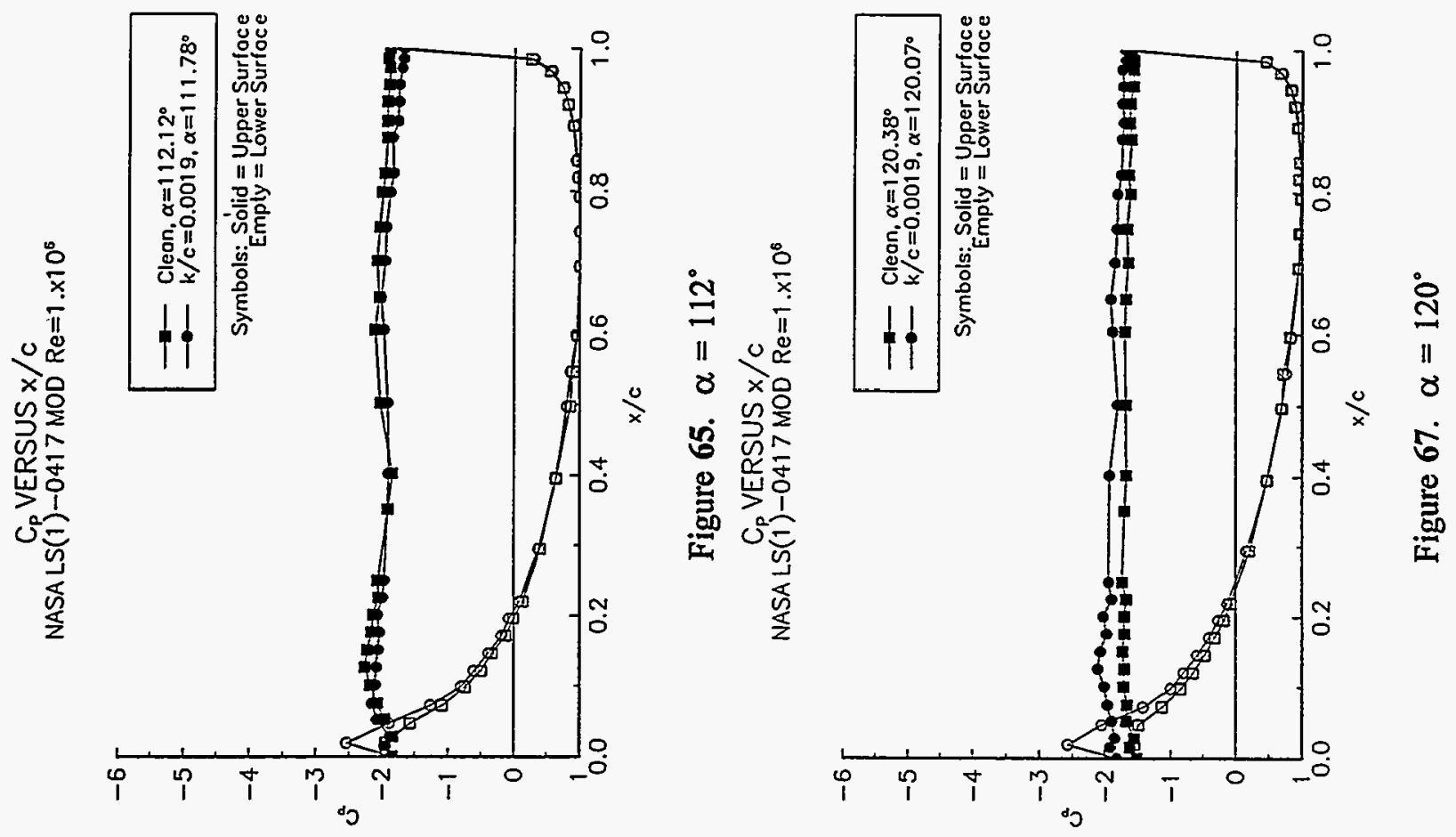
$C_{\mathrm{p}}$ VERSUS $\mathrm{x} / \mathrm{C}$

NASALS(1) -0417 MOD $R e=1 . \times 10^{6}$

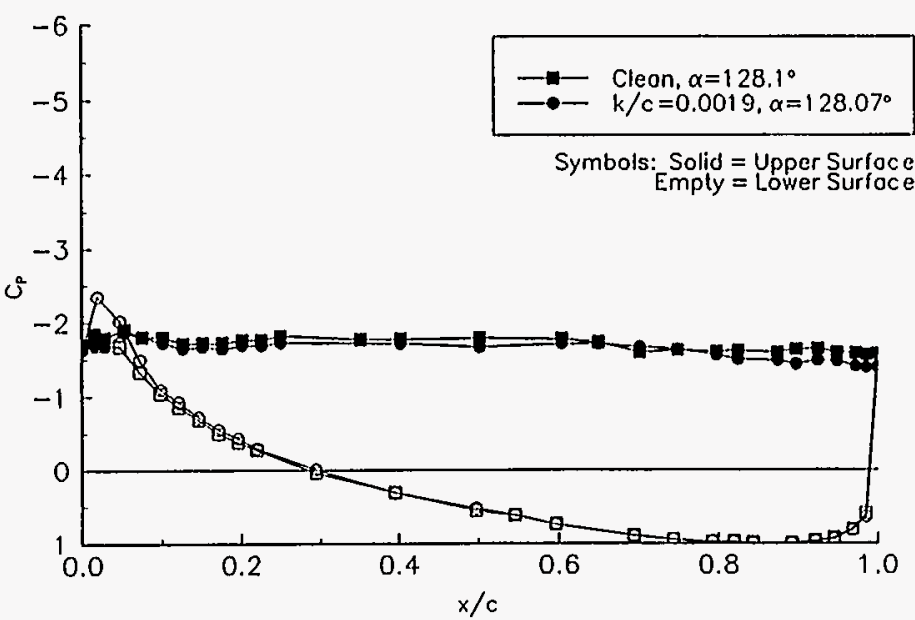

Figure 69. $\alpha=128^{\circ}$

$C_{p}$ VERSUS $x / c$

NASA LS(1) -0417 MOD $R e=1 . \times 10^{\circ}$

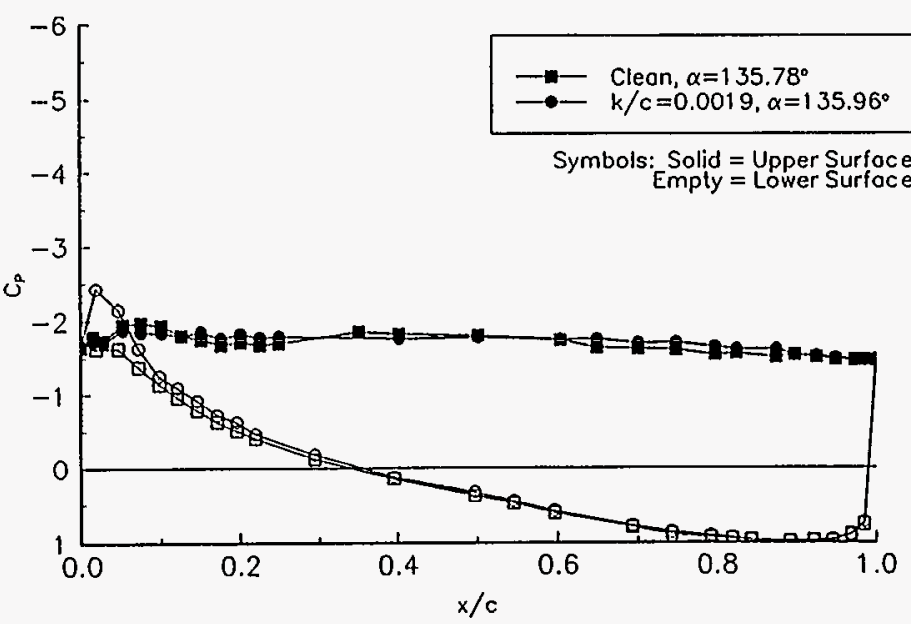

Figure 71. $\alpha=136^{\circ}$
$\mathrm{C}_{\mathrm{p}}$ VERSUS $\mathrm{x} / \mathrm{C}$

NASA LS(1)-0417 MOD $R e=1 . \times 10^{\circ}$

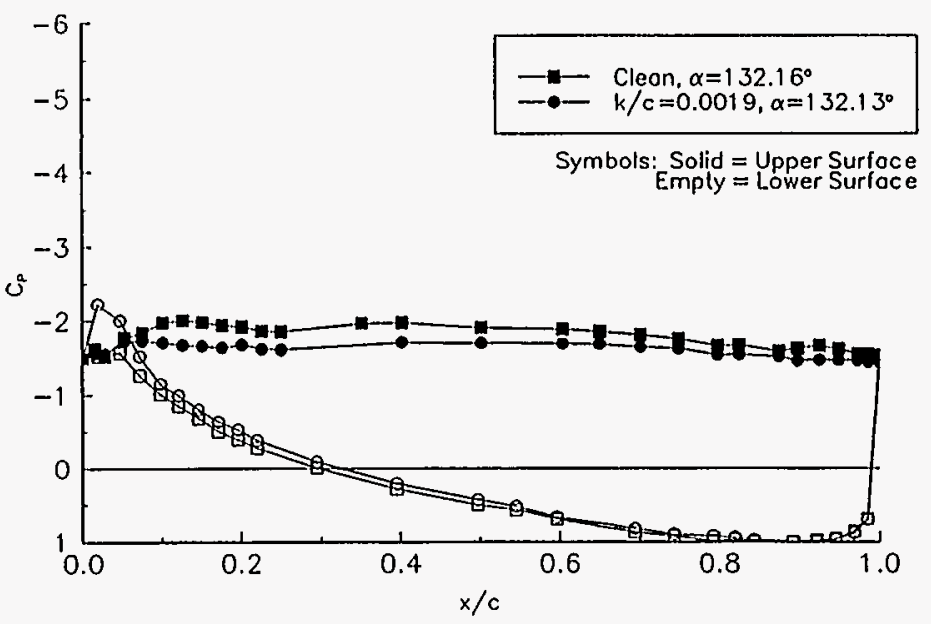

Figure 70. $\alpha=132^{\circ}$

$C_{p}$ VERSUS $x / c$

NASA LS(1) -0417 MOD $R e=1 . \times 10^{\circ}$

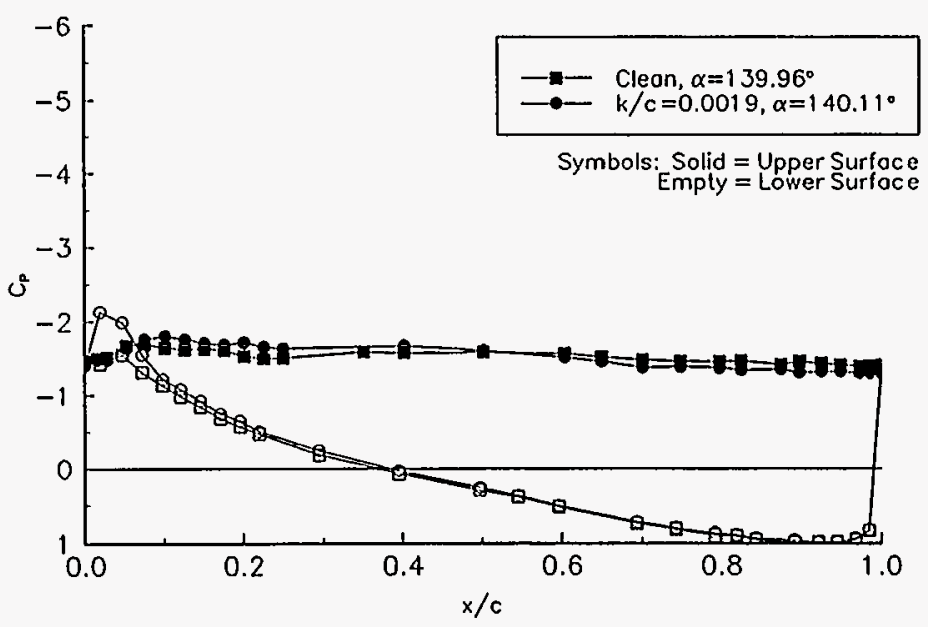

Figure 72. $\alpha=140^{\circ}$ 
$C_{p}$ VERSUS $x / c$

NASA LS(1) -0417 MOD Re $=1 . \times 10^{6}$

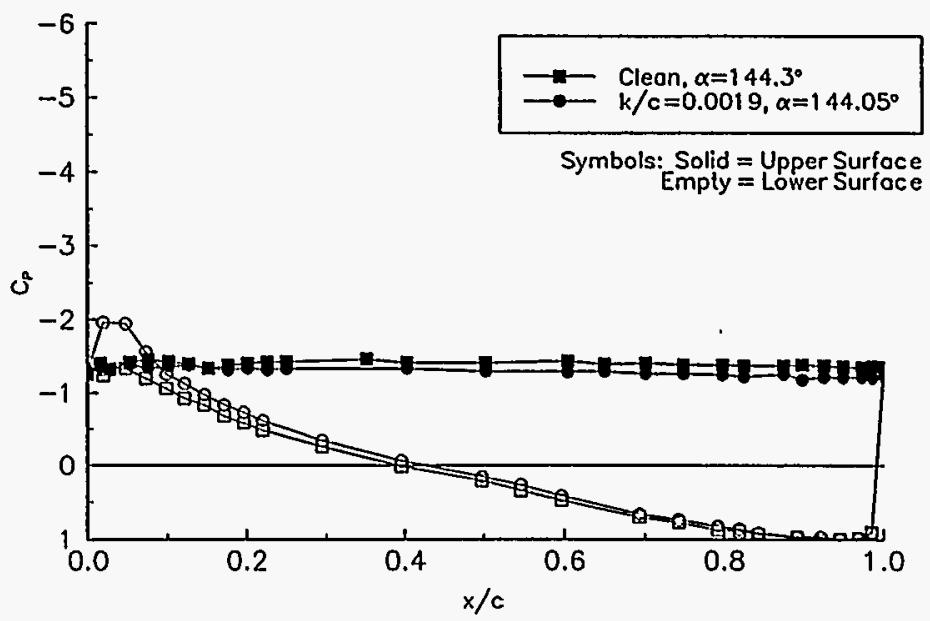

Figure 73. $\alpha=144^{\circ}$

$C_{p}$ VERSUS $x / c$

NASA LS(1) -0417 MOD $R e=1 . \times 10^{6}$

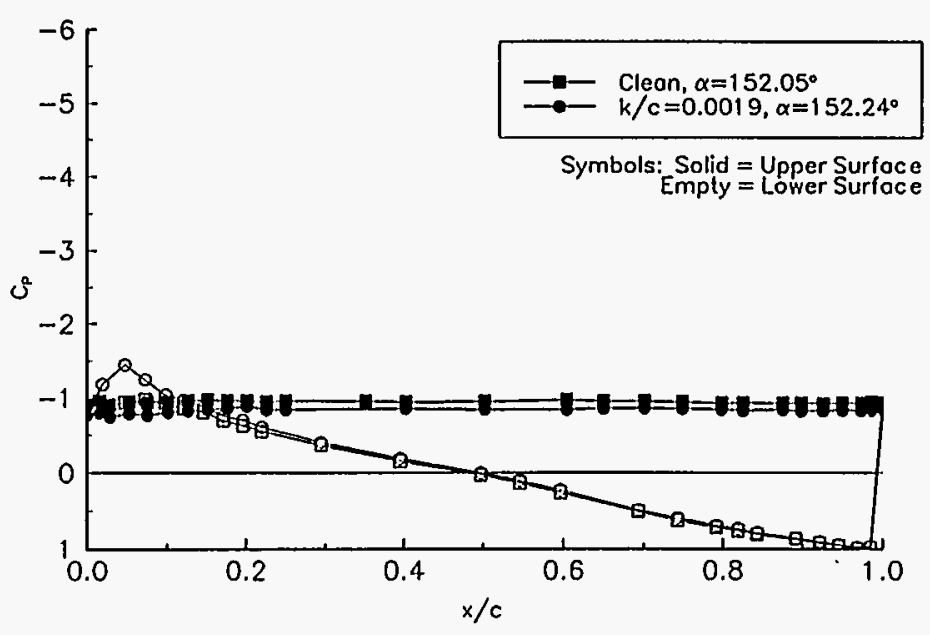

Figure 75. $\alpha=152^{\circ}$
$C_{p}$ VERSUS $x / c$

NASA LS(1) -0417 MOD $R e=1 . \times 10^{\circ}$

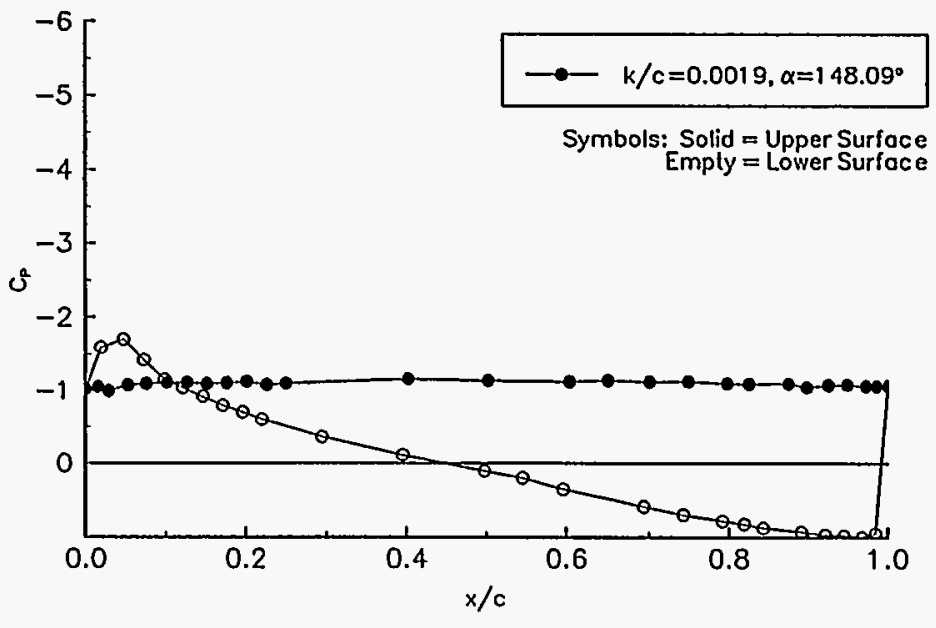

Figure 74. $\alpha=148^{\circ}$

$C_{p}$ VERSUS $x / c$

NASA LS(1) -0417 MOD Re $=1 . \times 10^{6}$

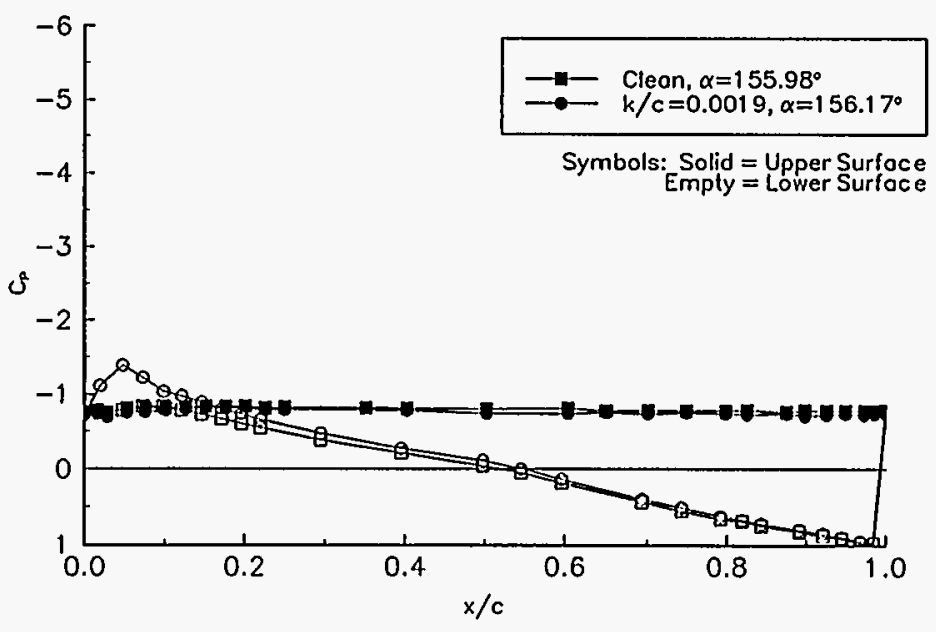

Figure 76. $\alpha=156^{\circ}$ 
$C_{p}$ VERSUS $x / c$

NASA LS(1)-0417 MOD $R e=1 . \times 10^{6}$

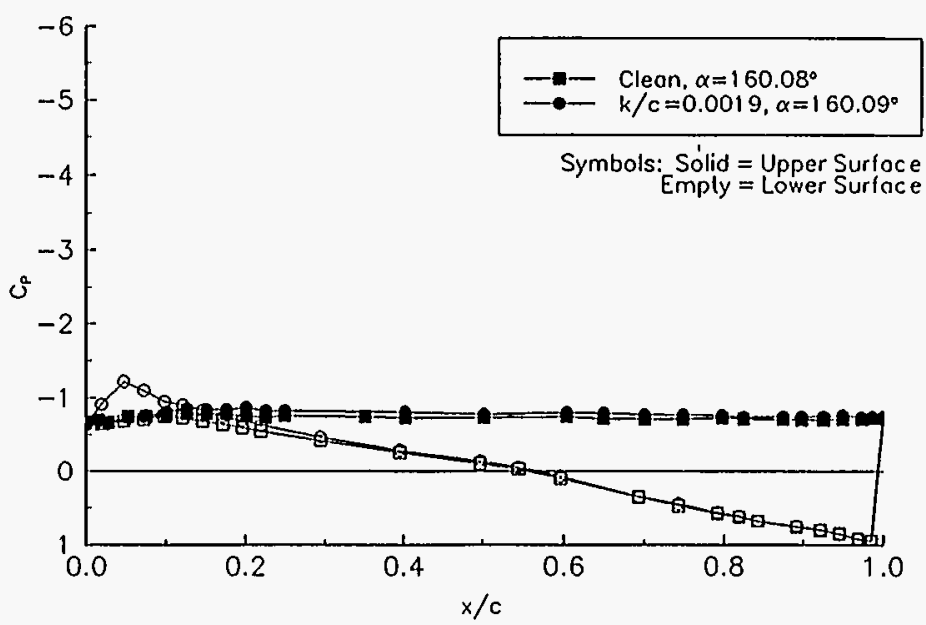

Figure 77. $\alpha=160^{\circ}$

$C_{p}$ VERSUS $x / c$

NASA LS (1) -0417 MOD $\operatorname{Re}=1 . \times 10^{\circ}$

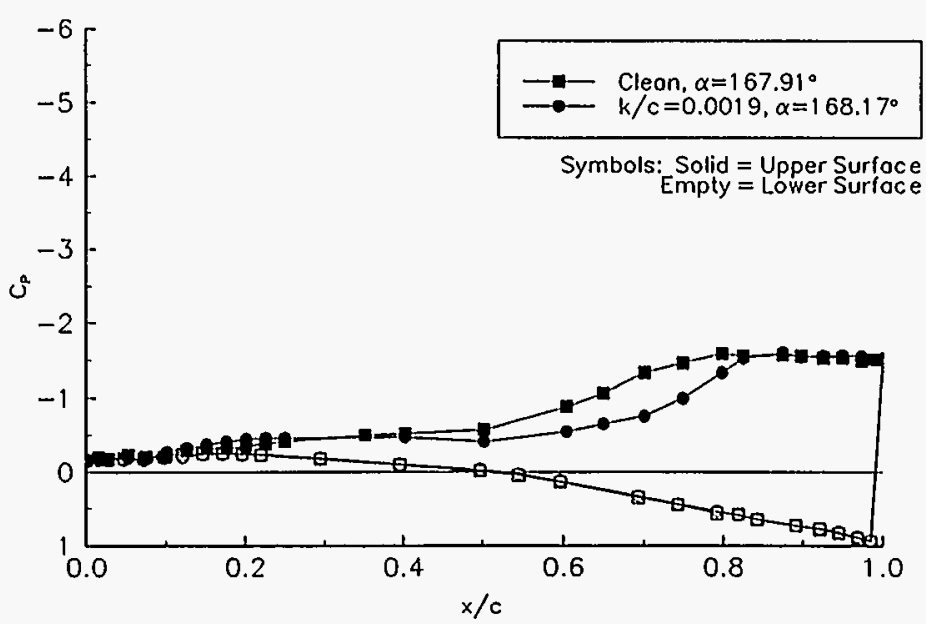

Figure 79. $\alpha=168^{\circ}$
$C_{p}$ VERSUS $x / c$

NASA LS(1)-0417 MOD Re $=1 \times 10^{\circ}$

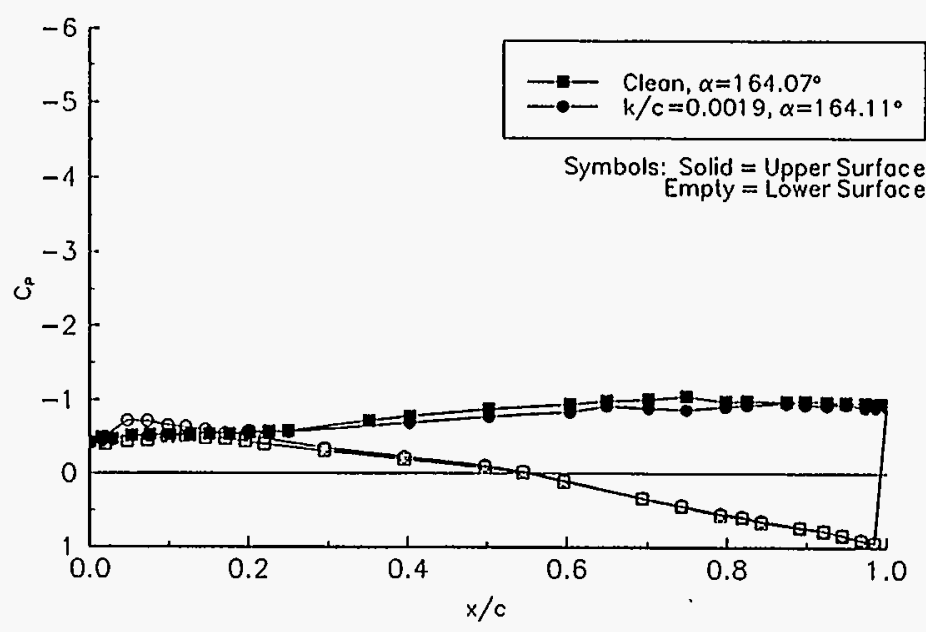

Figure 78. $\alpha=164^{\circ}$

$C_{p}$ VERSUS $x / c$

NASA LS(1)-0417 MOD Re $=1 . \times 10^{\circ}$

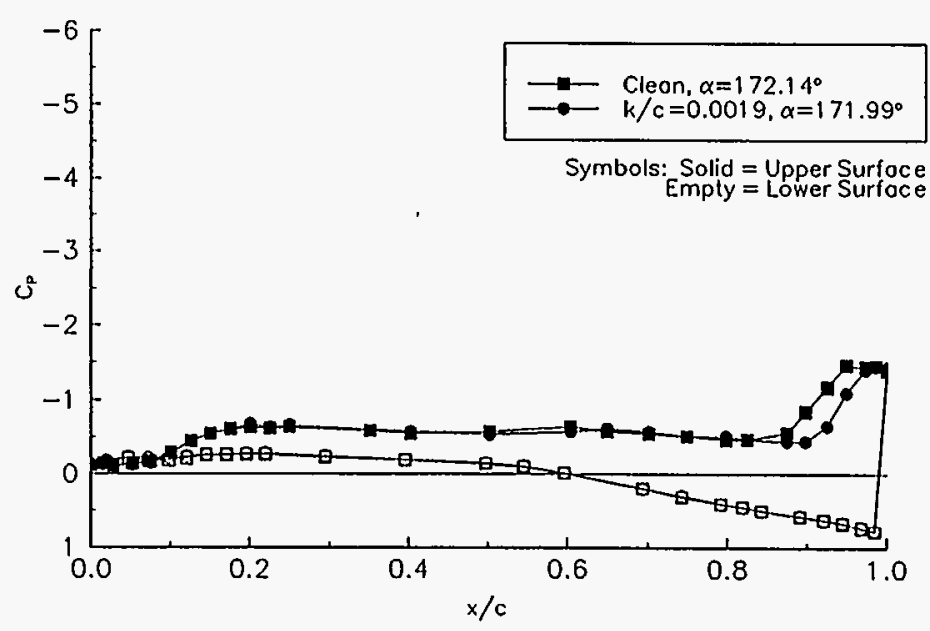

Figure 80. $\alpha=172^{\circ}$ 
$C_{p}$ VERSUS $x / C$

NASA LS(1) -0417 MOD Re $=1 . \times 10^{6}$

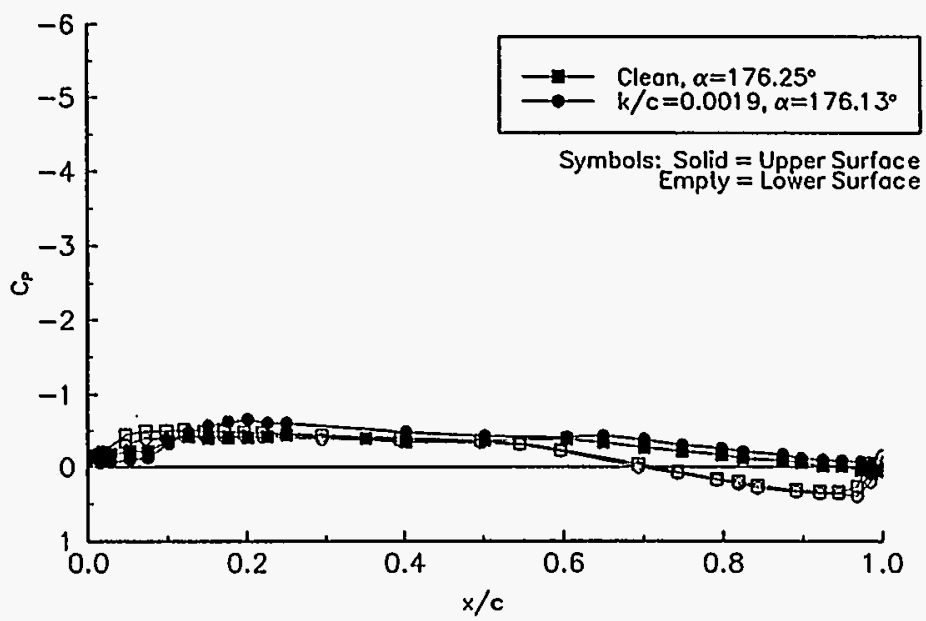

Figure 81. $\alpha=176^{\circ}$

$\mathrm{C}_{\mathrm{p}}$ VERSUS $\mathrm{x} / \mathrm{c}$

NASA LS(1) -0417 MOD $R e=1 . \times 10^{\circ}$

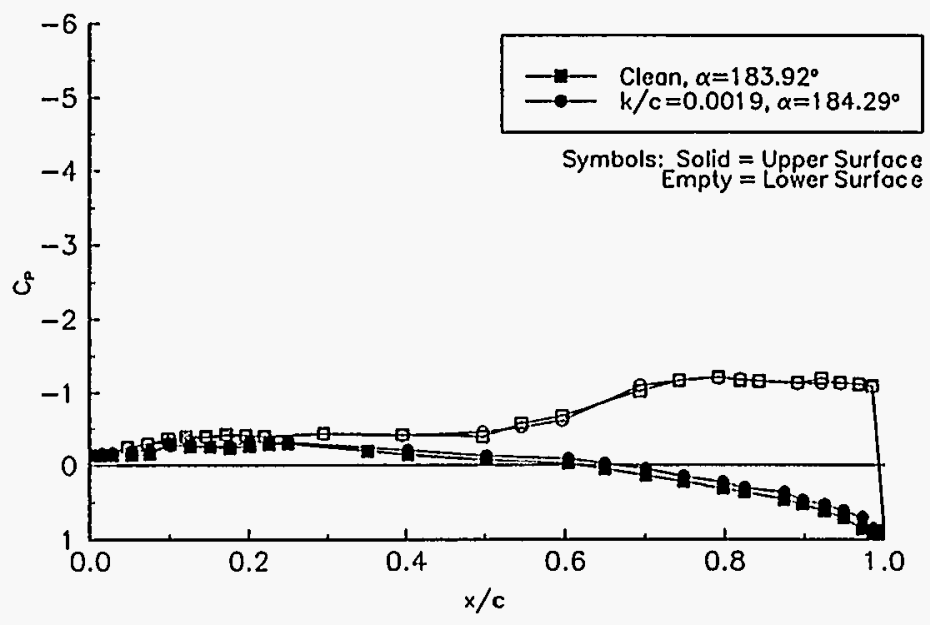

Figure 83. $\alpha=184^{\circ}$
$C_{p}$ VERSUS $x / c$

NASA LS(1) -0417 MOD $R e=1 . \times 10^{6}$

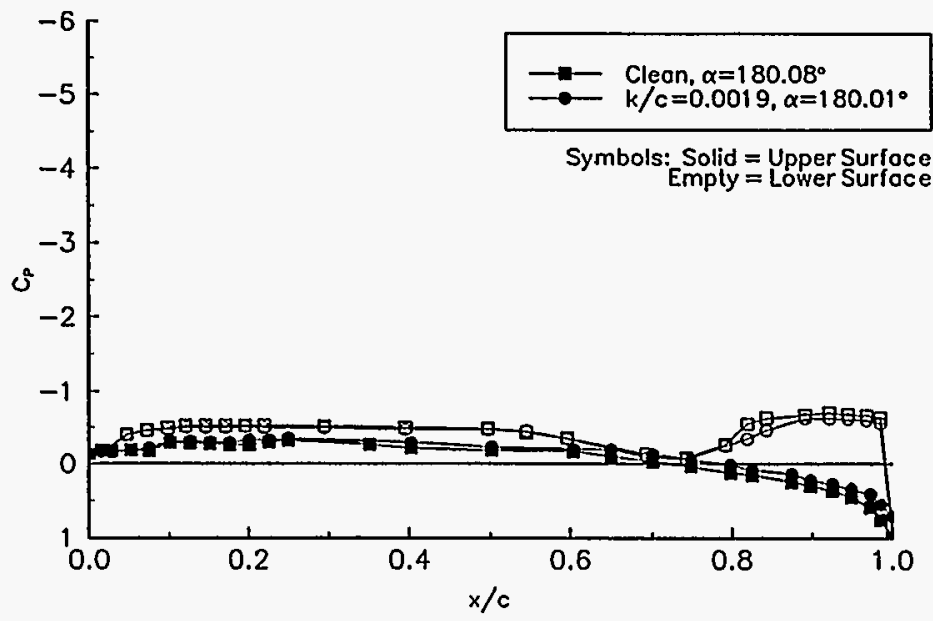

Figure 82. $\alpha=180^{\circ}$

$\mathrm{C}_{\mathrm{p}}$ VERSUS $\mathrm{x} / \mathrm{c}$

NASA LS(1) -0417 MOO $R e=1 . \times 10^{\circ}$

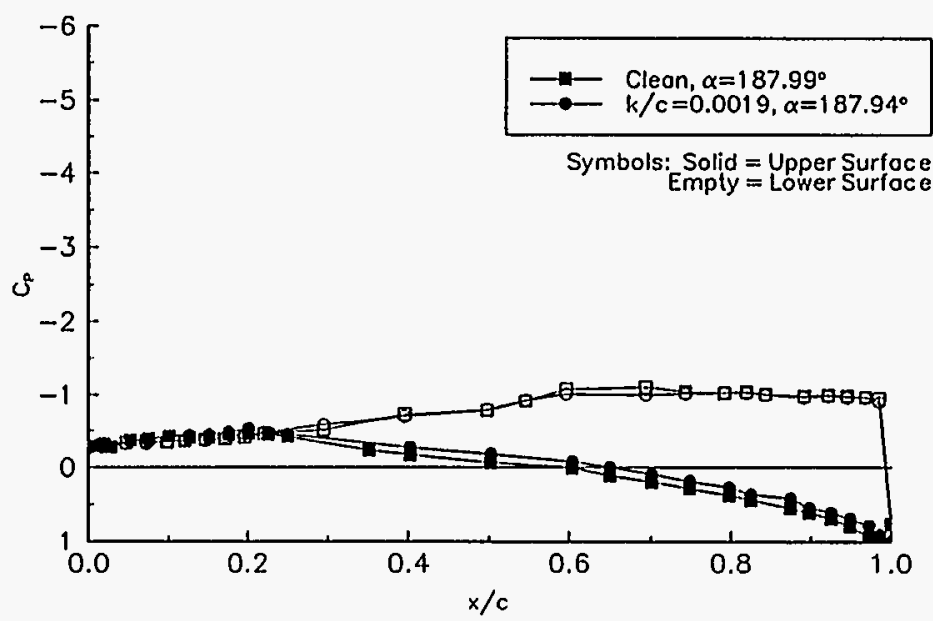

Figure 84. $\alpha=188^{\circ}$ 
$C_{p}$ VERSUS $x / c$

NASA LS(1)-0417 MOD Re $=1 . \times 10^{6}$

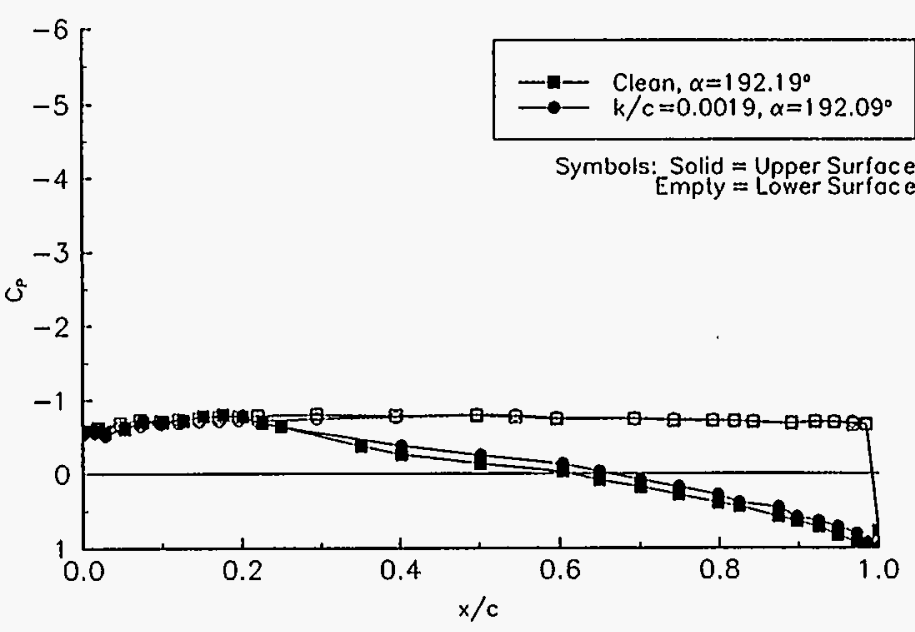

Figure 85. $\alpha=192^{\circ}$

$C_{P}$ VERSUS $x$

NASA LS(1) $-0417 \mathrm{MOD} \operatorname{Re}=1 . \times 10^{\circ}$

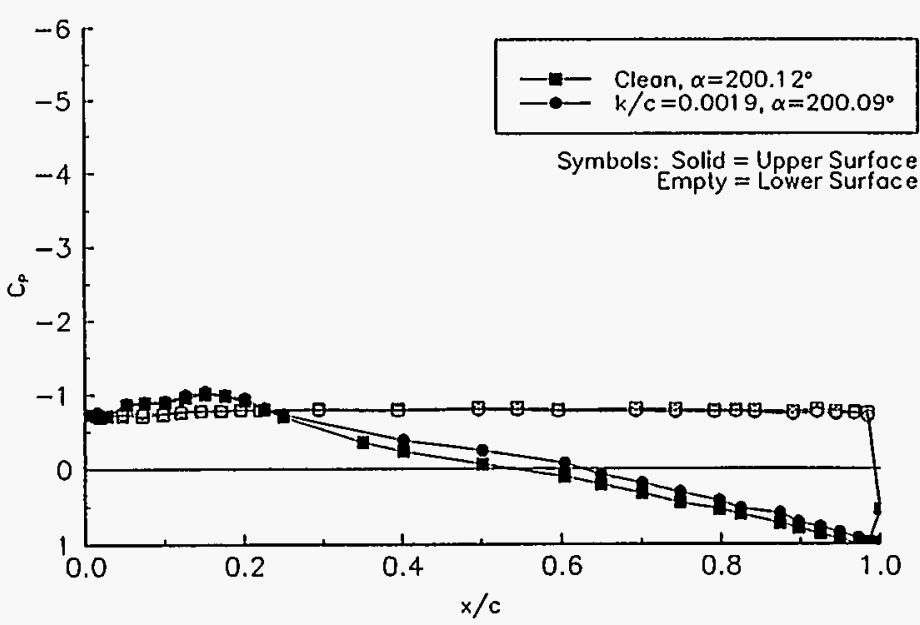

Figure 87. $\alpha=200^{\circ}$
$C_{p}$ VERSUS $x / C$

NASA LS(1)-0417 MOD $R e=1 . \times 10^{\circ}$

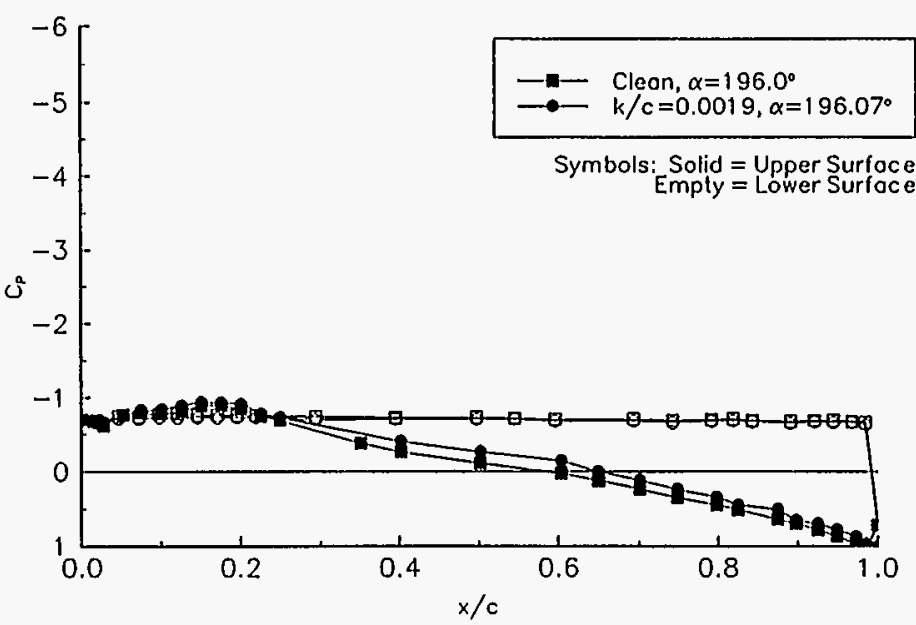

Figure 86. $\alpha=196^{\circ}$

$C_{p}$ VERSUS $x / c$

NASA LS(1)-0417 MOD $R e=1 . \times 10^{\circ}$

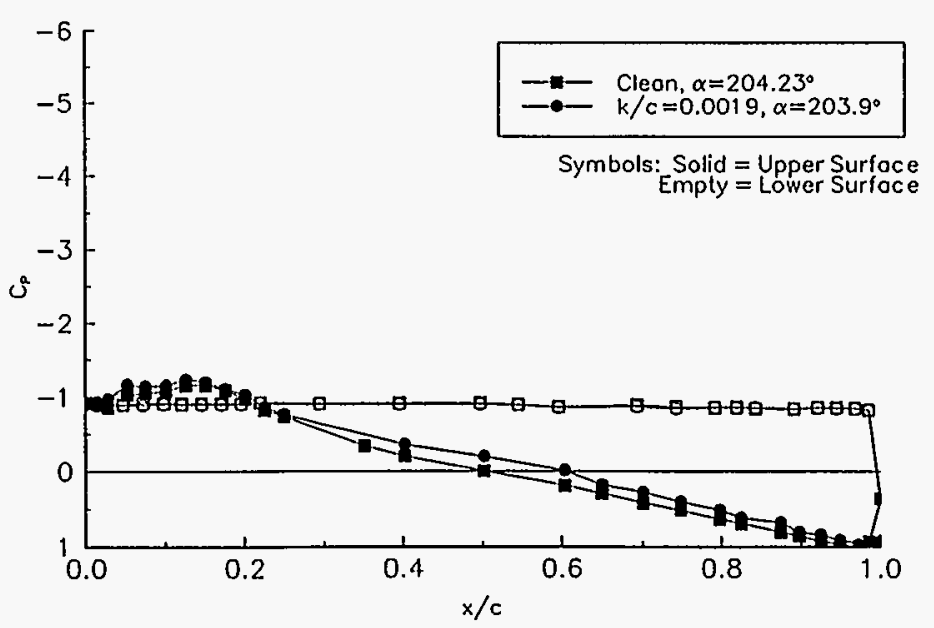

Figure 88. $\alpha=204^{\circ}$ 
$C_{\mathrm{p}}$ VERSUS $X / \mathrm{c}$

NASA LS (1) -0417 MOD Re $=1 . \times 10^{6}$

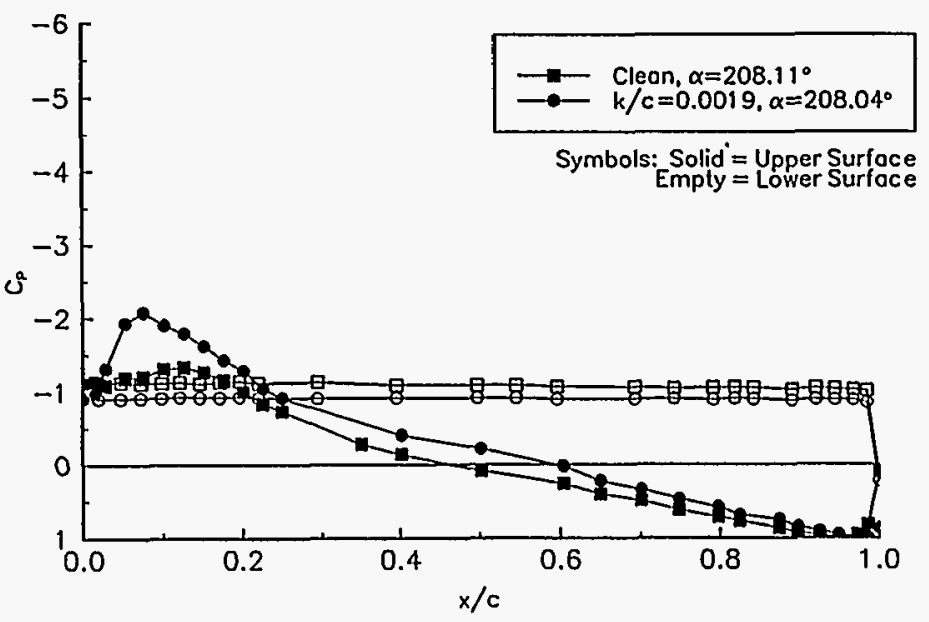

Figure 89. $\alpha=208^{\circ}$

$C_{p}$ VERSUS $x / c$

NASA LS (1) -0417 MOD $R e=1 . \times 10^{8}$

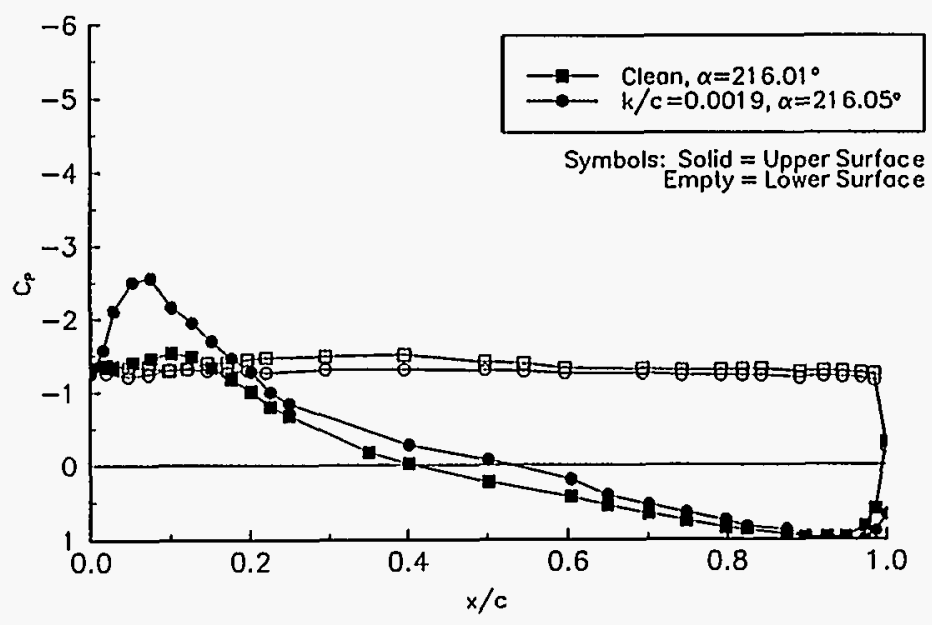

Figure 91. $\alpha=216^{\circ}$
$C_{p}$ VERSUS $x / c$

NASA LS (1)-0417 MOD Re $=1 . \times 10^{6}$

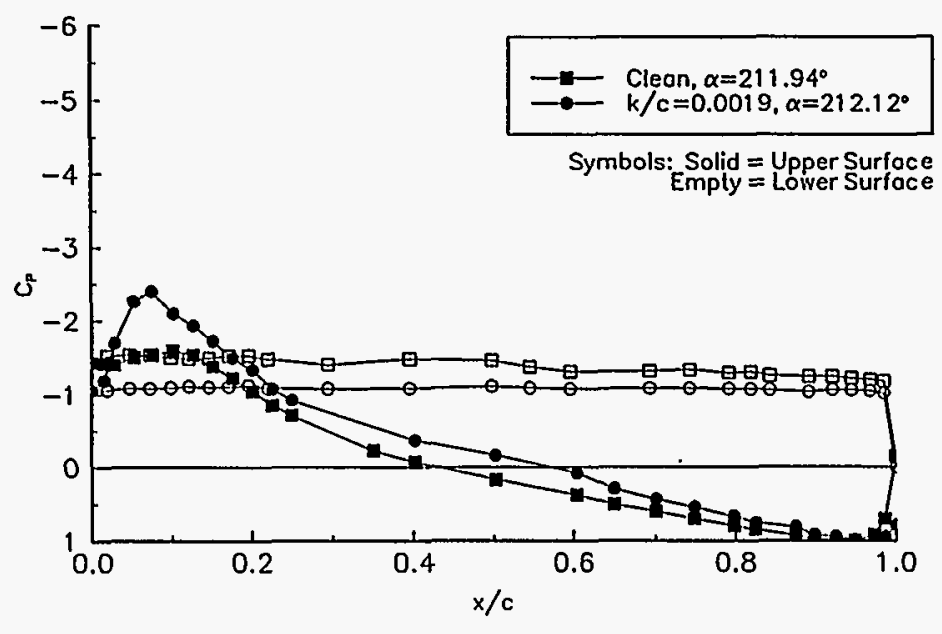

Figure 90. $\alpha=212^{\circ}$

$C_{p}$ VERSUS $x / c$ NASA LS (1) -0417 MOD Re $=1 . \times 10^{\circ}$

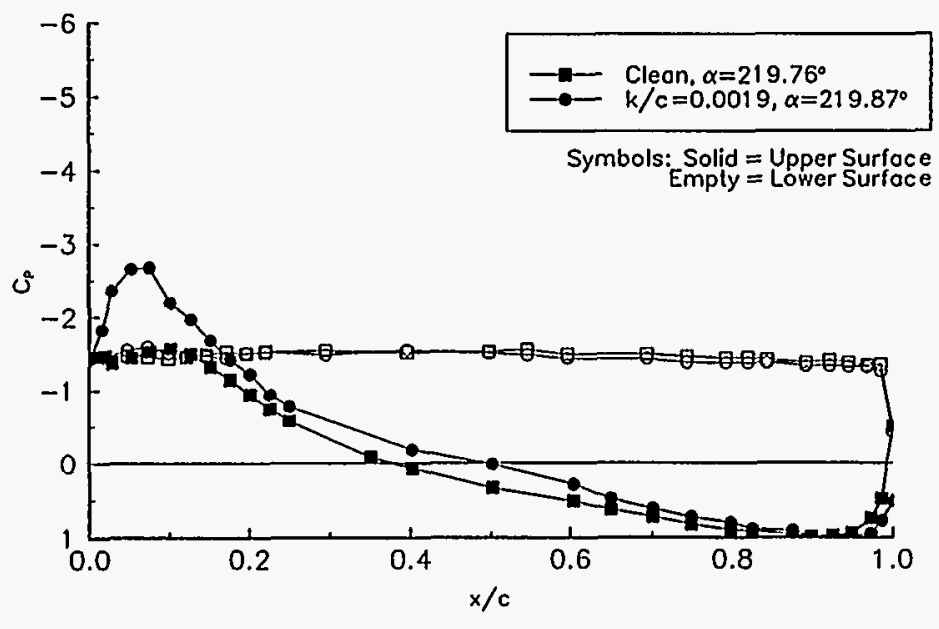

Figure 92. $\alpha=220^{\circ}$ 
$C_{p}$ VERSUS $x / \mathrm{C}$

NASALS(1) -0417 MOD $R e=1 . \times 10^{\circ}$

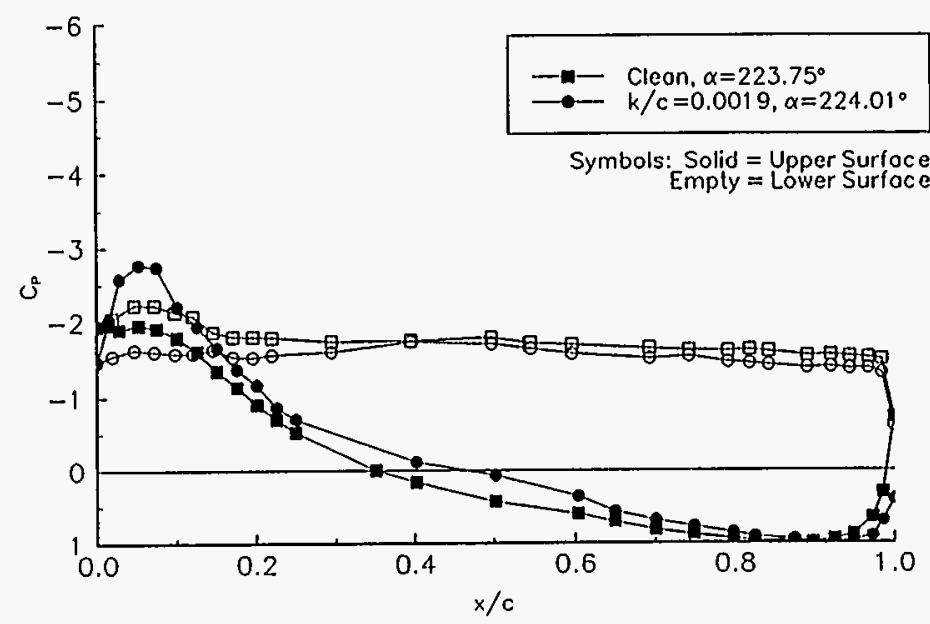

Figure 93. $\alpha=224^{\circ}$

$C_{P}$ VERSUS $x / C$
NASA LS(1) -0417 MOD Re $=1 . \times 10^{\circ}$

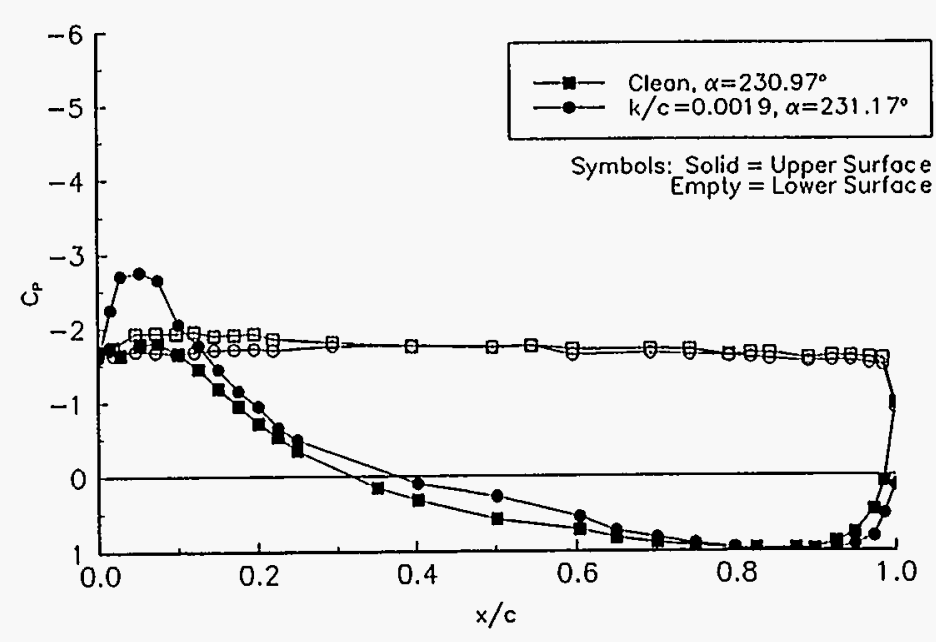

Figure 95. $\alpha=231^{\circ}$
$C_{p}$ VERSUS $x / c$

NASALS(1)-0417 MOD Re $=1 . \times 10^{\circ}$

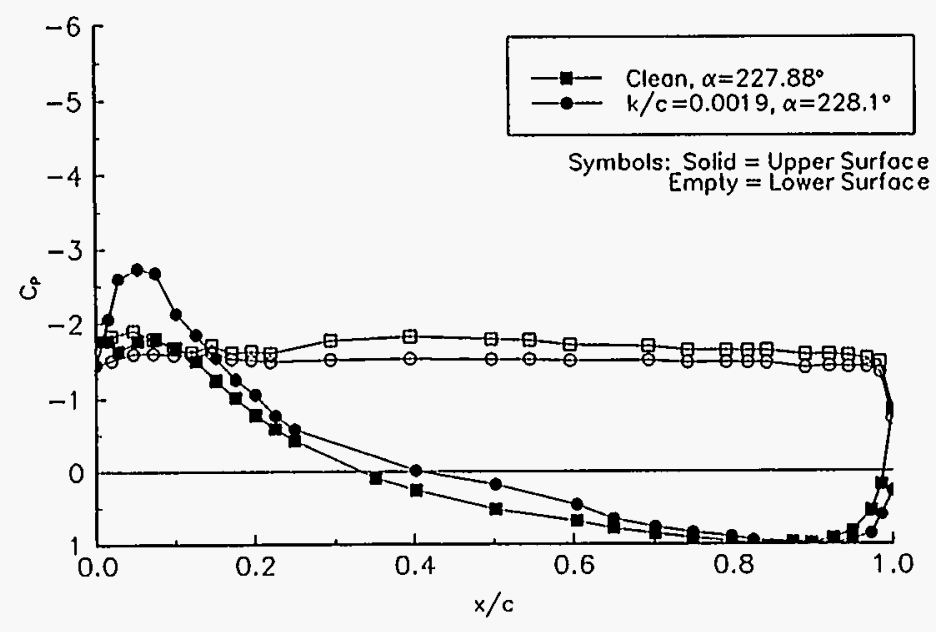

Figure 94. $\alpha=228^{\circ}$ 


\section{Steady State Pressure Distributions}

$$
\text { VGs, } R e=1.0 \text { million }
$$


$C_{p}$ VERSUS $x / c$

NASA LS $(1)-0417$ MOO $\left(R_{c}=1.0 \times 10^{6}\right)$

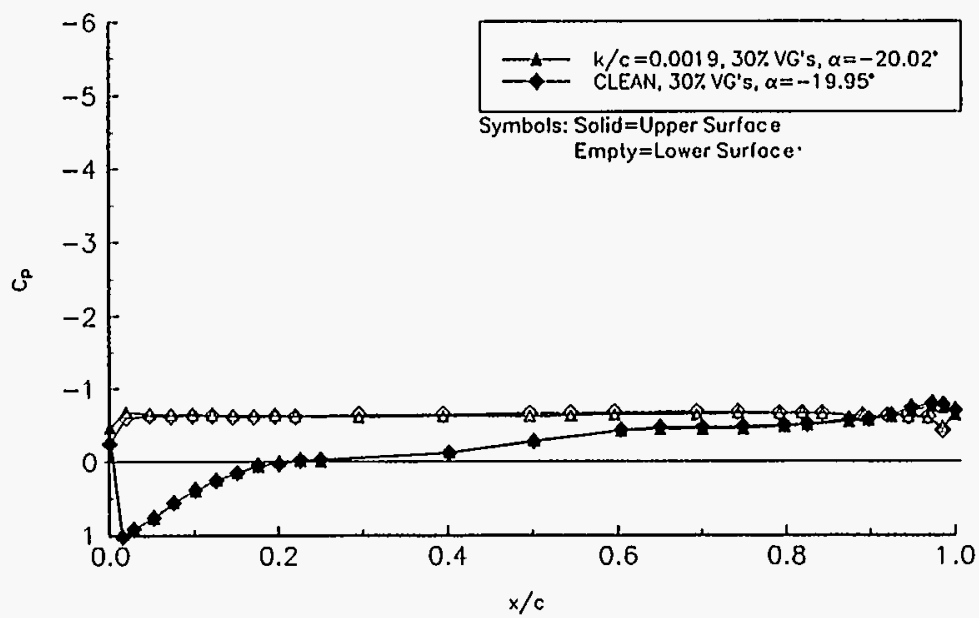

Figure 96. $\alpha=-20^{\circ}$

$C_{p}$ VERSUS $x / c$

NASA LS(1)-0417 MOD $\left(R_{\mathbf{e}}=1.0 \times 10^{6}\right)$

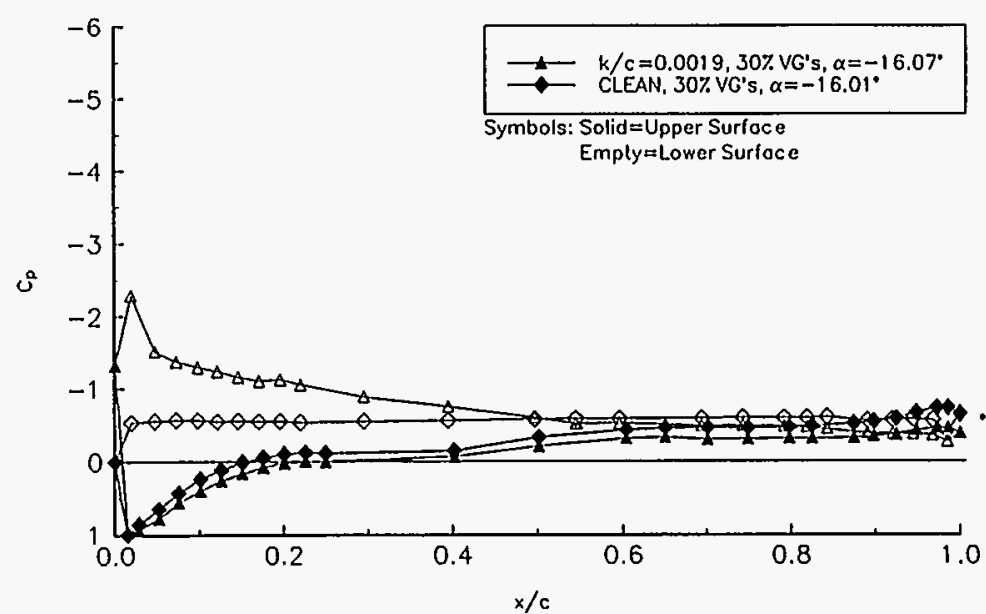

Figure 98. $\alpha=-16^{\circ}$
$\mathrm{C}_{\rho}$ VERSUS $\mathrm{x} / \mathrm{c}$

NASA LS(1)-0417 MOO $\left(R_{0}=1.0 \times 10^{\circ}\right)$

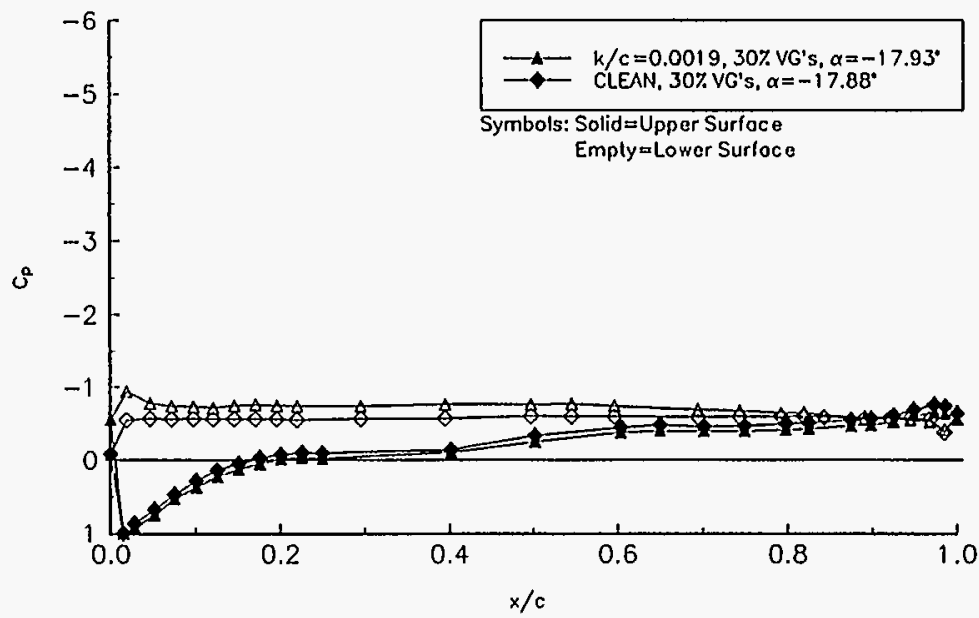

Figure 97. $\alpha=-18^{\circ}$

$C_{p}$ VERSUS $x / c$

NASA LS $(1)-0417$ MOD $\left(R_{e}=1.0 \times 10^{6}\right)$

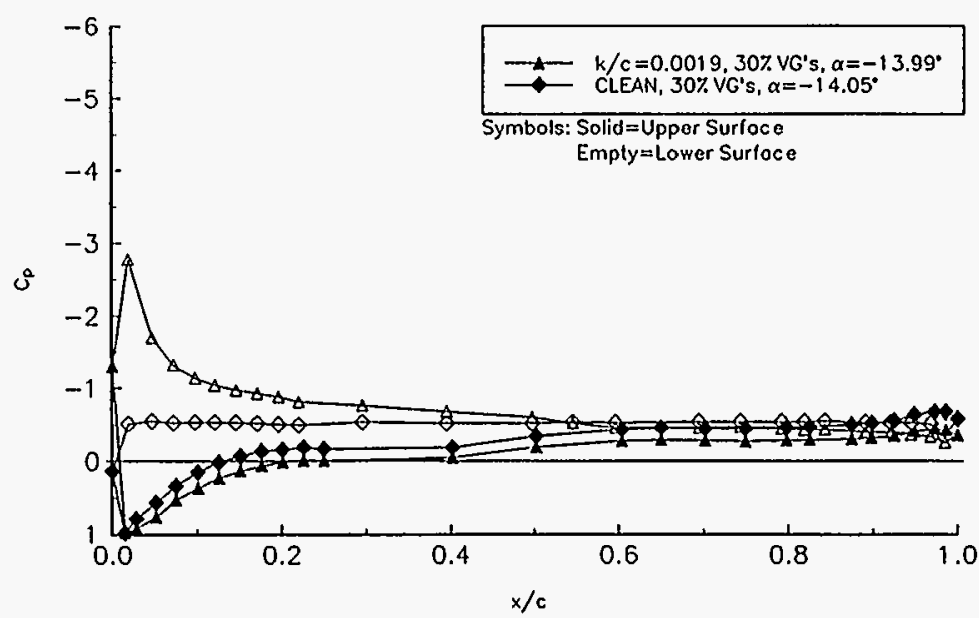

Figure 99. $\alpha=-14^{\circ}$ 
$C_{p}$ VERSUS $x / c$

NASA LS(1)-0417MOD $\left(R_{B}=1.0 \times 10^{6}\right)$

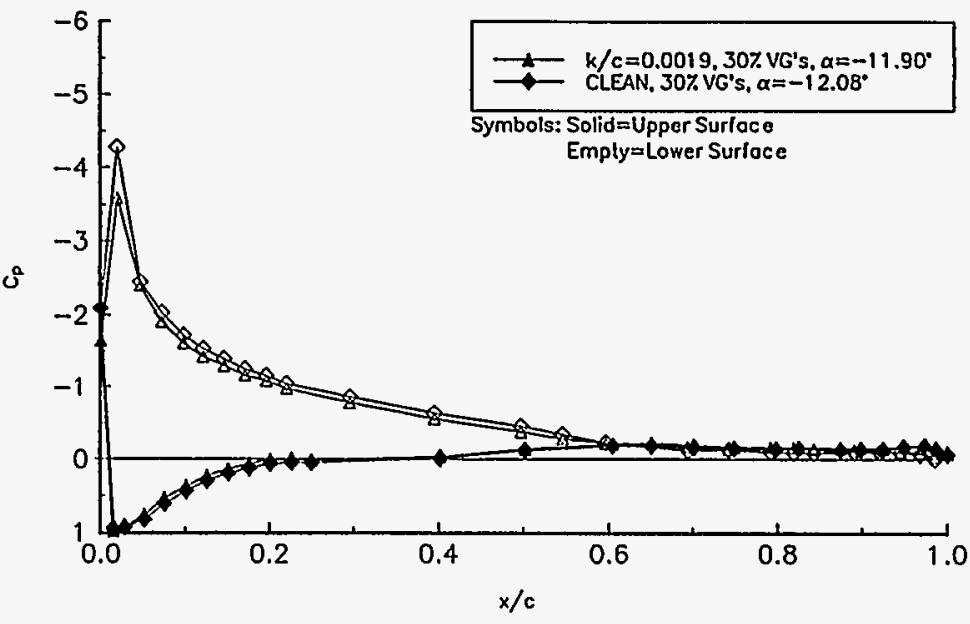

Figure 100. $\alpha=-12^{\circ}$

$\mathrm{C}_{\mathrm{p}}$ VERSUS $\times / \mathrm{c}$

NASA LS(1) $-0417 \mathrm{MOD}\left(R_{\varepsilon}=1.0 \times 10^{6}\right)$

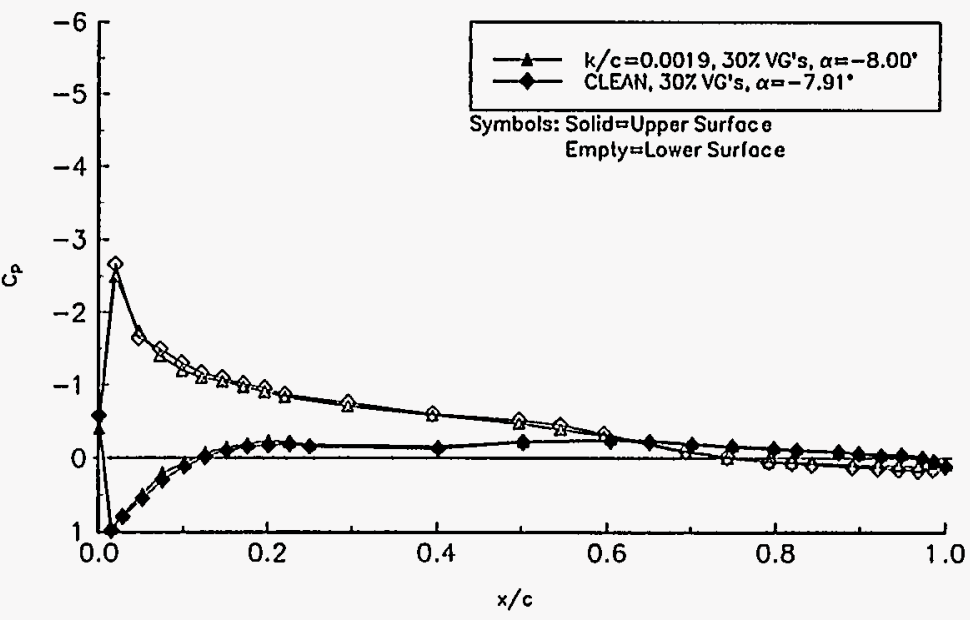

Figure 102. $\alpha=-8^{\circ}$
$C_{p}$ VERSUS $x / c$

NASA LS(1) $-0417 \mathrm{MOD}\left(R_{e}=1.0 \times 10^{6}\right)$

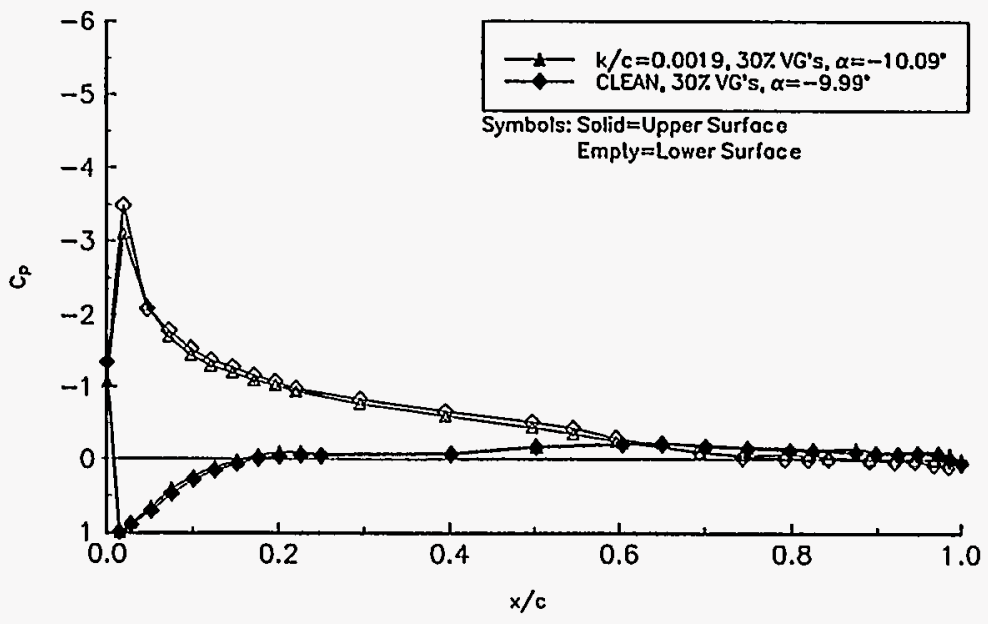

Figure 101. $\alpha=-10^{\circ}$

$C_{p}$ VERSUS $x / c$

NASA LS $(1)-0417 \mathrm{MOO}\left(R_{\mathrm{e}}=1.0 \times 10^{\circ}\right)$

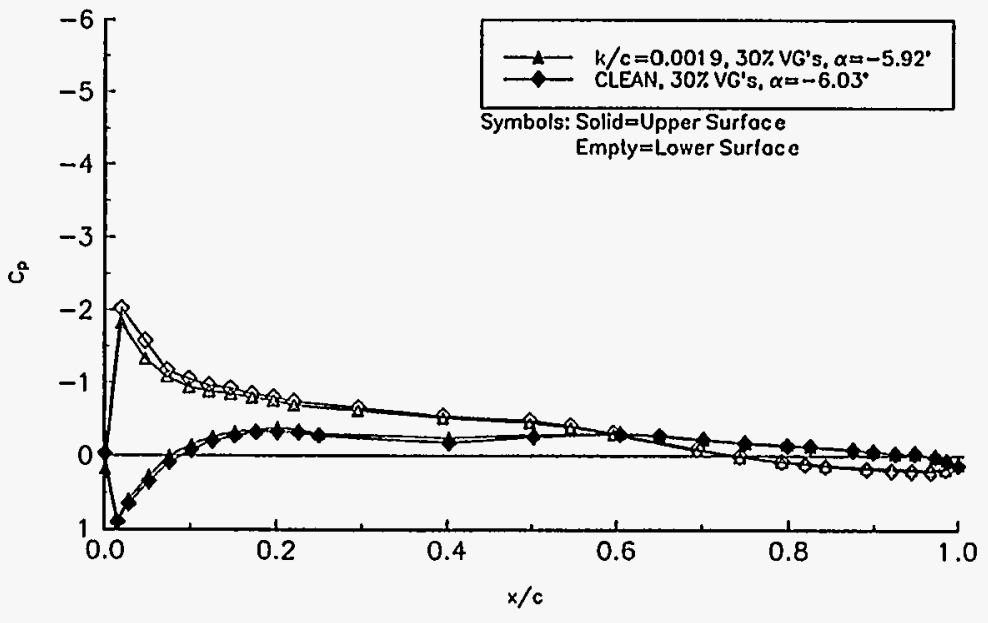

Figure 103. $\alpha=-6^{\circ}$ 
$\mathrm{C}_{\mathrm{p}}$ VERSUS $\mathrm{x} / \mathrm{C}$

NASA LS(1)-0417 MOD $\left(R_{e}=1.0 \times 10^{6}\right)$

i

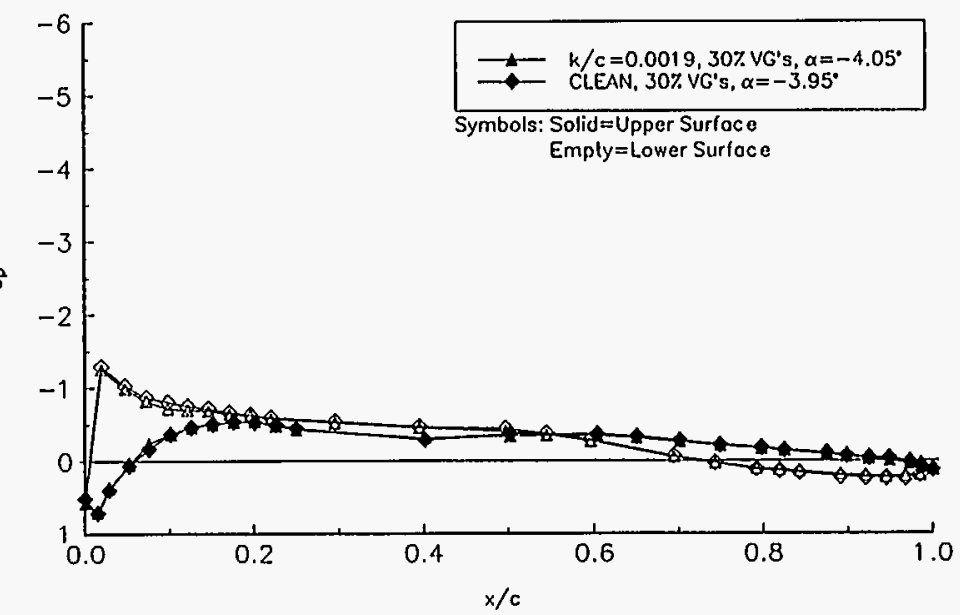

Figure 104. $\alpha=-4^{\circ}$

$C_{p}$ VERSUS $x / c$

NASA LS(1) $-0417 \mathrm{MOD}\left(R_{\mathbf{e}}=1.0 \times 10^{6}\right)$

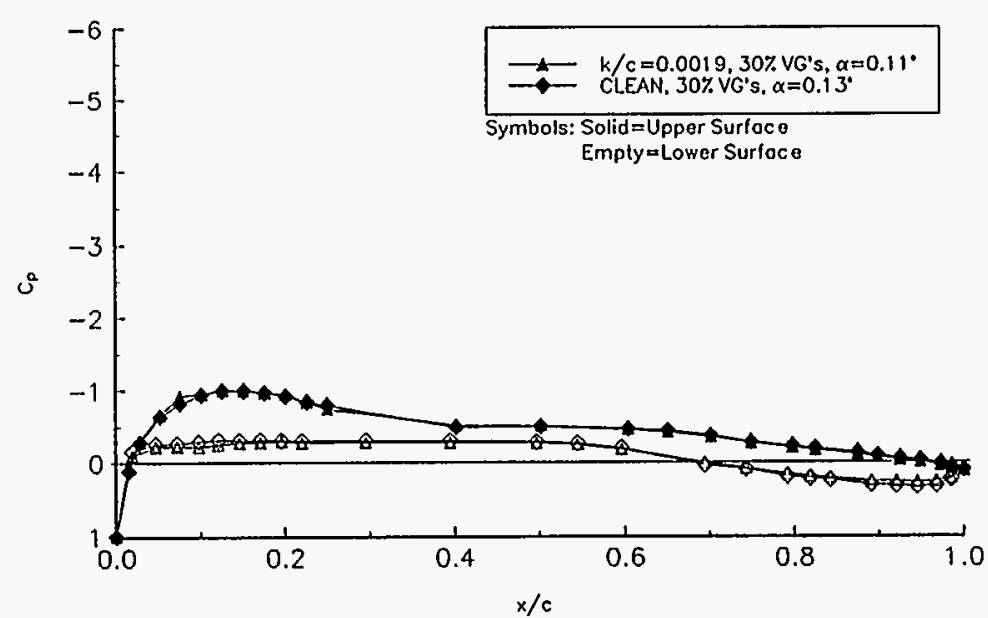

Figure 106. $\alpha=0^{\circ}$
$C_{p}$ VERSUS $x / c$

NASA LS (1) $-0417 \mathrm{MOD}\left(\mathrm{R}_{\mathrm{e}}=1.0 \times 10^{6}\right)$

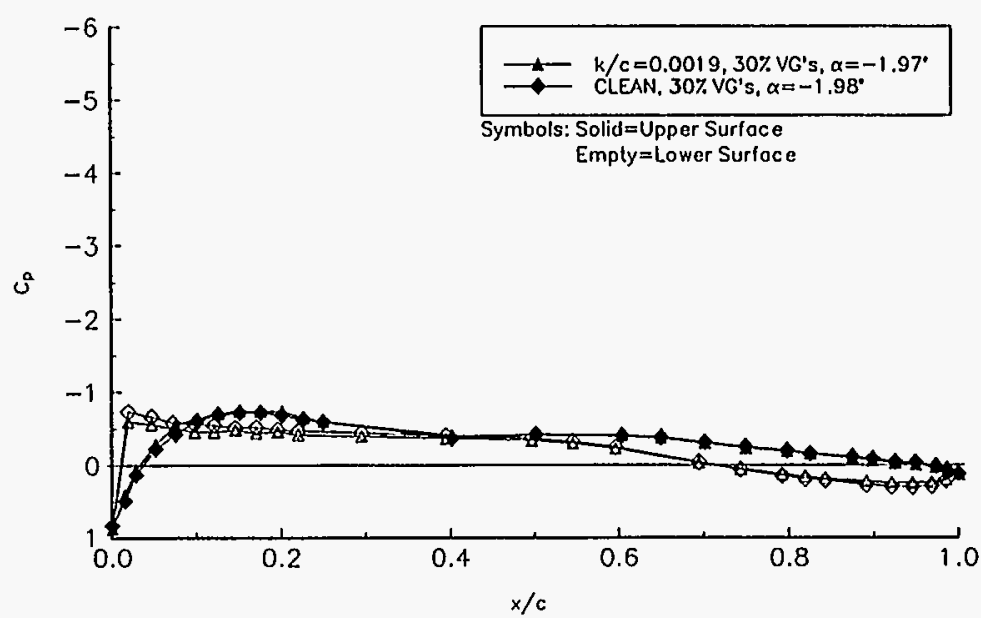

Figure 105. $\alpha=-2^{\circ}$

$C_{p}$ VERSUS $x / c$
NASA LS(1) - 0417 MOD $\left(R_{e}=1.0 \times 10^{6}\right)$

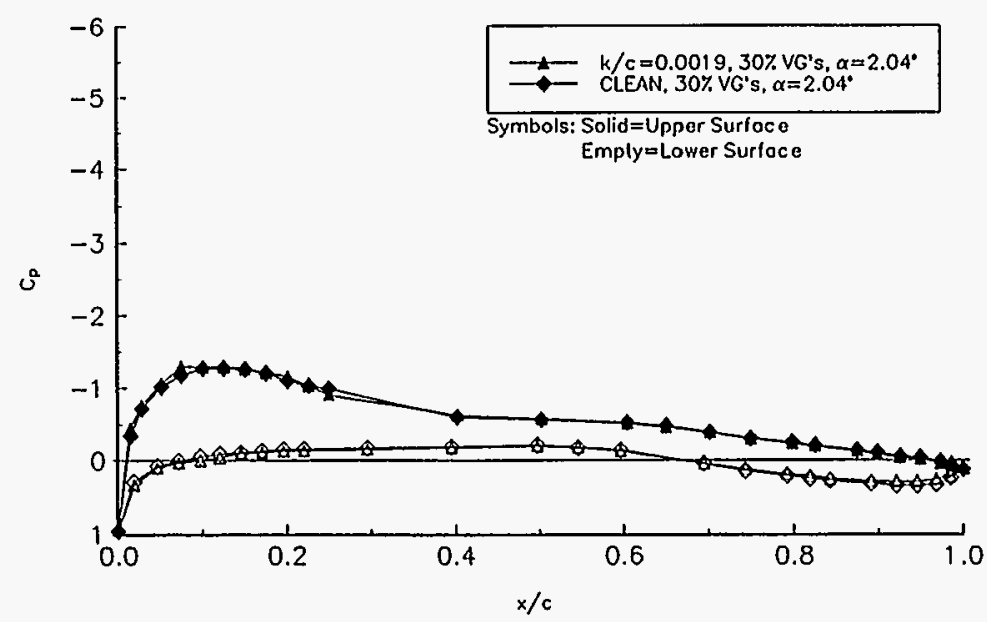

Figure 107. $\alpha=2^{\circ}$ 
$C_{\mathrm{p}}$ VERSUS $x / \mathrm{c}$

NASA LS(1) -0417 MOD $\left(R_{a}=1.0 \times 10^{\circ}\right)$

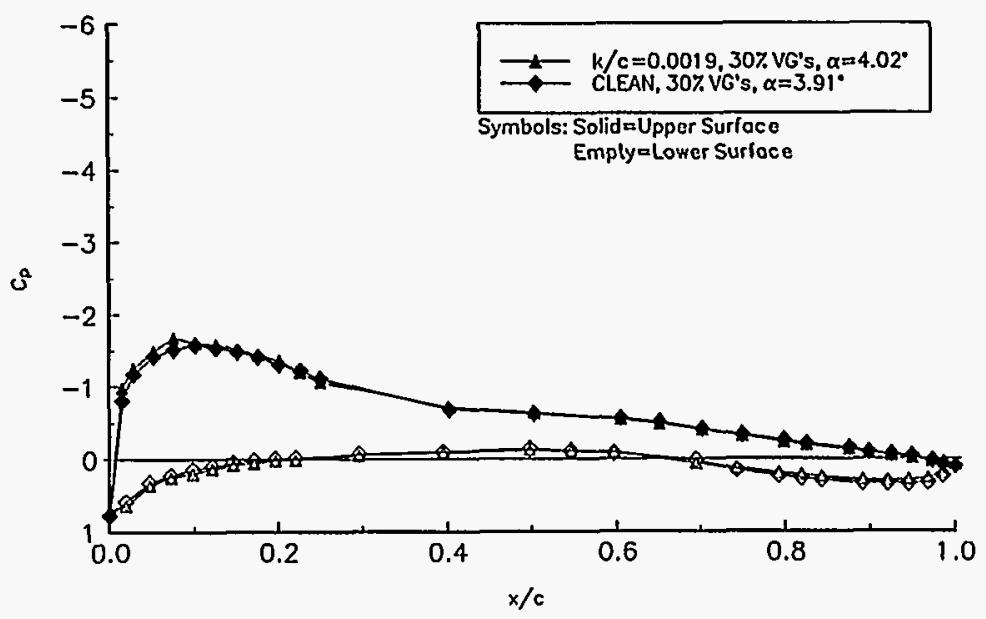

Figure 108. $\alpha=4^{\circ}$

$C_{p}$ VERSUS $x / c$

NASA LS(1)-0417 MOO $\left(R_{B}=1.0 \times 10^{6}\right)$

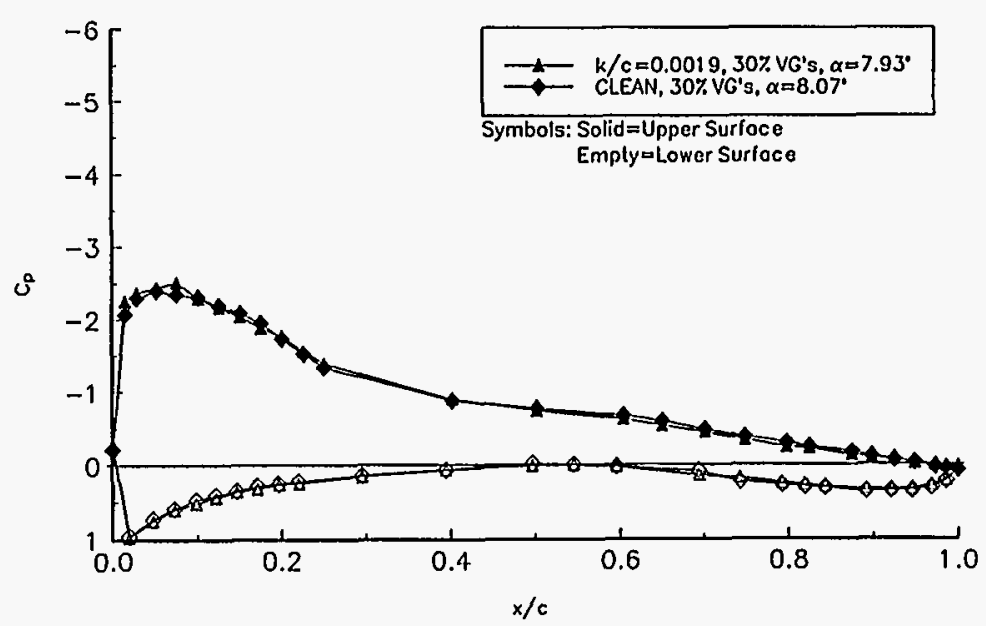

Figure 110. $\alpha=8^{\circ}$
$\mathrm{C}_{\rho}$ VERSUS $x / \mathrm{c}$

$\operatorname{NASALS}(1)-0417 \mathrm{MOD}\left(R_{\mathrm{B}}=1.0 \times 10^{\circ}\right)$

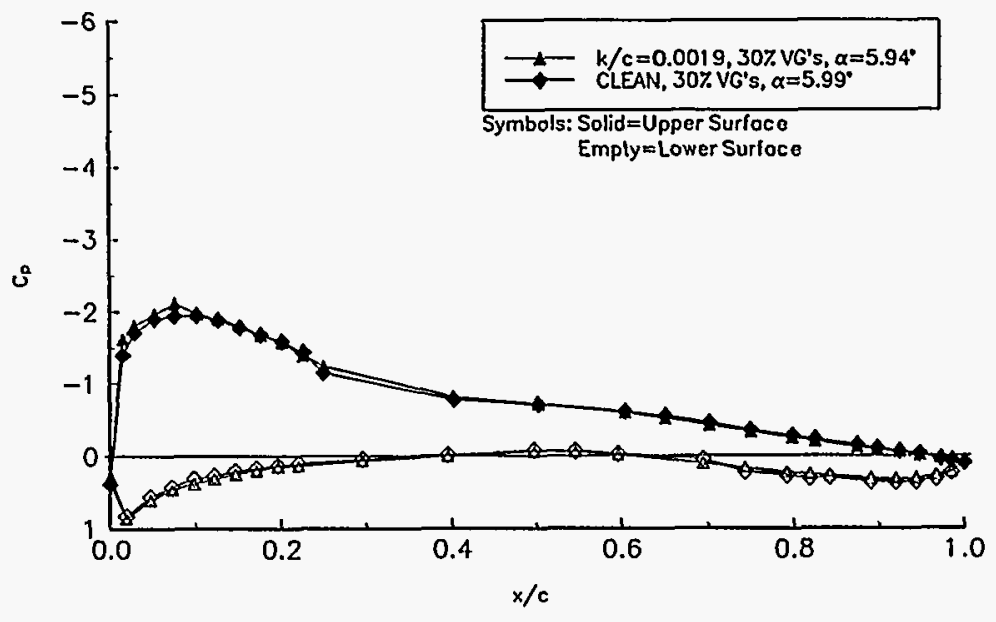

Figure 109. $\alpha=6^{\circ}$ $C_{p}$ VERSUS $x / c$
NASA LS 11$)-0417 \mathrm{MOO}\left(R_{0}=1.0 \times 10^{\circ}\right)$

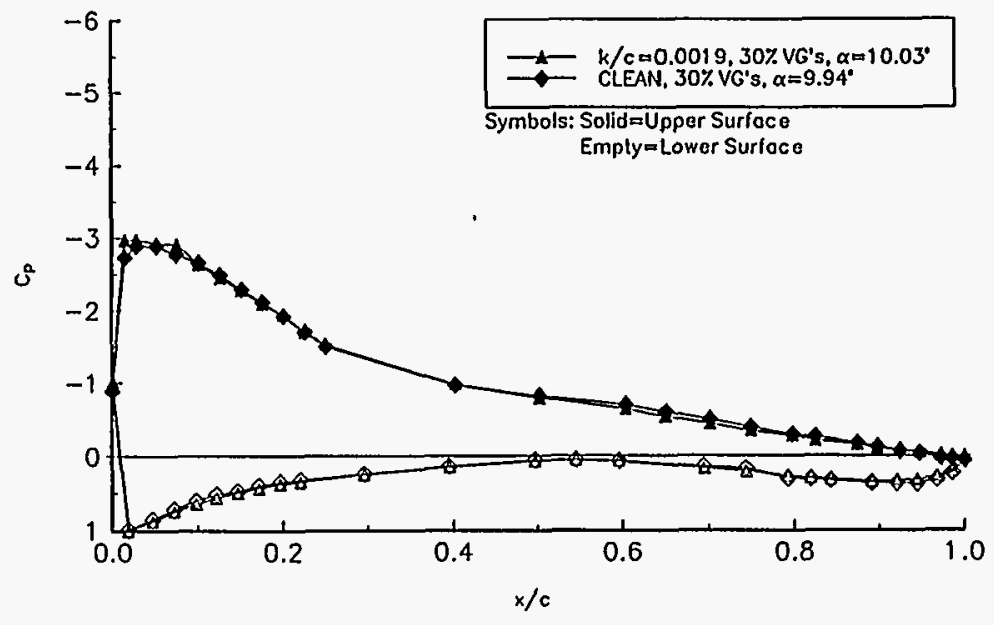

Figure 111. $\alpha=10^{\circ}$ 
$C_{p}$ VERSUS $x / c$

NASA LS(1)-0417MOD $\left(R_{0}=10 \times 10^{\circ}\right)$

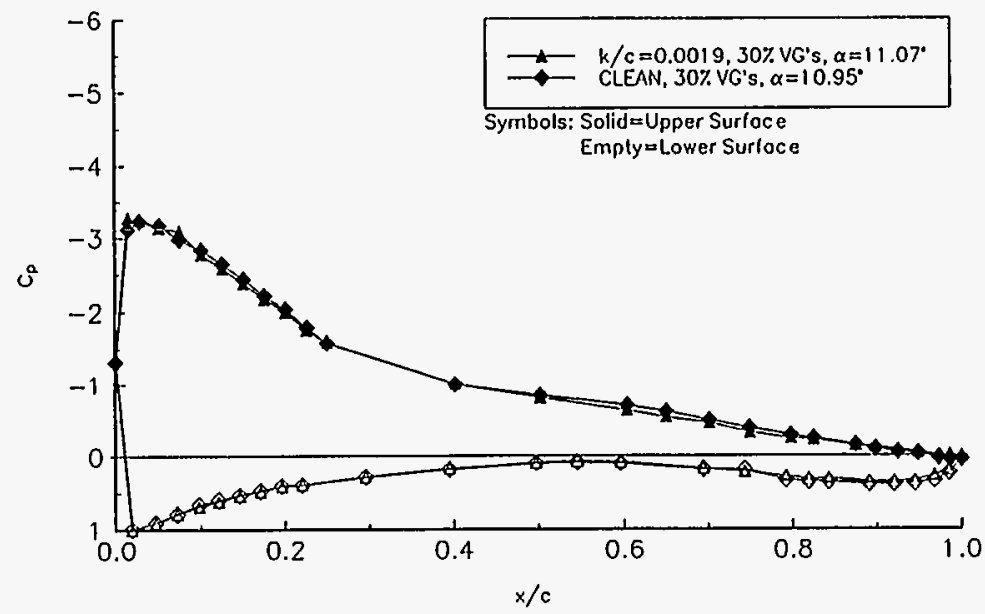

Figure 112. $\alpha=11^{\circ}$

$C_{p}$ VERSUS $x / c$

V

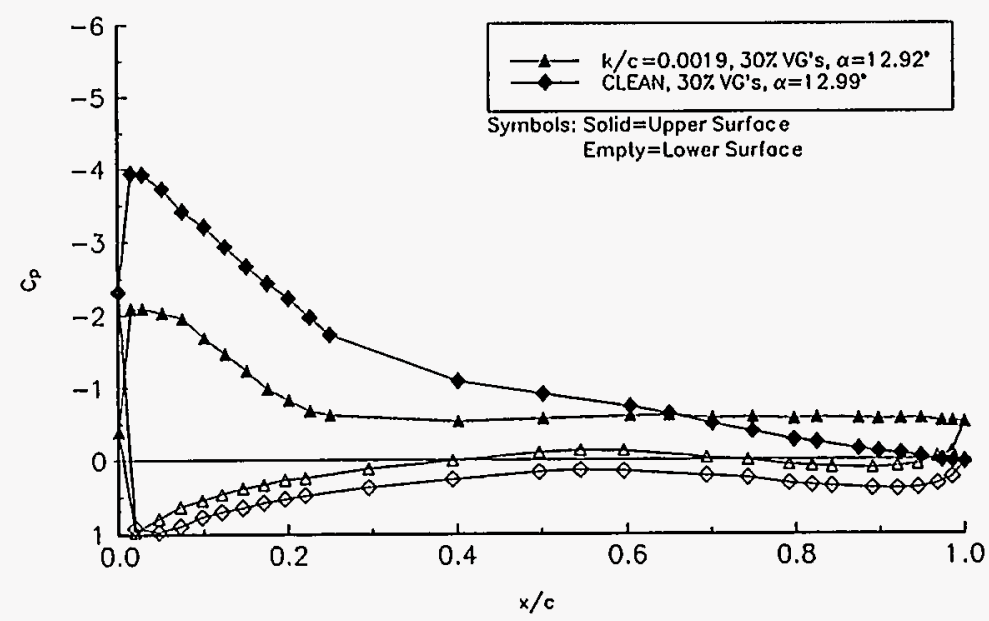

Figure 114. $\alpha=13^{\circ}$
NASA LS(1) -0417 MOD $\left(R_{0}=1.0 \times 10^{6}\right)$

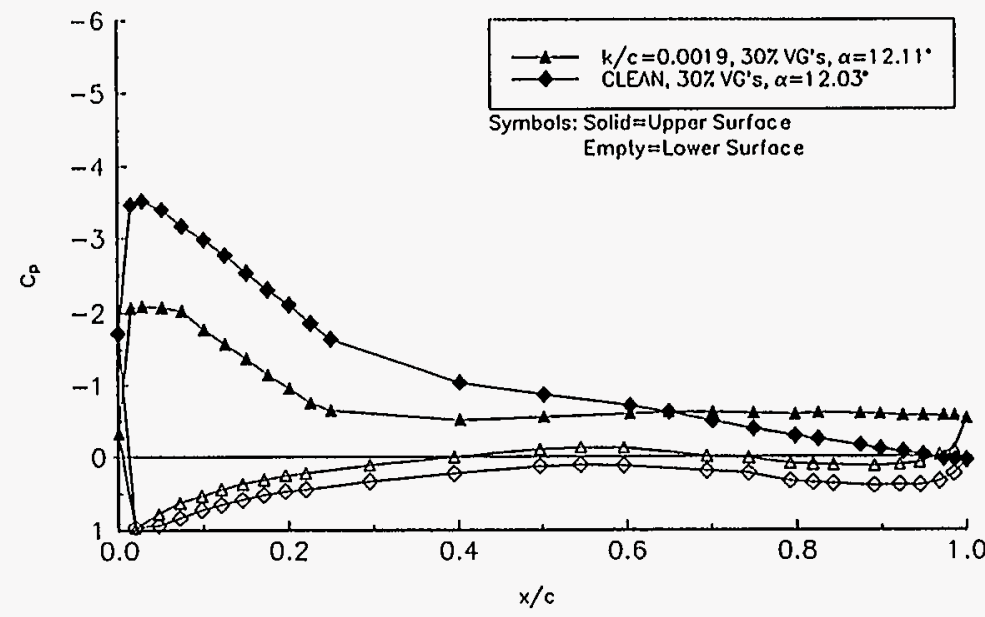

Figure 113. $\alpha=12^{\circ}$

VERSUS $x / c$

NASALS(1) $-0417 \mathrm{MOO}\left(R_{0}=1.0 \times 10^{6}\right)$

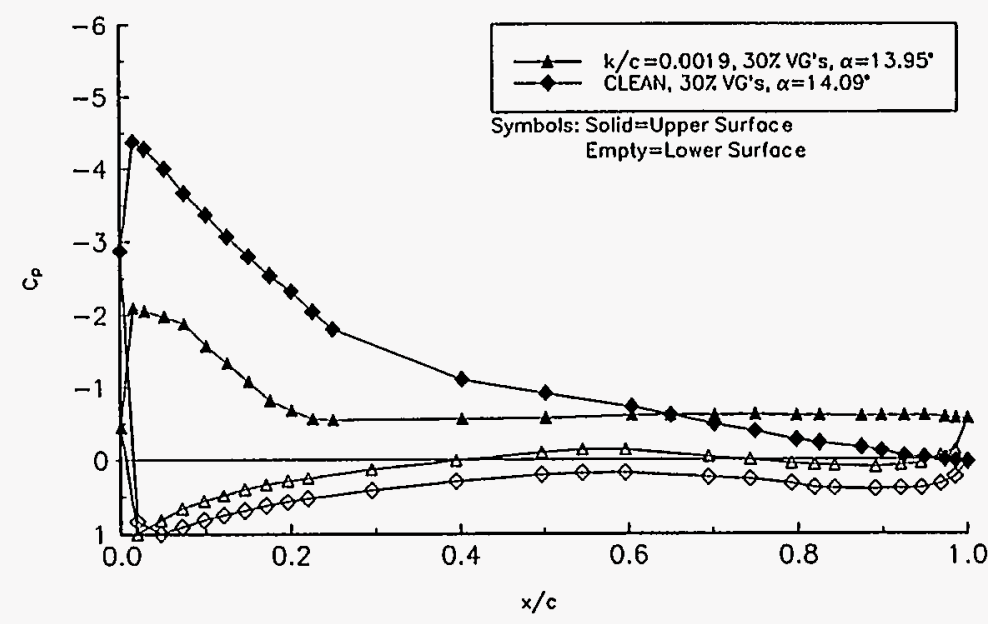

Figure 115. $\alpha=14^{\circ}$ 
$C_{p}$ VERSUS $x / c$

NASALS(1)-0417MOD $\left(R_{0}=1.0 \times 10^{6}\right)$

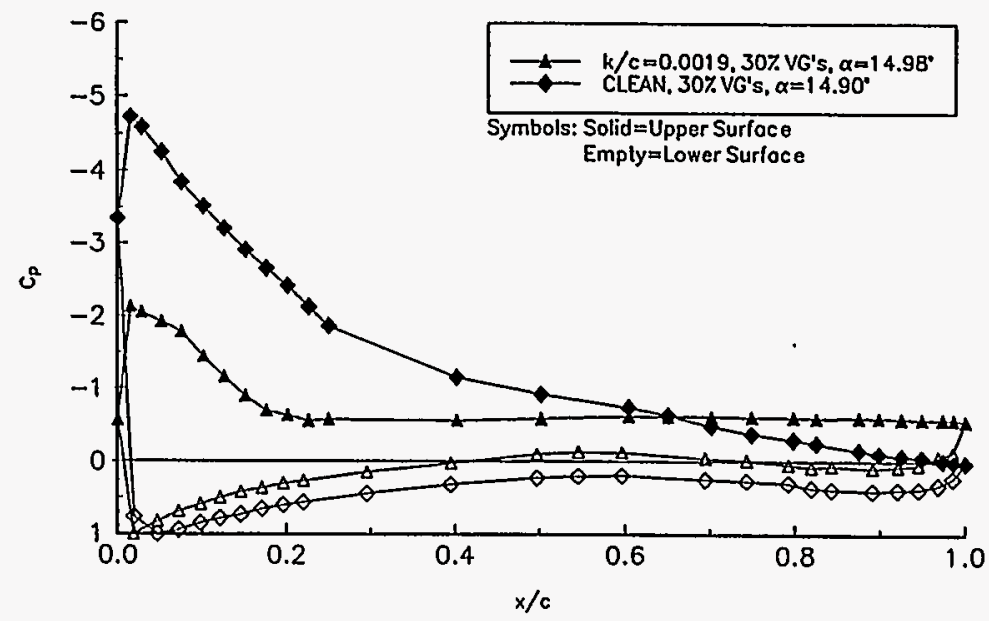

Figure 116. $\alpha=15^{\circ}$

$C_{p}$ VERSUS $x / c$

NASA LS(1)-0417 MOD $\left(R_{e}=1.0 \times 10^{6}\right)$

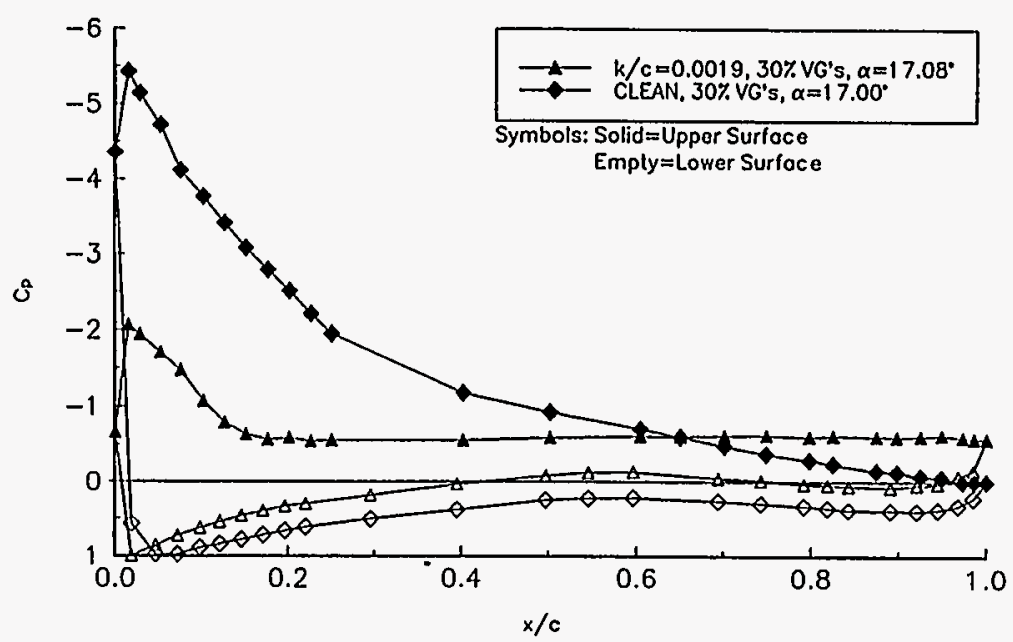

Figure 118. $\alpha=17^{\circ}$
$C_{p}$ VERSUS $x / c$

NASA LS(1) -0417 MOD $\left(R_{B}=1.0 \times 10^{\circ}\right)$

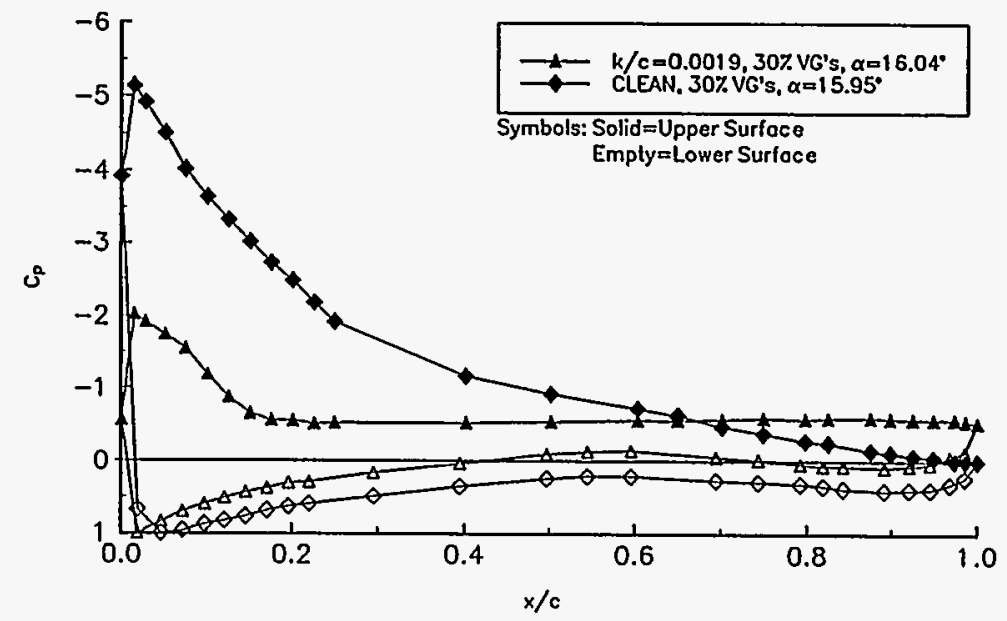

Figure 117. $\alpha=16^{\circ}$

$\mathrm{C}_{\mathrm{p}}$ VERSUS $\times / \mathrm{c}$

NASA LS $(1)-0417 \mathrm{MOD}\left(R_{\mathrm{g}}=1.0 \times 10^{6}\right)$

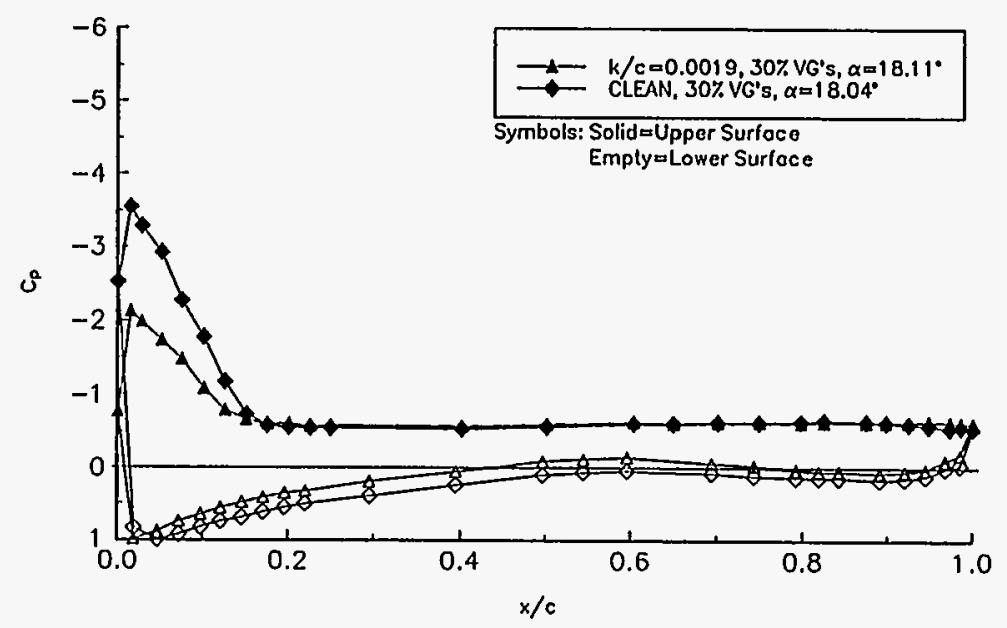

Figure 119. $\alpha=18^{\circ}$ 
$C_{\rho}$ VERSUS $x / c$

NASA LS(1)-0417 MOD $\left(R_{e}=1.0 \times 10^{6}\right)$

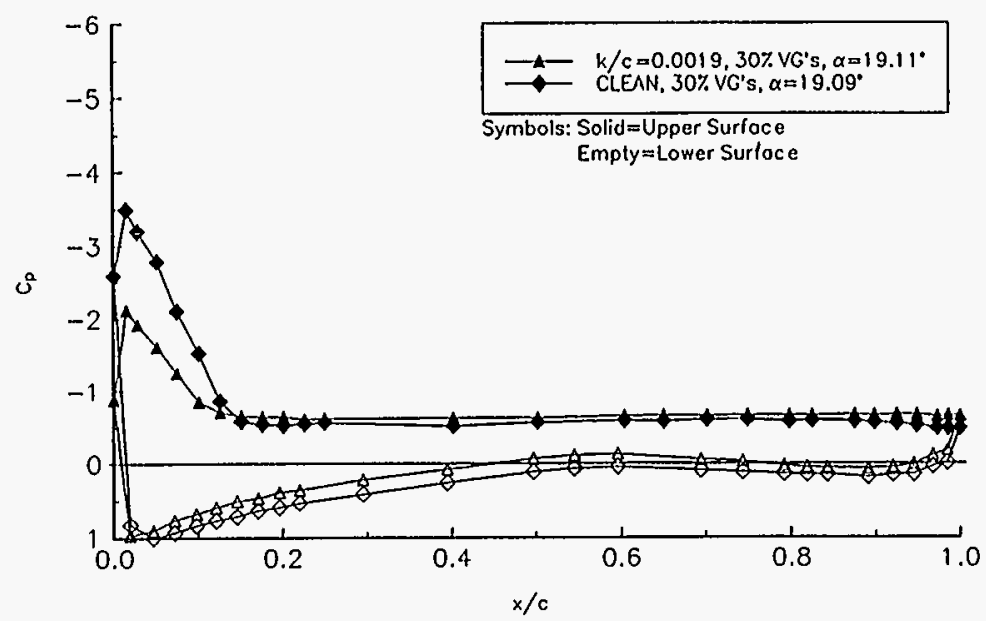

Figure 120. $\alpha=19^{\circ}$

$C_{P}$ VERSUS $x / c$

NASA LS(1)-0417 MOD $\left(R_{0}=1.0 \times 10^{6}\right)$

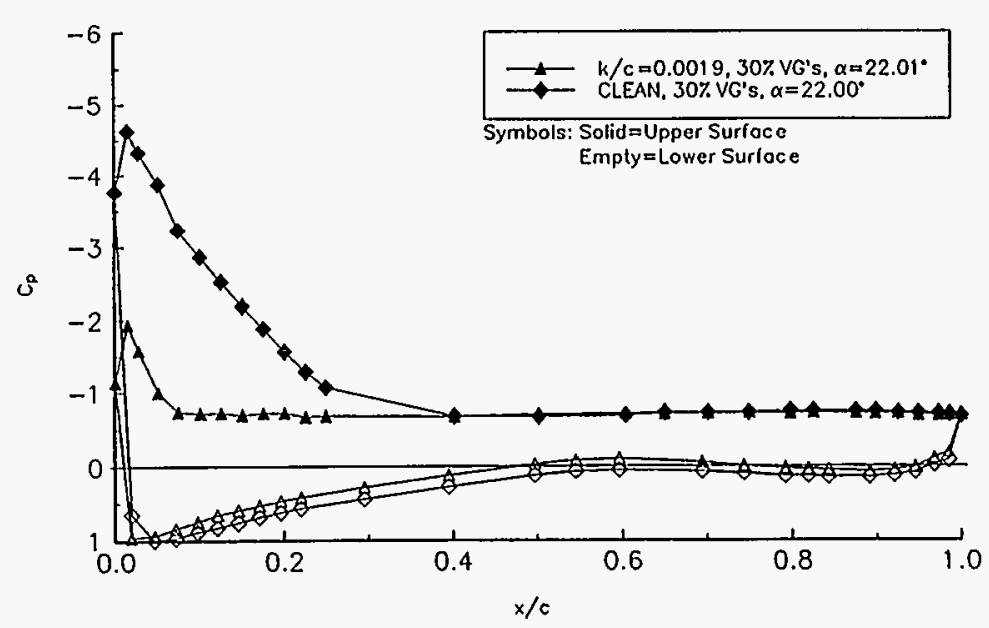

Figure 122. $\alpha=22^{\circ}$
NASA LS $(1)-0417$ MOD $\left(R_{8}=1.0 \times 10^{6}\right)$

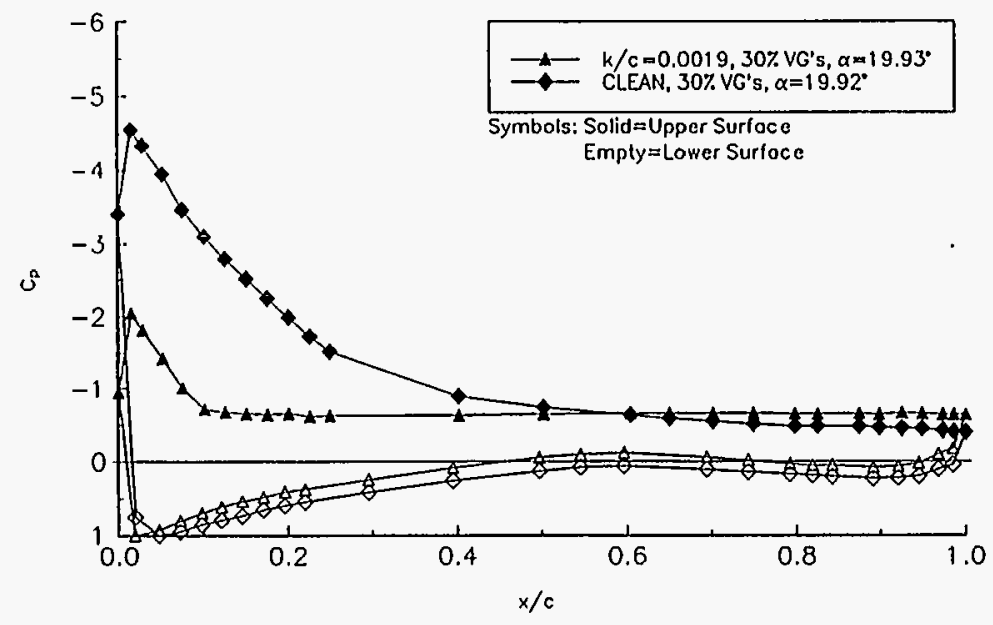

Figure 121. $\alpha=20^{\circ}$

$C_{p}$ VERSUS $x / c$

NASA LS $(1)-0417 \mathrm{MOD}\left(R_{0}=1.0 \times 10^{6}\right)$

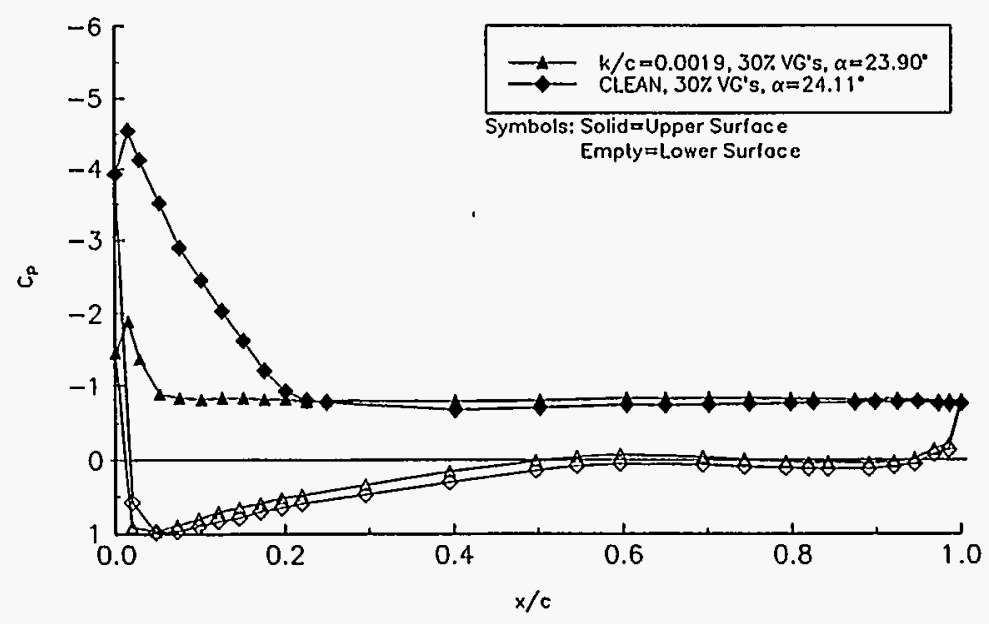

Figure 123. $\alpha=24^{\circ}$ 
Steady State Pressure Distributions

$\operatorname{Re}=1.5$ million 
$C_{p}$ VERSUS $x / c$

$\operatorname{LS}(1) 0417 \mathrm{MOD}\left(R_{0}=1.5 \times 10^{6}\right)$

i

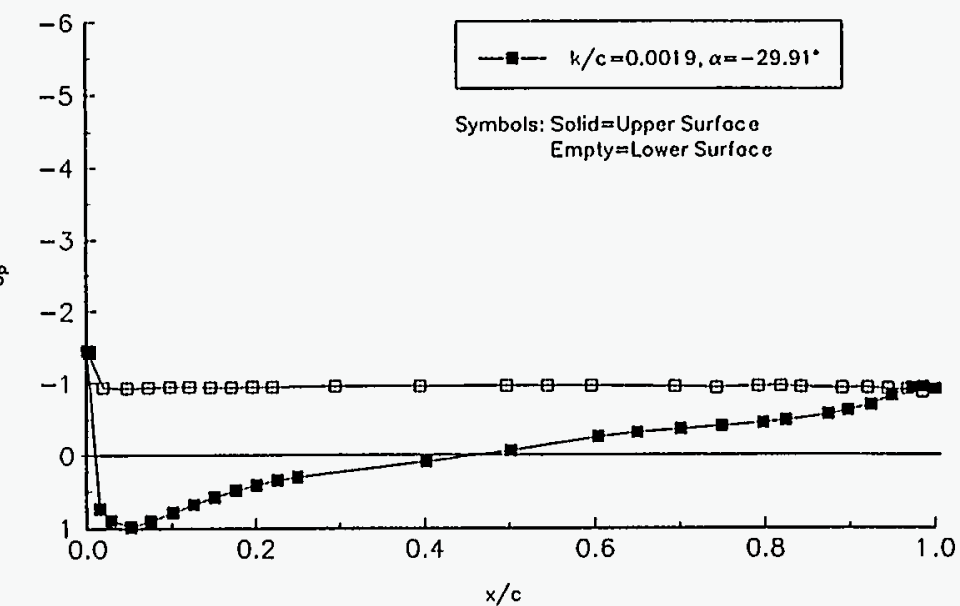

Figure 124. $\alpha=-30^{\circ}$

$C_{p}$ VERSUS $x / c$

LS(1) 0417 MOD $\left(R_{e}=1.5 \times 10^{6}\right)$

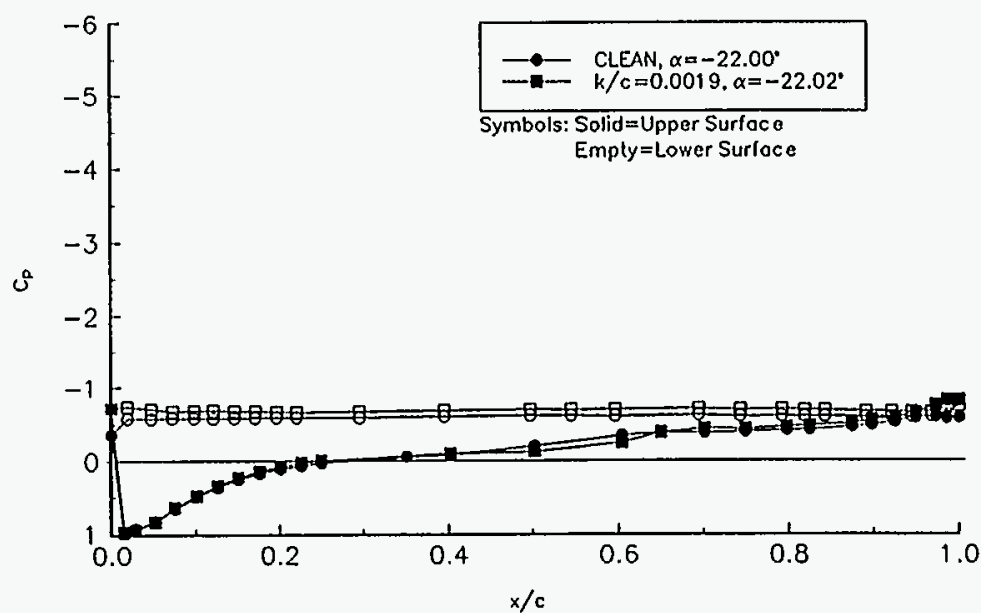

Figure 126. $\alpha=-22^{\circ}$
C VERSUS $x / c$

$\operatorname{LS}(1) 0417 \mathrm{MOD}\left(R_{e}=1.5 \times 10^{6}\right)$

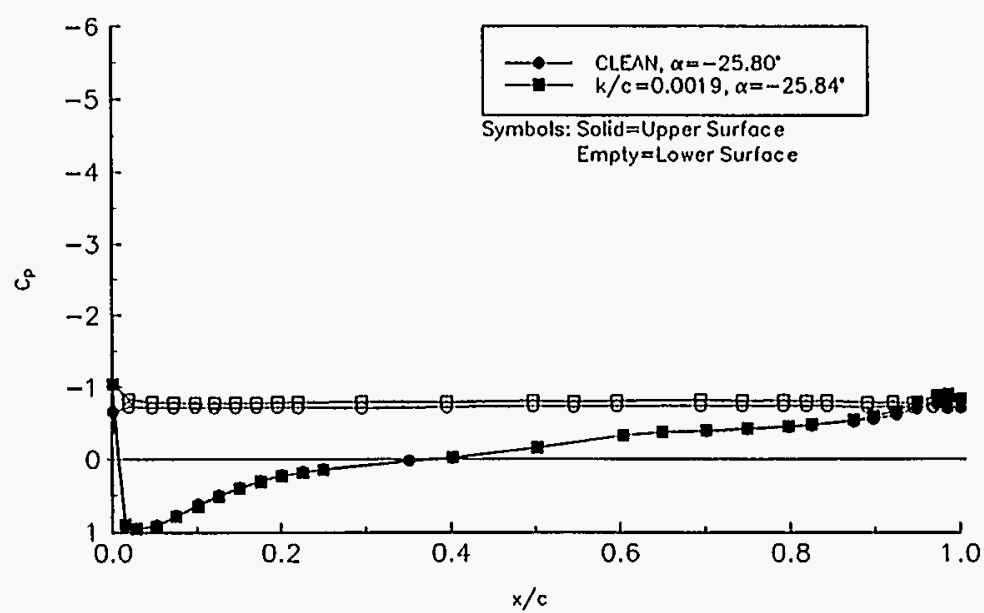

Figure 125. $\alpha=-26^{\circ}$

$C_{p}$ VERSUS $x / c$

LS(1) 0417 MOD $\left(R_{e}=1.5 \times 10^{6}\right)$

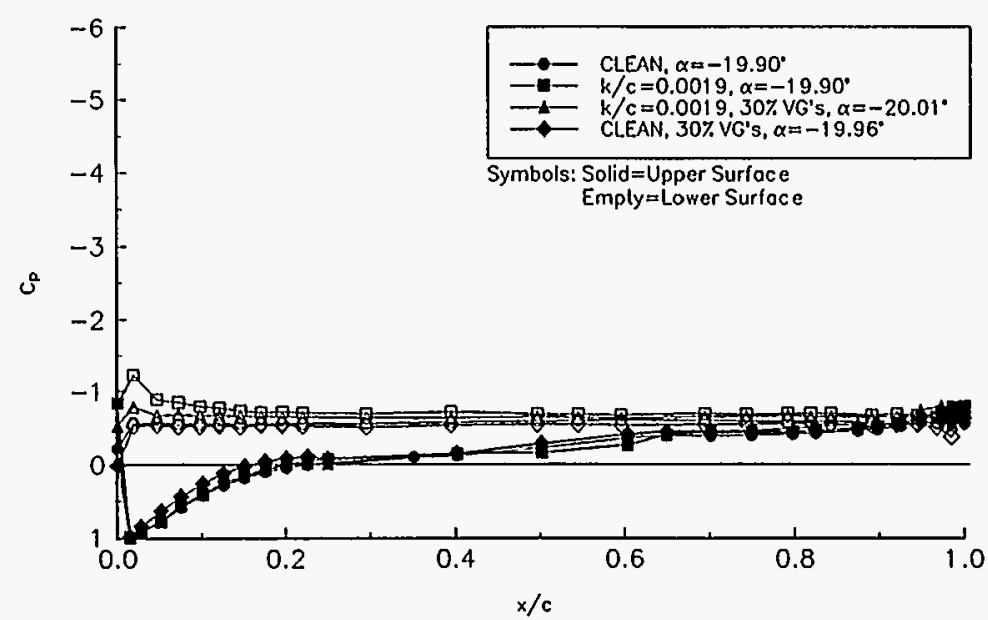

Figure 127. $\alpha=-20^{\circ}$ 
C $\mathrm{p}$ VERSUS $x / \mathrm{c}$

LS(1) $0417 \operatorname{MOD}\left(R_{e}=1.5 \times 10^{6}\right)$

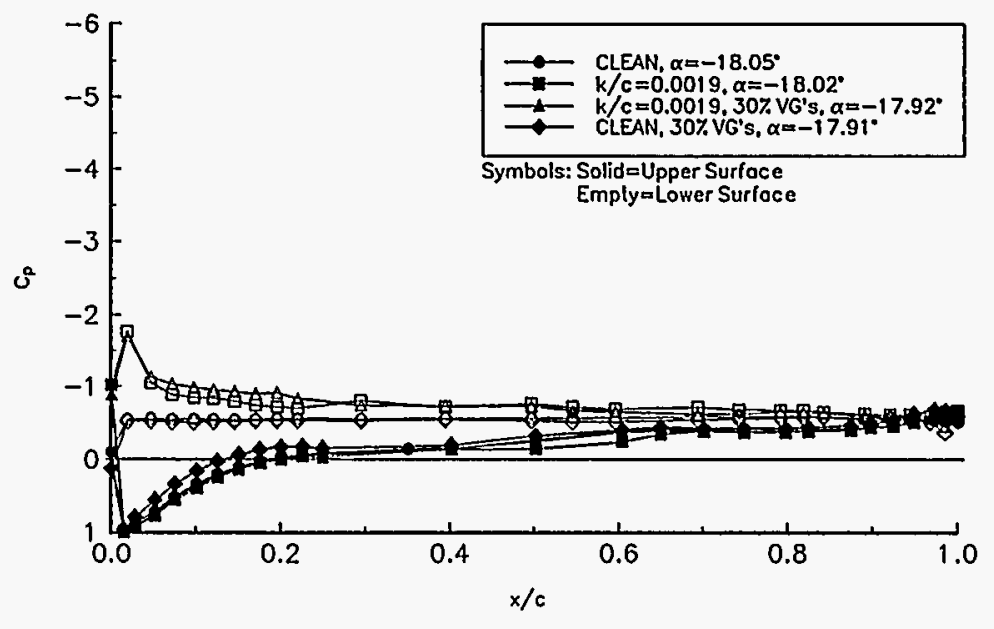

Figure 128. $\alpha=-18^{\circ}$

$C_{p}$ VERSUS $x / c$

$\operatorname{LS}(1) 0417 \operatorname{MOD}\left(R_{a}=1.5 \times 10^{6}\right)$

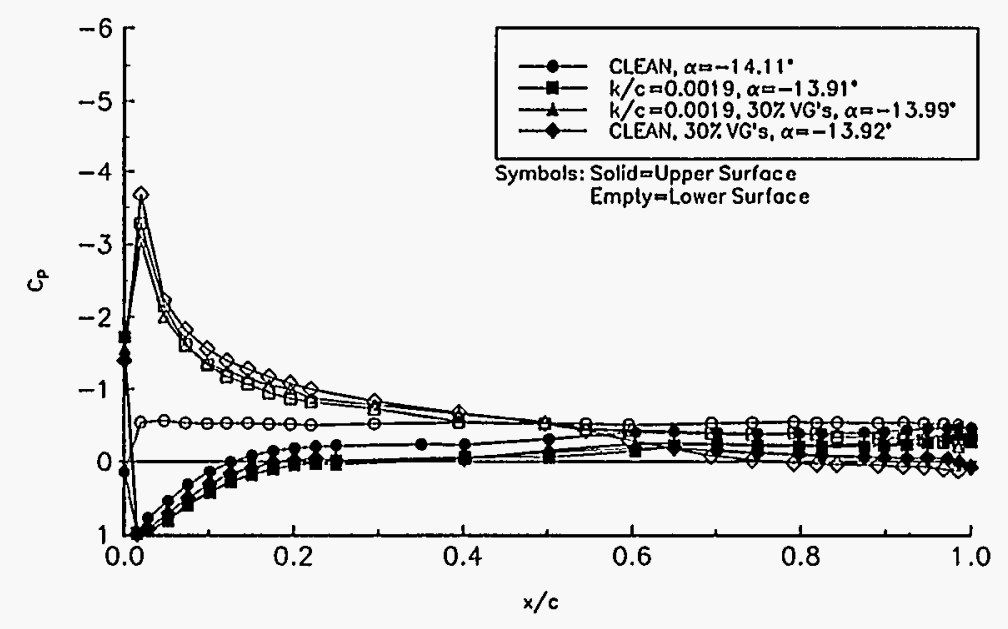

Figure 130. $\alpha=-14^{\circ}$
$C_{p}$ VERSUS $\times / c$
$\operatorname{LS}(1) 0417 \operatorname{MOD}\left(R_{\mathrm{e}}=1.5 \times 10^{6}\right)$

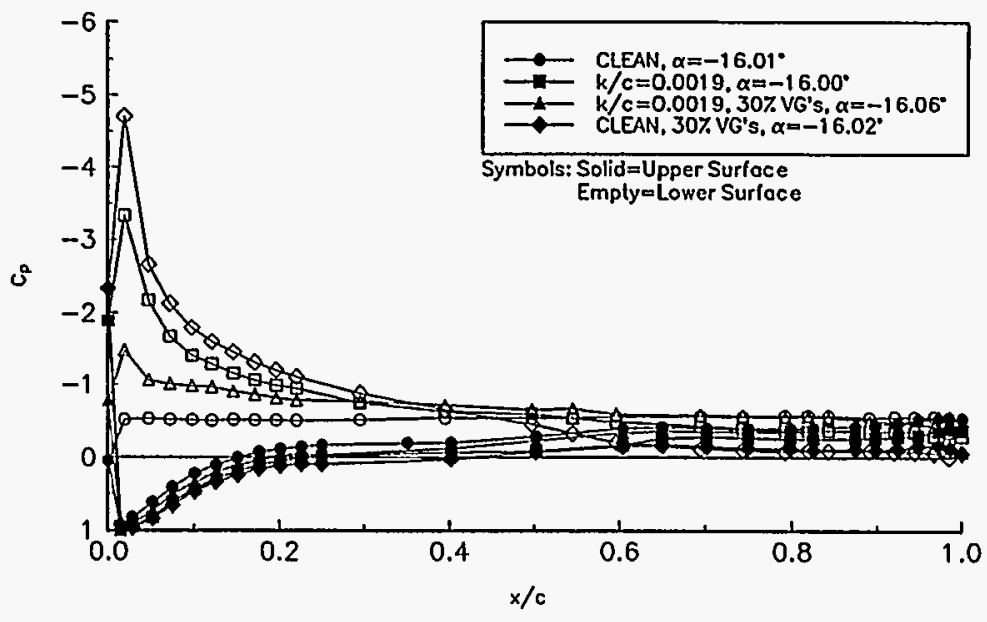

Figure 129. $\alpha=-16^{\circ}$

$C_{p}$ VERSUS $x / c$ $\operatorname{LS}(1) 0417 \mathrm{MOD}\left(R_{\mathrm{B}}=1.5 \times 10^{6}\right)$

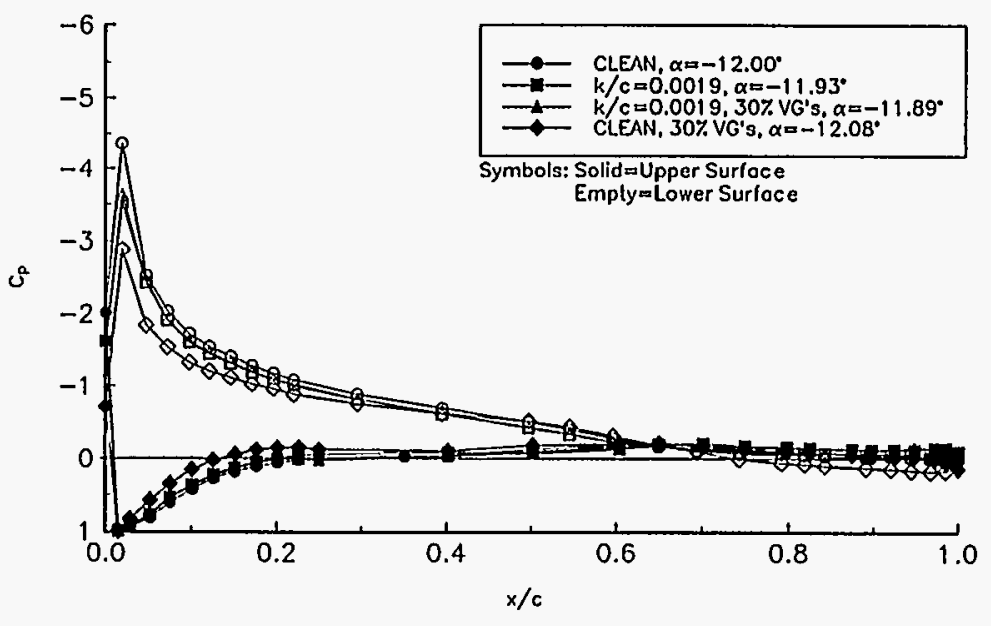

Figure 131. $\alpha=-12^{\circ}$ 
$C_{p}$ VERSUS $\times / c$
(1) 0417 MOD $\left(R_{\mathrm{e}}=1.5 \times 10^{6}\right)$

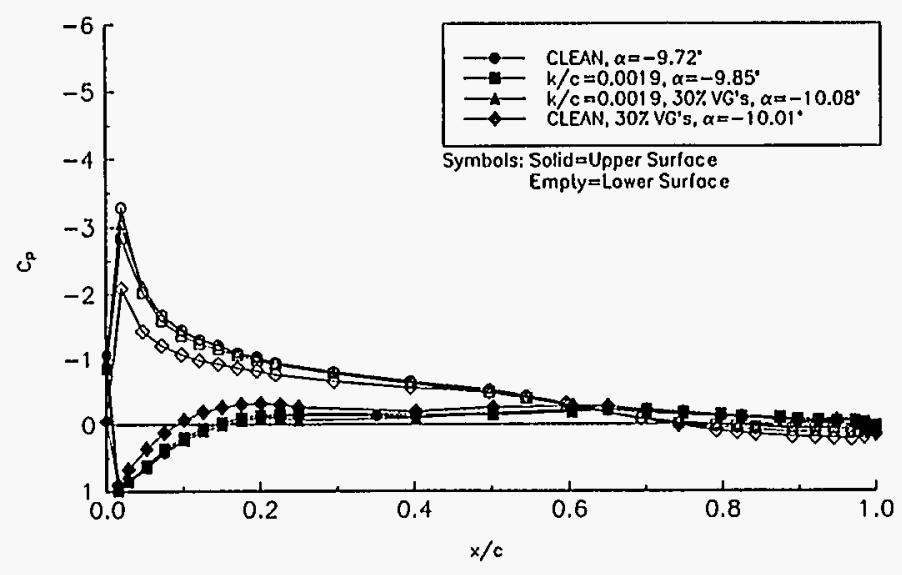

Figure 132. $\alpha=-10^{\circ}$

C. VERSUS $x / c$

$\operatorname{LS}(1) 0417 \mathrm{MOD}\left(R_{0}=1.5 \times 10^{6}\right)$

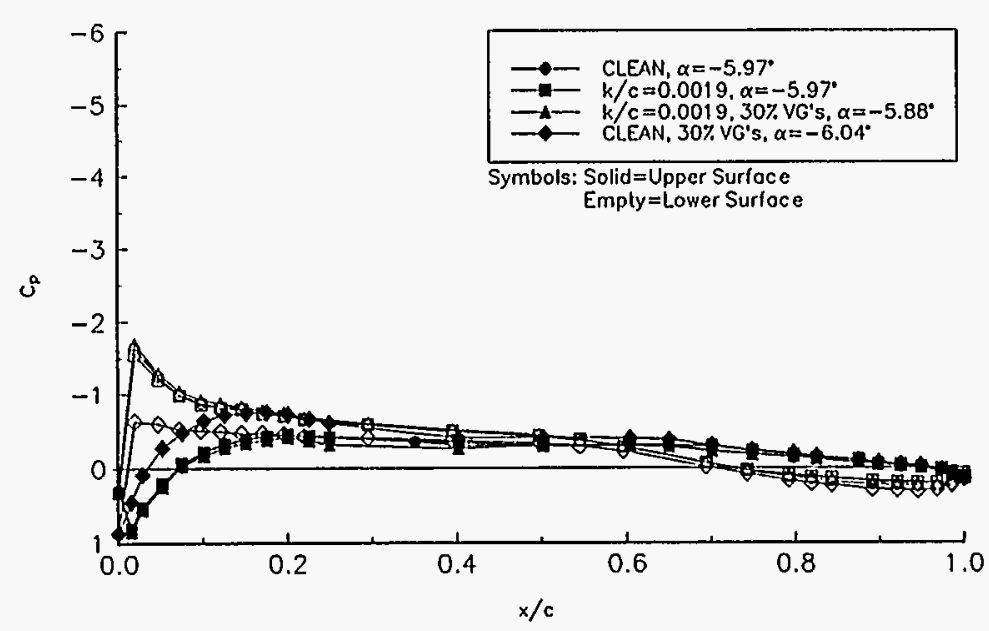

Figure 134. $\alpha=-6^{\circ}$
$C_{\rho}$ VERSUS $x / c$

$\operatorname{LS}(1) 0417 \mathrm{MOD}\left(R_{e}=1.5 \times 10^{6}\right)$

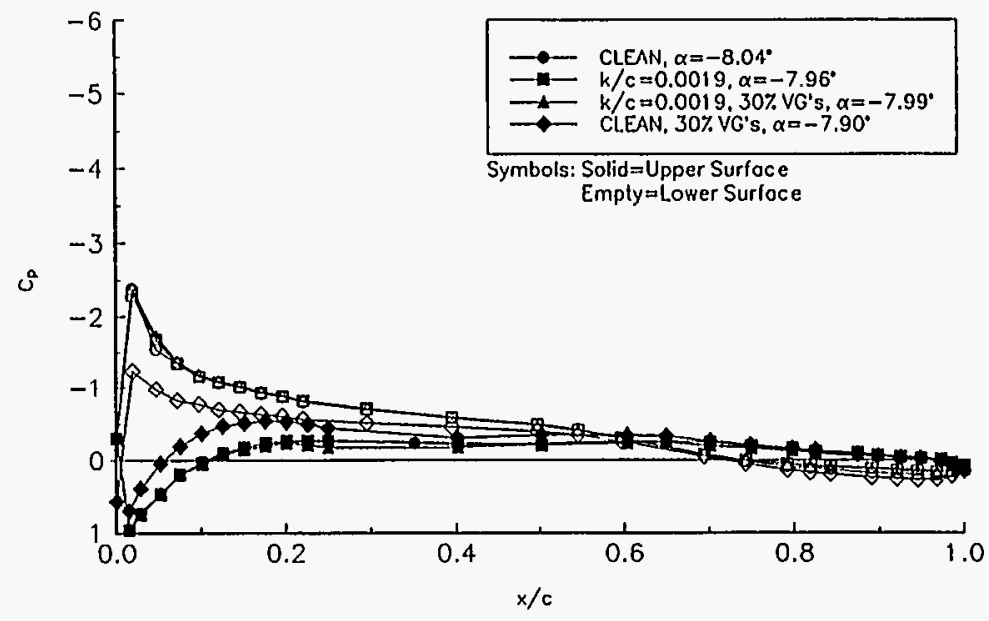

Figure 133. $\alpha=-8^{\circ}$

$C_{p}$ VERSUS $x / c$

LS(1) $0417 \mathrm{MOD}\left(R_{\mathrm{e}}=1.5 \times 10^{6}\right)$

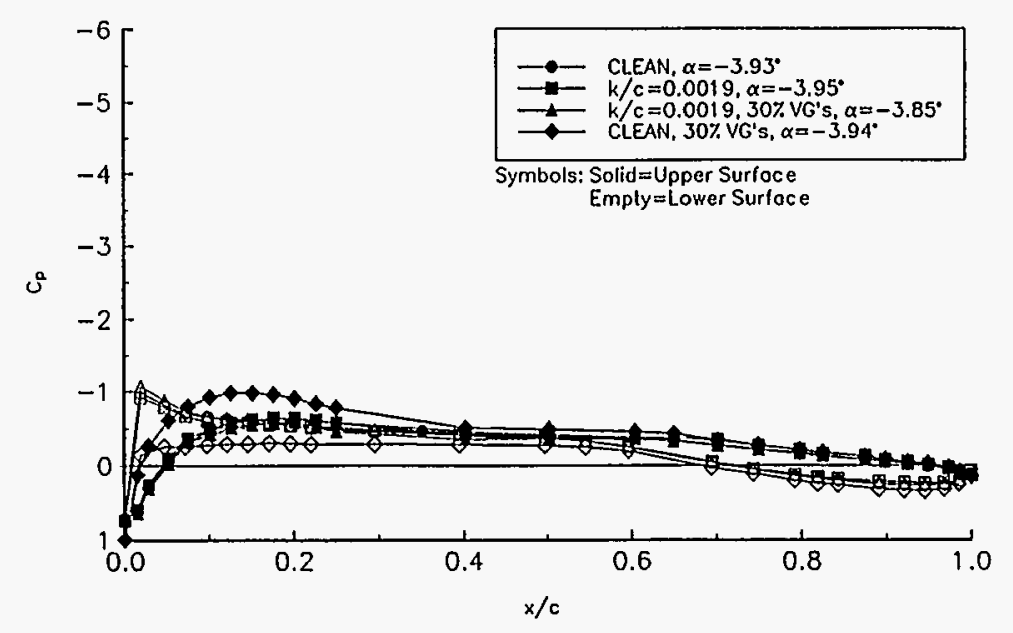

Figure 135. $\alpha=-4^{\circ}$ 
$C_{p}$ VERSUS $x / c$

LS(1) $0417 \mathrm{MOD}\left(R_{\theta}=1.5 \times 10^{6}\right)$

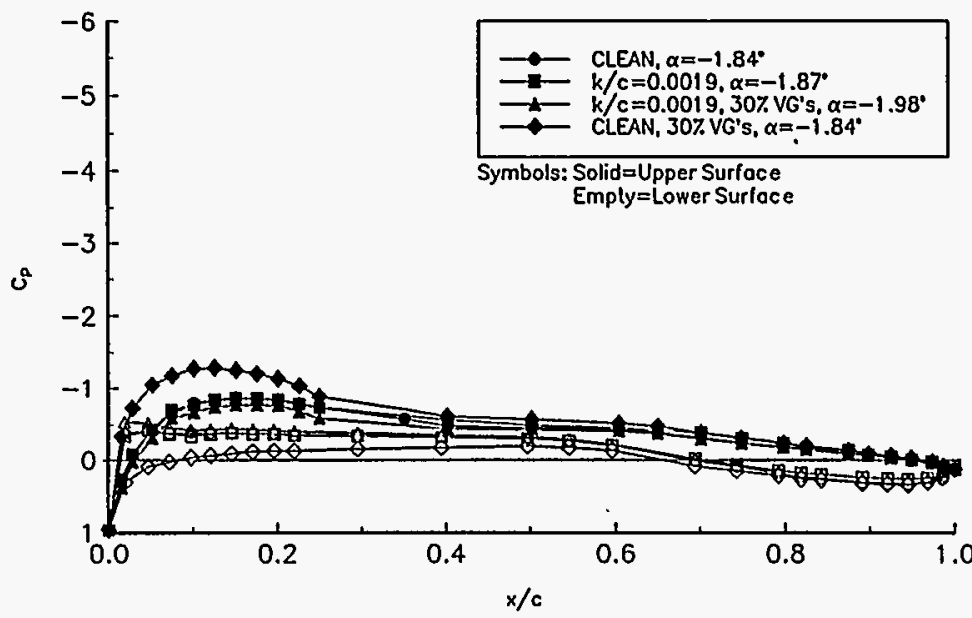

Figure 136. $\alpha=-2^{\circ}$

$C_{p}$ VERSUS $x / c$

$\operatorname{LS}(1) 0417 \mathrm{MOD}\left(R_{\theta}=1.5 \times 10^{\circ}\right)$

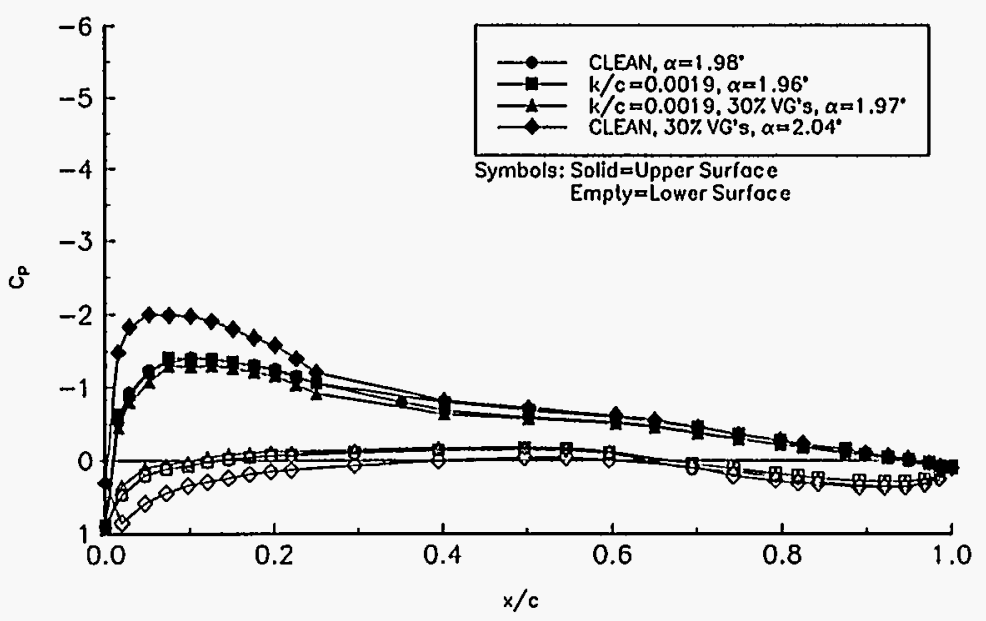

Figure 138. $\alpha=2^{\circ}$
$C_{p}$ VERSUS $x / \mathrm{c}$

$L S(1) 0417 \mathrm{MOD}\left(R_{0}=1.5 \times 10^{6}\right)$

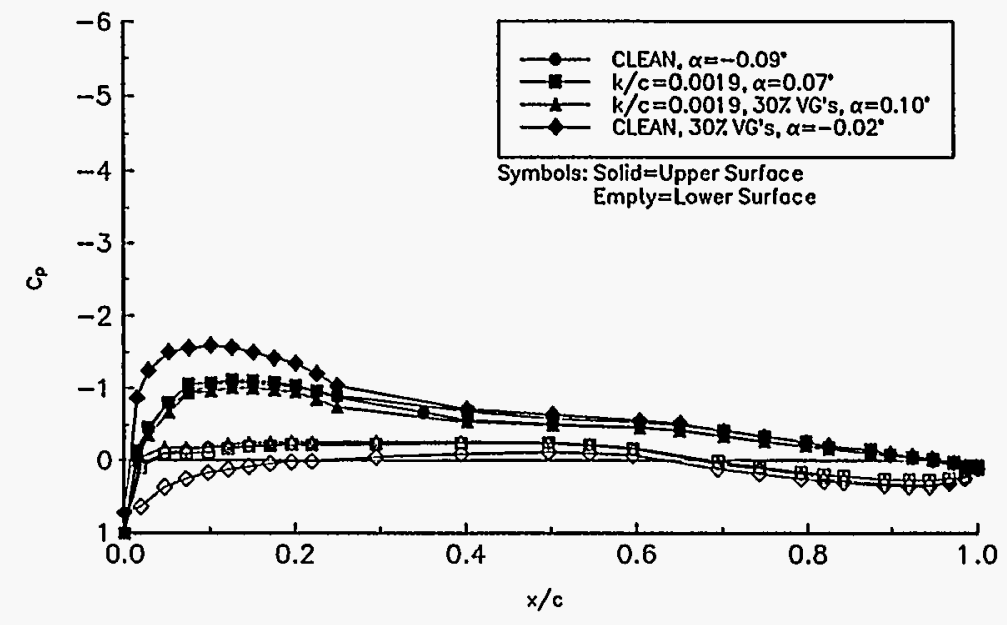

Figure 137. $\alpha=0^{\circ}$

$C_{p}$ VERSUS $x / c$ $\operatorname{LS}(1) 0417 \mathrm{MOD}\left(R_{\mathrm{a}}=1.5 \times 10^{6}\right)$

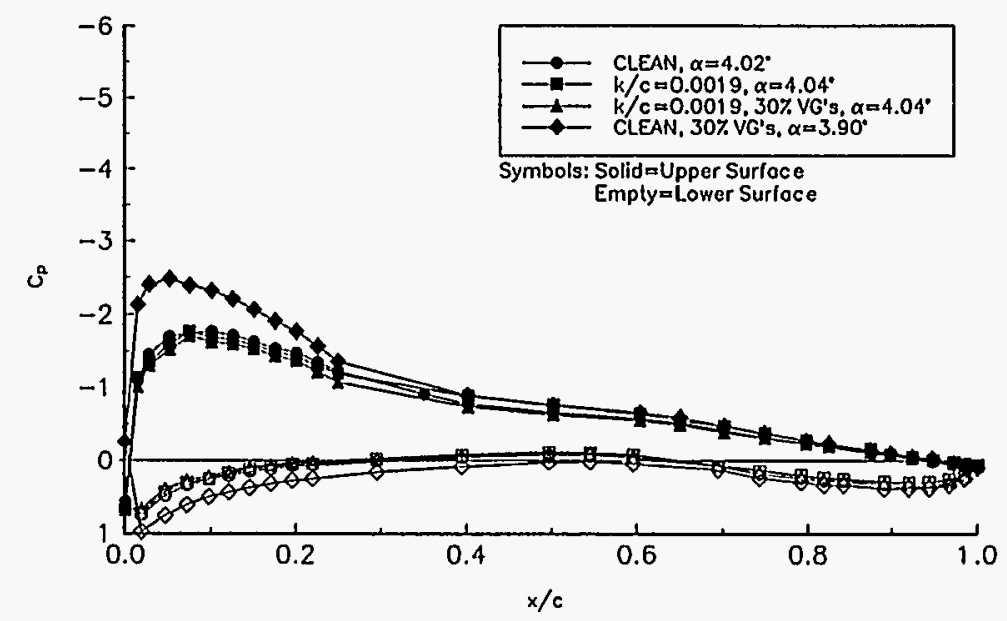

Figure 139. $\alpha=4^{\circ}$ 
C. VERSUS $x / c$

$\operatorname{LS}(1) 0417 \mathrm{MOD}\left(R_{0}=1.5 \times 10^{8}\right)$

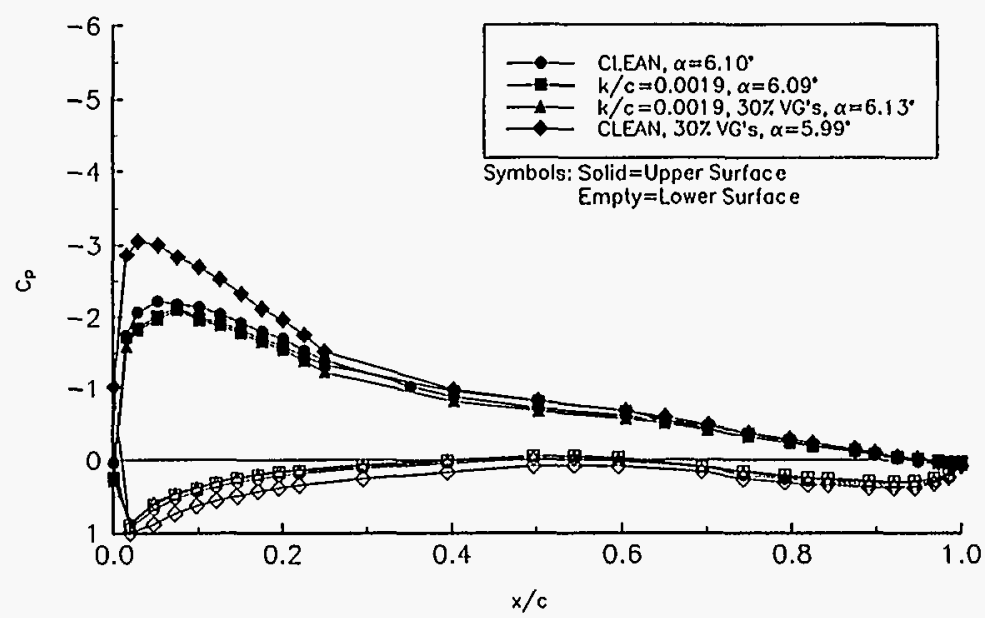

Figure 140. $\alpha=6^{\circ}$

$C_{p}$ VERSUS $x / c$

$\operatorname{LS}(1) 0417 \mathrm{MOD}\left(R_{e}=1.5 \times 10^{6}\right)$

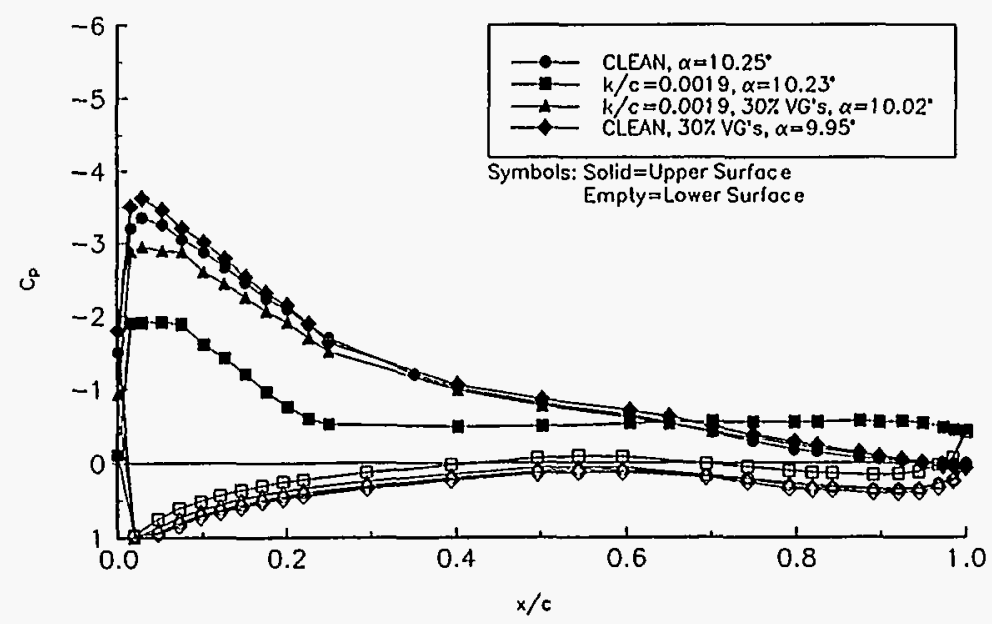

Figure 142. $\alpha=10^{\circ}$
$\mathrm{C}_{p}$ VERSUS $x / \mathrm{c}$

$\operatorname{LS}(1) 0417 \mathrm{MOD}\left(R_{\mathrm{e}}=1.5 \times 10^{\circ}\right)$

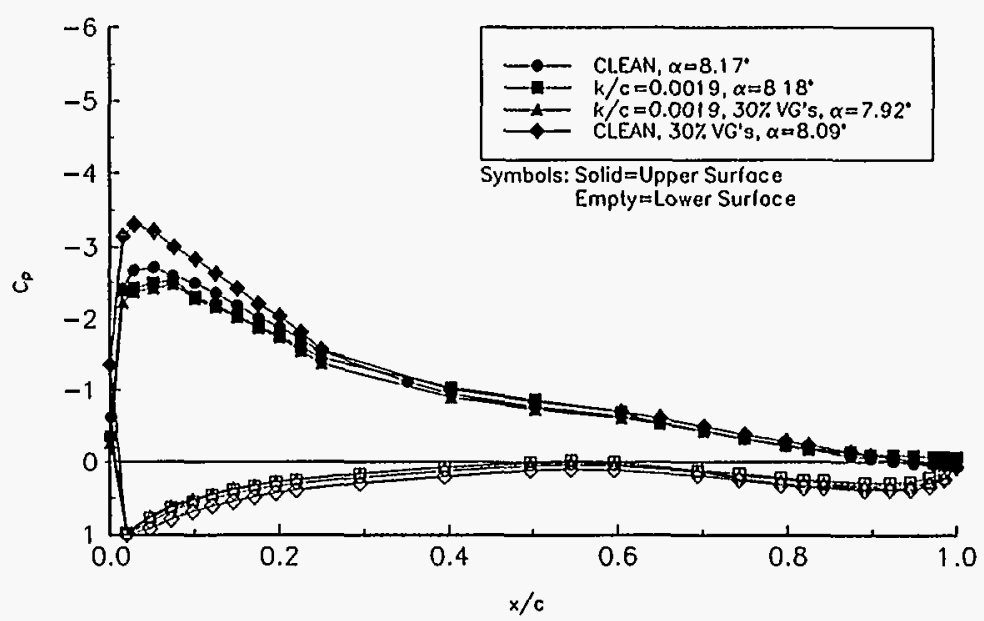

Figure 141. $\alpha=8^{\circ}$

$C_{\rho}$ VERSUS $x / c$

$\operatorname{LS}(1) 0417 \mathrm{MOD}\left(R_{e}=1.5 \times 10^{6}\right)$

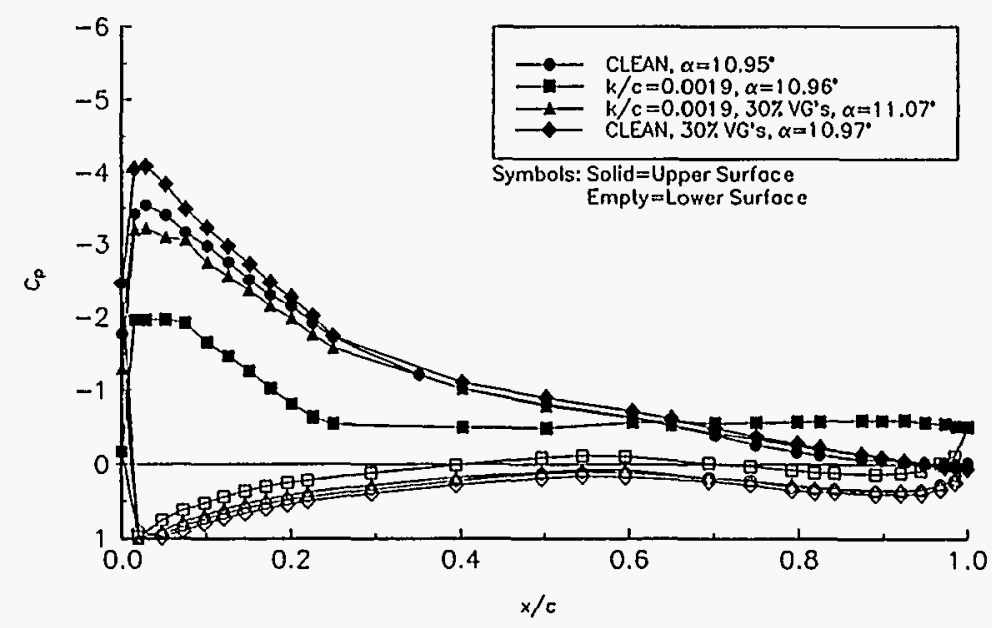

Figure 143. $\alpha=11^{\circ}$ 
$\mathrm{C}_{\mathrm{p}}$ VERSUS $\mathrm{x} / \mathrm{c}$

$L S(1) 0417 \mathrm{MOD}\left(R_{\mathrm{e}}=1.5 \times 10^{6}\right)$

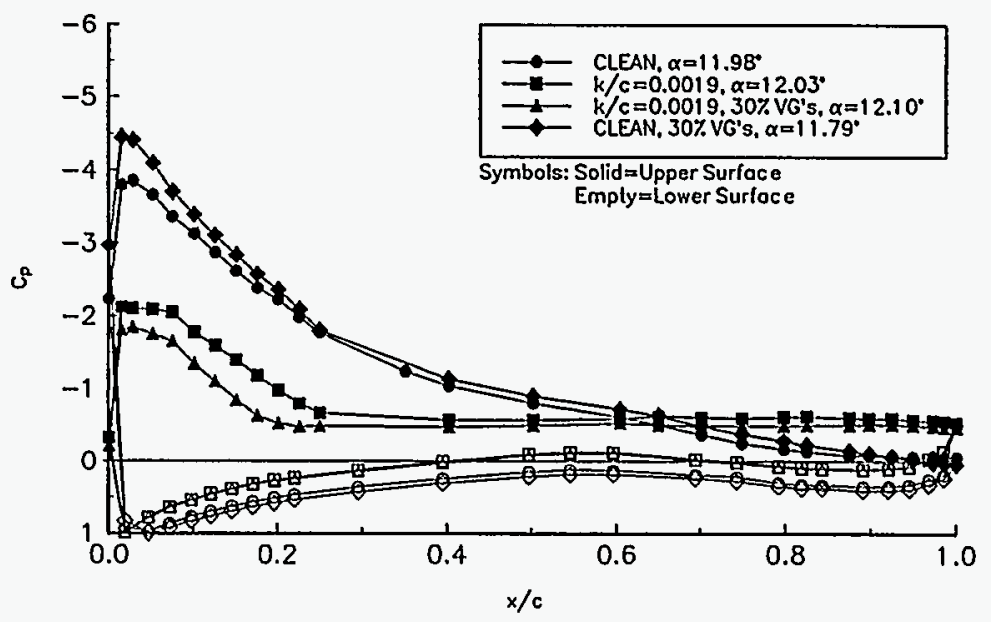

Figure 144. $\alpha=12^{\circ}$

VERSUS $x / \mathrm{c}$

$L S(1) 0417 \mathrm{MOD}\left(R_{0}=1.5 \times 10^{6}\right)$

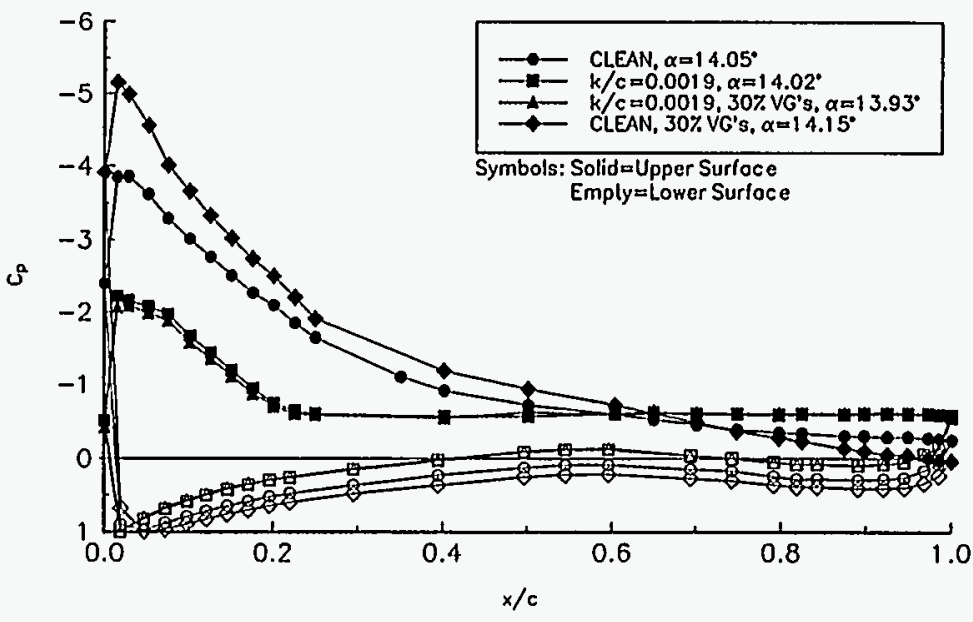

Figure 146. $\alpha=14^{\circ}$
$C_{p}$ VERSUS $x / c$

$L S(1) 0417 \mathrm{MOO}\left(R_{0}=1.5 \times 10^{6}\right)$

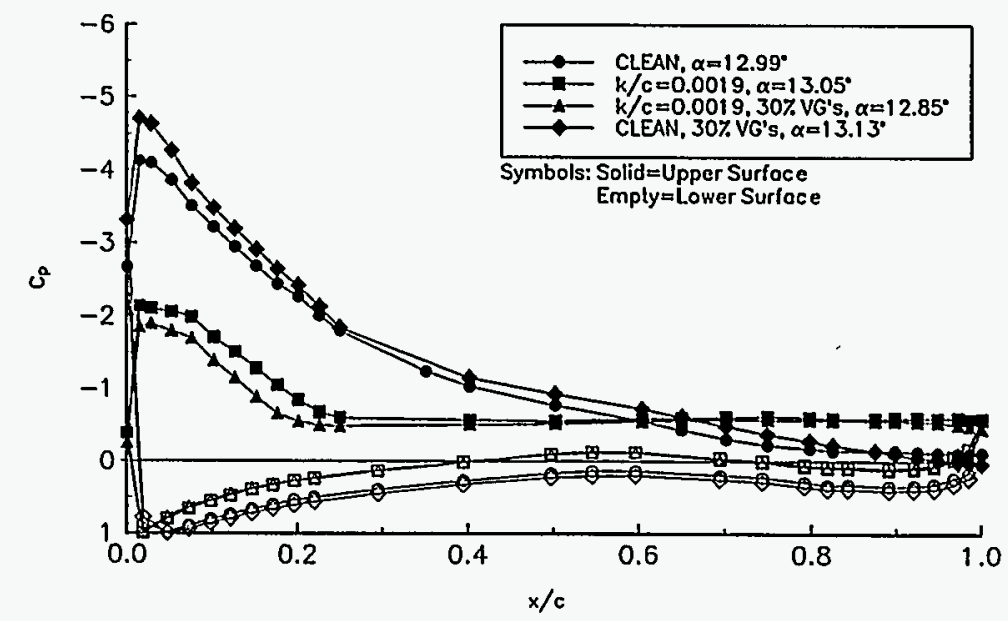

Figure 145. $\alpha=13^{\circ}$

LS(1) 0417 MOD $\left(R_{\theta}=1.5 \times 10^{8}\right)$

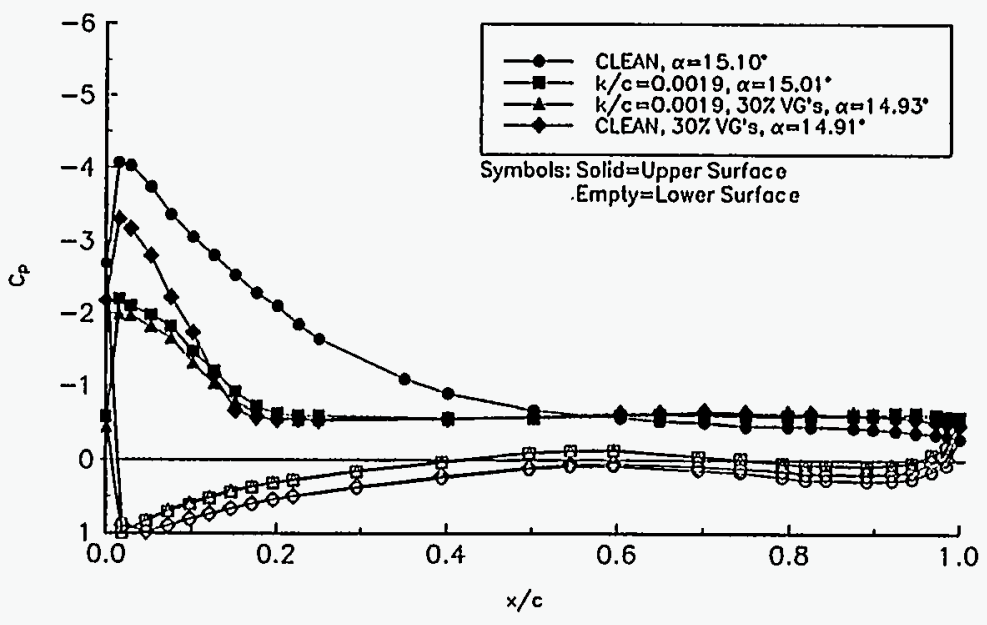

Figure 147. $\alpha=15^{\circ}$ 
$C_{0}$ VERSUS $x / C$

$\operatorname{LS}(1) 0417 \mathrm{MOD}\left(R_{\mathbf{e}}=1.5 \times 10^{\circ}\right)$

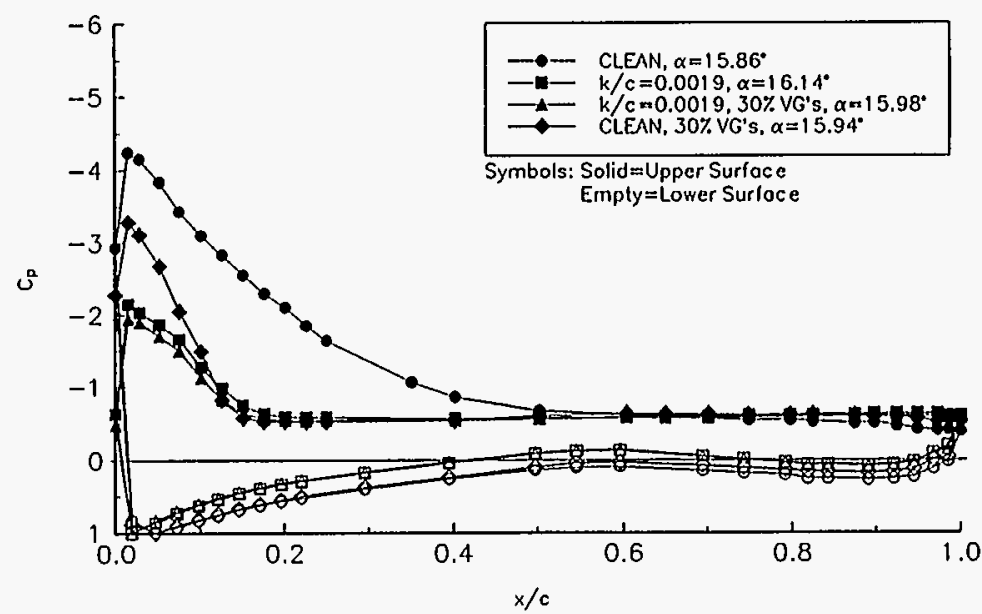

Figure 148. $\alpha=16^{\circ}$

$C_{p}$ VERSUS $x / c$

LS(1) 0417 MOD $\left(R_{\mathrm{e}}=1.5 \times 10^{6}\right)$

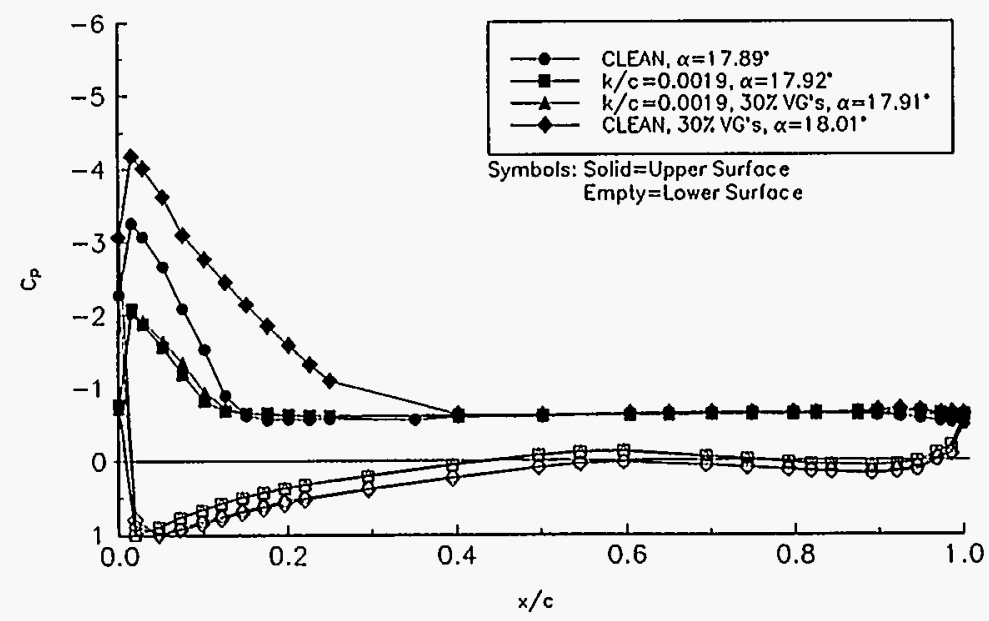

Figure 150. $\alpha=18^{\circ}$
$C_{p}$ VERSUS $x / c$

$\operatorname{LS}(1) 0417 \mathrm{MOD}\left(R_{e}=1.5 \times 10^{\circ}\right)$

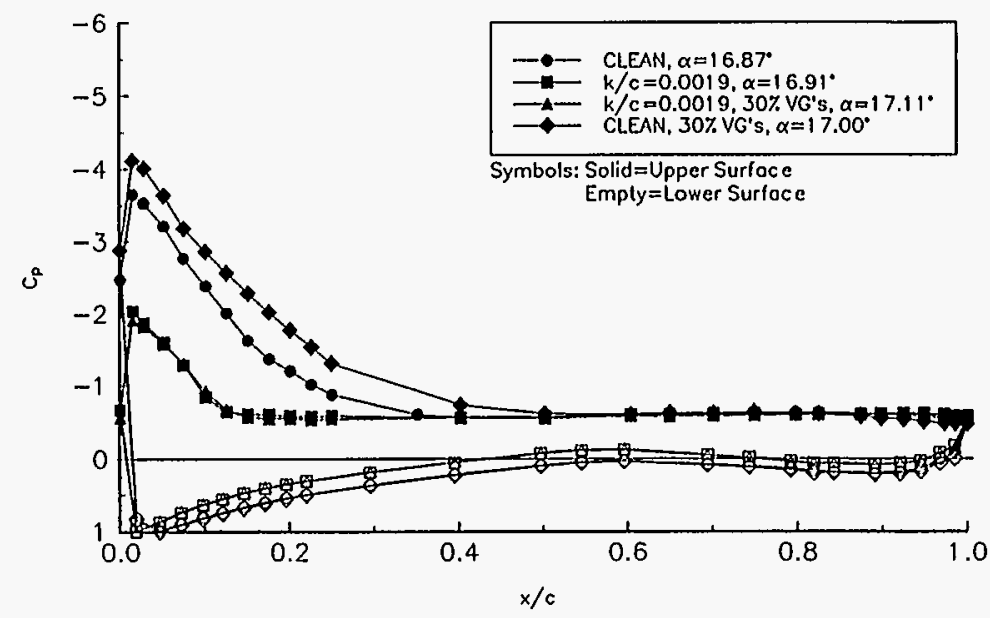

Figure 149. $\alpha=17^{\circ}$

$C_{P}$ VERSUS $x / c$

LS(1) $0417 \mathrm{MOD}\left(R_{e}=1.5 \times 10^{6}\right)$

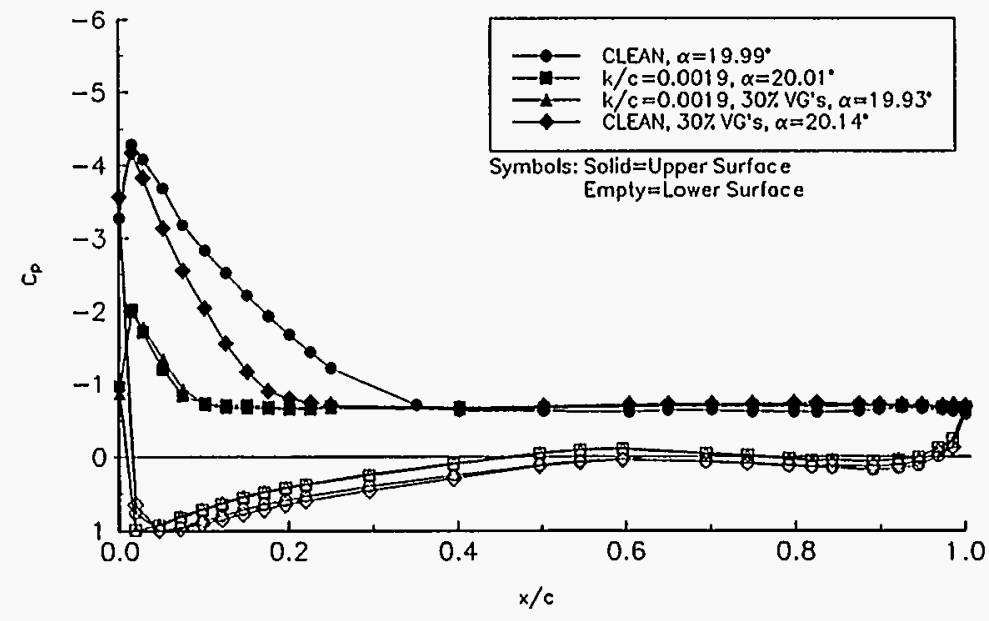

Figure 151. $\alpha=20^{\circ}$ 
$C_{p}$ VERSUS $x / c$

LS(1) $0417 \mathrm{MOD}\left(R_{\mathrm{e}}=1.5 \times 10^{6}\right)$

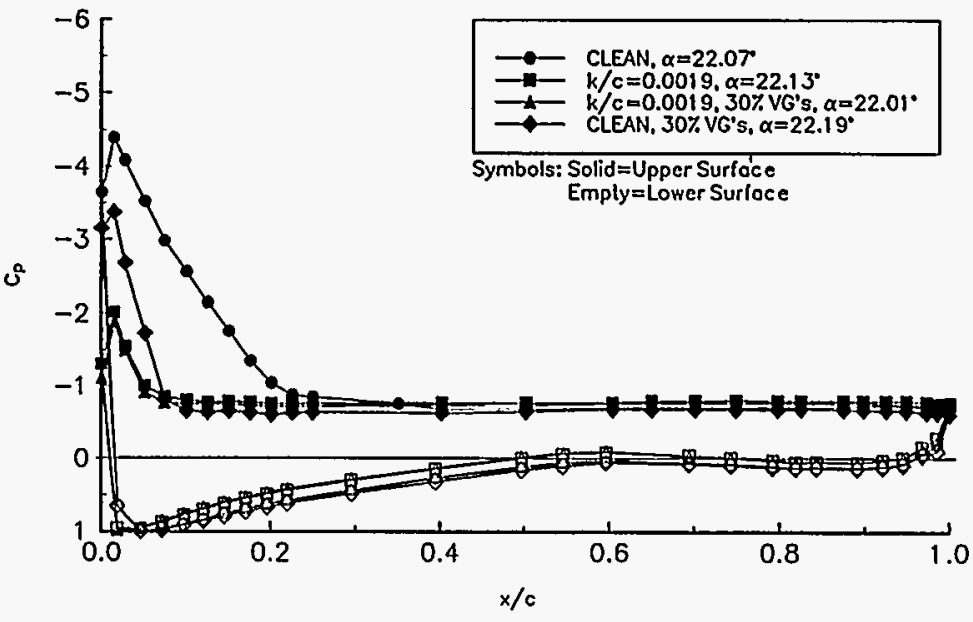

Figure 152. $\alpha=22^{\circ}$

$C_{p}$ VERSUS $x / c$

$\operatorname{LS}(1) 0417 \mathrm{MOD}\left(R_{\mathrm{g}}=1.5 \times 10^{\circ}\right)$

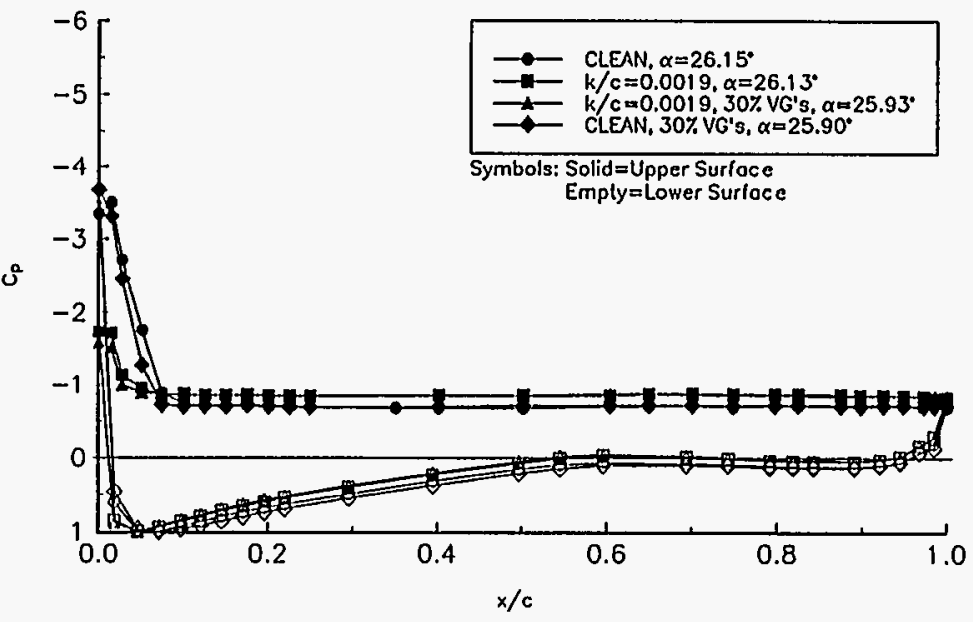

Figure 154. $\alpha=26^{\circ}$
$C_{p}$ VERSUS $x / c$

$\operatorname{LS}(1) 0417 \mathrm{MOD}\left(R_{\mathrm{e}}=1.5 \times 10^{6}\right)$

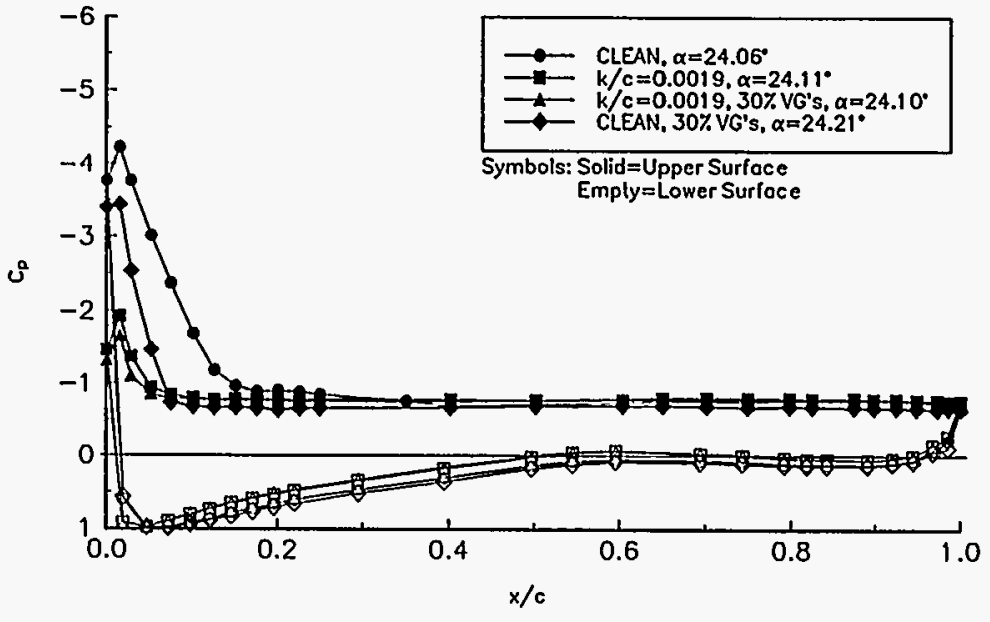

Figure 153. $\alpha=24^{\circ}$

$C_{p}$ VERSUS $x / c$

$\operatorname{LS}(1) 0417 \mathrm{MOD}\left(R_{\mathrm{e}}=1.5 \times 10^{\circ}\right)$

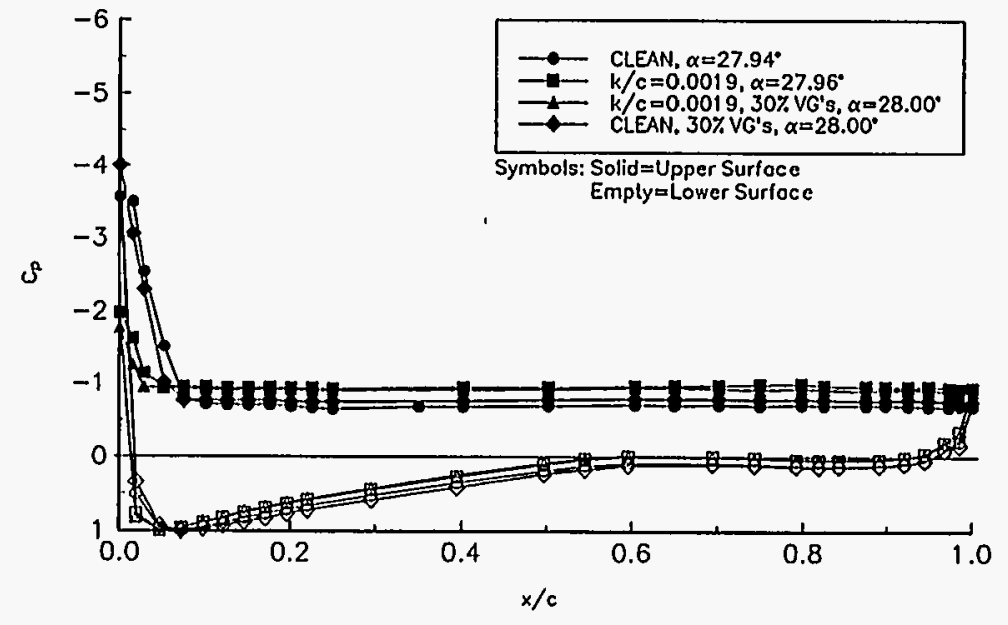

Figure 155. $\alpha=28^{\circ}$ 
$C_{p}$ VERSUS $x / c$

$\operatorname{LS}(1) 0417 \mathrm{MOO}\left(R_{\mathrm{e}}=1.5 \times 10^{6}\right)$

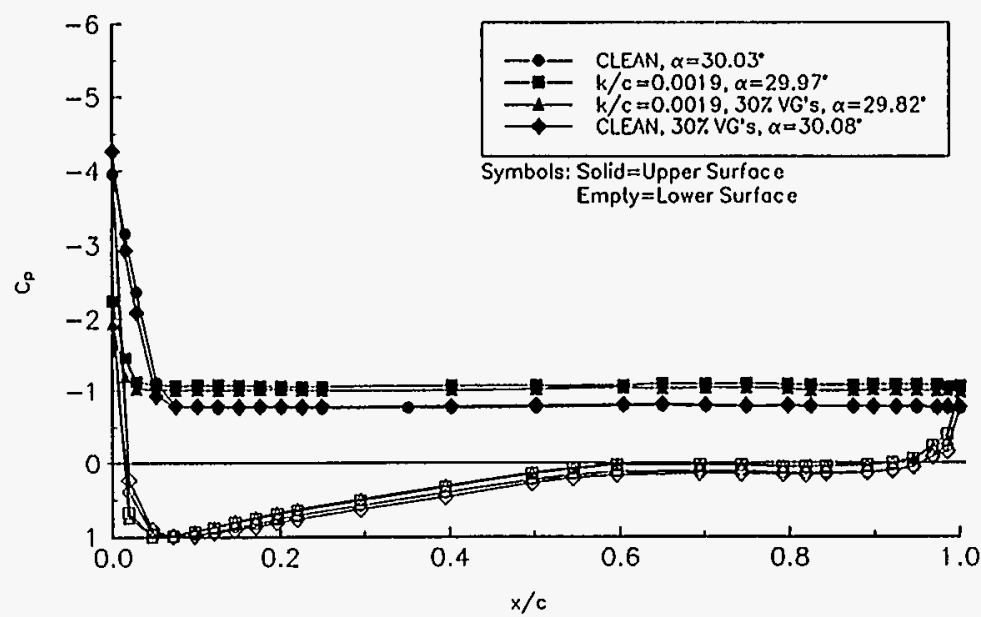

Figure 156. $\alpha=30^{\circ}$

$C_{p}$ VERSUS $x / c$

$\operatorname{LS}(1) 0417 \operatorname{MOD}\left(R_{0}=1.5 \times 10^{6}\right)$

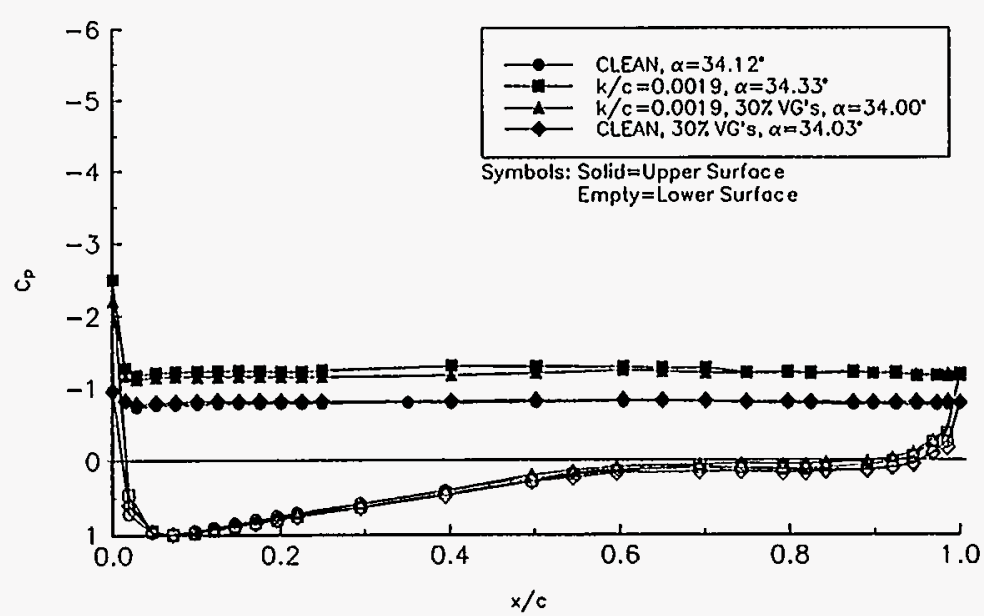

Figure 158. $\alpha=34^{\circ}$
$C_{0}$ VERSUS $x / c$

$\operatorname{LS}(1) 0417 \mathrm{MOD}\left(R_{\mathrm{B}}=1.5 \times 10^{6}\right)$

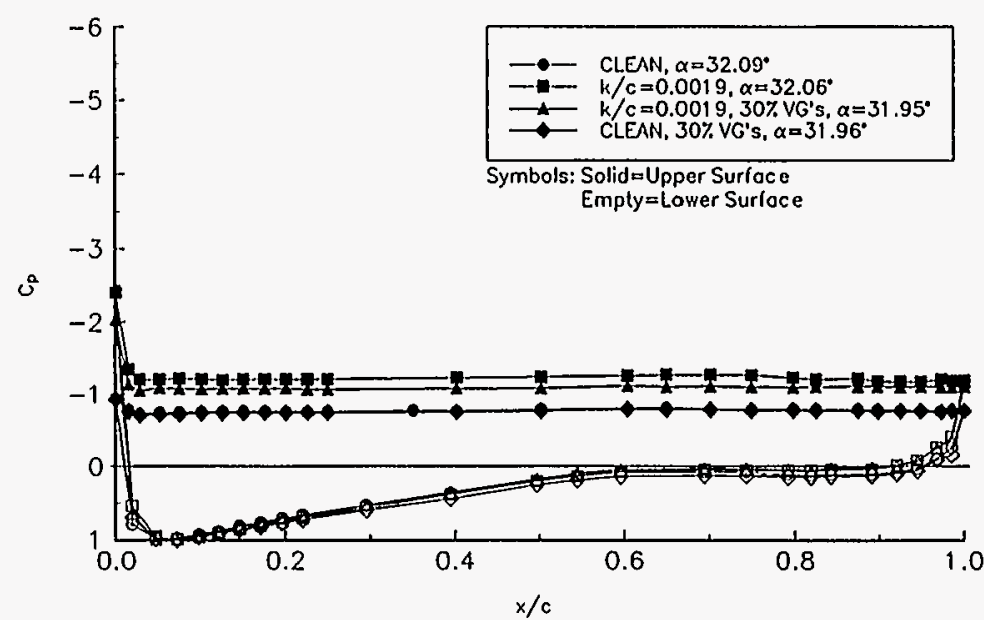

Figure 157. $\alpha=32^{\circ}$

$C_{p}$ VERSUS $x / c$

$\operatorname{LS}(1) 0417 \mathrm{MOD}\left(R_{e}=1.5 \times 10^{6}\right)$

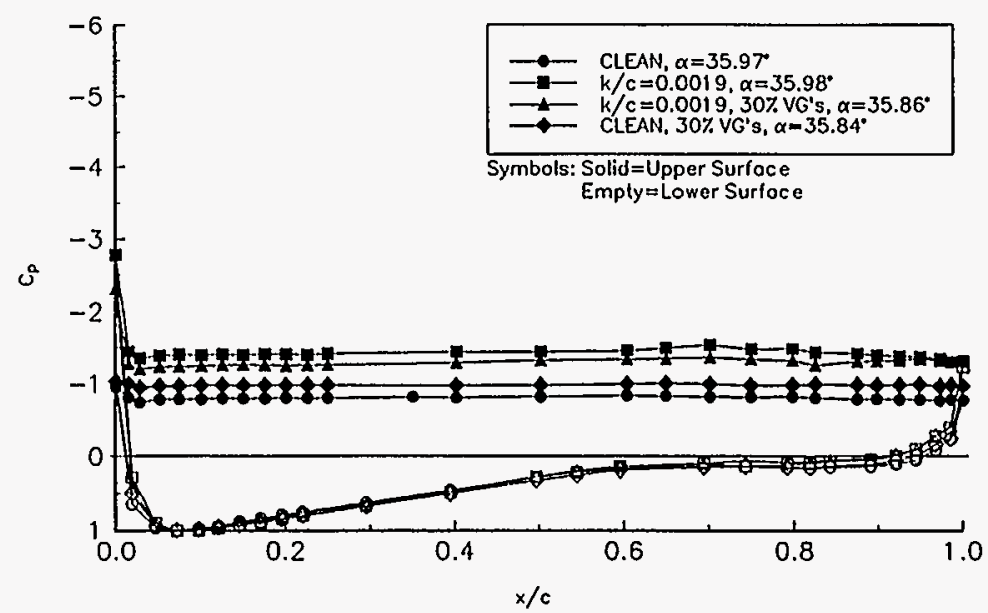

Figure 159. $\alpha=36^{\circ}$ 
$C_{p}$ VERSUS $x / c$

$\operatorname{LS}(1) 0417 \mathrm{MOD}\left(R_{\mathrm{B}}=1.5 \times 10^{6}\right)$

s

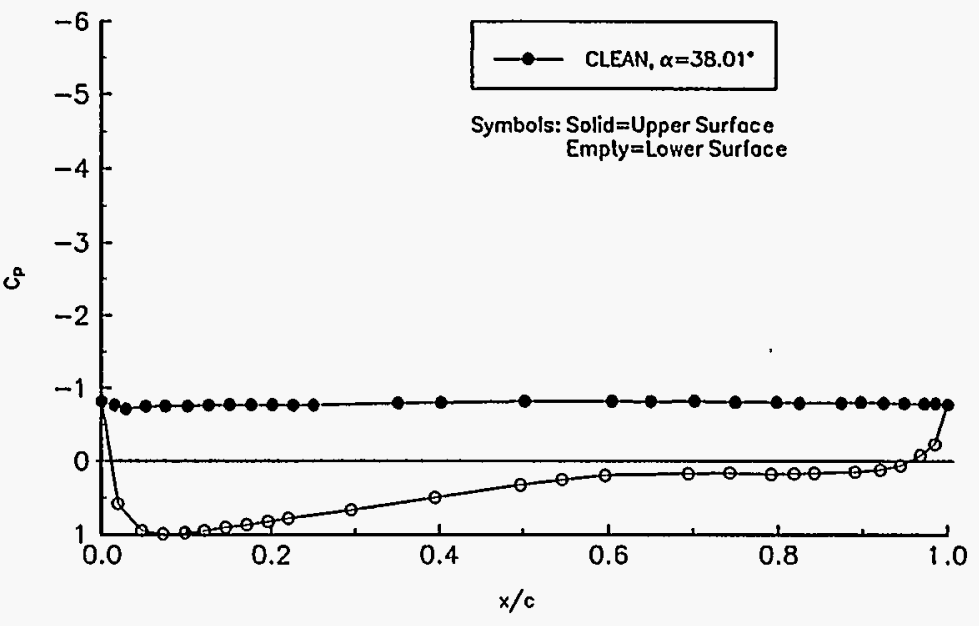

Figure 160. $\alpha=38^{\circ}$
C V VERSUS $x / c$

$\operatorname{LS}(1) 0417 \mathrm{MOD}\left(R_{\mathrm{B}}=1.5 \times 10^{6}\right)$

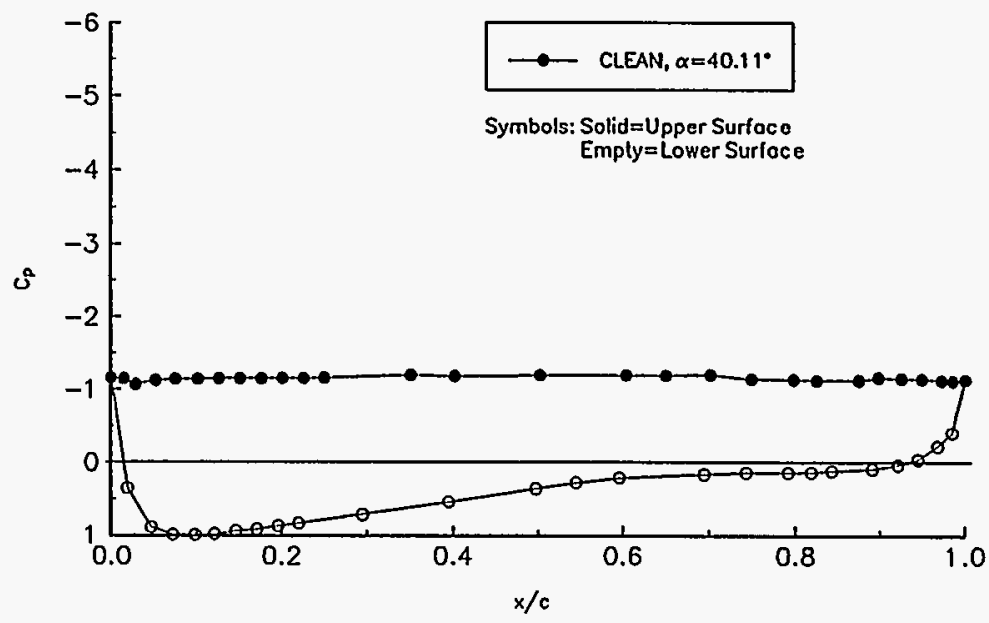

Figure 161. $\alpha=40^{\circ}$ 
Steady State Pressure Distributions

$$
\operatorname{Re}=\mathbf{2} .0 \text { million }
$$


$C_{p}$ VERSUS $x / c$

NASA LS(1)-0417MOD $\left(R_{e}=2.0 \times 10^{6}\right)$

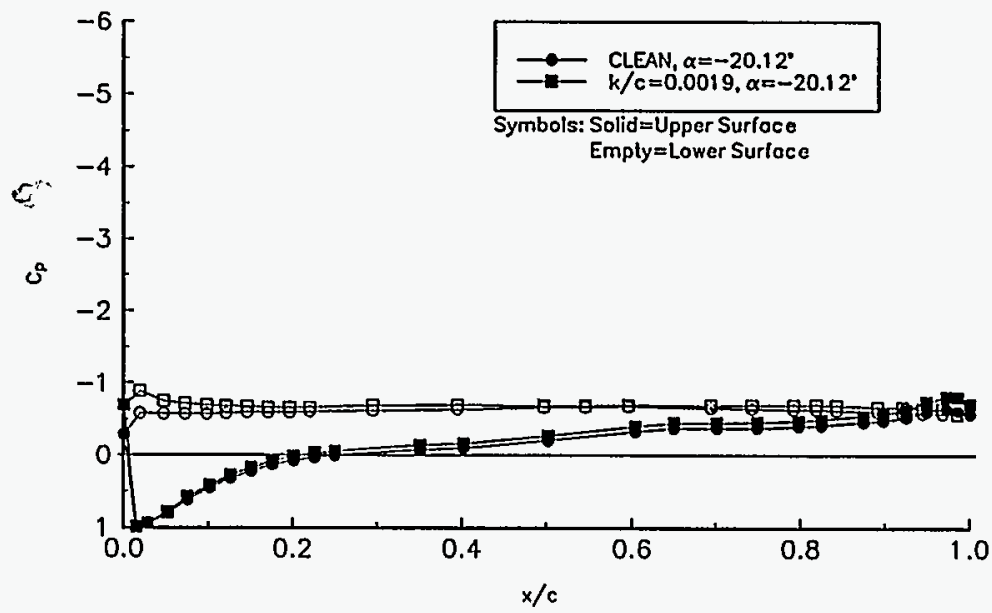

Figure 162. $\alpha=-20^{\circ}$

$C_{p}$ VERSUS $x / c$

NASA LS(1)-0417 MOD $\left(R_{\theta}=2.0 \times 10^{6}\right)$

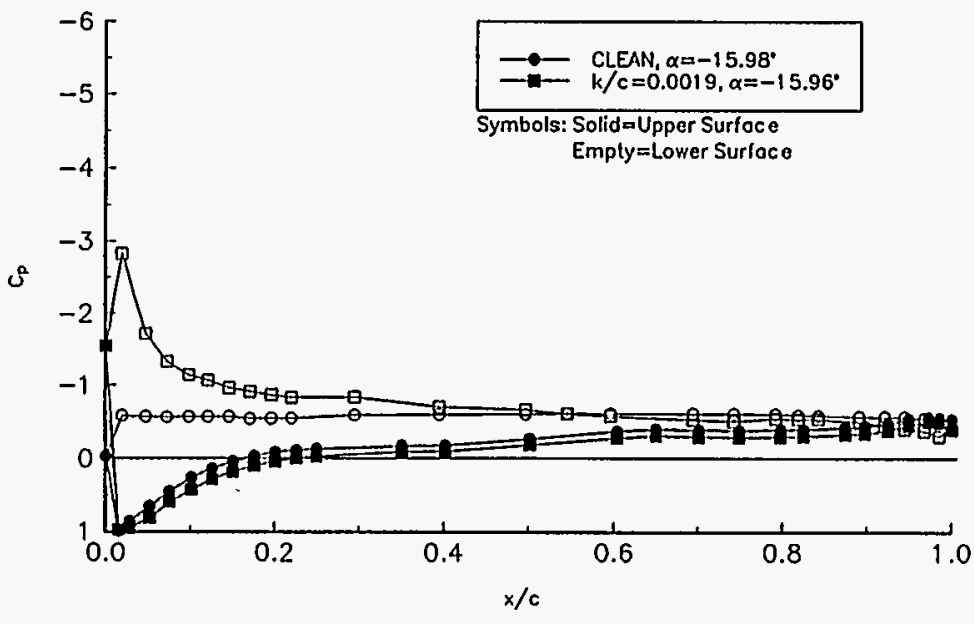

Figure 164. $\alpha=-16^{\circ}$
NASALS(1) $-0417 \mathrm{MOD}\left(R_{\mathrm{B}}=2.0 \times 10^{6}\right)$

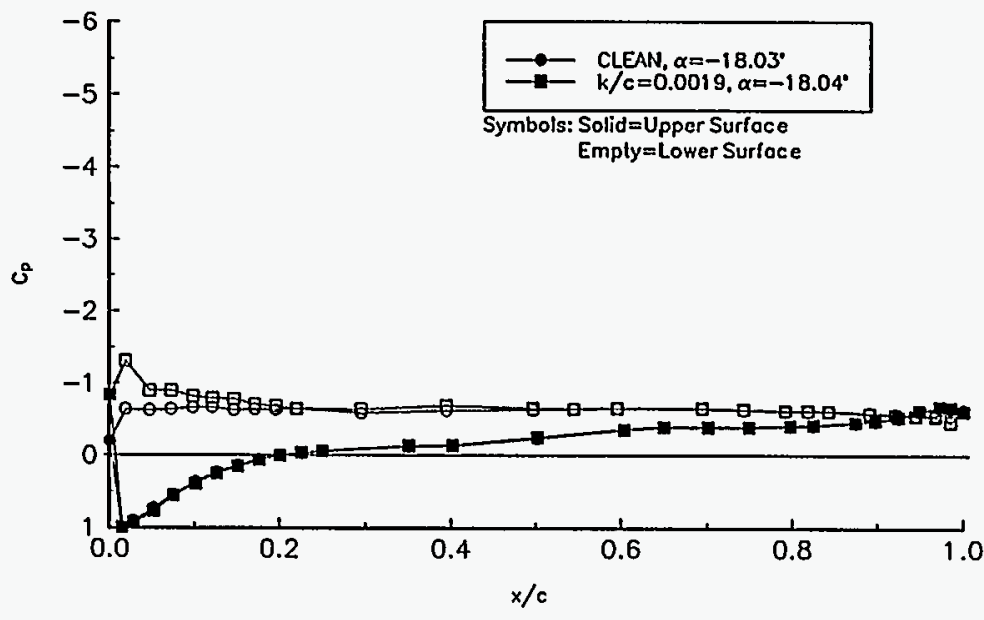

Figure 163. $\alpha=-18^{\circ}$

$C_{p}$ VERSUS $x / c$

NASA LS(1)-0417 MOD $\left(R_{e}=2.0 \times 10^{8}\right)$

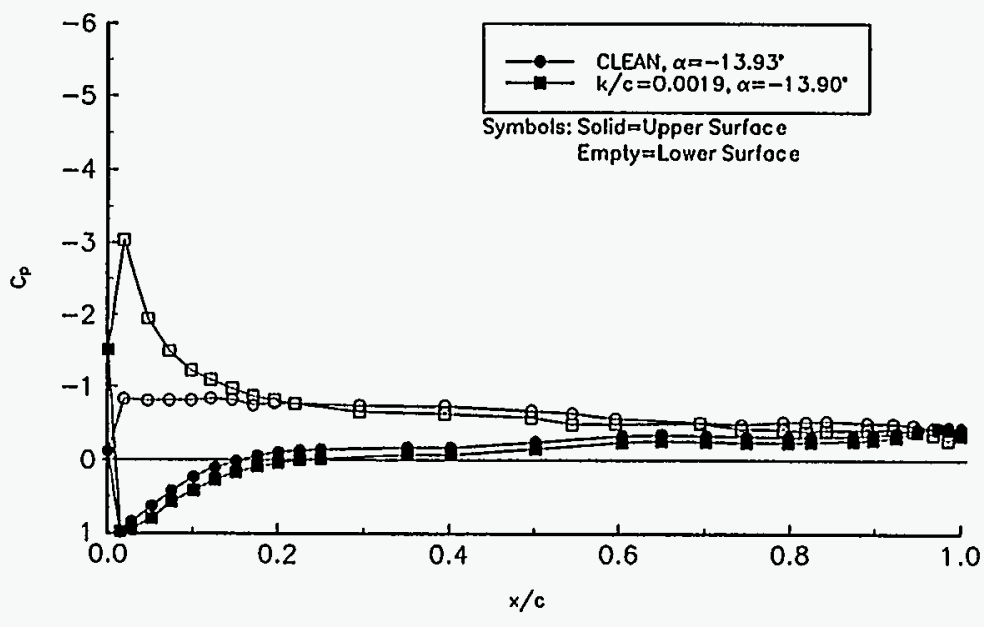

Figure 165. $\alpha=-14^{\circ}$ 
$C_{p}$ VERSUS $x / c$

NASA LS $(1)-0417 \mathrm{MOD}\left(R_{0}=2.0 \times 10^{8}\right)$

o

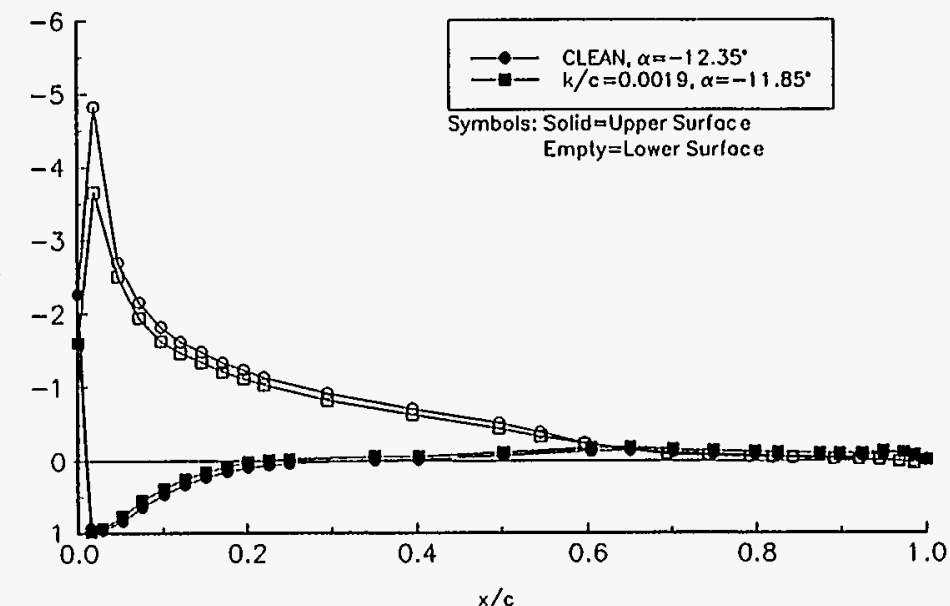

Figure 166. $\alpha=-12^{\circ}$

C VERSUS $x / c$

NASA LS (1) $-0417 \mathrm{MOD}\left(R_{e}=2.0 \times 10^{6}\right)$

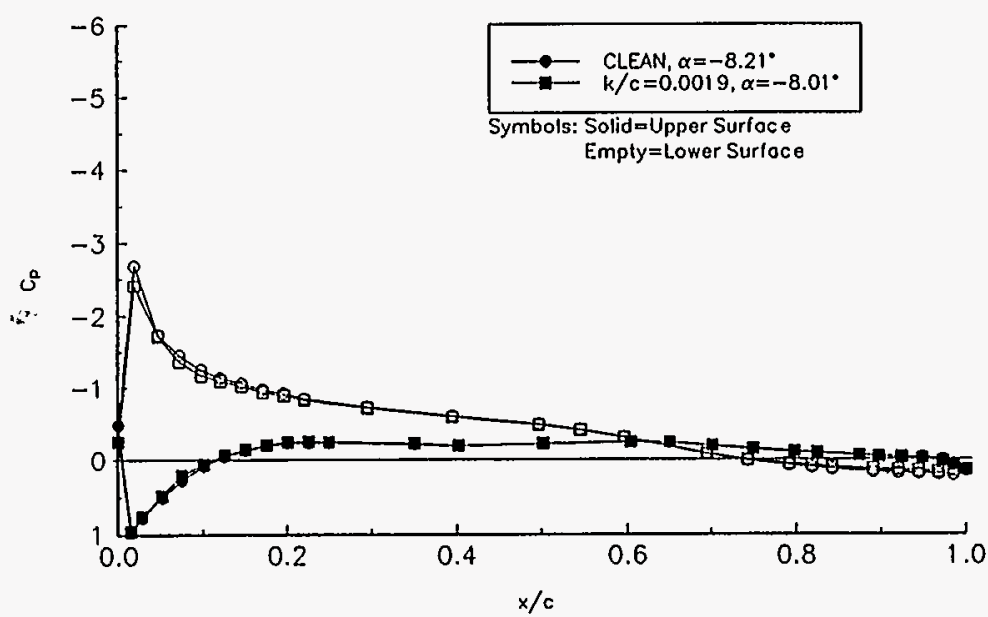

Figure 168. $\alpha=-8^{\circ}$
$\mathrm{C}_{\mathrm{p}}$ VERSUS $\mathrm{x} / \mathrm{c}$

NASA LS(1)-0417MOD $\left(R_{e}=2.0 \times 10^{8}\right)$

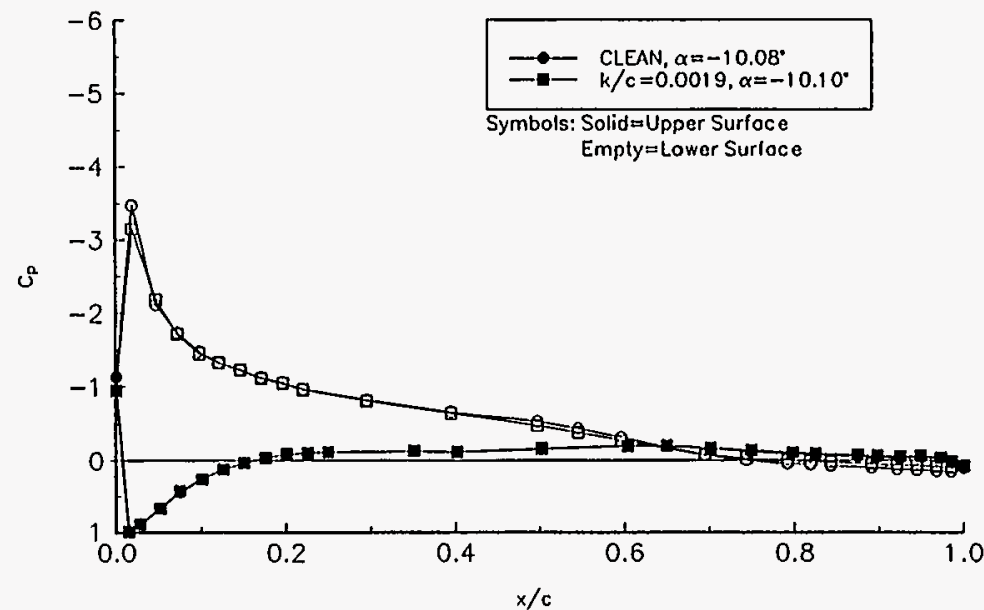

Figure 167. $\alpha=-10^{\circ}$

$C_{p}$ VERSUS $x / c$

NASA LS $(1)-0417$ MOD $\left(R_{\theta}=2.0 \times 10^{\circ}\right)$

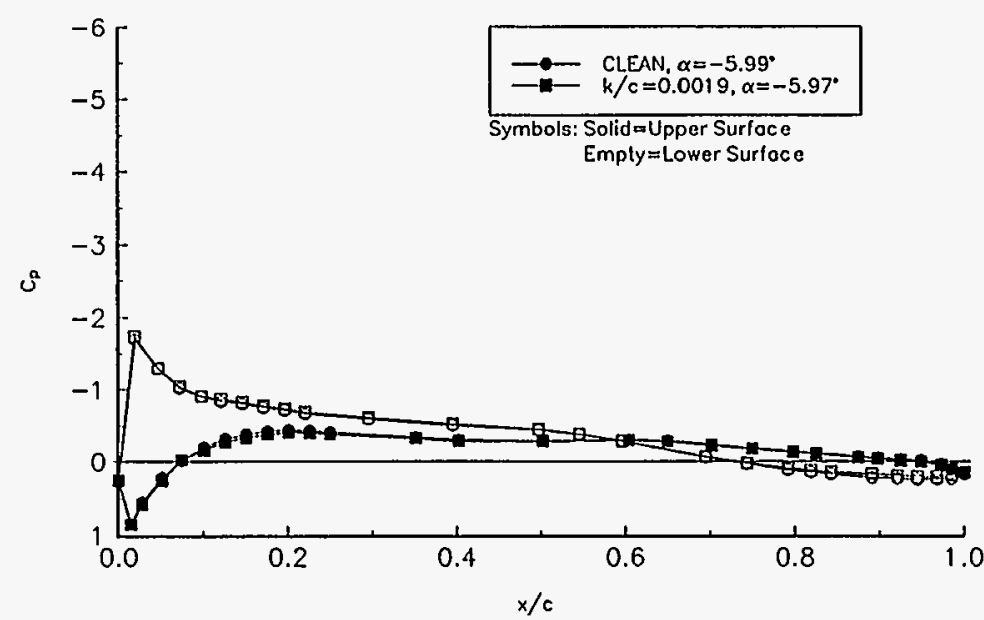

Figure 169. $\alpha=-6^{\circ}$ 
$C_{p}$ VERSUS $x / c$

NASALS(1)-0417 MOD $\left(R_{0}=2.0 \times 10^{6}\right)$

o

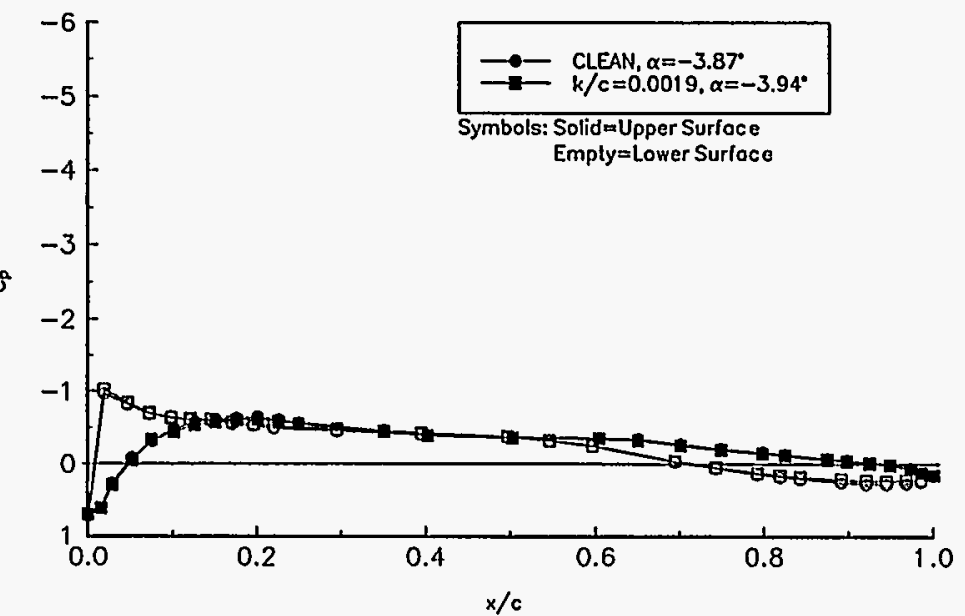

Figure 170. $\alpha=-4^{\circ}$

$\mathrm{C}_{\mathrm{p}}$ VERSUS $x / \mathrm{c}$

NASA LS (1)-0417 MOD $\left(R_{\mathrm{e}}=2.0 \times 10^{6}\right)$

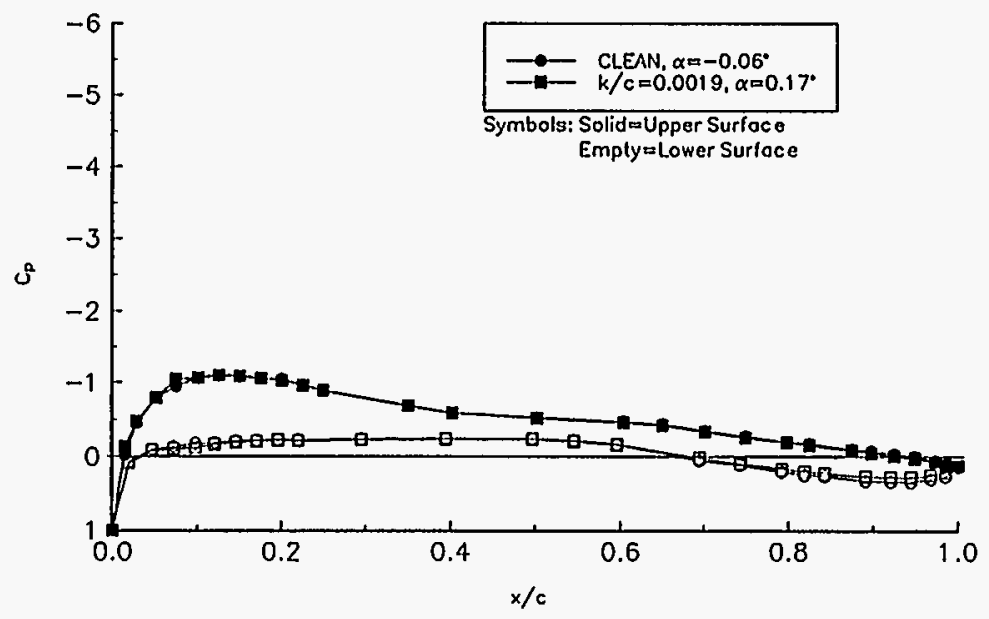

Figure 172. $\alpha=0^{\circ}$
$C_{p}$ VERSUS $x / C$

NASA LS(1) -0417 MOD $\left(R_{e}=2.0 \times 10^{8}\right)$

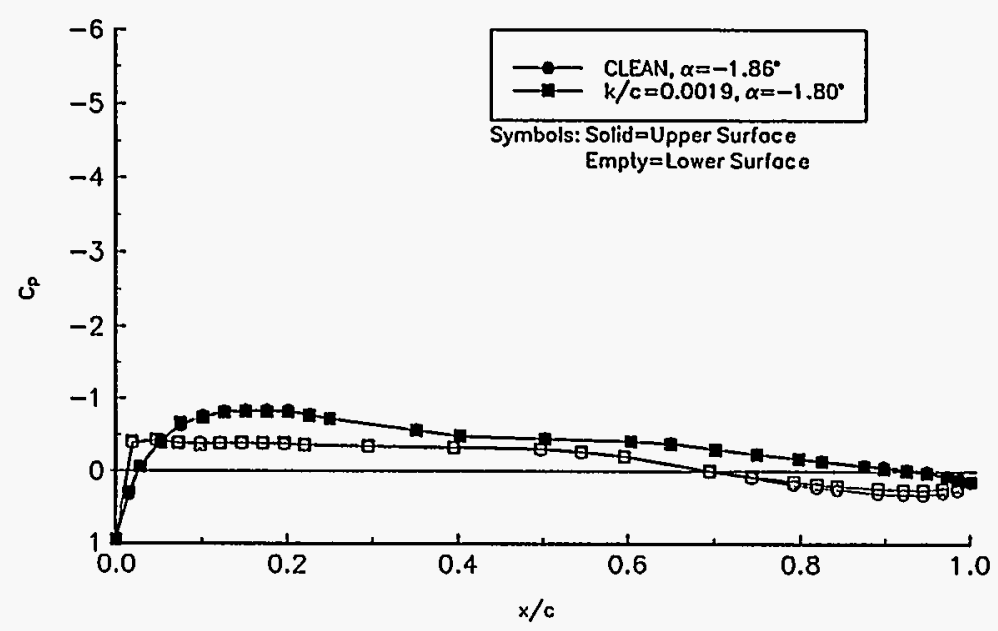

Figure 171. $\alpha=-2^{\circ}$

$C_{p}$ VERSUS $x / c$
NASA LS $(1)-0417 \mathrm{MOD}\left(R_{e}=2.0 \times 10^{\circ}\right)$

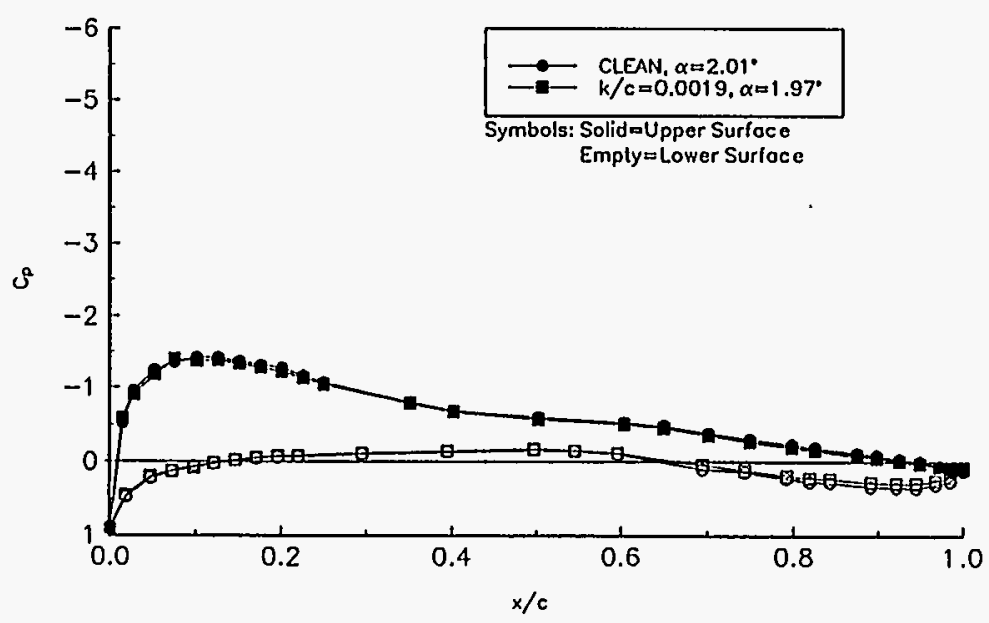

Figure 173. $\alpha=2^{\circ}$ 
$C_{p}$ VERSUS $x / c$

NASA LS $(1)-0417 \mathrm{MOD}\left(R_{0}=2.0 \times 10^{\circ}\right)$

o

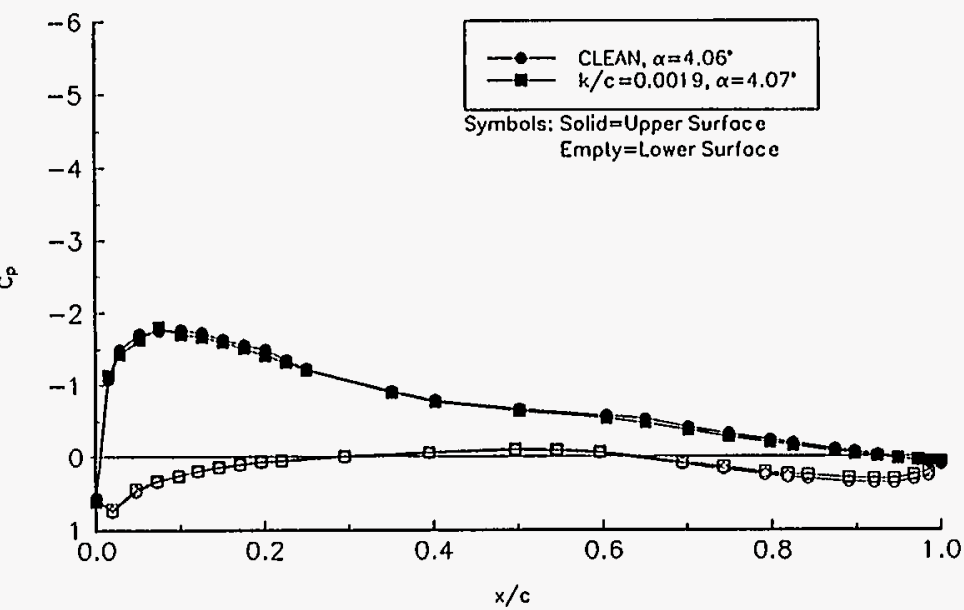

Figure 174. $\alpha=4^{\circ}$

$C_{p}$ VERSUS $x / c$

NASALS(1) $-0417 \mathrm{MOD}\left(R_{\mathrm{e}}=2.0 \times 10^{6}\right)$

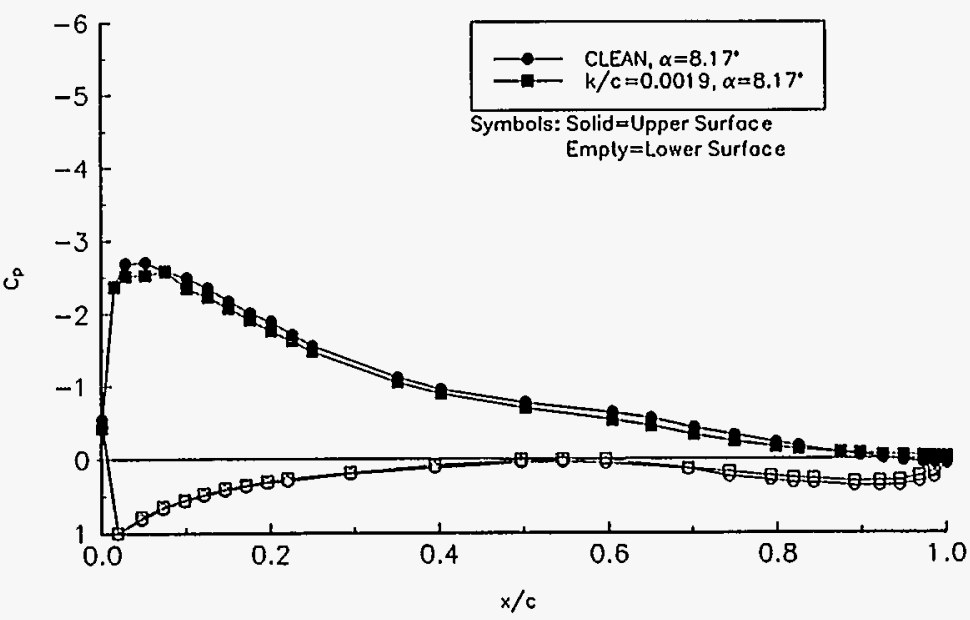

Figure 176. $\alpha=8^{\circ}$
Cp VERSUS $x$

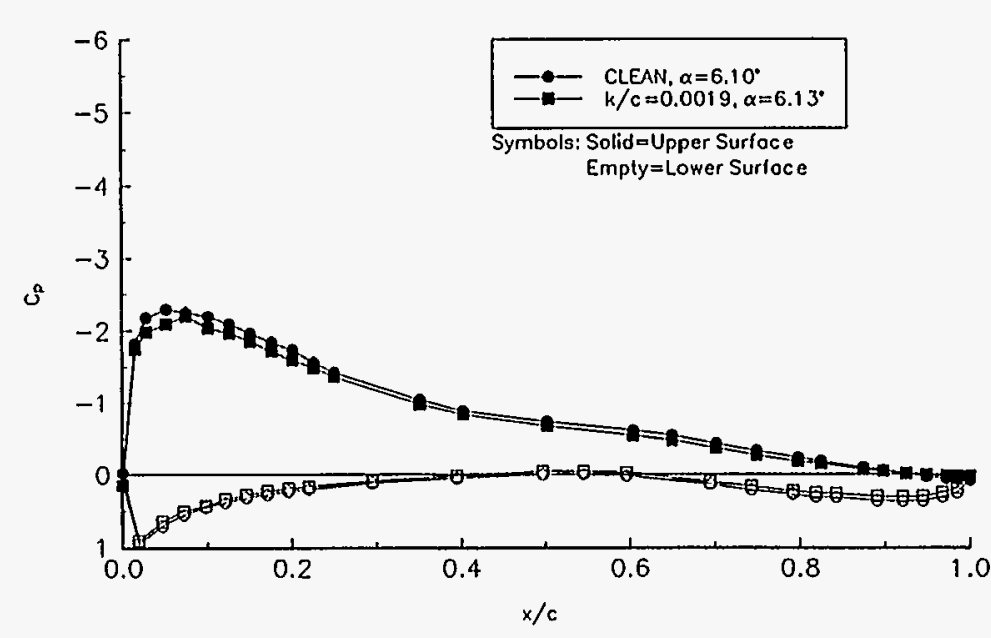

Figure 175. $\alpha=6^{\circ}$

VERSUS $x / \mathrm{c}$

NASA LS (1)-0417 MOD $\left(R_{e}=2.0 \times 10^{6}\right)$

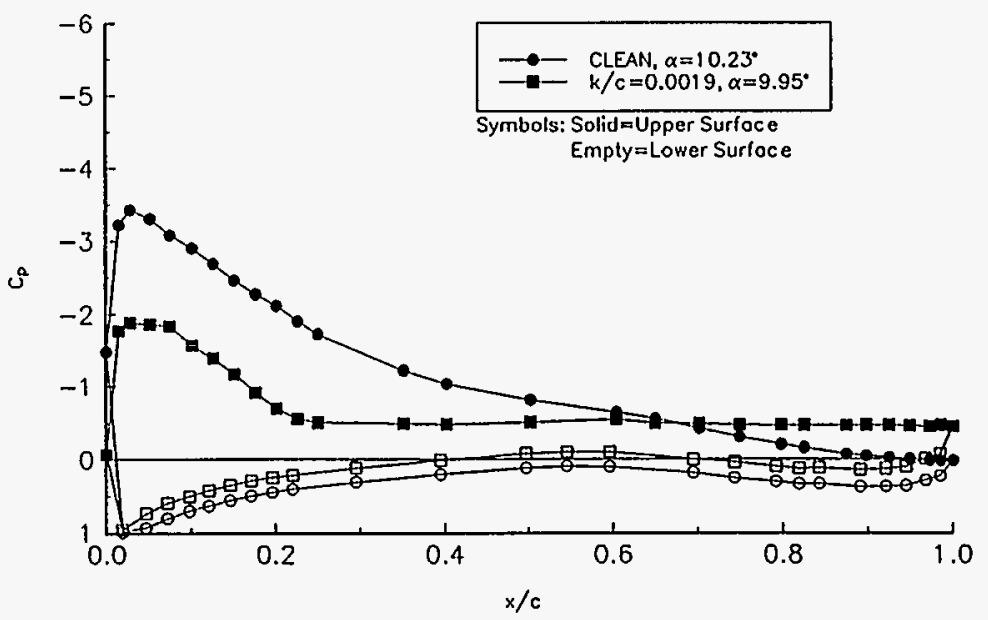

Figure 177. $\alpha=10^{\circ}$ 
$C_{p}$ VERSUS $x / c$

NASA LS(1)-0417 MOD $\left(R_{c}=2.0 \times 10^{6}\right)$

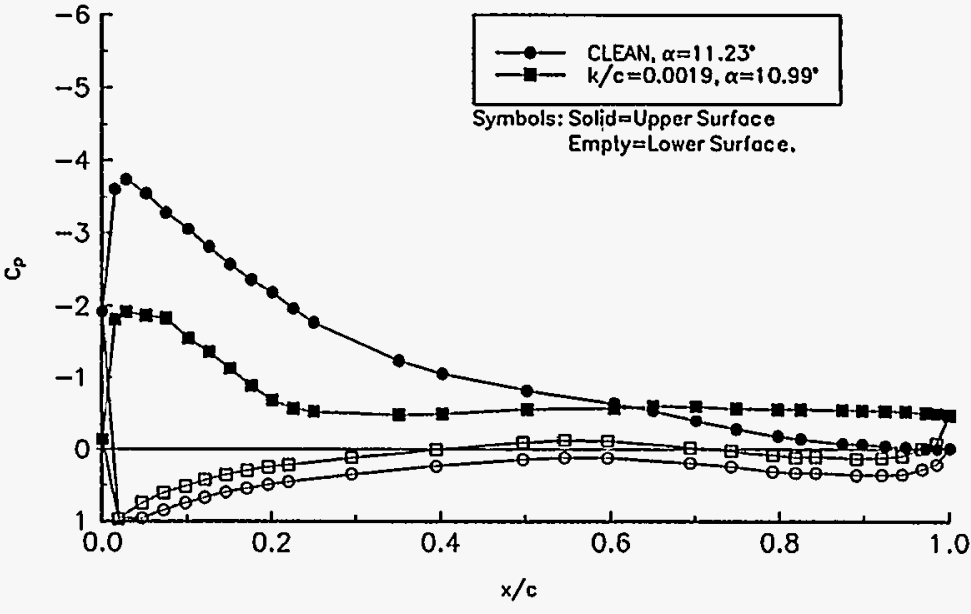

Figure 178. $\alpha=11^{\circ}$

$C_{p}$ VERSUS $x / c$

$\operatorname{NASALS}(1)-0417 \mathrm{MOD}\left(R_{\mathrm{a}}=2.0 \times 10^{\circ}\right)$

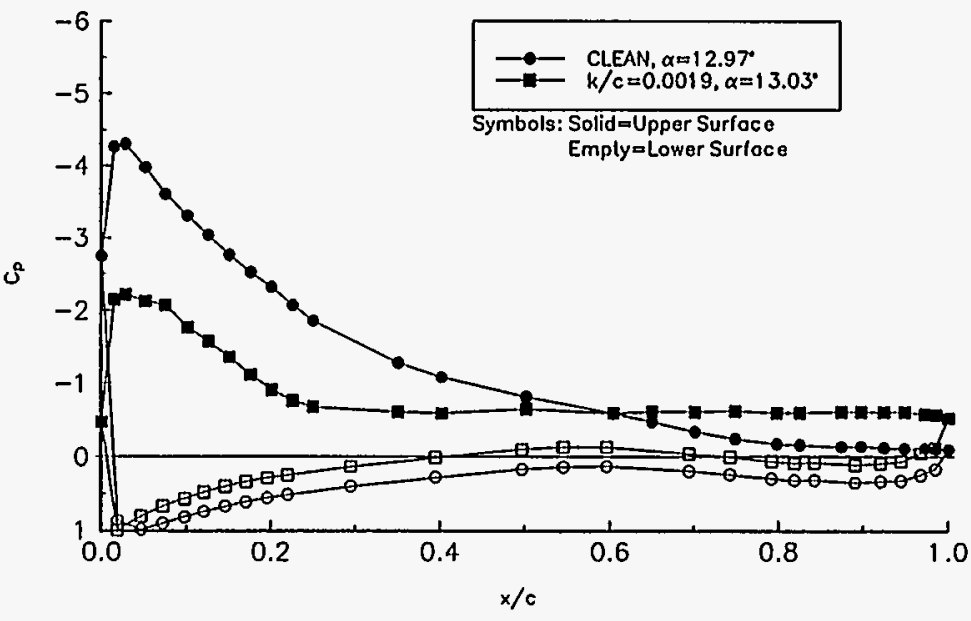

Figure 180. $\alpha=13^{\circ}$
C VERSUS $x / c$

ASA LS $(1)-0417 \mathrm{MOD}\left(R_{0}=2.0 \times 10^{5}\right)$

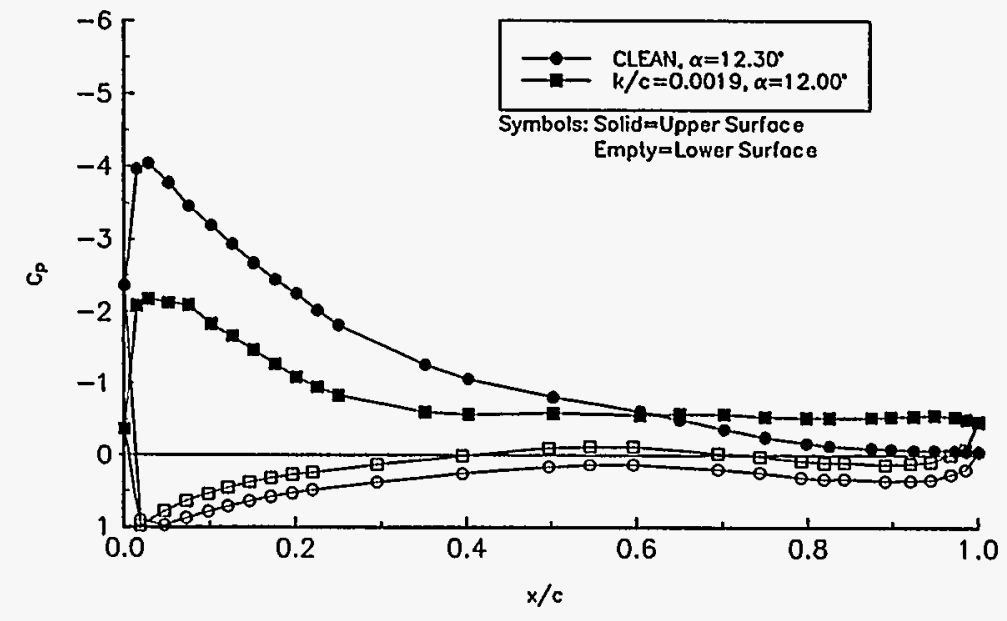

Figure 179. $\alpha=12^{\circ}$

$C_{p}$ VERSUS $x / c$

$\operatorname{NASALS}(1)-0417 \mathrm{MOD}\left(R_{0}=2.0 \times 10^{\circ}\right)$

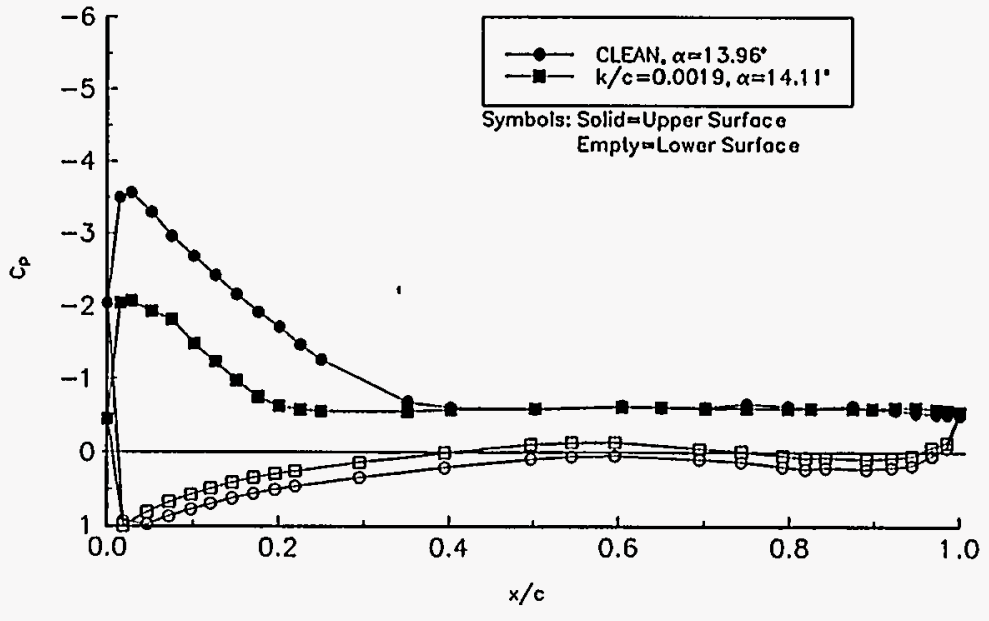

Figure 181. $\alpha=14^{\circ}$ 
$C_{p}$ VERSUS $x / c$

NASA LS $(1)-0417$ MOD $\left(R_{\mathrm{e}}=2.0 \times 10^{\circ}\right)$

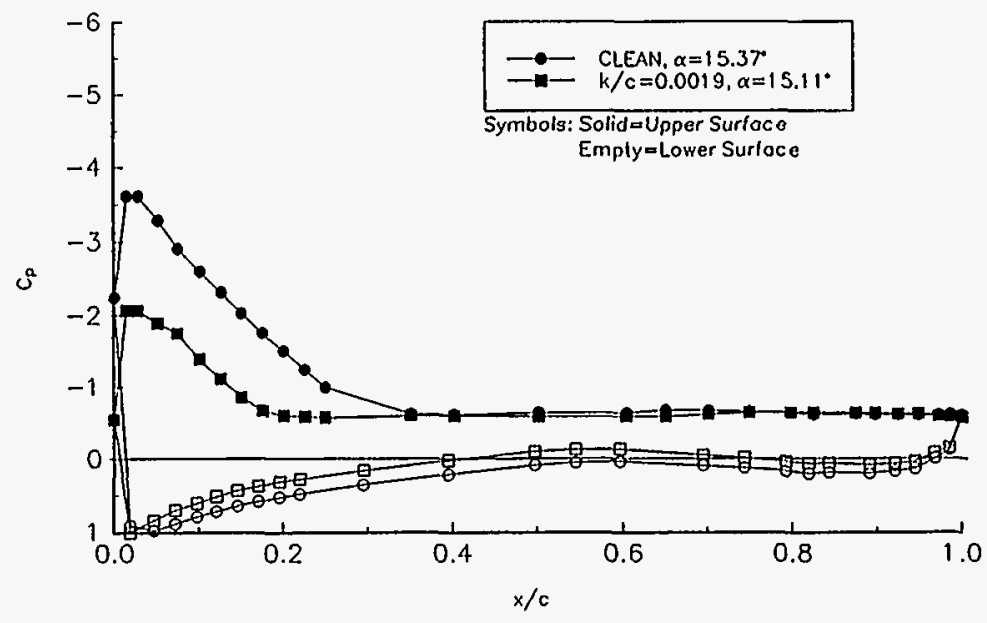

Figure 182. $\alpha=15^{\circ}$

$C_{p}$ VERSUS $x / c$

NASALS(1)-0417MOD $\left(R_{Q}=2.0 \times 10^{6}\right)$

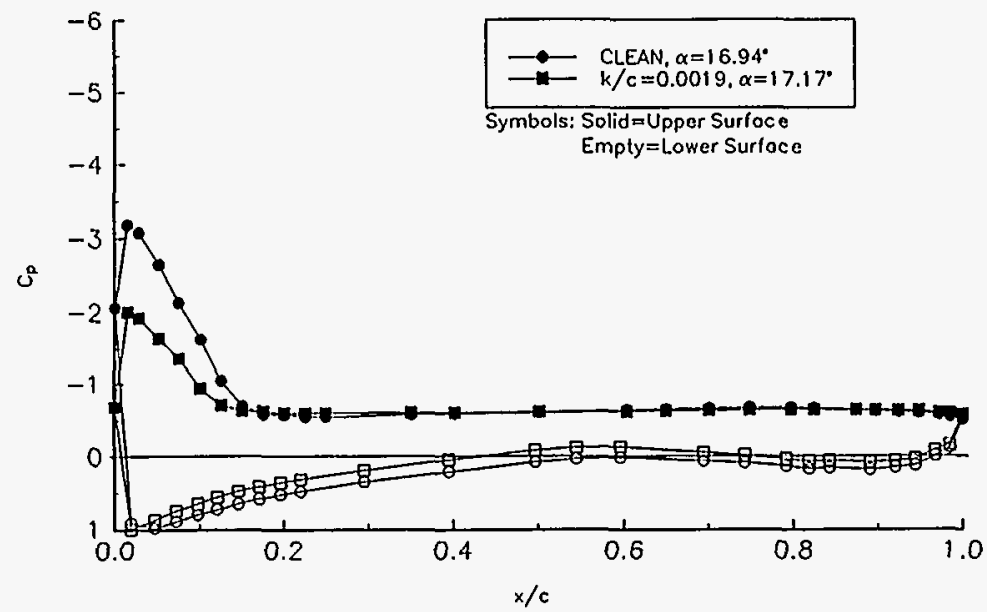

Figure 184. $\alpha=17^{\circ}$
NASAIS(1) VOATSUS $X / C$

ASALS(1) $-0417 \mathrm{MOO}\left(R_{\mathrm{e}}=2.0 \times 10^{\circ}\right)$

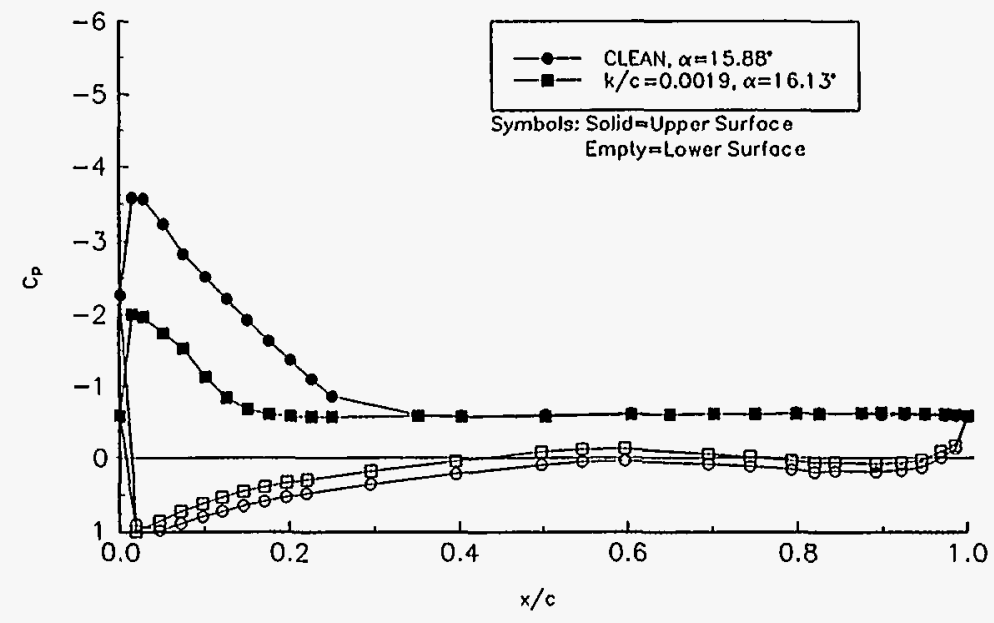

Figure 183. $\alpha=16^{\circ}$

$C_{p}$ VERSUS $x / c$ NASA LS $(1)-0417 \mathrm{MOO}\left(R_{0}=2.0 \times 10^{6}\right)$

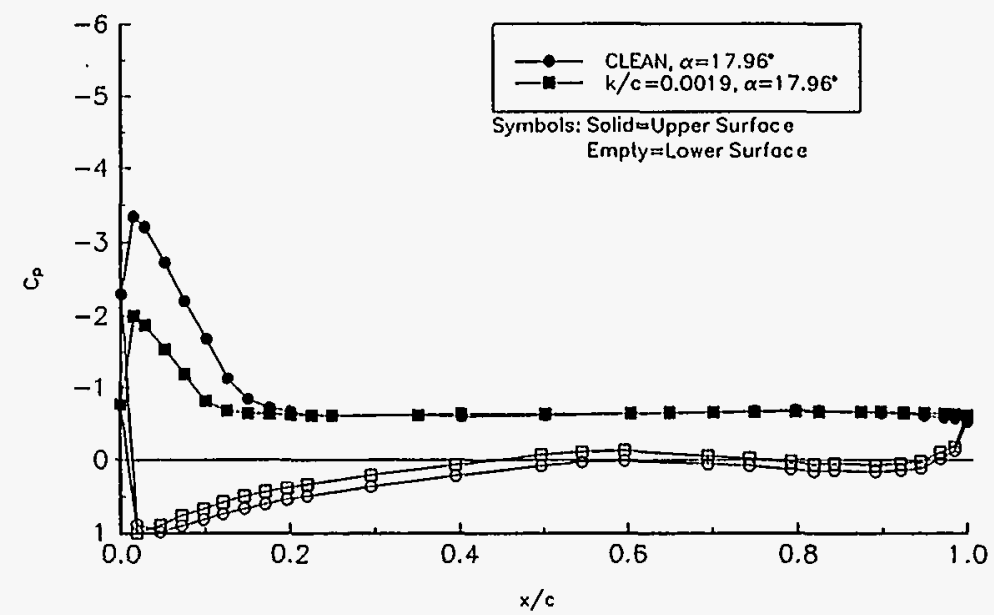

Figure 185. $\alpha=18^{\circ}$ 


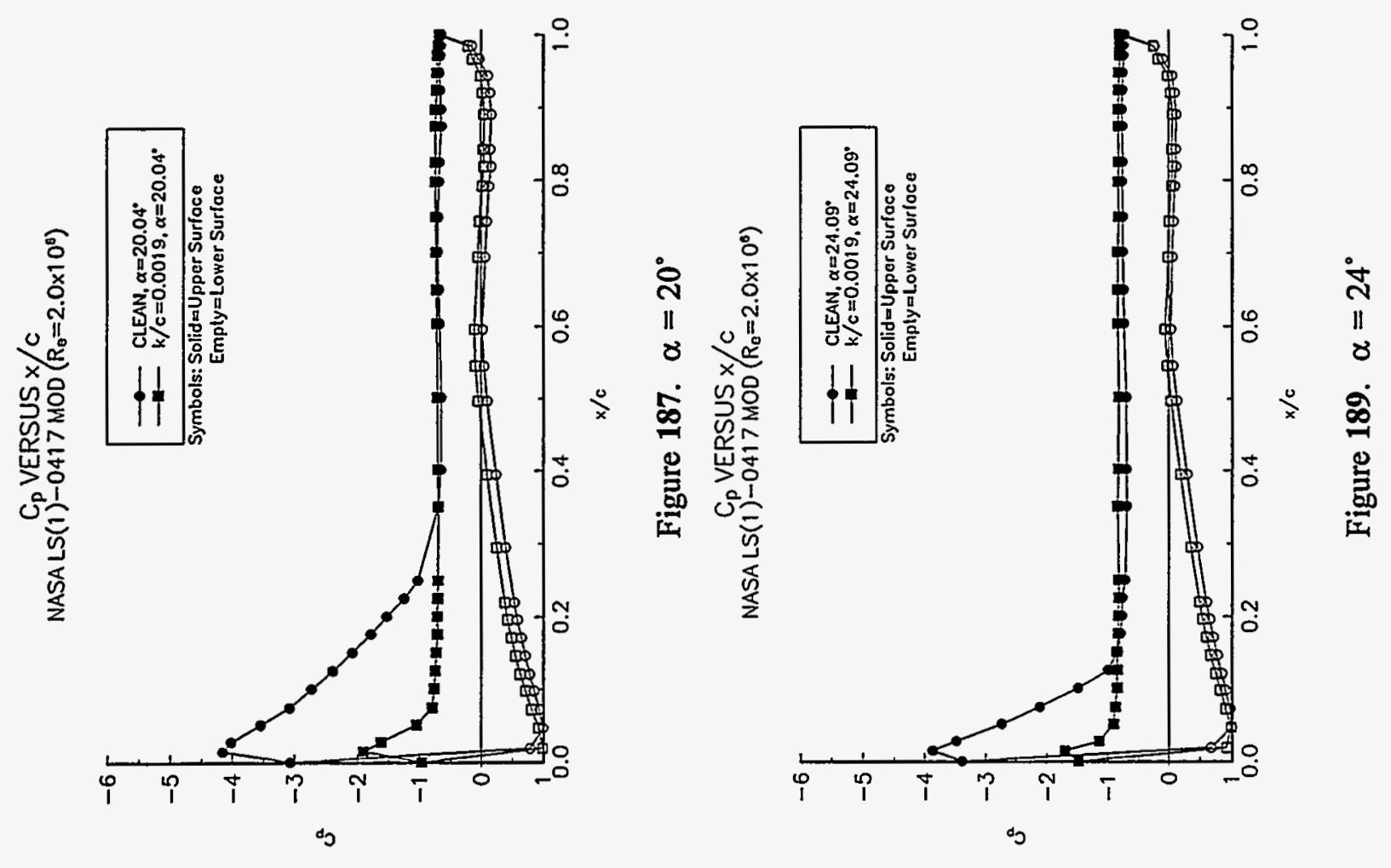

ळ

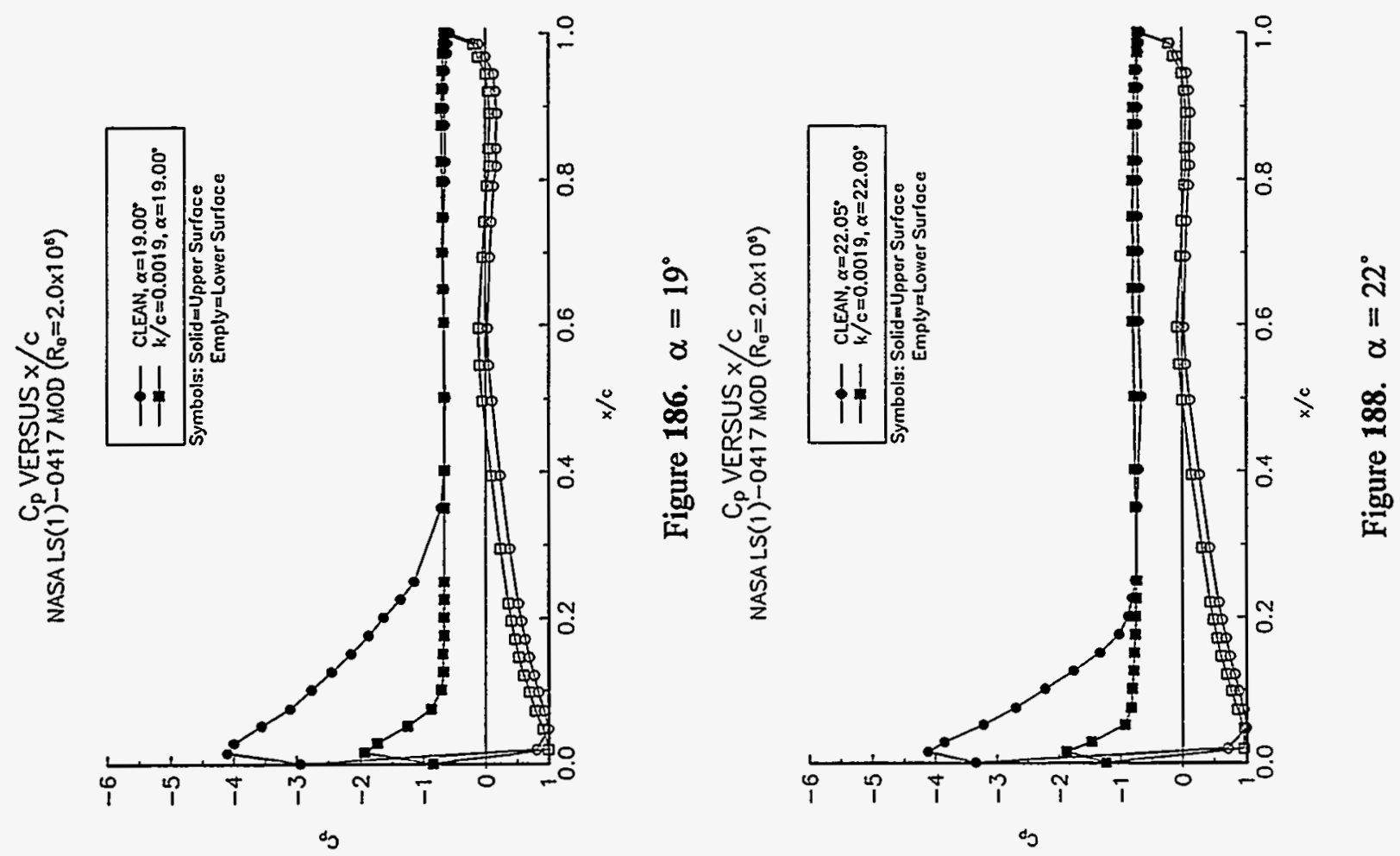


$C_{p}$ VERSUS $\times / C$
NASA LS $(1)-0417$ MOD $\left(R_{0}=2.0 \times 10^{6}\right)$

a

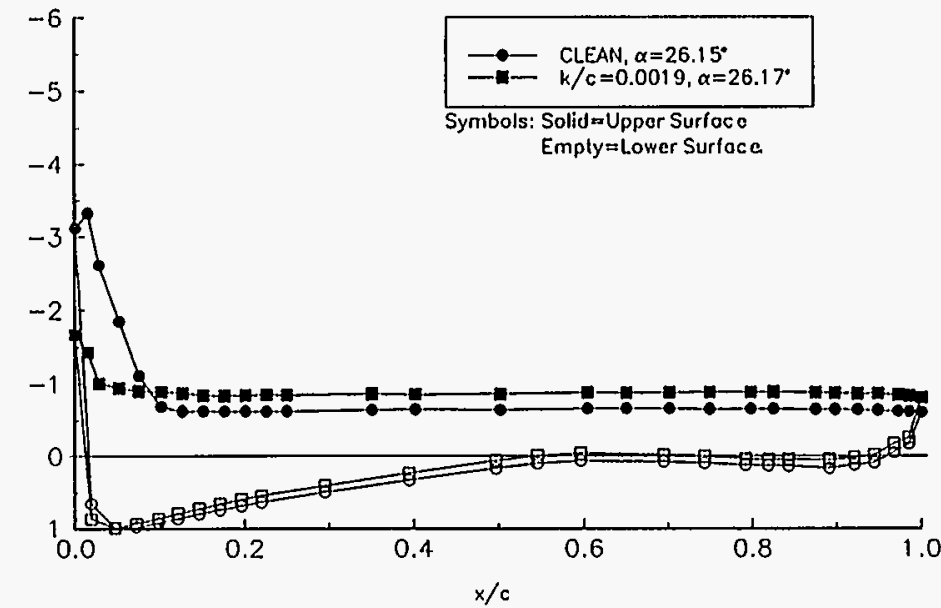

Figure 190. $\alpha=26^{\circ}$

$\mathrm{C}_{\mathrm{p}}$ VERSUS $\times / \mathrm{c}$

NASA LS $(1)-0417 \mathrm{MOD}\left(R_{B}=2.0 \times 10^{\circ}\right)$

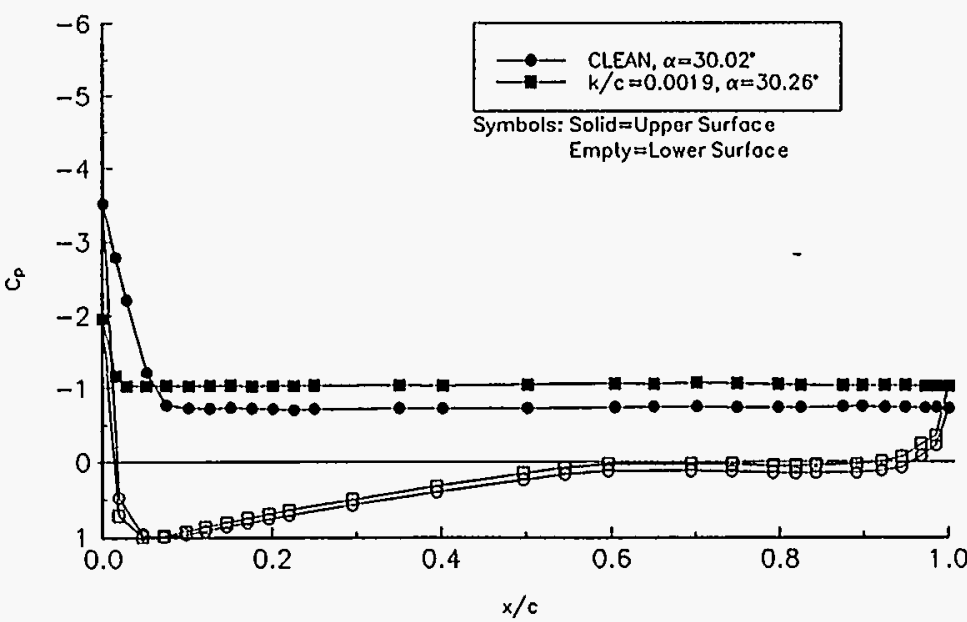

Figure 192. $\alpha=30^{\circ}$
$C_{p}$ VERSUS $x / c$

NASA LS(1)-0417 MOD $\left(R_{c}=2.0 \times 10^{6}\right)$

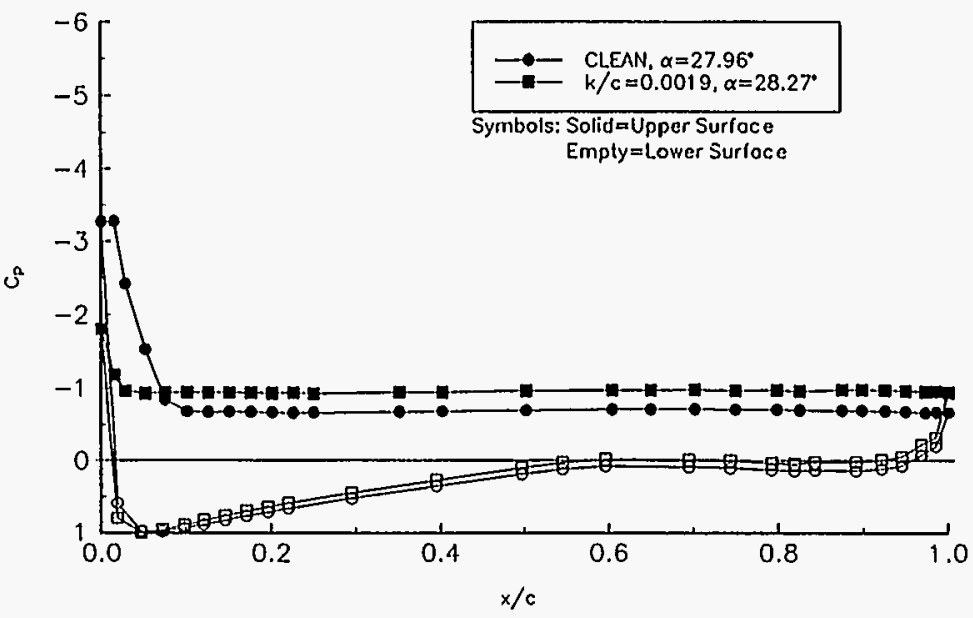

Figure 191. $\alpha=28^{\circ}$

$C_{p}$ VERSUS $x / c$

NASALS(1)-0417 MOD $\left(R_{0}=2.0 \times 10^{6}\right)$

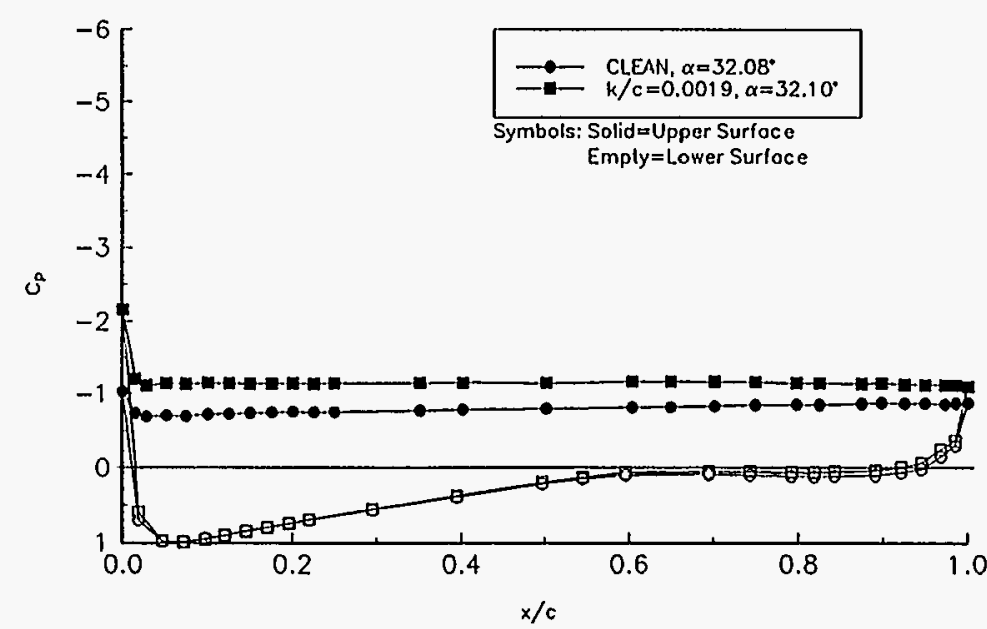

Figure 193. $\alpha=32^{\circ}$ 
$\mathrm{C}_{\mathrm{p}}$ VERSUS $\times / \mathrm{c}$

NASA LS (1) $-0417 \mathrm{MOD}\left(R_{Q}=2.0 \times 10^{6}\right)$

s

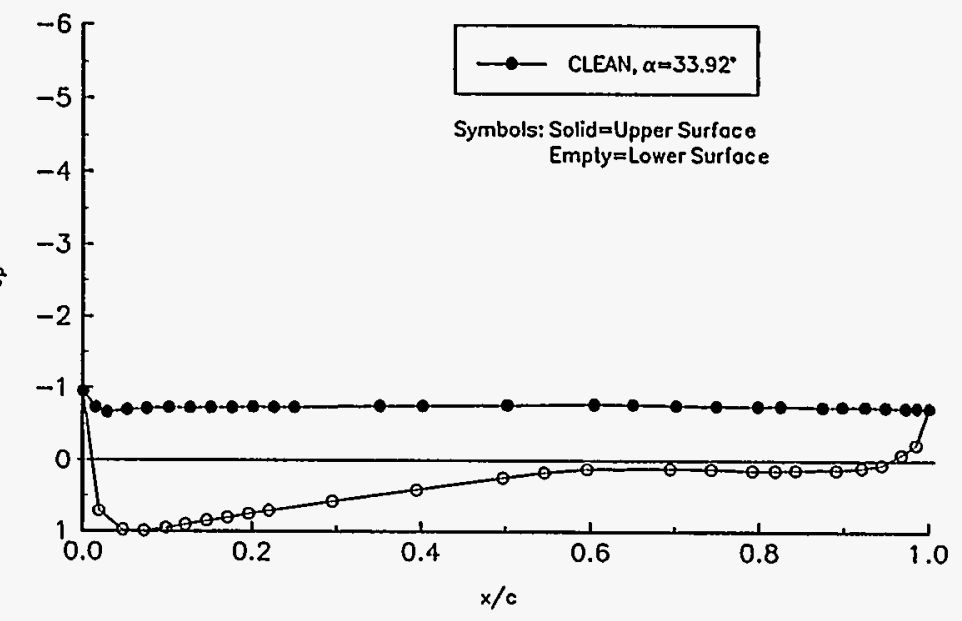

Figure 194. $\alpha=34^{\circ}$

$C_{p}$ VERSUS $x / c$

NASA LS $(1)-0417 \mathrm{MOD}\left(R_{e}=2.0 \times 10^{6}\right)$

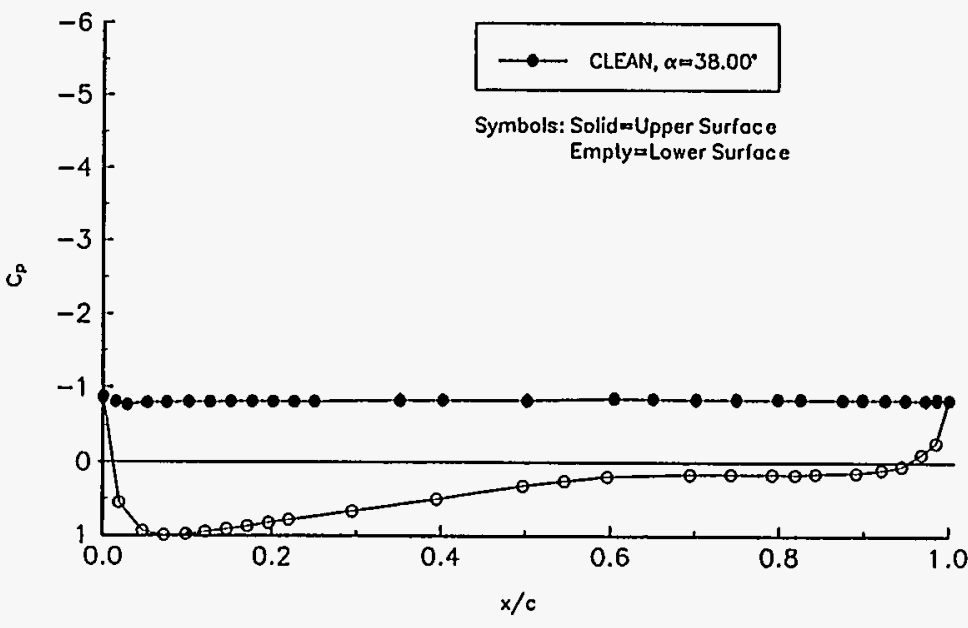

Figure 196. $\alpha=38^{\circ}$

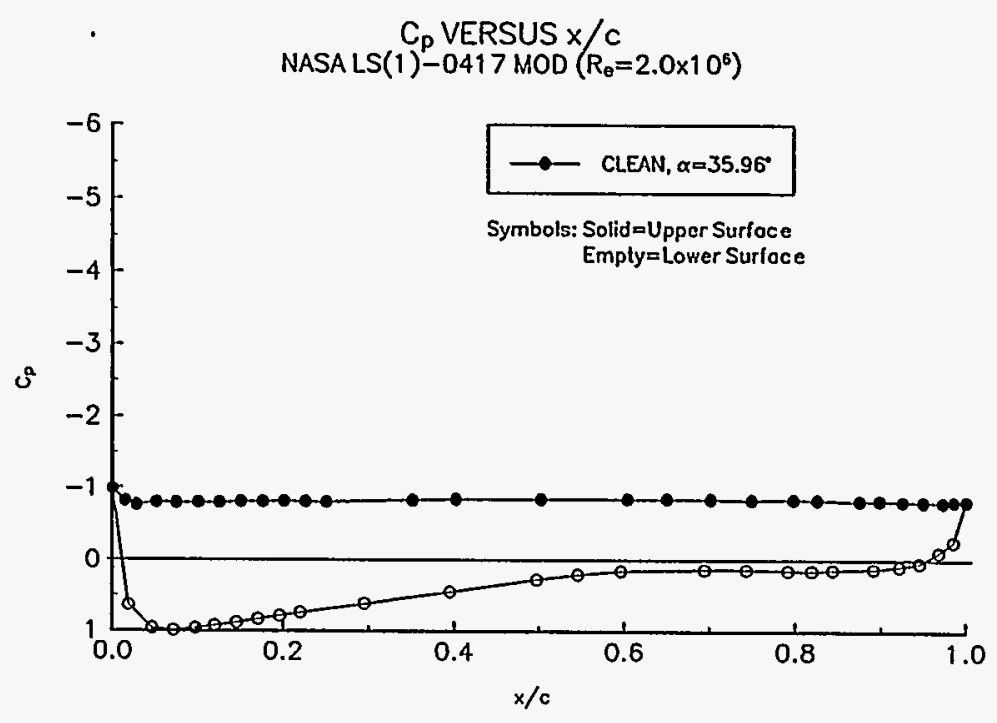

Figure 195. $\alpha=36^{\circ}$

$\mathrm{C}_{\mathrm{p}}$ VERSUS $\times / \mathrm{c}$

NASA LS $(1)-0417 \mathrm{MOD}\left(R_{\mathrm{g}}=2.0 \times 10^{6}\right)$

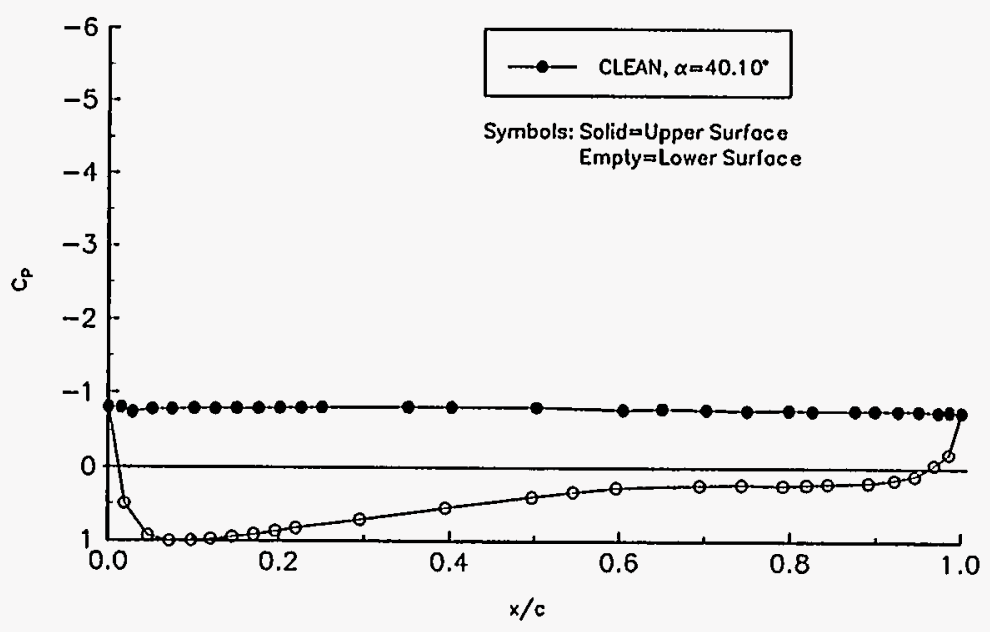

Figure 197. $\alpha=40^{\circ}$ 


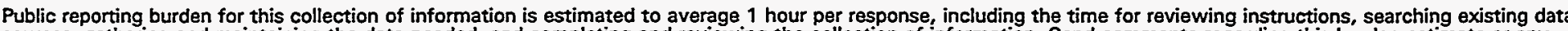

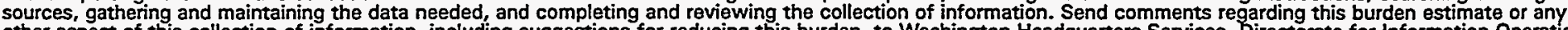

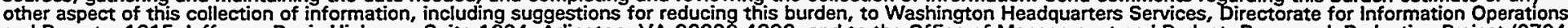

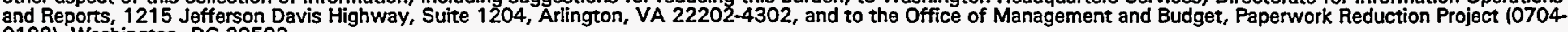
01881 , Washington, DC 20503.

\begin{tabular}{|l|l|l|}
\hline 1. & $\begin{array}{l}\text { 2. REPORT DATE } \\
\text { December 1995 }\end{array}$ & $\begin{array}{l}\text { 3. REPORT TYPE AND DATES COVERED } \\
\text { Subcontract Repoit }\end{array}$ \\
\hline
\end{tabular}

4. TITLE AND SUBTITLE

Effects of Surface Roughness and Vortex Generators on the LS(1)-0417MOD Airfoil

5. FUNDING NUMBERS

C:

TA: WE618120
8. PERFORMING ORGANIZATION REPORT NUMBER

7. PERFORMING ORGANIZATION NAME(S) AND ADDRESS(ES)

Dr. Gerald Gregorek

The Ohio State University

Aero \& Astronautical Research

2300 West Case Road

Columbus, Ohio 43220

(614) 292-5491

9. SPONSORING/MONITORING AGENCY NAME(S) AND ADDRESS(ES)

National Renewable Energy Laboratory

1617 Cole Blvd.

Golden, CO 80401-3393

DE96000495

11. SUPPLEMENTARY NOTES

NREL Technical Monitor: C. P. Butterfield

12a. DISTRIBUTION/AVAILABILITY STATEMENT

National Technical Information Service

U.S. Department of Commerce

5285 Port Royal Road

Springfield, VA 22161

10. SPONSORING/MONITORING AGENCY REPORT NUMBER

13. ABSTRACT (Maximum 200 words)

An 18-inch constant-chord model of the LS(1)-0417MOD airfoil section was tested under two dimensional steady state conditions in the Ohio State University $7 \times 10$ Subsonic Wind Tunnel. The objective was to document section lift and moment characteristics under various model and air flow conditions. Surface pressure data was acquired at $-60^{\circ}$ through $+230^{\circ} \mathrm{geometric}$ angles of attack, at a nominal 1 million Reynolds number. Cases with and without leading edge grit roughness were investigated. The leading edge roughness simulated blade conditions in the field. Additionally, surface pressure data were acquired for Reynolds numbers of 1.5 and 2.0 million, with and without leading edge grit roughness; the angle of attack was limited to a $-20^{\circ}$ to $40^{\circ}$ range.

In general, results showed lift curve slope sensitivities to Reynolds number and roughness. The maximum lift coefficient was reduced as much as $29 \%$ by leading edge roughness. Moment coefficient showed little sensitivity to roughness beyond $50^{\circ}$ angle of attack, but the expected decambering effect of a thicker boundary layer with roughness did show at lower angles.

Tests were also conducted with vortex generators located at the $30 \%$ chord location on the upper surface only, at 1 and 1.5 million Reynolds numbers, with and without leading edge grit roughness. In general, with leading edge grit roughness applied, the vortex generators restored 85 percent of the baseline level of maximum lift coefficient but with a more sudden stall break and at a higher angle of attack than the baseline.

14. SUBJECT TERMS

15. NUMBER OF PAGES

wind energy; horizontal-axis wind turbine; wind tunnel test data; wind turbine airfoil

16. PRICE CODE

17. SECURITY CLASSIFICATION OF REPORT

Unclassified
18. SECURITY CLASSIFICATION OF THIS PAGE Unclassified
19. SECURITY CLASSIFICATION OF ABSTRACT Unclassified
20. LIMITATION OF ABSTRACT

UL 